University of Tennessee Health Science Center

UTHSC Digital Commons

\title{
Population Pharmacokinetics of Therapeutic Monoclonal Antibodies: Examples and Estimation Method Performance Differences
}

Nathanael Le Dirks

University of Tennessee Health Science Center

Follow this and additional works at: https://dc.uthsc.edu/dissertations

Part of the Pharmacy and Pharmaceutical Sciences Commons

\section{Recommended Citation}

Dirks, Nathanael Le, "Population Pharmacokinetics of Therapeutic Monoclonal Antibodies: Examples and Estimation Method Performance Differences" (2010). Theses and Dissertations (ETD). Paper 63. http://dx.doi.org/10.21007/etd.cghs.2010.0072. 


\title{
Population Pharmacokinetics of Therapeutic Monoclonal Antibodies: Examples and Estimation Method Performance Differences
}

\begin{abstract}
Over the past two decades, there has been an increase in the research and development and therapeutic application of monoclonal antibodies (mAbs). An application of pharmacokinetic (PK) and pharmacodynamic concepts that has likely contributed to the success of the pre-clinical and clinical drug development of therapeutic mAbs is population PK, which attempts to quantify the typical disposition characteristics and sources of PK variability (such as between-subject, within-subject, and inter-occasion variability) within study populations. Population PK also attempts to identify and quantify the impact of covariates on systemic drug exposure and assess their potential implications for clinical dosing. The general theme of my dissertation research was population PK modeling of therapeutic mAbs, which focused on the population PK modeling of cetuximab, and the evaluation of different estimation methods for population PK modeling of therapeutic mAbs with nonlinear PK characteristics.
\end{abstract}

Cetuximab is a therapeutic mAb directed against the epidermal growth factor receptor and is indicated in the treatment of squamous cell carcinoma of the head and neck (SCCHN). I performed a population PK analysis of cetuximab using nonlinear mixed effects modeling and the software NONMEM. A total of 912 cetuximab concentrations were available from 143 patients with recurrent and/or metastatic SCCHN enrolled in two phase I/II studies. The PK of cetuximab was best described by a two-compartment model with Michaelis-Menten type saturable elimination. Mean population estimates (between-subject variability, \%CV) of the PK parameters were: $V_{\max } 4.38 \mathrm{mg} / \mathrm{hr}(15.4 \%), \mathrm{K}_{\mathrm{m}} 74 \mu \mathrm{g} / \mathrm{ml}, \mathrm{V}_{1} 2.83 \mathrm{~L}(18.6 \%), \mathrm{V}_{2}$ $2.43 \mathrm{~L}(56.4 \%)$, and Q $0.103 \mathrm{~L} / \mathrm{hr}(97.2 \%)$. Ideal body weight and white blood cell count were identified as predictors of $\mathrm{V}_{\text {max }}$, and total body weight as a predictor of $\mathrm{V}_{1}$. My findings suggested that clinical dose adjustments beyond the approved body surface area-based dosing of cetuximab may be warranted in patients with extreme deviations of their actual body weight from ideal body weight. Agreement between simulated and measured concentrations for up to 43 weeks of therapy indicated that the final population PK model was able to adequately describe the nonlinear PK of cetuximab in patients with SCCHN at the currently approved dosage regimen, and that the cetuximab PK parameters remained constant during prolonged therapy.

Nonlinear eliminaton is a common characteristic of the PK of therapeutic mAbs. Accordingly, PK models with nonlinear elimination are commonly used in population PK analyses of mAbs, but difficulties detecting and characterizing this nonlinear PK have been reported in a number of studies. The challenge with detecting and characterizing the nonlinear elimination of therapeutic mAbs may not only be dependent on the clinical study design, but also on the estimation method used for the population PK analysis. However, little work has been done so far evaluating population estimation methods using PK models that are representative of the typical disposition characteristics of therapeutic mAbs. In order to address this question, I conducted a simulation study to compare the parameter estimation performance of the first-order (FO), first-order conditional estimation with interaction (FOCE-I), and Laplacian estimation with interaction (LAP-I) methods in NONMEM and a Bayesian Markov Chain Monte Carlo (MCMC) method in WinBUGS when applied to population PK modeling of therapeutic mAbs with nonlinear PK. The Bayesian MCMC method was evaluated with both vague and informative priors. Published findings of population PK analyses of therapeutic mAbs were used to define the informative priors. Simulations were performed with uncertainty included simultaneously on all parameters in the population PK model in order to evaluate the sensitivity of estimation performance to uncertainty in the simulation model parameters. The impact of study design on estimation performance was explored by evaluating the methods under a dose-ranging design ('informative design') and four different single dose level designs at different dose levels ('uninformative designs'). 
Under all study designs, the FO method generally produced larger bias and lower precision for all model parameters compared to the other estimation methods. Comparison between the methods in NONMEM and WinBUGS was limited to the informative and uninformative $600 \mathrm{mg}$ dose level study designs due to prolonged run times with WinBUGS. Under the informative study design, bias and precision for all model parameters was less than $\pm 25 \%$ and $52 \%$, respectively, for FOCE-I, LAP-I, and Bayesian MCMC with both sets of priors. Under the uninformative $600 \mathrm{mg}$ dose level design, the estimation performance of FOCE-I and LAP-I decreased as bias and precision for many of the model parameters, in particular those related to nonlinear elimination, significantly increased to $\pm 40-173 \%$ and $53-173 \%$, respectively, while Bayesian MCMC with informative priors provided a clear performance advantage producing results that were comparable to those under the informative design. Under both informative and uninformative $600 \mathrm{mg}$ dose level designs, the estimation performance of FOCE-I, LAP-I, and Bayesian MCMC showed sensitivity to uncertainty in the simulation parameter values for one or more parameters. This was especially evident when informative Bayesian priors were used under the uninformative $600 \mathrm{mg}$ dose level design, which was expected given the relative uninformativeness of the data under this particular design.

The findings from this work should be of value to pharmacometricians involved in population PK modeling of therapeutic mAbs. When sufficient concentration-time data are available to characterize the nonlinear elimination of the mAb, then FOCE-I, LAP-I, or Bayesian MCMC would likely be suitable for the populatin PK analysis. In situations where insufficient data are available to characterize the nonlinear elimination of the $\mathrm{mAb}$, and relevant prior information is readily available, the use of a Bayesian MCMC method with informative priors should be considered.

\section{Document Type}

Dissertation

\section{Degree Name}

Doctor of Philosophy (PhD)

\section{Program}

Pharmaceutical Sciences

\section{Research Advisor}

Bernd Meibohm, Ph.D.

\section{Keywords}

Bayesian Markov Chain Monte Carlo, maximum likelihood, monoclonal antibody, nonlinear mixed effects modeling, population pharmacokinetics, therapeutic protein

\section{Subject Categories}

Medicine and Health Sciences | Pharmacy and Pharmaceutical Sciences 
POPULATION PHARMACOKINETICS OF THERAPEUTIC MONOCLONAL ANTIBODIES:

EXAMPLES AND ESTIMATION METHOD PERFORMANCE DIFFERENCES

\author{
A Dissertation \\ Presented for \\ The Graduate Studies Council \\ The University of Tennessee \\ Health Science Center
}

\begin{abstract}
In Partial Fulfillment
Of the Requirements for the Degree

Doctor of Philosophy

From The University of Tennessee
\end{abstract}

By

Nathanael Le Dirks

May 2010 
Chapter 1 (C) 2010 by Adis Data Information BV Chapter 3 (C) 2008 by American College of Clinical Pharmacology All other material $\odot 2010$ by Nathanael L. Dirks 
To my parents and brothers for their endless love and support and

In loving memory of my grandparents, Frank and Lovina Timmerman 


\section{ACKNOWLEDGMENTS}

The daunting task of obtaining my Pharm.D. and Ph.D. could not have come to fruition without the tremendous support and encouragement from so many people. First and foremost, I would like to express my deepest gratitude and sincere appreciation to my amazing research advisor, Dr. Bernd Meibohm, for being my biggest advocate throughout this endevour and for instilling in me the qualities of being a great and respected scientist. The other members of my graduate committee, Dr. Marc Gastonguay, Dr. Duane D. Miller, Dr. John C. Panetta, and Dr. Charles R. Yates, were also an integral part of my graduate education and research work, and I would like to thank them for their invaluable suggestions and guidance. I owe a special thanks to Dr. Gastonguay for giving me the incredible opportunity to carry out a large part of my research work at Metrum Research Group. I would like to express my appreciation to everyone at Metrum, especially Dr. William R. Gillespie, Dr. Matthew M. Riggs, and Dr. William Knebel, for all of their input and assistance with the portion of my research work related to the comparative performance of different estimation methods for population pharmacokinetic modeling of therapeutic monoclonal antibodies. I would also like to thank Dr. Arno Nolting and Dr. Andreas Kovar from Merck KGaA for supplying the clinical trial data for my population pharmacokinetic analysis of cetuximab.

Words cannot describe how grateful and thankful I am for all of the love and support from my parents, Randy and Rebecca, and my two brothers, Joshua and Andrew. I am truly blessed to have such an amazing family, and the completion of my Pharm.D./Ph.D. would not have been possible without them by my side.

Many thanks to all of my friends and fellow graduate students (both past and present), Dr. Nageshwar Budha, Dr. Margaret Thomson, Yi Zhang, Pavan Vaddady, Wararat Limothai, Dr. Satyendra Suryawanshi, Sonia Bedi, Dr. Himanshu Bhattacharjee, Dr. Manish Gupta, Dr. Shen Li, Fei Ma, Dr. Nitin Mehrotra, Chaela Presley, Dr. Pengfei Song, Dr. Lisa Tang, Karin Thompson, and Dr. Wenhui Zhang, for all of their friendship and support. A special thanks to my best friends, Drs. Les and Melissa Stuart, for making my stay in Memphis a memorable and enjoyable one.

I would like to express my gratitude to Dr. Ken and Sherry Nuss from the University of Northern Iowa for their friendship and mentorship during my undergraduate studies, and for always believing that I would go on to do great things. Much of my impetus to pursue a Ph.D. in pharmaceutical sciences was the result a summer internship during my final year of college with Drs. Timothy Ryken and Zita Sibenaller in the Department of Neurosurgery at the University of Iowa, and I would like to thank them both for giving me such an incredible and educational opportunity.

I would like thank the American Foundation for Pharmaceutical Education, the American Association for Pharmaceutical Scientists, and GlaxoSmithKline, for their financial support of my pre-doctoral fellowship through. Finally, I would like to thank God for all of his blessings and for watching over me. 


\begin{abstract}
Over the past two decades, there has been an increase in the research and development and therapeutic application of monoclonal antibodies (mAbs). An application of pharmacokinetic (PK) and pharmacodynamic concepts that has likely contributed to the success of the pre-clinical and clinical drug development of therapeutic $\mathrm{mAbs}$ is population $\mathrm{PK}$, which attempts to quantify the typical disposition characteristics and sources of PK variability (such as between-subject, within-subject, and inter-occasion variability) within study populations. Population PK also attempts to identify and quantify the impact of covariates on systemic drug exposure and assess their potential implications for clinical dosing. The general theme of my dissertation research was population PK modeling of therapeutic mAbs, which focused on the population PK modeling of cetuximab, and the evaluation of different estimation methods for population PK modeling of therapeutic mAbs with nonlinear PK characteristics.

Cetuximab is a therapeutic mAb directed against the epidermal growth factor receptor and is indicated in the treatment of squamous cell carcinoma of the head and neck (SCCHN). I performed a population PK analysis of cetuximab using nonlinear mixed effects modeling and the software NONMEM. A total of 912 cetuximab concentrations were available from 143 patients with recurrent and/or metastatic SCCHN enrolled in two phase I/II studies. The PK of cetuximab was best described by a twocompartment model with Michaelis-Menten type saturable elimination. Mean population estimates (between-subject variability, $\% \mathrm{CV}$ ) of the PK parameters were: $\mathrm{V}_{\max } 4.38$ $\mathrm{mg} / \mathrm{hr}(15.4 \%), \mathrm{K}_{\mathrm{m}} 74 \mu \mathrm{g} / \mathrm{ml}, \mathrm{V}_{1} 2.83 \mathrm{~L}(18.6 \%), \mathrm{V}_{2} 2.43 \mathrm{~L}(56.4 \%)$, and Q $0.103 \mathrm{~L} / \mathrm{hr}$ $(97.2 \%)$. Ideal body weight and white blood cell count were identified as predictors of $\mathrm{V}_{\max }$, and total body weight as a predictor of $\mathrm{V}_{1}$. My findings suggested that clinical dose adjustments beyond the approved body surface area-based dosing of cetuximab may be warranted in patients with extreme deviations of their actual body weight from ideal body weight. Agreement between simulated and measured concentrations for up to 43 weeks of therapy indicated that the final population PK model was able to adequately describe the nonlinear PK of cetuximab in patients with SCCHN at the currently approved dosage regimen, and that the cetuximab PK parameters remained constant during prolonged therapy.
\end{abstract}

Nonlinear eliminaton is a common characteristic of the PK of therapeutic mAbs. Accordingly, PK models with nonlinear elimination are commonly used in population PK analyses of $\mathrm{mAbs}$, but difficulties detecting and characterizing this nonlinear PK have been reported in a number of studies. The challenge with detecting and characterizing the nonlinear elimination of therapeutic mAbs may not only be dependent on the clinical study design, but also on the estimation method used for the population PK analysis. However, little work has been done so far evaluating population estimation methods using PK models that are representative of the typical disposition characteristics of therapeutic mAbs. In order to address this question, I conducted a simulation study to compare the parameter estimation performance of the first-order (FO), first-order conditional estimation with interaction (FOCE-I), and Laplacian estimation with 
interaction (LAP-I) methods in NONMEM and a Bayesian Markov Chain Monte Carlo (MCMC) method in WinBUGS when applied to population PK modeling of therapeutic mAbs with nonlinear PK. The Bayesian MCMC method was evaluated with both vague and informative priors. Published findings of population PK analyses of therapeutic $\mathrm{mAbs}$ were used to define the informative priors. Simulations were performed with uncertainty included simultaneously on all parameters in the population PK model in order to evaluate the sensitivity of estimation performance to uncertainty in the simulation model parameters. The impact of study design on estimation performance was explored by evaluating the methods under a dose-ranging design ('informative design') and four different single dose level designs at different dose levels ('uninformative designs').

Under all study designs, the FO method generally produced larger bias and lower precision for all model parameters compared to the other estimation methods. Comparison between the methods in NONMEM and WinBUGS was limited to the informative and uninformative $600 \mathrm{mg}$ dose level study designs due to prolonged run times with WinBUGS. Under the informative study design, bias and precision for all model parameters was less than $\pm 25 \%$ and $52 \%$, respectively, for FOCE-I, LAP-I, and Bayesian MCMC with both sets of priors. Under the uninformative $600 \mathrm{mg}$ dose level design, the estimation performance of FOCE-I and LAP-I decreased as bias and precision for many of the model parameters, in particular those related to nonlinear elimination, significantly increased to $\pm 40-173 \%$ and $53-173 \%$, respectively, while Bayesian MCMC with informative priors provided a clear performance advantage producing results that were comparable to those under the informative design. Under both informative and uninformative $600 \mathrm{mg}$ dose level designs, the estimation performance of FOCE-I, LAP-I, and Bayesian MCMC showed sensitivity to uncertainty in the simulation parameter values for one or more parameters. This was especially evident when informative Bayesian priors were used under the uninformative $600 \mathrm{mg}$ dose level design, which was expected given the relative uninformativeness of the data under this particular design.

The findings from this work should be of value to pharmacometricians involved in population PK modeling of therapeutic mAbs. When sufficient concentration-time data are available to characterize the nonlinear elimination of the mAb, then FOCE-I, LAP-I, or Bayesian MCMC would likely be suitable for the populatin PK analysis. In situations where insufficient data are available to characterize the nonlinear elimination of the $\mathrm{mAb}$, and relevant prior information is readily available, the use of a Bayesian MCMC method with informative priors should be considered. 


\section{TABLE OF CONTENTS}

\begin{tabular}{|c|}
\hline 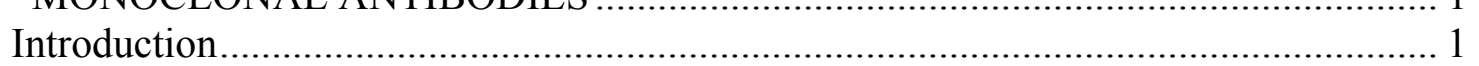 \\
\hline Antibody Structure \\
\hline 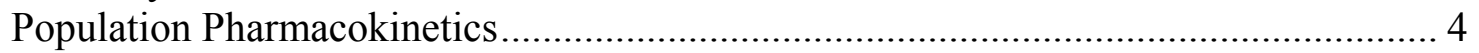 \\
\hline Antibody Absorption \\
\hline Antibody Distribution \\
\hline Antibody Clearance \\
\hline 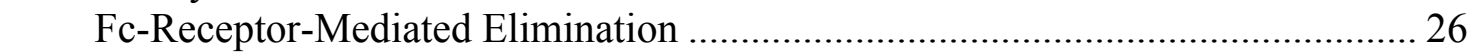 \\
\hline 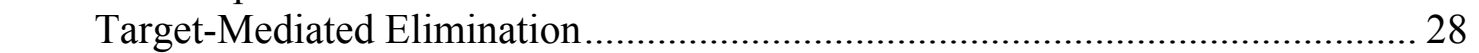 \\
\hline 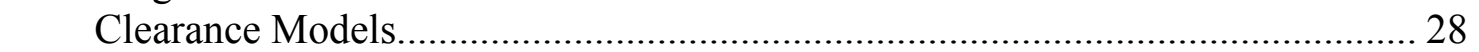 \\
\hline 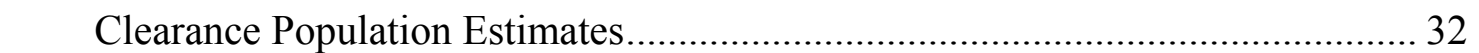 \\
\hline 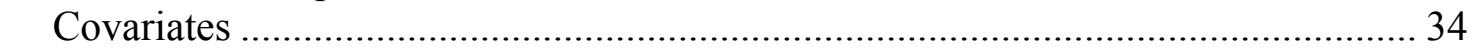 \\
\hline 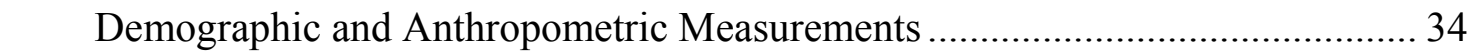 \\
\hline 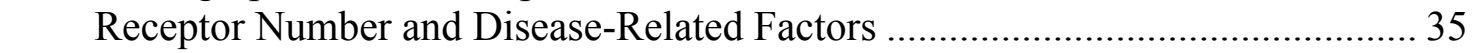 \\
\hline 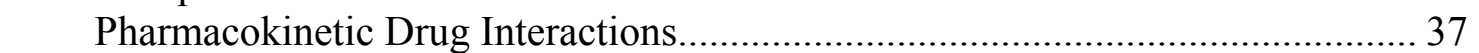 \\
\hline 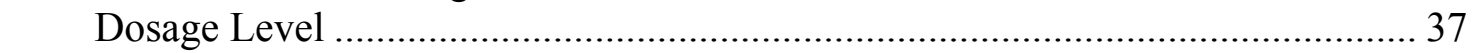 \\
\hline Immunogenicity \\
\hline 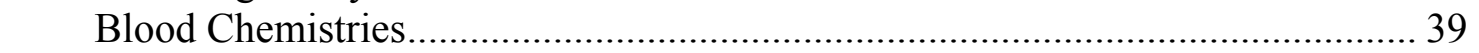 \\
\hline 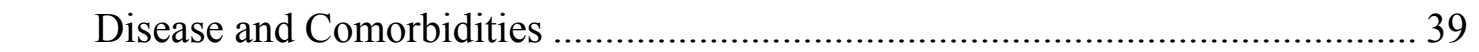 \\
\hline 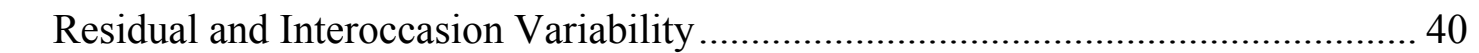 \\
\hline Conclusion \\
\hline
\end{tabular}

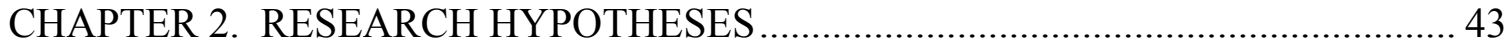

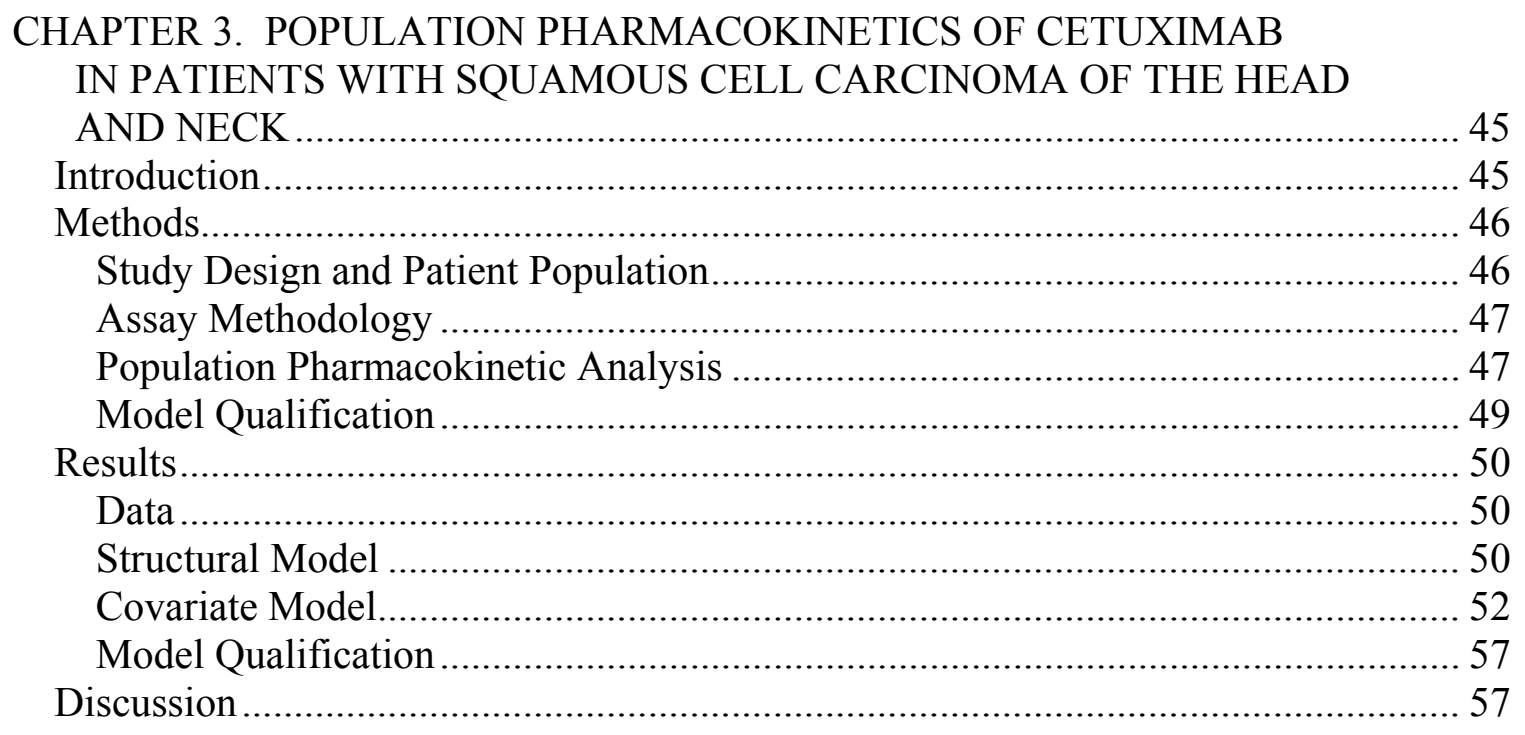


CHAPTER 4. COMPARATIVE PERFORMANCE OF BAYESIAN MARKOV CHAIN MONTE CARLO AND MAXIMUM LIKELIHOOD POPULATION ESTIMATION METHODS WHEN APPLIED TO NONLINEAR PHARMACOKINETICS OF MONOCLONAL ANTIBODIES 65

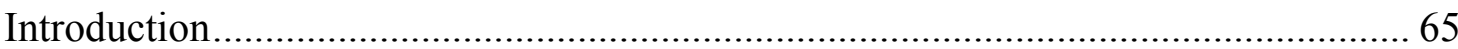

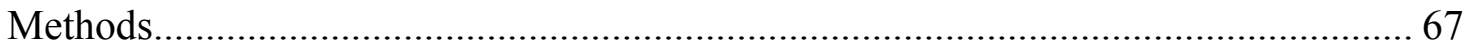

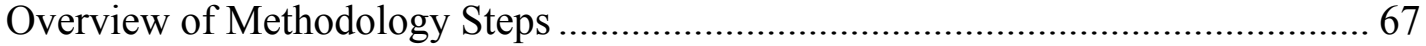

Population Pharmacokinetic Model ......................................................................... 67

Simulation Parameter Uncertainty Distributions ................................................ 70

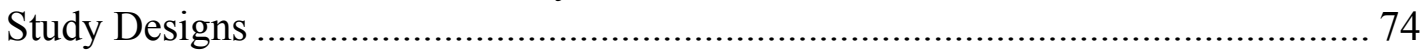

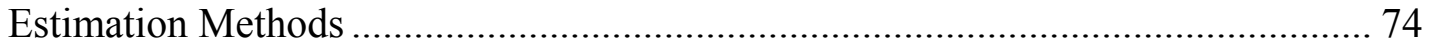

Evaluation of Estimation Performance …............................................................ 80

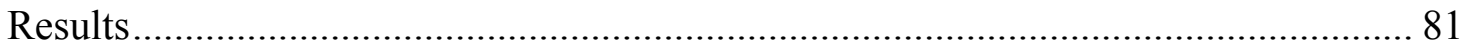

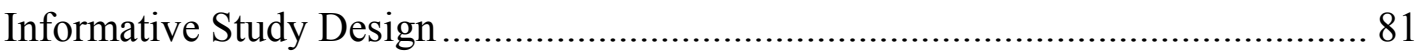

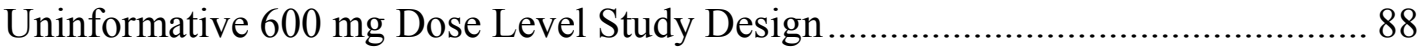

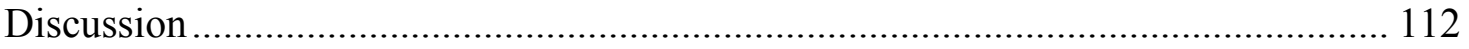

CHAPTER 5. SUMMARY OF DISSERTATION RESEARCH …............................ 124

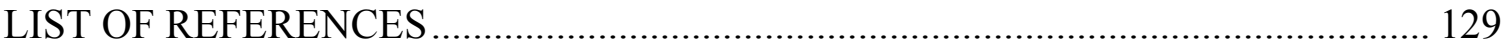

APPENDIX A: NONMEM CONTROL STREAM AND OUTPUT FOR THE FINAL CETUXIMAB POPULATION PK MODEL ............................................... 141

APPENDIX B: EXAMPLE COMPUTER CODES USED FOR THE SIMULATION AND ESTIMATION STEPS IN CHAPTER 4............................. 150

APPENDIX C: CHAPTER 4 SUPPLEMENTAL FIGURES AND TABLES .............. 181

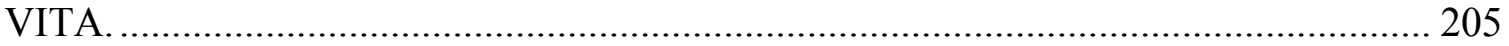




\section{LIST OF TABLES}

Table 1-1. Therapeutic monoclonal antibodies with published population pharmacokinetic analyses

Table 1-2. Population estimates and their between-subject variability

[\%RSE or confidence interval] of absorption related parameters.

Table 1-3. Population estimates and their between-subject variability [\%RSE or confidence interval] of distribution related parameters 8

Table 1-4. Population estimates and their between-subject variability [\%RSE or confidence interval] of clearance related parameters

Table 1-5. Identified covariates and their estimated effects on pharmacokinetic parameters $[\%$ RSE or confidence interval] ....................................... 16

Table 1-6. Residual variability estimates [\%RSE or confidence interval] $\quad$................ 21

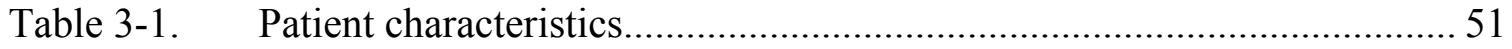

Table 3-2. Final population pharmacokinetic model and its parameter estimates with bootstrap-derived $90 \%$ confidence intervals.

Table 4-1. Bias (median percent estimation error) in the population pharmacokinetic model parameters under the informative study design.

Table 4-2. Precision (median absolute percent estimation error) in the population pharmacokinetic model parameters under the informative study design.

Table 4-3. Bias (median percent estimation error) in the population pharmacokinetic model parameters under the uninformative $600 \mathrm{mg}$ dose level study design.

Table 4-4. Precision (median absolute percent estimation error) in the population pharmacokinetic model parameters under the uninformative $600 \mathrm{mg}$ dose level study design.

Table C-1. Bias (median percent estimation error) in the population pharmacokinetic model parameters under the uninformative 200, 500, and $800 \mathrm{mg}$ dose level study designs. 
Table C-2. Precision (median absolute percent estimation error) in the population pharmacokinetic model parameters under the uninformative 200, 500, and $800 \mathrm{mg}$ dose level study designs. 


\section{LIST OF FIGURES}

Figure 1-1. Linear representation of the structure of immunoglobulin G (A) and the different classes of therapeutic monoclonal antibodies (B).................. 3

Figure 1-2. Pharmacokinetic behavior of a monoclonal antibody with parallel linear and nonlinear elimination pathways.

Figure 3-1. Goodness-of-fit plots for the final model 54

Figure 3-2. Cetuximab serum concentration-time profile in six representative patients (patient ID indicated) over a period of 28 days after initiation of therapy with a dose of $400 \mathrm{mg} / \mathrm{m}^{2}$, followed by weekly doses of $250 \mathrm{mg} / \mathrm{m}^{2}$. 56

Figure 3-3. Results of the predictive check for peak and trough concentrations at week 4 of therapy. 58

Figure 3-4. Scatterplot of the results from the predictive check of peak and trough concentrations at week 4 of therapy in the model building dataset.

Figure 3-5. Results of the simulation of concentrations in the model evaluation dataset beyond week 8 of therapy.

Figure 4-1. Overview of the methodology of the simulation and estimation steps.

Figure 4-2. Boxplots of percent estimation errors for the population pharmacokinetic model parameters under the informative study design.

Figure 4-3. Global sensitivity analysis results from the informative study design.

Figure 4-4. Simulated prediction intervals for concentrations during week 4 of therapy based on the true parameter values and biased parameter values derived from the FOCE-I results under the informative study design.

Figure 4-5. Simulated prediction intervals for concentrations during week 4 of therapy based on the true parameter values and biased parameter values derived from the Bayesian MCMC (with uninformative priors) results under the informative study design. 
Figure 4-6. Boxplots of percent estimation errors for the population pharmacokinetic model parameters under the uninformative $600 \mathrm{mg}$ dose level study design.

Figure 4-7. Global sensitivity analysis results from the uninformative $600 \mathrm{mg}$ dose level study design.

Figure 4-8. Simulated prediction intervals for concentrations during week 4 of therapy based on the true parameter values and biased parameter values derived from the FOCE-I results under the uninformative $600 \mathrm{mg}$ dose level study design.

Figure 4-9. $\quad$ Simulated prediction intervals for concentrations during week 4 of therapy based on the true parameter values and biased parameter values derived from the LAP-I results under the uninformative $600 \mathrm{mg}$ dose level study design.

Figure 4-10. Simulated prediction intervals for concentrations during week 4 of therapy based on the true parameter values and biased parameter values derived from the Bayesian MCMC (with informative priors) results under the uninformative $600 \mathrm{mg}$ dose level study design.

Figure 4-11. Probability density plots of the parameter uncertainty distributions used for simulation of the trial replicates.

Figure 4-12. Scatterplot matrix of parameter values drawn from the multivariate lognormal uncertainty distribution defined for the parameters CLL, V1, V2, and Q, for simulating the trial replicates.

Figure 4-13. Plot of clearance versus dose based on a noncompartmental analysis of a simulated replicate dataset under the informative study design.

Figure C-1. Markov Chain Monte Carlo chain histories (first set of plots), probability densities of the posterior distributions (second set of plots), and Gelman-Rubin-Brooks plots (third set of plots) for a representative estimation run using Bayesian informative priors under the informative study design.

Figure C-2. Markov Chain Monte Carlo chain histories (first set of plots), probability densities of the posterior distributions (second set of plots), and Gelman-Rubin-Brooks plots (third set of plots) for a representative estimation run using Bayesian uninformative priors under the informative study design. 
Figure C-3. Markov Chain Monte Carlo chain histories (first set of plots), probability densities of the posterior distributions (second set of plots), and Gelman-Rubin-Brooks plots (third set of plots) for a representative estimation run using Bayesian informative priors under the uninformative $600 \mathrm{mg}$ study design. 


\section{LIST OF ABBREVIATIONS}

5-FU

ADCC

AIC

BSA

CDC

CDR

$\mathrm{CL} / \mathrm{F}$

$\mathrm{CL}_{\text {int }}$

$\mathrm{CL}_{\mathrm{L}}$

$\mathrm{CL}_{\mathrm{NL}}$

$\mathrm{CL}_{\mathrm{T}}$

EGFR

$\mathrm{F}$

Fab

$\mathrm{Fc}$

FcRn

FDA

FO

FOCE

FOCE-I

IBW

$\operatorname{IgG}$

IM

IOV

IV

$\mathrm{K}_{12}$

$\mathrm{K}_{21}$

$\mathrm{Ka}$

$\mathrm{Kd}$

$\mathrm{K}_{\mathrm{m}}$

LAP

LAP-I

$\mathrm{mAb}$

MCMC

MAPE

MPE

OFV

$\triangle \mathrm{OFV}$

PD

PK

Q

$\mathrm{SC}$

SCCHN 5-fluorouracil

Antibody-dependent cellular cytotoxicity Akaike information criterion

Body surface area Complement-dependent cytotoxicity Complementarity determining region

Apparent clearance

Intrinsic clearance

Clearance of linear elimination pathway Clearance of nonlinear elimination pathway

Total clearance

Epidermal growth factor receptor

Absolute bioavailability Antigen binding fragment

Crystallizable fragment

Neonatal Fc receptor Food and Drug Administration

First-order

First-order conditional estimation First-order conditional estimation with interaction Ideal body weight Immunoglobulin $\mathrm{G}$

Intramuscular

Interoccasion variability

Intravenous

Distribution rate constant from central to peripheral compartment Distribution rate constant from peripheral to central compartment

Absorption rate constant

Dissociation constant

Concentration at half-maximum elimination rate Laplacian

Laplacian estimation with interaction

Monoclonal antibody

Markov Chain Monte Carlo

Median absolute percent estimation error

Median percent estimation error

Objective function value

Change in objective function value

Pharmacodynamics

Pharmacokinetics

Intercompartmental clearance

Subcutaneous

Squamous cell carcinoma of the head and neck 
$\mathrm{V}_{1}$

$\mathrm{V}_{2}$

$\mathrm{V} / \mathrm{F}$

$\mathrm{V}_{\max }$

$\mathrm{V}_{\mathrm{ss}}$

WBC

WGT
Central compartment volume Peripheral compartment volume Apparent volume of distribution Maximum elimination rate Volume of distribution at steady-state White blood cell

Body weight 


\section{CHAPTER 1. POPULATION PHARMACOKINETICS OF THERAPEUTIC MONOCLONAL ANTIBODIES*}

\section{Introduction}

Over the past two decades there has been an increase in the research and development and therapeutic application of monoclonal antibodies (mAbs). Currently, there are over $20 \mathrm{mAb}$ products (i.e. antibodies, antibody fragments, or antibodyconjugates) approved by the U.S. Food and Drug Administration (FDA) for therapeutic use in areas such as oncology, immunology, ophthalmology, cardiovascular disease, and infectious disease. As most approved therapeutic mAb products are intact and unconjugated, this review will focus on this class.

It is estimated that half of all current projects in new drug development are biologics, with mAbs being the predominant class of biologics under clinical study (1). In 2008, it was reported that there were over 200 mAbs undergoing clinical study (2). One likely reason for the continued success of the clinical development of mAbs is the application and integration of pharmacokinetic (PK) and pharmacodynamic (PD) concepts in all stages of pre-clinical and clinical drug development $(3,4)$. Such implementation of PK/PD modeling and simulation in drug product development provides a rational, scientifically based framework for efficient decision making regarding the selection of potential drug candidates, for maximum information gain from the performed experiments and studies, and for conducting fewer, more focused clinical trials with improved efficiency and cost effectiveness (5). One application of PK/PD concepts in drug development is population PK, which attempts to quantify the typical disposition characteristics and sources of PK variability (such as between-subject, withinsubject, and inter-occasion) within study populations. Population PK also attempts to identify and quantify the impact of covariates on systemic drug exposure, and assess their potential implications for clinical dosing. Findings from population PK analyses are included in the drug labeling of over half of the approved therapeutic mAb products, and several $\mathrm{mAb}$ population $\mathrm{PK}$ studies have also been published in the scientific literature. The aim of this report is to review the population PK of therapeutic intact mAbs, while highlighting important similarities/dissimilarities across different mAbs with respect to their drug disposition characteristics. The report will be supplemented with discussions on the general PK behavior of mAbs and their structural properties, which have partially been reviewed elsewhere in different context (6-9).

\section{Antibody Structure}

The basic structure of human antibodies or immunoglobulins (Ig) consists of two

*This chapter adapted with permission. Dirks NL, Meibohm B. Population pharmacokinetics of therapeutic monoclonal antibodies. Clin Pharmacokinet 2010;49(10) In press. 
identical heavy chains and two identical light chains joined together by a number of disulfide bridges. There are five classes (isotypes) of antibodies termed IgG, IgA, IgM, $\operatorname{IgE}$, and $\operatorname{IgD}$, which share the same basic structure, but differ with regards to their heavy chains $\gamma, \alpha, \mu, \varepsilon, \delta$, respectively (10). In addition to the different heavy chain structures, there are also two types of light chains ( $\kappa$ and $\lambda$ ). IgG is the most predominant of the five isotypes with a serum concentration of approximately $12 \mathrm{mg} / \mathrm{ml}$, representing about $70-80 \%$ of total serum Ig (10). The IgG class can be further subdivided into four subclasses (or subisotypes), IgG1, IgG2, IgG3, and IgG4, based on structural differences in the $\gamma$ heavy chain. This review focuses on IgG, as all of the population PK analyses included in the discussion were of therapeutic mAbs of the IgG type. All of the approved therapeutic intact $\mathrm{mAbs}$ are also of the IgG isotype.

The structure of $\operatorname{IgG}$ is depicted in Figure 1-1A. The heavy and light chains of IgG are made up of variable and constant domains based on sequence similarities between different IgG molecules. The IgG molecule is comprised of three basic units: two identical antigen binding fragments (Fabs) and the crystallizable fragment $(\mathrm{Fc})$. The variable heavy, constant heavy 1, variable light, and constant light domains make up the $\mathrm{Fab}$, and the $\mathrm{Fc}$ is made up of the constant heavy 2 and constant heavy 3 domains. The whole $\operatorname{IgG}$ molecule has a molecular weight of $150 \mathrm{kDa}$, and intact mAbs of the same isotype have an approximately similar molecular weight. Within the variable regions of both the heavy chain and light chain, there are three sections called complementarity determining regions (CDRs). These hypervariable regions are associated with the mAb's affinity and specificity for a given antigen. The structural diversity of the Fab allows different IgG molecules to recognize a wide variety of antigens, while the ability to trigger immune effector functions through interaction with Fc $\gamma$ receptors and complement, such as antibody-dependent cellular cytotoxicity (ADCC) and complementdependent cytotoxicity (CDC), are conferred upon by consistency in the Fc structure. The $\mathrm{Fc}$ region of the $\mathrm{mAb}$ also interacts with another important $\mathrm{Fc}$ receptor for $\mathrm{IgG}$, the neonatal $\mathrm{Fc}$ receptor $(\mathrm{FcRn})$, which acts in part as a salvage receptor protecting $\mathrm{IgG}$ from intracellular catabolism. As will become evident later in the review, the Fab and Fc regions are not only important to antibody function, but can significantly influence the PK behavior of mAbs.

Muromonab-CD3 (Orthoclone OKT3), a murine IgG2a antibody against CD3 indicated for the treatment of acute organ rejection, became the first $\mathrm{mAb}$ approved for therapeutic use in 1986. However, the use of mAbs of murine origin in the clinic has been limited due to several factors including their short elimination half-lives and high immunogenic potential. To overcome these limitations with murine mAbs, advances in biotechnology such as recombinant engineering have facilitated the development of chimeric, humanized, and fully human antibodies, which contain a larger content of human protein sequence (11). These three structural classes of mAbs are depicted in Figure 1-1B. In chimeric mAbs, the antibody is constructed by transferring murine variable regions to constant regions of human origin. This reduces the murine content in chimeric mAbs to about one-third. In humanized or CDR-grafted mAbs, the CDRs (and possibly parts of the framework regions surrounding the CDRs) are of murine origin, which makes up about $5-10 \%$ of the $\mathrm{mAb}$. Although the immunogenic potential of a 


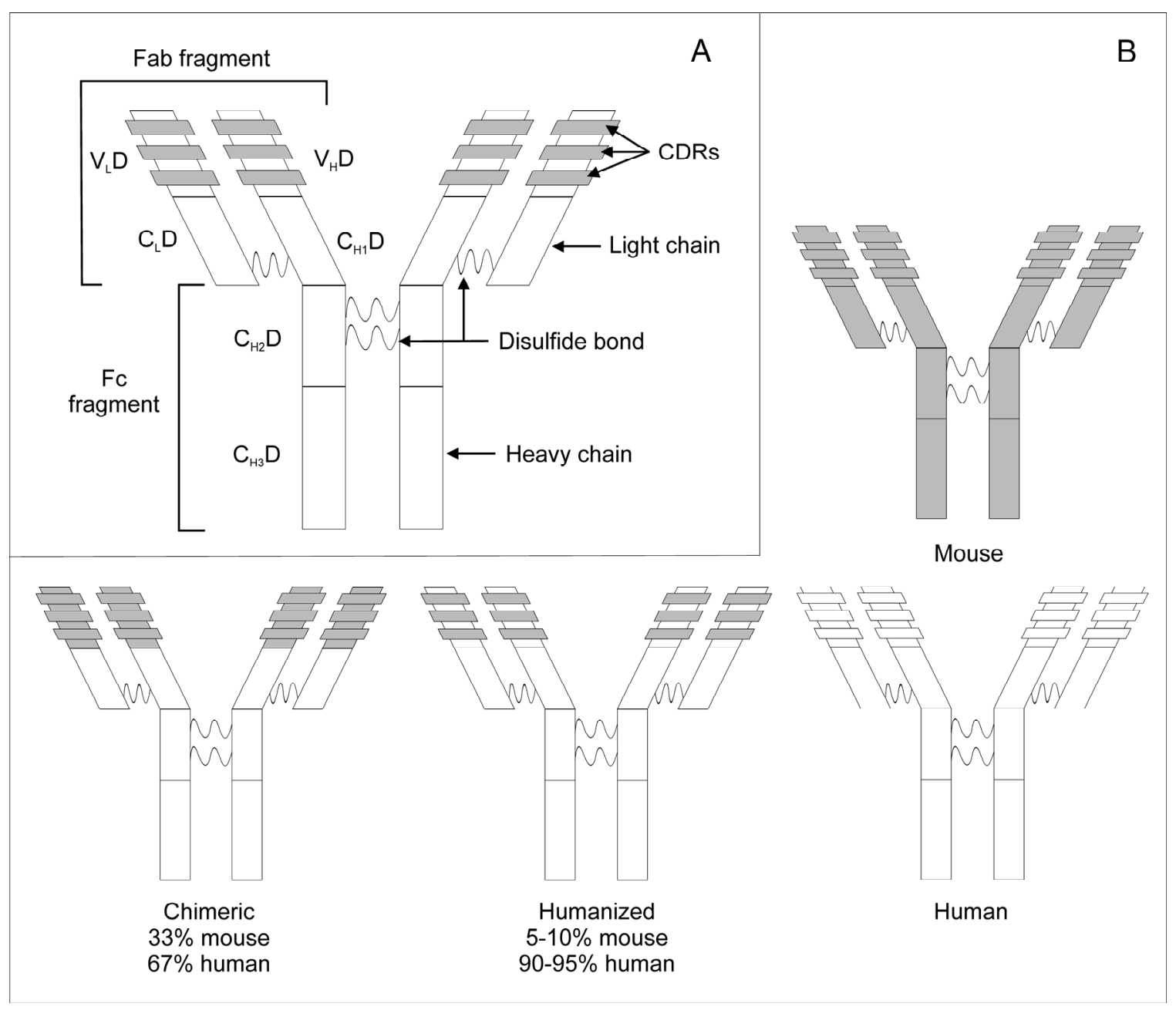

Figure 1-1. Linear representation of the structure of immunoglobulin $G(A)$ and the different classes of therapeutic monoclonal antibodies (B). $\mathbf{C D R}=$ complementarity determining region; $\mathbf{C}_{\mathbf{H} 1} \mathrm{D}, \mathbf{C}_{\mathbf{H} 2} \mathrm{D}$, and $\mathbf{C}_{\mathbf{H} 3} \mathrm{D}=$ constant heavy chain domains; $\mathbf{C}_{\mathbf{L}} \mathbf{D}=$ constant light chain domain; Fab $=$ antigen binding fragment; $\mathbf{F c}=$ crystallizable fragment; $\mathbf{V}_{\mathbf{H}} \mathbf{D}=$ variable heavy chain domain; $\mathbf{V}_{\mathbf{L}} \mathbf{D}=$ variable light chain domain. 
$\mathrm{mAb}$ is expected to decrease with decreasing murine content (murine $>$ chimeric $>$ humanized $>$ human), all classes of therapeutic mAbs have the potential to trigger the formation of human antibodies against the mAb (6). Such anti$\mathrm{mAb}$ antibodies can have a significant effect on both the PK and PD of the therapeutic $\mathrm{mAb}(7,9)$. As will be discussed later in the review, the PK effect of the presence of antibodies to the therapeutic mAb can be assessed by including this as a covariate in the population model. Currently, most approved therapeutic mAbs are of the humanized type. Fully human mAbs that have been approved by the FDA include adalimumab, golimumab, panitumumab, and ustekinumab.

\section{Population Pharmacokinetics}

While a number of concepts related to population analysis are mentioned in this review (e.g., between-subject variability and residual variability), a discussion of the basic concepts and principles of population PK modeling is beyond the scope of this report. For readers that are not familiar with population PK modeling, there are a number of papers and books in the literature that provide an excellent review of the topic (12-16). Results from the population PK analyses of therapeutic mAbs are summarized and split into Tables 1-1 through 1-6. In some cases a population PK/PD analysis was performed, and these are noted in Table 1-1. The only non-therapeutic mAb included in the tables is $\mathrm{HuCC} 49 \Delta \mathrm{CH} 2$, which was investigated in patients undergoing radioimmunoguided surgery (17). The structural characteristics and pharmacologic target of the different mAbs are listed in Table 1-1, as well as the patient population the study was conducted in, and the model used to describe the PK of the mAb. Population parameter estimates related to $\mathrm{mAb}$ absorption, distribution, elimination, and identified covariate effects are presented in Tables 1-2 through 1-6. Of note is the fact that all of the population PK analyses were of chimeric, humanized, or fully human antibodies, except for the murine antibodies inolimomab and mabF19 (Table 1-1). Differences in the population PK of murine $\mathrm{mAbs}$ and the other mAb types were observed and will be discussed later in the review. While the patient populations in the studies cover a wide variety of clinical indications, all of the studies were conducted in adult patients. A few population PK analyses of mAbs have been conducted in pediatric patients including daclizumab (18) and SB 209763 (19), but these studies were not included in this review for comparative purposes. In the majority of the population PK studies, the nonlinear mixed effects modeling was performed using NONMEM (Icon Development Solutions, Ellicott City, MD) and the first-order conditional estimation method (FOCE) with or without the $\eta-\varepsilon$ interaction option (20). Other software and methods used in a couple of studies $(21,22)$ included a conditional first-order method implemented in Winnonmix (Pharsight corporation, Mountain View, NC), and the Monte Carlo parametric expectation maximization (MCPEM) method implemented in an augmented version of ADAPT II (Biomedical Simulations Resource, University of Southern California, Los Angeles, CA). 
Table 1-1. Therapeutic monoclonal antibodies with published population pharmacokinetic analyses.

\begin{tabular}{|c|c|c|c|c|}
\hline Antibody & Structure/Isotype & Target & Patient population & PK model \\
\hline Alemtuzumab $(23)^{\dagger}$ & CDR-grafted rat/human IgG1 & CD52 & B-cell chronic lymphocytic leukaemia & $2 \mathrm{C} \mathrm{NL}$ \\
\hline AMG 162 (denosumab) $(24)^{\dagger}$ & Human IgG2 & RANKL & Healthy postmenopausal women & $2 \mathrm{C} \mathrm{NL}+\mathrm{L}$ \\
\hline ATM-027 $(25)^{\dagger}$ & Humanized IgG1 & $\mathrm{V} \beta$ 5.2/5.3 TCR & Multiple sclerosis & $2 \mathrm{C} \mathrm{L}$ \\
\hline Basiliximab (26) & Chimeric mouse/human IgG1 & CD25 & Renal transplant & $2 \mathrm{C} \mathrm{L}$ \\
\hline Basiliximab (27) & Chimeric mouse/human IgG1 & CD25 & Liver transplant & $2 \mathrm{C} \mathrm{L}$ \\
\hline Bevacizumab (28) & CDR-grafted mouse/human IgG1 & VEGF & $\begin{array}{l}\text { Various solid tumors and colorectal, } \\
\text { non-small cell lung, and breast cancer }\end{array}$ & $2 \mathrm{C} \mathrm{L}$ \\
\hline Cetuximab (29) & Chimeric mouse/human IgG1 & EGFR & Head and neck cancer & $2 \mathrm{C} \mathrm{NL}$ \\
\hline Clenoliximab $(30)^{\dagger}$ & Chimeric macaque/human IgG4 & CD4 & Rheumatoid arthritis & $2 \mathrm{C} \mathrm{NL}$ \\
\hline CP-751, $871(31)$ & Human IgG2 & IGF-IR & Multiple myeloma and solid tumors & $2 \mathrm{C} \mathrm{NL}+\mathrm{L}^{*}$ \\
\hline Denosumab $(32)^{\dagger}$ & Human IgG2 & RANKL & $\begin{array}{l}\text { Healthy postmenopausal women and } \\
\text { breast cancer }\end{array}$ & $2 \mathrm{C} \mathrm{NL}+\mathrm{L}$ \\
\hline Efalizumab $(21)^{\dagger}$ & CDR-grafted mouse/human IgG1 & CD11a & Psoriasis & $2 \mathrm{C} \mathrm{NL}+\mathrm{L}$ \\
\hline Efalizumab (33) & CDR-grafted mouse/human IgG1 & CD11a & Psoriasis & $1 \mathrm{C} \mathrm{L}$ \\
\hline Golimumab (34) & Human IgG1 & TNF- $\alpha$ & Rheumatoid arthritis & $2 \mathrm{C} \mathrm{L}$ \\
\hline hu1 124 (efalizumab) $(35)^{\dagger}$ & CDR-grafted mouse/human IgG1 & CD11a & Psoriasis & $\begin{array}{l}2 \mathrm{C} \mathrm{NL}+\mathrm{L} \\
\mathrm{RMC}\end{array}$ \\
\hline $\mathrm{HuCC} 49 \Delta \mathrm{CH} 2$ (17) & $\begin{array}{l}\text { CDR-grafted mouse/human } \\
\text { IgG1-CH2 domain deletion }\end{array}$ & TAG-72 & Colorectal cancer & $2 \mathrm{C} \mathrm{L}$ \\
\hline Infliximab (36) & Chimeric mouse/human IgG1 & TNF- $\alpha$ & Ankylosing spondylitis & $2 \mathrm{C} \mathrm{L}$ \\
\hline Infliximab (37) & Chimeric mouse/human IgG1 & TNF- $\alpha$ & Ulcerative colitis & $2 \mathrm{C} \mathrm{L}$ \\
\hline Inolimomab $(38)^{\dagger}$ & Mouse IgG1 & CD25 & Acute graft-versus-host disease & $2 \mathrm{C} \mathrm{L}$ \\
\hline mAbF19 (39) & Mouse IgG & FAP & $\begin{array}{l}\text { Colorectal cancer and soft tissue } \\
\text { sarcoma }\end{array}$ & $2 \mathrm{C} \mathrm{L}$ \\
\hline Matuzumab (40) & CDR-grafted mouse/human IgG1 & EGFR & $\begin{array}{l}\text { Pancreatic and various cancers } \\
\text { (primarily colorectal) }\end{array}$ & $2 \mathrm{C} \mathrm{NL}+\mathrm{L}$ \\
\hline
\end{tabular}


Table 1-1 continued.

\begin{tabular}{|c|c|c|c|c|}
\hline Antibody & Structure/Isotype & Target & Patient population & PK model \\
\hline 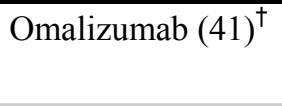 & CDR-grafted mouse/human IgG1 & $\operatorname{IgE}$ & $\begin{array}{l}\text { Asthma, allergic rhinitis, and healthy } \\
\text { atopic subjects }\end{array}$ & TMDD \\
\hline Panitumumab (42) & Human IgG2 & EGFR & $\begin{array}{l}\text { Various solid tumors and colorectal, } \\
\text { non-small cell lung, and renal cancer }\end{array}$ & $2 \mathrm{C} \mathrm{NL}+\mathrm{L}$ \\
\hline Pertuzumab (43) & CDR-grafted mouse/human IgG1 & HER2 & $\begin{array}{l}\text { Breast cancer, ovarian cancer, and } \\
\text { solid malignancies }\end{array}$ & $2 \mathrm{C} \mathrm{L}$ \\
\hline Rituximab (44) & Chimeric mouse/human IgG1 & $\mathrm{CD} 20$ & Rheumatoid arthritis & $2 \mathrm{C} \mathrm{L}$ \\
\hline Sibrotuzumab (45) & CDR-grafted mouse/human IgG1 & FAP & $\begin{array}{l}\text { Colorectal and non-small cell lung } \\
\text { cancer }\end{array}$ & $2 \mathrm{C} \mathrm{NL}+\mathrm{L}$ \\
\hline Trastuzumab (46) & CDR-grafted mouse/human IgG1 & HER2 & Breast cancer and solid tumors & $2 \mathrm{C} \mathrm{L}$ \\
\hline Ustekinumab (47) & Human IgG1 & IL-12 and IL-23 & Psoriasis & $1 \mathrm{C} \mathrm{L}$ \\
\hline
\end{tabular}

1C or 2C = one- or two-compartment model; $\mathbf{C D R}=$ complementarity determining region; $\mathbf{E G F R}=$ epidermal growth factor receptor; FAP = fibroblast activation protein; HER2 = human epidermal growth factor receptor 2; IGF-IR = insulin-like growth factor I receptor; $\mathbf{L}=$ model with linear elimination from the central compartment; $\mathbf{N L}=$ model with nonlinear elimination from the central compartment; $\mathbf{N L}+\mathbf{L}=$ model with parallel linear and nonlinear elimination pathways from the central compartment; $\mathbf{N L}+\mathbf{L}^{*}=$ model with nonlinear and linear elimination pathways from the central and peripheral compartments, respectively; RANKL = receptor activator of nuclear factor- $\kappa \mathrm{B}$ ligand; $\mathbf{R M C}=$ receptor-mediated clearance model; TAG-72 = tumor-associated glycoprotein-72; TCR $=$ T-cell receptor; TMDD $=$ target-mediated drug disposition model; TNF- $\alpha$ = tumor necrosis factor alpha; VEGF = vascular endothelial growth factor.

${ }^{\dagger}$ Population pharmacokinetic/pharmacodynamic analysis. 
Table 1-2. Population estimates and their between-subject variability [\%RSE or confidence interval ${ }^{*}{ }^{\wedge}$ ] of absorption related parameters."

\begin{tabular}{|c|c|c|c|c|c|}
\hline Antibody & $\mathbf{F}^{\dagger}$ & & F BSV $(\% C V)$ & $\mathrm{Ka}^{\dagger}\left(\mathrm{day}^{-1}\right)$ & Ka BSV $(\% C V)$ \\
\hline Efalizumab (21) & 0.564 & [5.3] & $51 \quad[13]$ & $0.242 \quad[9.4]$ & $44 \quad[12]$ \\
\hline Denosumab (24) & $0.747^{\mathrm{a}}$ & & & $0.132 \quad[4.9]$ & $\mathrm{NE}$ \\
\hline Denosumab (32) & $0.491^{\mathrm{b}}$ & & & $0.174 \quad[5.1]$ & 53 \\
\hline Omalizumab (41) & & & & $0.48 \quad[5.7]$ & 40 \\
\hline Efalizumab (33) & & & & {$\left[0.095-0.394^{\wedge}\right]$} & $\mathrm{NE}$ \\
\hline Ustekinumab (47) & & & & $0.354\left[0.277-0.499^{\wedge}\right]$ & $\mathrm{NE}$ \\
\hline
\end{tabular}

$\mathbf{B S V}=$ between-subject variability $; \mathbf{F}=$ absolute bioavailability $; \mathbf{K a}=$ absorption rate constant $\mathbf{N E}=$ not estimated; $\mathbf{\%} \mathbf{R S E}=$ percent relative standard error.

$* 90 \%$ or $\wedge 95 \%$ bootstrap confidence interval.

** Not available or not applicable estimates are blank.

${ }^{\dagger} \mathrm{F}$ and Ka were determined following subcutaneous administration.

a. $\theta_{\mathrm{F}}$ (theta estimate for calculation of bioavailability) (\%RSE) was 2.96 (23); $\theta_{\mathrm{F}}$ BSV was not estimated.

b. $\theta_{\mathrm{F}}(\% \mathrm{RSE})$ and $\theta_{\mathrm{F}} \mathrm{BSV}$ were $0.964(7.9)$ and $68 \% \mathrm{CV}$, respectively. 
Table 1-3. Population estimates and their between-subject variability [\%RSE or confidence interval ${ }^{*}$, ] of distribution related parameters.

\begin{tabular}{|c|c|c|c|c|c|c|c|c|c|}
\hline \multirow{2}{*}{$\begin{array}{l}\text { Antibody } \\
\text { Sibrotuzumab (45) }\end{array}$} & \multicolumn{2}{|l|}{$V_{1}(L)$} & \multicolumn{2}{|c|}{$V_{1}$ BSV $(\% C V)$} & \multicolumn{2}{|l|}{$V_{2}(L)$} & \multicolumn{2}{|c|}{$\mathrm{V}_{2} \mathrm{BSV}(\% \mathrm{CV})$} & \multirow{2}{*}{$\frac{\mathbf{V}_{\text {ss }}(\mathbf{L})}{7.32^{\ddagger}}$} \\
\hline & 4.13 & [3.7] & 20 & {$[52]$} & 3.19 & {$[8.8]$} & 20 & {$[52]$} & \\
\hline Matuzumab (40) & 3.72 & {$[3.0]$} & $22^{\mathrm{a}}$ & [20] & 1.84 & {$[9.0]$} & $62^{\mathrm{a}}$ & [28] & $5.56^{\ddagger}$ \\
\hline Efalizumab $^{\mathrm{i}}(35)$ & $5.14^{\mathrm{b}}$ & [8.6] & 39 & [33] & $4.18^{*}$ & & & & $9.32^{\ddagger}$ \\
\hline Efalizumab (21) & $4.50^{\mathrm{c}}$ & [3.5] & 52 & {$[9.0]$} & $2.26^{\star}$ & & & & $6.76^{\ddagger}$ \\
\hline Denosumab (24) & 2.38 & {$[7.4]$} & 16 & & $1.59^{\ddagger}$ & & & & $3.97^{\star}$ \\
\hline Denosumab (32) & 2.42 & {$[7.0]$} & 37 & & 1.27 & {$[5.8]$} & NE & & $3.69^{\ddagger}$ \\
\hline CP-751, 871 (31) & 4.02 & {$[4.0]$} & 22 & [50] & 6.82 & {$[24]$} & 92 & [49] & 10.84 \\
\hline Panitumumab (42) & 3.95 & [3.83-4.10*] & $34^{\mathrm{k}}$ & [28-39*] & 2.59 & {$[2.30-2.87 *]$} & 38 & {$[27-49 *]$} & $6.54^{\ddagger}$ \\
\hline Cetuximab (29) & 2.83 & [2.69-2.96*] & 19 & [13-22*] & 2.43 & {$\left[1.95-2.85^{*}\right]$} & 56 & {$[18-73 *]$} & $5.26^{\ddagger}$ \\
\hline Alemtuzumab (23) & 11.3 & {$\left[8.92-16.4^{\wedge}\right]$} & 84 & [21] & 41.5 & {$\left[21.3-73.2^{\wedge}\right]$} & 179 & [28] & $52.8^{\ddagger}$ \\
\hline Clenoliximab (30) & 3.58 & {$[8.5]$} & 37 & & $5.35^{\ddagger}$ & & & & $8.93^{\ddagger}$ \\
\hline Golimumab (34) & 3.07 & {$\left[2.63-3.51^{\wedge}\right]$} & 26 & [28] & 3.68 & {$\left[2.71-4.75^{\wedge}\right]$} & 45 & [40] & $6.75^{\ddagger}$ \\
\hline Inolimomab (38) & 2.76 & [26] & $68^{\mathrm{d}}$ & [20] & 2.25 & {$[18]$} & $\mathrm{NE}$ & & $5.01^{\ddagger}$ \\
\hline mAbF19 (39) & 3.13 & [11] & 37 & [49] & $1.74^{\ddagger, j}$ & & & & $4.87^{\mathrm{j}}$ \\
\hline $\mathrm{HuCC} 49 \Delta \mathrm{CH} 2(17)$ & 3.46 & [3.09-3.84*] & $23^{\mathrm{e}}$ & {$[10-34 *]$} & 2.50 & {$\left[1.52-3.69^{*}\right]$} & $\mathrm{NE}$ & & $5.96^{\ddagger}$ \\
\hline Rituximab (44) & 2.98 & {$\left[2.88-3.08^{\wedge}\right]$} & 12 & {$\left[9.5-14^{\wedge}\right]$} & 3.64 & & & & $6.62^{\ddagger}$ \\
\hline Trastuzumab (46) & 2.95 & {$\left[2.67-3.27^{\wedge}\right]$} & 29 & {$\left[21-38^{\wedge}\right]$} & $4.79^{*}$ & & & & 7.74 \\
\hline Pertuzumab (43) & 2.74 & {$\left[2.64-2.84^{\wedge}\right]$} & 16 & {$\left[13-19^{\wedge}\right]$} & $2.16^{\ddagger}$ & & & & $4.9^{\ddagger}$ \\
\hline Bevacizumab (28) & 2.66 & {$\left[2.57-2.76^{\wedge}\right]$} & 17 & {$\left[14-20^{\wedge}\right]$} & $2.76^{\ddagger}$ & & & & $5.42^{\ddagger}$ \\
\hline Infliximab (36) & 3.06 & {$\left[2.96-3.16^{\wedge}\right]$} & $18^{\mathrm{f}}$ & {$\left[16-19^{\wedge}\right]$} & 2.94 & {$\left[2.63-3.24^{\wedge}\right]$} & NE & & $6.0^{*}$ \\
\hline Infliximab (37) & 3.29 & {$\left[3.02-3.58^{\wedge}\right]$} & 22 & {$\left[18-26^{\wedge}\right]$} & 4.13 & {$\left[3.65-4.57^{\wedge}\right]$} & NE & & $7.42^{\ddagger}$ \\
\hline Basiliximab (26) & 3.72 & {$[1.2]$} & 41 & [18] & $4.08^{\star}$ & & & & $7.8^{\ddagger}$ \\
\hline Basiliximab (27) & 5.47 & {$[7.4]$} & 31 & [32] & $1.78^{\ddagger}$ & & & & $7.25^{\ddagger}$ \\
\hline ATM-027 (25) & 2.8 & [6.9] & $\mathrm{NE}$ & & 3.1 & [19] & 25 & [38] & $5.9^{*}$ \\
\hline Omalizumab (41) & $5.9^{g}$ & {$[1.8]$} & 13 & & & & & & \\
\hline Efalizumab (33) & $9.13^{\mathrm{g}}$ & {$\left[3.9-11.7^{\wedge}\right]$} & 29 & {$\left[<10^{-3}-44^{\wedge}\right]$} & & & & & \\
\hline Ustekinumab (47) & $15.7^{\mathrm{g}, \mathrm{h}}$ & {$\left[15.1-16.2^{\wedge}\right]$} & 33 & {$\left[28-38^{\wedge}\right]$} & & & & & \\
\hline
\end{tabular}


Table 1-3 continued.

\begin{tabular}{|c|c|c|c|c|}
\hline \multirow{2}{*}{$\begin{array}{l}\text { Antibody } \\
\text { Sibrotuzumab (45) }\end{array}$} & \multicolumn{2}{|c|}{ Q (L/day) } & \multicolumn{2}{|c|}{$\begin{array}{l}\text { Q BSV } \\
\text { (\%CV) }\end{array}$} \\
\hline & 0.902 & {$[9.6]$} & $\mathrm{NE}$ & \\
\hline Matuzumab (40) & 0.919 & [7.6] & $\mathrm{NE}$ & \\
\hline Efalizumab $^{\mathrm{i}}(35)$ & $2.46^{\ddagger}$ & & & \\
\hline Efalizumab (21) & $0.437^{\ddagger}$ & & & \\
\hline Denosumab (24) & $0.533^{\ddagger}$ & & & \\
\hline Denosumab (32) & 0.629 & [18] & $\mathrm{NE}$ & \\
\hline CP-751, $871(31)$ & 0.7 & [14] & 50 & [56] \\
\hline Panitumumab (42) & 0.390 & {$[0.323-0.475 *]$} & $\mathrm{NE}$ & \\
\hline Cetuximab (29) & 2.47 & {$[1.49-4.58 *]$} & 97 & {$\left[40-133^{*}\right]$} \\
\hline Alemtuzumab (23) & 25.2 & {$\left[10.6-51.1^{\wedge}\right]$} & $\mathrm{NE}$ & \\
\hline Clenoliximab (30) & $0.877^{\ddagger}$ & & & \\
\hline Golimumab (34) & 0.42 & {$\left[0.31-0.50^{\wedge}\right]$} & 45 & [52] \\
\hline Inolimomab (38) & 53.3 & {$[52]$} & $43^{\mathrm{d}}$ & [425] \\
\hline mAbF19 (39) & 3.41 & [18] & $\mathrm{NE}$ & \\
\hline $\mathrm{HuCC} 49 \Delta \mathrm{CH} 2(17)$ & 0.154 & [0.122-0.191*] & 39 & {$[24-54 *]$} \\
\hline Rituximab (44) & $0.656^{\ddagger}$ & & & \\
\hline Trastuzumab (46) & $0.484^{\ddagger}$ & & & \\
\hline Pertuzumab (43) & $0.556^{\ddagger}$ & & & \\
\hline Bevacizumab (28) & $0.593^{\ddagger}$ & & & \\
\hline Infliximab (36) & 1.72 & {$\left[1.14-2.6^{\wedge}\right]$} & $\mathrm{NE}$ & \\
\hline Infliximab (37) & 7.14 & {$\left[3.67-10.10^{\wedge}\right]$} & $\mathrm{NE}$ & \\
\hline Basiliximab (26) & $4.17^{\ddagger}$ & & & \\
\hline Basiliximab (27) & $0.569^{\ddagger}$ & & & \\
\hline ATM-027 (25) & 2.18 & [170] & $\mathrm{NE}$ & \\
\hline \multicolumn{5}{|l|}{ Omalizumab (41) } \\
\hline \multicolumn{5}{|l|}{ Efalizumab (33) } \\
\hline Ustekinumab (47) & & & & \\
\hline
\end{tabular}


Table 1-3 continued.

\begin{tabular}{|c|c|c|c|c|c|c|c|c|}
\hline Antibody & \multicolumn{2}{|c|}{$K_{12}\left(\operatorname{day}^{-1}\right)$} & \multicolumn{2}{|c|}{$\begin{array}{l}\mathrm{K}_{12} \mathrm{BSV} \\
(\% \mathrm{CV})\end{array}$} & \multicolumn{2}{|c|}{$K_{21}\left(\operatorname{day}^{-1}\right)$} & \multicolumn{2}{|c|}{$\begin{array}{l}\mathrm{K}_{21} \mathrm{BSV} \\
(\% \mathrm{CV}) \\
\end{array}$} \\
\hline Sibrotuzumab (45) & $0.218^{\ddagger}$ & & & & $0.283^{\ddagger}$ & & & \\
\hline Matuzumab (40) & $0.247^{\ddagger}$ & & & & $0.499^{\ddagger}$ & & & \\
\hline Efalizumab $^{\mathrm{i}}(35)$ & 0.478 & [24] & 112 & [53] & 0.588 & [11] & $\mathrm{NE}$ & \\
\hline Efalizumab (21) & 0.097 & [21] & 168 & [6.1] & 0.193 & [13] & 96 & [7.7] \\
\hline Denosumab (24) & 0.224 & [12] & $\mathrm{NE}$ & & 0.336 & [13] & NE & \\
\hline Denosumab (32) & $0.260^{\ddagger}$ & & & & $0.495^{\ddagger}$ & & & \\
\hline \multicolumn{9}{|l|}{ CP-751, 871 (31) } \\
\hline Panitumumab (42) & $0.0987^{\ddagger}$ & & & & $0.151^{\ddagger}$ & & & \\
\hline Cetuximab (29) & $0.873^{\ddagger}$ & & & & $1.02^{\ddagger}$ & & & \\
\hline Alemtuzumab (23) & $2.23^{\ddagger}$ & & & & $0.607^{\ddagger}$ & & & \\
\hline Clenoliximab (30) & 0.245 & {$[5.2]$} & 12 & & 0.164 & [21] & 28 & \\
\hline Golimumab (34) & $0.137^{\ddagger}$ & & & & $0.114^{\ddagger}$ & & & \\
\hline Inolimomab (38) & $19.3^{\ddagger}$ & & & & $23.7^{\ddagger}$ & & & \\
\hline mAbF19 (39) & $1.09^{\ddagger}$ & & & & $1.96^{\ddagger}$ & & & \\
\hline HuCC49 $\Delta \mathrm{CH} 2$ (17) & $0.0445^{\ddagger}$ & & & & $0.0616^{\ddagger}$ & & & \\
\hline Rituximab (44) & 0.22 & {$\left[0.20-0.25^{\wedge}\right]$} & NE & & 0.18 & {$\left[0.17-0.19^{\wedge}\right]$} & $\mathrm{NE}$ & \\
\hline Trastuzumab (46) & 0.164 & {$\left[0.135-0.191^{\wedge}\right]$} & 54 & {$\left[43-62^{\wedge}\right]$} & 0.101 & {$\left[0.0826-0.122^{\wedge}\right]$} & 67 & {$\left[57-78^{\wedge}\right]$} \\
\hline Pertuzumab (43) & 0.203 & {$\left[0.159-0.416^{\wedge}\right]$} & NE & & 0.258 & {$\left[0.203-0.480^{\wedge}\right]$} & 25 & {$\left[11-34^{\wedge}\right]$} \\
\hline Bevacizumab (28) & 0.223 & {$\left[0.134-0.501^{\wedge}\right]$} & $\mathrm{NE}$ & & 0.215 & {$\left[0.138-0.416^{\wedge}\right]$} & NE & \\
\hline Infliximab (36) & $0.562^{\ddagger}$ & & & & $0.585^{\ddagger}$ & & & \\
\hline Infliximab (37) & $2.170^{\ddagger}$ & & & & $1.729^{\ddagger}$ & & & \\
\hline Basiliximab (26) & 1.12 & [14] & NE & & 1.02 & [31] & 51 & [29] \\
\hline Basiliximab (27) & 0.104 & [38] & 68 & [45] & 0.319 & [25] & 94 & [33] \\
\hline ATM-027 (25) & $0.779^{\ddagger}$ & & & & $0.703^{\ddagger}$ & & & \\
\hline \multicolumn{9}{|l|}{ Omalizumab (41) } \\
\hline \multicolumn{9}{|l|}{ Efalizumab (33) } \\
\hline Ustekinumab (47) & & & & & & & & \\
\hline
\end{tabular}




\section{Table 1-3 continued.}

BSV = between-subject variability; $\mathbf{K}_{\mathbf{1 2}}$ and $\mathbf{K}_{\mathbf{2 1}}=$ distribution rate constants from central to peripheral compartment and vice versa, respectively; $\mathbf{N E}=$ not estimated; $\mathbf{Q}=$ intercompartmental clearance; $\mathbf{\%} \mathbf{R S E}=$ percent relative standard error; $\mathbf{V}_{\mathbf{1}}$ or $\mathbf{V}_{\mathbf{2}}$ $=$ volume of the central or peripheral compartment, respectively; $\mathbf{V}_{\mathbf{s s}}=$ volume of distribution at steady state.

$* 90 \%$ or $\wedge 95 \%$ bootstrap confidence interval.

** Not available or not applicable estimates are blank.

${ }^{*}$ Parameter derived from population estimates using the following equations: $\mathrm{CL}_{\mathrm{L}}$ or $\mathrm{CL}_{\mathrm{T}}=\mathrm{K}_{10} * \mathrm{~V}_{1} ; \mathrm{Q}=\mathrm{K}_{12} * \mathrm{~V}_{1}=\mathrm{K}_{21} * \mathrm{~V}_{2}$; $\mathrm{V}_{\mathrm{ss}}=\mathrm{V}_{1}+\mathrm{V}_{2}$.

a. Covariance of between-subject variability estimated; correlation coefficients: $\mathrm{V}_{1} \_\mathrm{V}_{2}(0.777), \mathrm{V}_{1} \_\mathrm{V}_{\max }(0.875), \mathrm{V}_{2} \mathrm{~V}_{\max }$ (0.875).

b. Calculated for a $70 \mathrm{~kg}$ patient using $\mathrm{V}_{1}(73.4 \mathrm{ml} / \mathrm{kg})$ and $\mathrm{V}_{\max }(39 \mu \mathrm{g} / \mathrm{kg} /$ day $)$ population estimates.

c. Calculated for a $70 \mathrm{~kg}$ patient using $\mathrm{V}_{1}(64.3 \mathrm{ml} / \mathrm{kg})$ and $\mathrm{V}_{\max }(26.9 \mu \mathrm{g} / \mathrm{kg} /$ day $)$ population estimates.

d. Covariance of between-subject variability estimated; correlation coefficients: CL_V 1 (-0.05), CL_Q (-0.76), V $\mathrm{V}_{-} \mathrm{Q}(-0.62)$.

e. Covariance of between-subject variability estimated; correlation coefficient: $C L-\bar{V}_{1}\left(0.729 ;\left[0.335-0.910^{*}\right]\right)$.

f. Covariance of between-subject variability estimated; correlation coefficient: $\mathrm{CL}_{-} \mathrm{V}_{1}\left(0.342 ;\left[0.214-0.456^{\wedge}\right]\right)$.

g. Apparent clearance or volume of distribution (i.e., CL/F or V/F).

h. Covariance of between-subject variability estimated; correlation coefficient: CL/F_V/F (0.817).

i. Results reported for $2 \mathrm{C} \mathrm{NL}+\mathrm{L}$ model; a receptor-mediated clearance model was also investigated.

j. $\mathrm{V}_{\mathrm{ss}}(\% \mathrm{RSE})$ and $\mathrm{V}_{\mathrm{ss}} \mathrm{BSV}(\% \mathrm{RSE})$ estimates were $4.87 \mathrm{~L}(9.2)$ and $31 \% \mathrm{CV}(45)$, respectively.

k. Covariance of between-subject variability estimated; correlation coefficient: $C_{2} V_{1}(0.662 ;[0.396-1.02 *])$. 
Table 1-4. Population estimates and their between-subject variability [\%RSE or confidence interval ${ }^{* \wedge}$ ] of clearance related parameters. ${ }^{* *}$

\begin{tabular}{|c|c|c|c|c|c|c|c|c|}
\hline \multirow{2}{*}{$\frac{\text { Antibody }}{\text { Sibrotuzumab (45) }}$} & \multicolumn{2}{|c|}{$\mathrm{V}_{\max }(\mathrm{mg} / \mathrm{day})$} & \multicolumn{2}{|c|}{$\begin{array}{l}V_{\max } B S V \\
(\% \mathrm{CV}) \\
\end{array}$} & \multicolumn{2}{|c|}{$K_{m}(\mathbf{m g} / \mathrm{L})$} & \multirow{2}{*}{$\begin{array}{l}\mathbf{K}_{\mathrm{m}} \mathbf{B S V} \\
(\% \mathrm{CV})\end{array}$} & \multirow{2}{*}{$\begin{array}{l}\begin{array}{l}C L_{\text {int }} \\
\text { (L/day) }\end{array} \\
3.70^{\ddagger}\end{array}$} \\
\hline & 0.811 & {$[25]$} & 29 & {$[50]$} & 0.219 & {$[57]$} & & \\
\hline Matuzumab (40) & 10.9 & {$[14]$} & $54^{\mathrm{a}}$ & {$[38]$} & 4.0 & {$[30]$} & $\mathrm{NE}$ & $2.73^{\ddagger}$ \\
\hline Efalizumab $^{\mathrm{i}}(35)$ & $2.73^{\mathrm{b}}$ & {$[11]$} & 42 & {$[51]$} & 0.0973 & {$[27]$} & $122[74]$ & $28.1^{\ddagger}$ \\
\hline Efalizumab (21) & $1.88^{\mathrm{c}}$ & {$[4.3]$} & 65 & {$[8.1]$} & 0.033 & [21] & $124 \quad[6.8]$ & $57.0^{\ddagger}$ \\
\hline Denosumab (24) & 0.0878 & {$[7.5]$} & 32 & & 0.164 & [11] & $\mathrm{NE}$ & $0.54^{\ddagger}$ \\
\hline Denosumab (32) & 0.0895 & {$[6.3]$} & 46 & & 0.192 & {$[7.5]$} & $\mathrm{NE}$ & $0.47^{\ddagger}$ \\
\hline CP-751, 871 (31) & 2.42 & {$[29]$} & 34 & [210] & 1.22 & {$[97]$} & [94] & $1.98^{\ddagger}$ \\
\hline Panitumumab (42) & 12.1 & [11.4-12.7*] & $29^{j}$ & [24-33*] & 0.426 & [0.364-0.586*] & $80^{\mathrm{j}} \quad\left[59-100^{*}\right]$ & $28.4^{\ddagger}$ \\
\hline Cetuximab (29) & 105 & [81.6-159*] & 15 & {$\left[12-19^{*}\right]$} & 74 & {$[38.2-163 *]$} & $\mathrm{NE}$ & $1.42^{\ddagger}$ \\
\hline Alemtuzumab (23) & 24.5 & {$\left[15.6-44.2^{\wedge}\right]$} & 32 & {$[32]$} & 0.338 & {$\left[0.226-0.849^{\wedge}\right]$} & $145[37]$ & $72.5^{\ddagger}$ \\
\hline Clenoliximab (30) & 35.8 & {$[4.2]$} & 47 & & 1.29 & {$[23]$} & $\mathrm{NE}$ & $27.8^{\ddagger}$ \\
\hline \multicolumn{9}{|l|}{ Golimumab (34) } \\
\hline \multicolumn{9}{|l|}{ Inolimomab (38) } \\
\hline \multicolumn{9}{|l|}{ mAbF19 (39) } \\
\hline \multicolumn{9}{|l|}{$\mathrm{HuCC} 49 \Delta \mathrm{CH} 2$ (17) } \\
\hline \multicolumn{9}{|l|}{ Rituximab (44) } \\
\hline \multicolumn{9}{|l|}{ Trastuzumab (46) } \\
\hline \multicolumn{9}{|l|}{ Pertuzumab (43) } \\
\hline \multicolumn{9}{|l|}{ Bevacizumab (28) } \\
\hline \multicolumn{9}{|l|}{ Infliximab (36) } \\
\hline \multicolumn{9}{|l|}{ Infliximab (37) } \\
\hline \multicolumn{9}{|l|}{ Basiliximab (26) } \\
\hline \multicolumn{9}{|l|}{ Basiliximab (27) } \\
\hline \multicolumn{9}{|l|}{ ATM-027 (25) } \\
\hline \multicolumn{9}{|l|}{ Omalizumab (41) } \\
\hline \multicolumn{9}{|l|}{ Efalizumab (33) } \\
\hline Ustekinumab (47) & & & & & & & & \\
\hline
\end{tabular}


Table 1-4 continued.

\begin{tabular}{|c|c|c|c|c|}
\hline \multirow{2}{*}{$\begin{array}{l}\text { Antibody } \\
\text { Sibrotuzumab (45) }\end{array}$} & \multicolumn{2}{|c|}{$\mathrm{CL}_{\mathrm{L}}(\mathrm{L} / \mathrm{day})$} & \multicolumn{2}{|c|}{$\begin{array}{l}C_{L} L_{L} \text { BSV } \\
(\% \mathrm{CV}) \\
\end{array}$} \\
\hline & 0.530 & {$[9.6]$} & 57 & [52] \\
\hline Matuzumab (40) & 0.348 & [4.1] & 24 & [21] \\
\hline Efalizumab $^{\mathrm{i}}(35)$ & $0.535^{\ddagger}$ & & & \\
\hline Efalizumab (21) & $0.513^{\ddagger}$ & & & \\
\hline Denosumab (24) & 0.066 & {$[7.2]$} & 25 & \\
\hline Denosumab (32) & 0.0710 & [4.5] & 32 & \\
\hline СР-751, 871 (31) & 0.243 & {$[8.3]$} & 59 & [34] \\
\hline Panitumumab (42) & 0.273 & {$\left[0.258-0.294^{*}\right]$} & $28^{\mathrm{k}}$ & {$\left[19-37^{*}\right]$} \\
\hline \multicolumn{5}{|l|}{ Cetuximab (29) } \\
\hline \multicolumn{5}{|l|}{ Alemtuzumab (23) } \\
\hline \multicolumn{5}{|l|}{ Clenoliximab (30) } \\
\hline \multicolumn{5}{|l|}{ Golimumab (34) } \\
\hline \multicolumn{5}{|l|}{ Inolimomab (38) } \\
\hline \multicolumn{5}{|l|}{ mAbF19 (39) } \\
\hline \multicolumn{5}{|l|}{$\mathrm{HuCC} 49 \Delta \mathrm{CH} 2(17)$} \\
\hline \multicolumn{5}{|l|}{ Rituximab (44) } \\
\hline \multicolumn{5}{|l|}{ Trastuzumab (46) } \\
\hline \multicolumn{5}{|l|}{ Pertuzumab (43) } \\
\hline \multicolumn{5}{|l|}{ Bevacizumab (28) } \\
\hline \multicolumn{5}{|l|}{ Infliximab (36) } \\
\hline \multicolumn{5}{|l|}{ Infliximab (37) } \\
\hline \multicolumn{5}{|l|}{ Basiliximab (26) } \\
\hline \multicolumn{5}{|l|}{ Basiliximab (27) } \\
\hline \multicolumn{5}{|l|}{ ATM-027 (25) } \\
\hline \multicolumn{5}{|l|}{ Omalizumab (41) } \\
\hline \multicolumn{5}{|l|}{ Efalizumab (33) } \\
\hline Ustekinumab (47) & & & & \\
\hline
\end{tabular}


Table 1-4 continued.

\begin{tabular}{|c|c|c|c|c|c|c|c|c|}
\hline Antibody & \multicolumn{2}{|c|}{$\mathbf{C} \mathbf{L}_{\mathrm{T}}$ (L/day) } & \multicolumn{2}{|c|}{$\begin{array}{l}\mathbf{C L}_{\mathrm{T}} \mathbf{B S V} \\
(\% \mathrm{CV})\end{array}$} & \multicolumn{2}{|l|}{$K_{10}\left(\right.$ day $\left.^{-1}\right)$} & \multicolumn{2}{|c|}{$\begin{array}{l}\mathrm{K}_{10} \mathrm{BSV} \\
(\% \mathrm{CV})\end{array}$} \\
\hline Sibrotuzumab (45) & & & & & $0.128^{\ddagger}$ & & & \\
\hline Matuzumab (40) & & & & & $0.0935^{\ddagger}$ & & & \\
\hline Efalizumab $^{\mathrm{i}}(35)$ & & & & & 0.104 & [17] & 21 & [99] \\
\hline Efalizumab (21) & & & & & 0.114 & [5.8] & 50 & [9.5] \\
\hline Denosumab (24) & & & & & $0.0277^{\ddagger}$ & & & \\
\hline Denosumab (32) & & & & & $0.0293^{\ddagger}$ & & & \\
\hline CP-751, 871 (31) & & & & & & & & \\
\hline Panitumumab (42) & & & & & $0.0691^{\ddagger}$ & & & \\
\hline Cetuximab (29) & & & & & & & & \\
\hline Alemtuzumab (23) & & & & & & & & \\
\hline Clenoliximab (30) & & & & & & & & \\
\hline Golimumab (34) & 0.40 & {$\left[0.33-0.49^{\wedge}\right]$} & 44 & [19] & $0.130^{\ddagger}$ & & & \\
\hline Inolimomab (38) & 1.85 & [19] & $43^{\mathrm{d}}$ & [87] & $0.670^{\ddagger}$ & & & \\
\hline mAbF19 (39) & 2.62 & {$[15]$} & 54 & [31] & $0.837^{\ddagger}$ & & & \\
\hline $\mathrm{HuCC} 49 \Delta \mathrm{CH} 2(17)$ & 1.53 & {$\left[1.36-1.71^{*}\right]$} & $28^{\mathrm{e}}$ & {$\left[20-35^{*}\right]$} & $0.442^{\ddagger}$ & & & \\
\hline Rituximab (44) & 0.257 & {$\left[0.246-0.271^{\wedge}\right]$} & 28 & {$\left[24-32^{\wedge}\right]$} & $0.0862^{\ddagger}$ & & & \\
\hline Trastuzumab (46) & 0.225 & {$\left[0.213-0.238^{\wedge}\right]$} & 43 & {$\left[39-47^{\wedge}\right]$} & $0.0763^{\ddagger}$ & & & \\
\hline Pertuzumab (43) & 0.214 & {$\left[0.201-0.228^{\wedge}\right]$} & 31 & {$\left[27-34^{\wedge}\right]$} & $0.0781^{\ddagger}$ & & & \\
\hline Bevacizumab (28) & 0.207 & {$\left[0.188-0.226^{\wedge}\right]$} & 26 & {$\left[23-31^{\wedge}\right]$} & $0.0778^{\ddagger}$ & & & \\
\hline Infliximab (36) & 0.273 & {$\left[0.259-0.288^{\wedge}\right]$} & $34^{\mathrm{f}}$ & {$\left[30-37^{\wedge}\right]$} & $0.0892^{\ddagger}$ & & & \\
\hline Infliximab (37) & 0.407 & {$\left[0.388-0.427^{\wedge}\right]$} & 38 & {$\left[35-41^{\wedge}\right]$} & $0.124^{\ddagger}$ & & & \\
\hline Basiliximab (26) & $0.882^{\ddagger}$ & & & & 0.237 & [19] & 35 & [26] \\
\hline Basiliximab (27) & $1.33^{\ddagger}$ & & & & 0.243 & [9.1] & 42 & [29] \\
\hline ATM-027 (25) & 0.190 & {$[6.7]$} & 28 & [24] & $0.0679^{\ddagger}$ & & & \\
\hline Omalizumab (41) & $0.176^{\mathrm{g}}$ & {$[2.1]$} & 20 & & & & & \\
\hline Efalizumab (33) & $1.29^{\mathrm{g}}$ & {$\left[1.17-1.39^{\wedge}\right]$} & 48 & {$\left[45-51^{\wedge}\right]$} & & & & \\
\hline Ustekinumab (47) & $0.465^{\mathrm{g}, \mathrm{h}}$ & {$\left[0.448-0.480^{\wedge}\right]$} & 41 & {$\left[38-44^{\wedge}\right]$} & & & & \\
\hline
\end{tabular}


Table 1-4 continued.

BSV = between-subject variability; $\mathbf{C} \mathbf{L}_{\mathbf{T}}=$ clearance; $\mathbf{C L}_{\mathbf{L}}=$ clearance of linear elimination pathway; $\mathbf{C} \mathbf{L}_{\text {int }}=$ intrinsic clearance (calculated as $\mathrm{V}_{\max } / \mathrm{K}_{\mathrm{m}}$ ); $\mathbf{K}_{\mathbf{1 0}}=$ elimination rate constant from central compartment; $\mathbf{K}_{\mathbf{m}}=$ concentration at halfmaximum elimination rate; $\mathbf{N E}=$ not estimated; $\% \mathbf{R S E}=$ percent relative standard error; $\mathbf{V}_{\mathbf{m a x}}=$ maximum elimination rate; $\mathbf{V}_{\mathbf{s s}}=$ volume of distribution at steady state.

$* 90 \%$ or $\wedge 95 \%$ bootstrap confidence interval.

** Not available or not applicable estimates are blank.

${ }^{\ddagger}$ Parameter derived from population estimates using the following equations: $\mathrm{CL}_{\mathrm{L}}$ or $\mathrm{CL}_{\mathrm{T}}=\mathrm{K}_{10} * \mathrm{~V}_{1} ; \mathrm{Q}=\mathrm{K}_{12} * \mathrm{~V}_{1}=\mathrm{K}_{21} * \mathrm{~V}_{2}$; $\mathrm{V}_{\mathrm{ss}}=\mathrm{V}_{1}+\mathrm{V}_{2}$.

a. Covariance of between-subject variability estimated; correlation coefficients: $\mathrm{V}_{1} \mathrm{~V}_{2}(0.777), \mathrm{V}_{1} \_\mathrm{V}_{\max }(0.875), \mathrm{V}_{2} \_\mathrm{V}_{\max }$ (0.875).

b. Calculated for a $70 \mathrm{~kg}$ patient using $\mathrm{V}_{1}(73.4 \mathrm{ml} / \mathrm{kg})$ and $\mathrm{V}_{\max }(39 \mu \mathrm{g} / \mathrm{kg} /$ day $)$ population estimates.

c. Calculated for a $70 \mathrm{~kg}$ patient using $\mathrm{V}_{1}(64.3 \mathrm{ml} / \mathrm{kg})$ and $\mathrm{V}_{\max }(26.9 \mu \mathrm{g} / \mathrm{kg} /$ day $)$ population estimates.

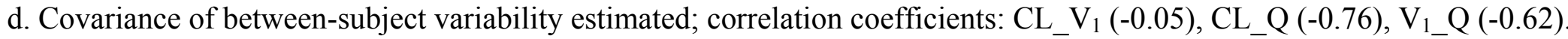

e. Covariance of between-subject variability estimated; correlation coefficient: $C L-\bar{V}_{1}\left(0.729 ;\left[0.335-0.910^{*}\right]\right)$.

f. Covariance of between-subject variability estimated; correlation coefficient: $\mathrm{CL}_{-} \mathrm{V}_{1}\left(0.342 ;\left[0.214-0.456^{\wedge}\right]\right)$.

g. Apparent clearance or volume of distribution (i.e., CL/F or V/F).

h. Covariance of between-subject variability estimated; correlation coefficient: CL/F_V/F (0.817).

i. Results reported for $2 \mathrm{C} \mathrm{NL}+\mathrm{L}$ model; a receptor-mediated clearance model was also investigated.

j. Covariance of between-subject variability estimated; correlation coefficient: $\mathrm{V}_{\max } \mathrm{K}_{\mathrm{m}}\left(0.461 ;\left[0.136-0.746^{*}\right]\right)$.

k. Covariance of between-subject variability estimated; correlation coefficient: $\mathrm{CL}_{-} \mathrm{V}_{1}(0.662 ;[0.396-1.02 *])$. 
Table 1-5. Identified covariates and their estimated effects on pharmacokinetic parameters ${ }^{* *}[\% \mathrm{RSE}$ or confidence interval $\left.{ }^{*}, \wedge\right]$.

\begin{tabular}{|c|c|c|c|c|c|c|c|}
\hline Antibody & & On $V_{\max }$ & & & On $\mathrm{CL}_{\mathrm{L}}$ & & Notes \\
\hline Sibrotuzumab (45) & $\mathrm{WGT}^{\mathrm{a}}$ & 0.00934 & [49] & $\mathrm{WGT}^{\mathrm{a}}$ & 0.0182 & [19] & \\
\hline Matuzumab (40) & & & & $\mathrm{WGT}^{\mathrm{a}}$ & 0.0087 & [28] & \\
\hline Denosumab (24) & $\mathrm{WGT}^{\mathrm{b}}$ & 0.6 & [27] & $\mathrm{WGT}^{\mathrm{b}}$ & 0.918 & [28] & \\
\hline CP-751, $871(31)$ & WGT $^{\mathrm{b}}$ & 1.11 & [74] & & & & \\
\hline \multirow[t]{5}{*}{ Panitumumab (42) } & WGT $^{b}$ & 0.621 & {$\left[0.488-0.725^{*}\right]$} & WGT $^{\mathrm{b}}$ & 0.411 & {$[0.261-0.572 *]$} & $\mathrm{CL}_{\mathrm{L}}$ was $23 \%$ slower in females \\
\hline & $\mathrm{AGE}^{\mathrm{b}}$ & -0.495 & {$\left[-0.688,-0.297^{*}\right]$} & $\mathrm{GDR}^{\mathrm{f}}$ & 0.769 & {$\left[0.721-0.818^{*}\right]$} & Patients with colorectal or renal \\
\hline & & & & $\operatorname{LUNG}^{\mathrm{f}}$ & 0.861 & {$[0.799-0.937 *]$} & cancer had a faster $\mathrm{CL}_{\mathrm{L}}$ than \\
\hline & & & & RENAL $^{\mathrm{f}}$ & 0.957 & {$[0.873-1.03 *]$} & patients with non-small cell lung \\
\hline & & & & OTHER $^{\mathrm{f}}$ & 0.870 & {$\left[0.792-0.956^{*}\right]$} & cancer or other types of cancer \\
\hline \multirow[t]{2}{*}{ Cetuximab (29) } & $\mathrm{IBW}^{\mathrm{a}}$ & 0.0108 & {$[0.0077-0.0139 *]$} & & & & \\
\hline & $\mathrm{WBC}^{\mathrm{a}}$ & 0.0216 & [0.0169-0.0296*] & & & & \\
\hline \multirow[t]{2}{*}{ Alemtuzumab (23) } & $\mathrm{WBC}^{\mathrm{b}}$ & 0.194 & {$\left[0.081-0.382^{\wedge}\right]$} & & & & \\
\hline & \multicolumn{7}{|c|}{ On $\mathrm{CL}_{\mathbf{T}}$} \\
\hline \multicolumn{8}{|l|}{ Golimumab (34) } \\
\hline $\mathrm{HuCC} 49 \Delta \mathrm{CH} 2$ (17) & & & & $\mathrm{WGT}^{\mathrm{c}}$ & 0.75 fixed & & \\
\hline \multirow[t]{2}{*}{ Rituximab (44) } & & & & $\mathrm{BSA}^{\mathrm{b}}$ & 1.02 & {$\left[0.54-1.64^{\wedge}\right]$} & CL was $39 \%$ faster in males \\
\hline & & & & $\mathrm{GDR}^{\mathrm{e}}$ & 0.39 & {$\left[0.17-0.63^{\wedge}\right]$} & \\
\hline \multirow[t]{2}{*}{ Trastuzumab (46) } & & & & $\mathrm{MET}^{\mathrm{e}}$ & 0.221 & {$\left[0.0611-0.429^{\wedge}\right]$} & CL was $22 \%$ faster in patients \\
\hline & & & & $\mathrm{ECD}^{\mathrm{b}}$ & 0.041 & {$\left[0.013-0.071^{\wedge}\right]$} & $\begin{array}{l}\text { with four or more metastatic } \\
\text { sites }\end{array}$ \\
\hline \multirow[t]{3}{*}{ Pertuzumab (43) } & & & & $\mathrm{WGT}^{\mathrm{b}}$ & 0.587 & {$\left[0.372-0.826^{\wedge}\right]$} & \\
\hline & & & & $\mathrm{ALB}^{\mathrm{b}}$ & -1.01 & {$\left[-1.42,-0.632^{\wedge}\right]$} & \\
\hline & & & & $\mathrm{ALKP}^{\mathrm{b}}$ & 0.169 & {$\left[0.067-0.258^{\wedge}\right]$} & \\
\hline
\end{tabular}


Table 1-5 continued.

\begin{tabular}{|c|c|c|c|c|c|}
\hline Antibody & On $V_{\max }$ & & On $\mathbf{C L}_{1}$ & & $\begin{array}{r}\text { Notes } \\
\end{array}$ \\
\hline \multirow[t]{6}{*}{ Bevacizumab (28) } & & $\mathrm{WGT}^{\mathrm{b}}$ & 0.368 & {$\left[0.088-0.650^{\wedge}\right]$} & CL was $26 \%$ faster in males \\
\hline & & $\mathrm{GDR}^{\mathrm{e}}$ & 0.264 & {$\left[0.132-0.398^{\wedge}\right]$} & $\mathrm{CL}$ in concomitant bolus-IFL \\
\hline & & $\mathrm{ALB}^{\mathrm{b}}$ & 0.726 & {$\left[0.472-1.02^{\wedge}\right]$} & regimen was $17 \%$ slower \\
\hline & & $\mathrm{ALKP}^{\mathrm{b}}$ & 0.133 & {$\left[0.070-0.206^{\wedge}\right]$} & compared to other concomitant \\
\hline & & $\mathrm{AST}^{\mathrm{b}}$ & 0.0715 & {$\left[0.002-0.144^{\wedge}\right]$} & chemotherapy regimens \\
\hline & & $\mathrm{CHEM}^{\mathrm{e}}$ & 0.174 & {$\left[0.096-0.244^{\wedge}\right]$} & combined \\
\hline \multirow[t]{3}{*}{ Infliximab (36) } & & $W_{B C}^{d}$ & 0.0106 & {$\left[0.0051-0.0162^{\wedge}\right]$} & CL was $42 \%$ faster for patients \\
\hline & & $\mathrm{IR}^{\mathrm{e}}$ & 0.419 & {$\left[0.215-0.662^{\wedge}\right]$} & positive for antibodies against \\
\hline & & & & & infliximab \\
\hline \multirow[t]{4}{*}{ Infliximab (37) } & & $\mathrm{ALB}^{\mathrm{b}}$ & -1.54 & {$\left[-1.94,-1.17^{\wedge}\right]$} & CL was $47 \%$ faster for patients \\
\hline & & $\operatorname{IR}^{\mathrm{e}}$ & 0.471 & {$\left[0.28-0.831^{\wedge}\right]$} & positive for antibodies against \\
\hline & & $\mathrm{GDR}^{\mathrm{e}}$ & -0.236 & {$\left[-0.291,-0.181^{\wedge}\right]$} & infliximab \\
\hline & & & & & CL was $24 \%$ slower in females \\
\hline \multicolumn{6}{|l|}{ ATM-027 (25) } \\
\hline Omalizumab (41) & & $\mathrm{WGT}^{\mathrm{b}}$ & 0.911 & {$[15]$} & \\
\hline \multirow[t]{6}{*}{ Efalizumab (33) } & & $\mathrm{WGT}^{\mathrm{b}}$ & 0.754 & {$\left[0.511-0.956^{\wedge}\right]$} & Obese patients $(\mathrm{BMI} \geq 30)$ had a \\
\hline & & $\mathrm{AGE}^{\mathrm{b}}$ & 0.218 & {$\left[0.091-0.344^{\wedge}\right]$} & $10 \%$ faster $\mathrm{CL} / \mathrm{F}$ \\
\hline & & $\mathrm{LYM}^{\mathrm{b}}$ & 0.165 & {$\left[0.065-0.274^{\wedge}\right]$} & Subjects receiving $2 \mathrm{mg} / \mathrm{kg}$ had \\
\hline & & $\mathrm{PASI}^{\mathrm{b}}$ & 0.220 & {$\left[0.132-0.312^{\wedge}\right]$} & a $24 \%$ slower CL/F vs. the \\
\hline & & $\mathrm{OBS}^{\mathrm{e}}$ & 0.0997 & {$\left[0.015-0.211^{\wedge}\right]$} & $1 \mathrm{mg} / \mathrm{kg}$ group \\
\hline & & $\operatorname{DOSE}^{\mathrm{e}}$ & -0.240 & {$\left[-0.292,-0.174^{\wedge}\right]$} & \\
\hline \multirow[t]{7}{*}{ Ustekinumab (47) } & & $\mathrm{WGT}^{\mathrm{b}}$ & 0.840 & {$\left[0.731-0.956^{\wedge}\right]$} & $\mathrm{CL} / \mathrm{F}$ was $28.7 \%$ faster in \\
\hline & & $\mathrm{DM}^{\mathrm{e}}$ & 0.287 & {$\left[0.189-0.377^{\wedge}\right]$} & patients with diabetes \\
\hline & & $\mathrm{IR}^{\mathrm{e}}$ & 0.355 & {$\left[0.172-0.540^{\wedge}\right]$} & $\mathrm{CL} / \mathrm{F}$ was $35.5 \%$ faster for \\
\hline & & $\mathrm{ALB}^{\mathrm{b}}$ & -0.896 & {$\left[-1.09,-0.673^{\wedge}\right]$} & patients positive for antibodies \\
\hline & & $\mathrm{CRCL}^{\mathrm{b}}$ & 0.188 & {$\left[0.128-0.251^{\wedge}\right]$} & against ustekinumab \\
\hline & & $\mathrm{GDR}^{\mathrm{e}}$ & 0.059 & {$\left[0.030-0.093^{\wedge}\right]$} & $\mathrm{CL} / \mathrm{F}$ was $5.9 \%$ faster for \\
\hline & & $\mathrm{ALKP}^{\mathrm{b}}$ & 0.113 & {$\left[0.065-0.161^{\wedge}\right]$} & females \\
\hline
\end{tabular}


Table 1-5 continued.

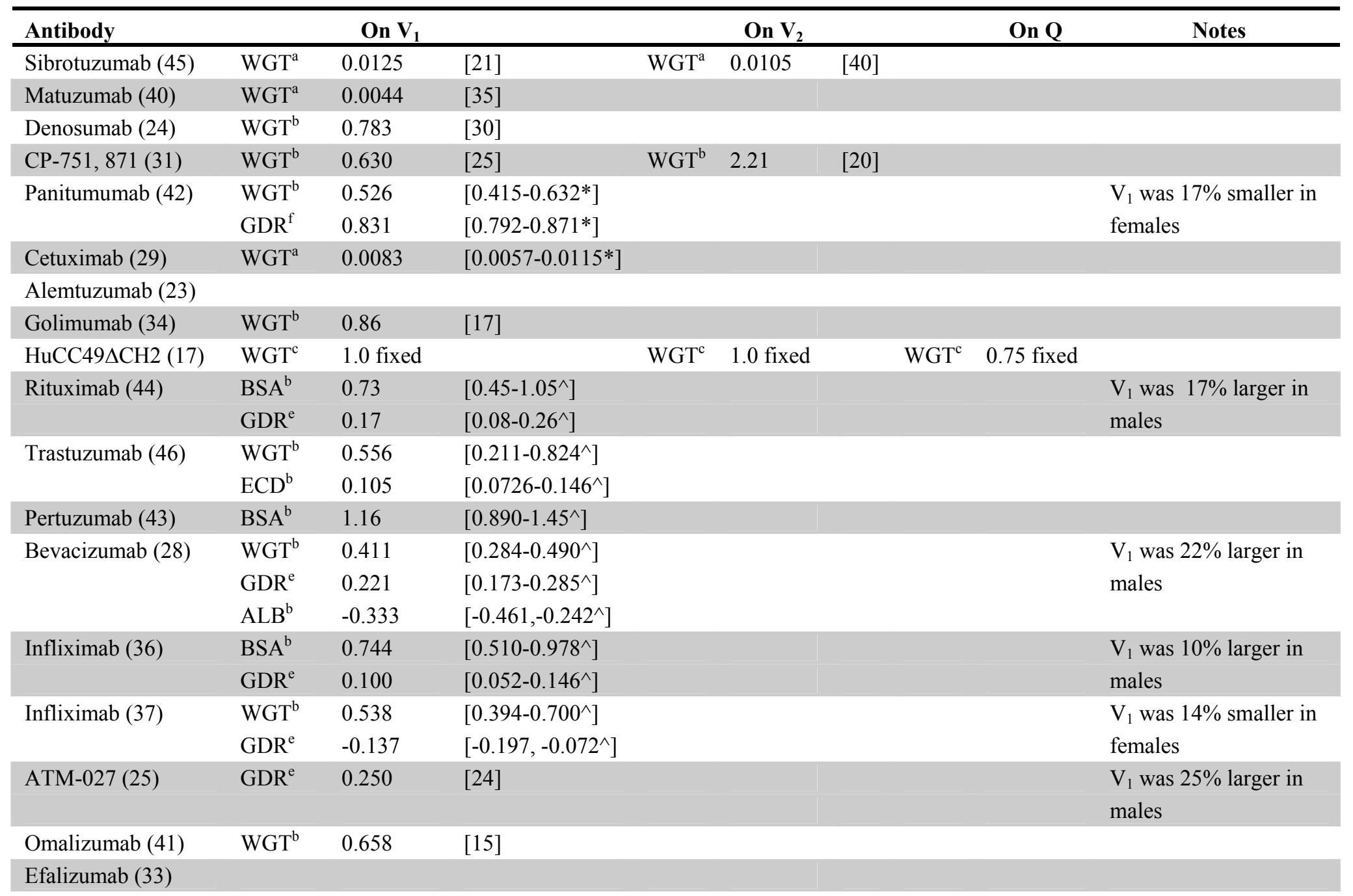


Table 1-5 continued.

\begin{tabular}{|c|c|c|c|c|c|}
\hline Antibody & & On $V_{1}$ & On $V_{2}$ & On $\mathbf{Q}$ & Notes \\
\hline \multirow[t]{4}{*}{ Ustekinumab (47) } & WGT $^{\mathrm{b}}$ & 0.807 & {$\left[0.707-0.905^{\wedge}\right]$} & & $\mathrm{V} / \mathrm{F}$ was $13.2 \%$ larger \\
\hline & $\mathrm{DM}^{\mathrm{e}}$ & 0.132 & {$\left[0.045-0.223^{\wedge}\right]$} & & in patients with \\
\hline & $\mathrm{RACE}^{\mathrm{e}}$ & -0.111 & {$\left[-0.154,-0.070^{\wedge}\right]$} & & diabetes \\
\hline & & & & & $\begin{array}{l}\mathrm{V} / \mathrm{F} \text { was } 11.1 \% \text { smaller } \\
\text { for non-Caucasians } \\
\text { vs. Caucasians }\end{array}$ \\
\hline
\end{tabular}


Table 1-5 continued.

AGE $=$ age; $\mathbf{A L B}=$ albumin; ALKP $=$ alkaline phosphatase; AST $=$ aspartate aminotransferase; $\mathbf{B M I}=$ body mass index; $\mathbf{B S A}=$ body surface area; $\mathbf{C H E M}=$ concomitant chemotherapies; $\mathbf{C} \mathbf{L}_{\mathbf{T}}=$ total clearance; $\mathbf{C L}_{\mathbf{L}}=$ clearance of linear elimination pathway; $\mathbf{C L} / \mathbf{F}=$ apparent clearance; $\mathbf{C O V}$ ref $=$ reference/typical covariate value; $\mathbf{C R C L}=$ creatinine clearance; $\mathbf{D M}=$ diabetes comorbidity; DOSE $=$ dose group; $\mathbf{E C D}=$ serum level of shed HER2 receptor extracellular domain; $\mathbf{F}=$ female; GDR = gender; IBW = ideal body weight; $\mathbf{I F L}=$ irinotecan, 5-fluorouracil, leucovorin regimen; IR =immune response with development of antibodies to the therapeutic monoclonal antibody; LUNG = non-small cell lung cancer; LYM = lymphocyte count; $\mathbf{M}=$ male; $\mathbf{M E T}=$ number of metastatic sites; $\mathbf{O B S}=$ obesity; OTHER $=$ other cancer types (see referenced paper for details); PASI = psoriasis area and severity index score; $\mathbf{Q}=$ intercompartmental clearance; $\mathbf{R E N A L}=$ renal cancer; $\% \mathbf{R S E}=$ percent relative standard error; $\mathbf{V}_{\mathbf{1}}$ or $\mathbf{V}_{\mathbf{2}}=$ volume of the central or peripheral compartment, respectively; $\mathbf{V} / \mathbf{F}=$ apparent volume of distribution; $\mathbf{V}_{\mathbf{m a x}}=$ maximum elimination rate; $\mathbf{W B C}=$ white blood cell count; $\mathbf{W G T}$ $=$ body weight.

* $90 \%$ or ${ }^{\wedge} 95 \%$ bootstrap confidence interval.

** Not available or not applicable estimates are blank.

Continuous covariate models:

a. Linear proportional (covariate centered); $\mathrm{P}=\theta_{1} *\left(1+\theta_{\mathrm{COV}} *(\mathrm{COV}-\mathrm{COV} r \mathrm{ref})\right.$ ).

b. Power (covariate normalized); $\mathrm{P}=\theta_{1} *(\mathrm{COV} / \mathrm{COV} r e f) * * \theta_{\mathrm{COV}}$.

c. Allometric (covariate normalized); $\theta_{\mathrm{COV}}$ in power model fixed to 0.75 or 1.0 .

d. Linear additive (covariate centered); $\mathrm{P}=\theta_{1}+\theta_{\mathrm{COV}} *$ (COV-COVref).

Categorical covariate models:

e. Linear proportional; $\mathrm{P}=\theta_{1} *\left(1+\theta_{\mathrm{COV}} * \mathrm{COV}\right)$ where $\mathrm{COV}$ is 0 or 1 , for example.

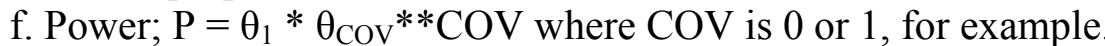


Table 1-6. Residual variability estimates ${ }^{* *}\left[\% \mathrm{RSE}\right.$ or confidence interval $\left.{ }^{*}{ }^{\wedge}\right]$.

\begin{tabular}{|c|c|c|c|c|}
\hline \multirow{2}{*}{$\begin{array}{l}\text { Antibody } \\
\text { Sibrotuzumab (45) }\end{array}$} & \multicolumn{2}{|c|}{ Proportional error $(\% \mathrm{CV})$} & \multicolumn{2}{|c|}{ Additive error (mg/L) } \\
\hline & 9.3 & {$[6.8]$} & 0.0491 & {$[19]$} \\
\hline Matuzumab (40) & 13.4 & {$[1.5]$} & 0.312 fixed & \\
\hline Efalizumab $^{\mathrm{b}}(35)$ & 11.7 & {$[21]$} & & \\
\hline Efalizumab (21) & 35.9 & [1.5] & & \\
\hline Denosumab (24) & 35.9 & & & \\
\hline Denosumab (32) & & & $2.66^{\mathrm{E}-04}$ & \\
\hline CP-751, $871(31)$ & 26 & [6.9] & & \\
\hline Panitumumab (42) & 42.0 & {$[36.3-48.4 *]$} & 0.03 & {$[0.005-0.05 *]$} \\
\hline Cetuximab (29) & $\begin{array}{l}14.6^{\mathrm{a}} \\
21.2^{\mathrm{a}}\end{array}$ & $\begin{array}{l}{[12.3-16.8 *]} \\
{[18.8-23.7 *]}\end{array}$ & & \\
\hline Alemtuzumab (23) & 37.2 & {$\left[31.0-40.0^{\wedge}\right]$} & 0.0647 & {$\left[0.0372-0.114^{\wedge}\right]$} \\
\hline Clenoliximab (30) & 8.8 & [29] & & \\
\hline Golimumab (34) & 15.0 & [23] & & \\
\hline Inolimomab (38) & 30.2 & [7] & & \\
\hline mAbF19 (39) & 12.5 & {$[31]$} & & \\
\hline $\mathrm{HuCC} 49 \Delta \mathrm{CH} 2(17)$ & 15.1 & {$[12.5-17.6 *]$} & $19.1 \mathrm{dps} / \mathrm{ml}$ & {$[12.7-29.3 *]$} \\
\hline Rituximab (44) & 19.0 & {$\left[17.2-21.3^{\wedge}\right]$} & 0.54 & {$\left[0.4-0.64^{\wedge}\right]$} \\
\hline Trastuzumab (46) & 23 & {$\left[21-24^{\wedge}\right]$} & & \\
\hline Pertuzumab (43) & 19.2 & {$\left[17.3-21.2^{\wedge}\right]$} & 2.27 & {$\left[0.002-4.16^{\wedge}\right]$} \\
\hline Bevacizumab (28) & 17.2 & {$\left[15.8-19.0^{\wedge}\right]$} & 7.2 & {$\left[0.093-10.3^{\wedge}\right]$} \\
\hline Infliximab (36) & 22.9 & {$\left[21.8-24.0^{\wedge}\right]$} & 2.89 & {$\left[2.48-3.22^{\wedge}\right]$} \\
\hline Infliximab (37) & 40.3 & {$\left[38.3-42.2^{\wedge}\right]$} & 0.0413 & {$\left[0.0368-0.0497^{\wedge}\right]$} \\
\hline Basiliximab (26) & 29.8 & {$[35]$} & 0.371 & {$[51]$} \\
\hline Basiliximab (27) & 18.4 & [18] & 0.084 & [23] \\
\hline ATM-027 (25) & 26.0 & [9.3] & 0.0084 & [15] \\
\hline Omalizumab (41) & 16.7 & & & \\
\hline Efalizumab (33) & 26.3 & {$\left[22.2-29.8^{\wedge}\right]$} & 1.58 & {$\left[1.01-2.07^{\wedge}\right]$} \\
\hline Ustekinumab (47) & 25.6 & & 0.085 fixed & \\
\hline
\end{tabular}


Table 1-6 continued.

\%RSE $=$ relative standard error.

$* 90 \%$ or ${ }^{\wedge} 95 \%$ bootstrap confidence interval.

** Not available or not applicable estimates are blank.

a. Separate residual error models used per each clinical trial.

b. Results reported for $2 \mathrm{C} \mathrm{NL}+\mathrm{L}$ model; a receptor-mediated clearance model was also investigated. 


\section{Antibody Absorption}

Most mAbs that are approved or in development are administered intravenously (IV), although other routes including subcutaneous (e.g., omalizumab, efalizumab, adalimumab) and intramuscular (e.g., palivizumab) administration are used. Oral administration of mAbs has been limited due to their large molecular weight and polarity, as well as their susceptibility to denaturation and proteolytic degradation in the gastrointestinal tract.

While the mechanism of absorption following subcutaneous (SC) or intramuscular administration (IM) is not completely understood, it has been suggested that $\mathrm{mAbs}$ are primarily absorbed via the lymphatic system. This presumption is supported in part from a study in which the lymphatic absorption of 5-fluoro-2'deoxyuridine (molecular weight $0.246 \mathrm{kDa})$, inulin $(5.2 \mathrm{kDa})$, cytochrome c $(12.3 \mathrm{kDa})$, and human recombinant interferon alpha-2a $(19 \mathrm{kDa})$, was investigated following SC administration in sheep (48). A positive linear relationship was observed between the molecular weight of the compound and the proportion of the dose absorbed via the lymphatics. Molecules larger than $16 \mathrm{kDa}$ predominantly underwent lymphatic absorption, while those under $1 \mathrm{kDa}$ were primarily absorbed by the blood capillaries. The molecular weights of the investigated compounds are much smaller compared to that of IgG and intact therapeutic mAbs of the IgG type $(\sim 150 \mathrm{kDa})$, so extrapolation of the observed linear relationship to mAbs should be made with caution. A sheep model was also used in another study to investigate the contribution of the lymphatics to the absorption and systemic availability of recombinant human erythropoietin alpha (30.4 $\mathrm{kDa}$ ) following SC administration (49). This study reported an $84 \%$ and $75 \%$ recovery of the administered dose in the peripheral and central lymph, respectively. Contrasting results to the above studies in sheep were reported in an investigation of lymphatic absorption of bovine insulin $(5.6 \mathrm{kDa})$, bovine albumin $(66 \mathrm{kDa})$, and recombinant human erythropoietin alpha $(30.4 \mathrm{kDa})$, following SC administration in a rat model (50). The investigators found that for all three macromolecules, less than $3 \%$ of the administered SC dose was recovered in the lymph. This study was conducted in rats which may not be a good model for human SC absorption, so extrapolation of the results to humans should be done with discretion. Drawing any conclusions about the lymphatic absorption of IgG molecules based on the results of the aforementioned studies should by done with caution since IgG molecules were not specifically investigated. There is still a great deal of uncertainty regarding the physiological mechanisms involved in the absorption of IgG following SC or IM administration, and in some aspects these mechanisms may differ from other macromolecules, for example the potential involvement of FcRn in this process (9).

Lymphatic absorption is believed to occur via convection, a process in which the $\mathrm{mAb}$ is "pulled" along by the flow of interstitial fluid into the highly permeable lymphatic capillaries where it is eventually returned back into the systemic circulation. Prior to reaching the systemic circulation, proteolytic degradation may occur at the injection site or during lymphatic transport, thereby reducing the bioavailable fraction of the antibody (9). The flow of lymph through the lymphatic vessels is relatively slow, for 
example, the lymph flow rate is approximately $1-2 \mathrm{ml} / \mathrm{kg} / \mathrm{hr}$ for the thoracic duct (51). As a result, absorption of the mAb can take place over several days. Typical values for time to reach maximal concentration following IM or SC administration range from 1-8 days, and absolute bioavailabilities generally range from $50-100 \%(6,7)$.

The majority of the population PK studies were performed using concentration data following IV administration, but SC data was available in a small number of studies. These studies are listed in Table 1-2 along with population estimates and between-subject variabilities for absolute bioavailability $(\mathrm{F})$ and absorption rate constant $(\mathrm{Ka})$. The median (range) population estimate for Ka was 0.217 day $^{-1}(0.132-0.48)$. Three different studies reported estimates for $F$ which included values of $49.1,56.4$, and $74.7 \%$. These population estimates for $\mathrm{F}$ and $\mathrm{Ka}$ are in agreement with the general absorption characteristics of $\mathrm{mAbs}$ following IM or SC dosing. Estimates of between-subject variability in $\mathrm{F}$ and $\mathrm{Ka}$ were large with values ranging from 40-53 \% CV (Table 1-2), although variability in these two parameters was not often estimated.

\section{Antibody Distribution}

The ability of mAbs to cross cell membranes is significantly hindered by their large molecular weight and hydrophilicity/polarity. Given this, mAbs often exhibit a small volume of distribution, and are believed to be largely confined to the vascular and interstitial spaces. Other factors that influence the distribution characteristics of antibodies include the distribution and density of the antibody target and morphology of the vascular capillaries (i.e., continuous, fenestrated, and sinusoidal). The movement of mAbs across or between cell membranes may occur via transcellular or paracellular mechanisms, respectively $(7,52,53)$.

The paracellular movement of mAbs occurs by means of convection, which is simply the transport of mAbs within the movement of fluid flow. This fluid flow is set up by the natural physiological exchange of fluids between the capillaries, interstitial space, and lymphatics. Convection is believed to be the primary mechanism responsible for the movement of mAbs from the vasculature to the interstitial space (9). Convection also plays a role in the distribution of mAbs within the interstitial fluid, and facilitates the transport of antibody out of the tissue as interstitial fluid enters the lymphatic capillaries. Given that the paracellular pores of the lymphatic capillaries are much larger than those in the vascular endothelium, it is assumed that the convective clearance of mAbs from the tissue is much more efficient than the process of convective extravasation, thereby maintaining relatively low $\mathrm{mAb}$ concentrations in the interstitial fluid $(7,9)$.

The transcellular movement of mAbs can occur via passive diffusion or endocytosis. However, given the physiochemical properties of mAbs, it is likely that passive diffusion does not play a significant role in their distribution. Endocytosis of mAbs can take place through three main processes: phagocytosis, fluid-phase pinocytosis, and receptor-mediated endocytosis (6). Virtually all cells in the body have the ability to take up proteins and other macromolecules including mAbs from the 
surrounding fluid space via fluid-phase pinocytosis. Receptor-mediated endocytosis may take place following binding of the mAb to a cell surface antigen or Fcy receptors (7).

Another potential mechanism for extravasation of mAbs is transcytosis by vascular endothelial cells $(7,52,54,55)$. Following fluid-phase pinocytosis by endothelial cells, mAbs within the intracellular vesicle may bind to FcRn, which not only protects the $\mathrm{mAb}$ from degradation, but eventually the mAb is recycled back into systemic circulation or transcytosed into the interstitial space (54). The FcRn-mediated transcytosis of IgG has been demonstrated in both endothelial and epithelial cells (56-62). However, the importance of FcRn-mediated transcytosis of IgG in the distribution of mAbs remains to be elucidated.

Following IV administration, the concentration-time profile of mAbs often follows a bi-exponential decline, which can be best described using a two-compartment PK model $(11,63)$. Accordingly, a two-compartment model was used in the majority of the $\mathrm{mAb}$ population $\mathrm{PK}$ analyses (Table 1-1). In the few studies where a onecompartment PK model was used, concentration data was only available following SC administration. The distribution phase of the mAbs in these studies may have been masked by a slow absorption phase often observed for mAbs following SC administration. An attempt was made in the population PK analyses of pertuzumab and inolimomab to fit a three-compartment model to the concentration data, but this resulted in an overparameterized model and did not show a significant improvement in fit versus a two-compartment model $(38,43)$.

Population estimates and between-subject variabilities for distribution related PK parameters are summarized in Table 1-3. Estimates for the volume of the central $\left(\mathrm{V}_{1}\right)$ and peripheral $\left(\mathrm{V}_{2}\right)$ compartments were quite similar for the different mAbs with the exception of alemtuzumab. Compared to other mAbs, alemtuzumab not only had a much larger $\mathrm{V}_{1}$ and $\mathrm{V}_{2}(11.3 \mathrm{~L}$ and $41.5 \mathrm{~L}$, respectively), but between-subject variability in both of these parameters was also larger $(84 \% \mathrm{CV}$ and $179 \% \mathrm{CV}$, respectively) (23). It is uncertain as to why these population parameter estimates for alemtuzumab are in stark contrast to what was observed for many of the other therapeutic mAbs. The median (range) estimate for $\mathrm{V}_{1}$ and $\mathrm{V}_{2}$ based on studies that used a two-compartment PK model (excluding alemtuzumab) was $3.1 \mathrm{~L}$ (2.4-5.5) and $2.8 \mathrm{~L}$ (1.3-6.8), respectively. In general, the estimates for $\mathrm{V}_{1}$ were approximately equal to plasma volume. Betweensubject variability was quantified for $\mathrm{V}_{1}$ in all but one of the population PK studies (Table 1-3). The between-subject variability in $\mathrm{V}_{1}$ was usually moderate, as the median (range) across all of the studies was $26 \% \mathrm{CV}(12-84)$. The values for volume of distribution at steady state $\left(\mathrm{V}_{\mathrm{ss}}=\mathrm{V}_{1}+\mathrm{V}_{2}\right)$ suggest an apparently limited distribution outside of the vascular space, and are consistent with the behavior of endogenous IgG. Based on data from early IgG metabolism studies in humans (64), the mean serum IgG concentration and total body IgG pool were $12 \mathrm{~g} / \mathrm{L}$ and $1.06 \mathrm{~g} / \mathrm{kg}$ body weight, respectively, which for a $70 \mathrm{~kg}$ person equates to a volume of distribution of around $6.2 \mathrm{~L}$ for IgG. Population estimates for intercompartmental clearance (Q) and the distribution rate constants $\left(\mathrm{K}_{12}\right.$ and $\left.\mathrm{K}_{21}\right)$ appeared to be less homogenous across the different $\mathrm{mAbs}$ compared to $V_{1}$ and $V_{2}$. In general, the estimates for $Q, K_{12}$, and $K_{21}$, suggested a slow 
transfer of $\mathrm{mAb}$ between the central and peripheral compartments. The median $\mathrm{Q}$ across all of the studies that used a two-compartment model was $0.79 \mathrm{~L} /$ day. Between-subject variability in $\mathrm{V}_{2}, \mathrm{Q}, \mathrm{K}_{12}$, and $\mathrm{K}_{21}$ was often not estimated in the population PK models. When estimated, between-subject variability in these parameters was usually moderate to high. Volume of distribution corrected for $\mathrm{SC}$ bioavailability $\left(\mathrm{V}_{1} / \mathrm{F}\right)$ was estimated in the population PK analyses of omalizumab, efalizumab, and ustekinumab, which have a bioavailability $\mathrm{F}$ following SC dosing of around $62 \%, 50 \%$, and $57 \%$, respectively $(33$, 41, 47). After adjusting for F, the volume of distribution for omalizumab, efalizumab, and ustekinumab are approximately 3.7, 4.6, and 9.0 L, respectively, which is in line with the results for other mAbs.

When estimating $\mathrm{V}_{\text {ss }}$ using a noncompartmental analysis or by fitting a mammillary compartmental model to the data, the assumption is made that elimination of the drug occurs at a site(s) in rapid equilibrium with plasma or the central compartment $(63,65)$. For some mAbs this assumption may be valid. However, there may be instances when a mAb undergoes significant elimination in peripheral tissues (e.g., via receptor-mediated endocytosis). In these cases, the aforementioned methods of analysis can lead to errors in estimating $\mathrm{V}_{\mathrm{ss}}(63,65)$. Therefore, volumes of distribution reported in the literature for mAbs should be interpreted with caution.

\section{Antibody Clearance}

The two general pathways by which drugs are eliminated from the body are metabolism (e.g., catabolism) and excretion. However, the role of non-catabolic routes, such as renal and biliary excretion, in the clearance of $\mathrm{mAbs}$ is negligible. The glomerular filtration of mAbs is largely limited by their size, as the molecular cut-off weight for filtration is approximately $70 \mathrm{kDa}(66)$. While biliary excretion has been reported for IgA, it does not appear to be a significant route for elimination of $\operatorname{IgG}(9$, 67). The primary route by which antibodies are eliminated is cellular uptake followed by proteolytic degradation. Therapeutic antibodies often exhibit two distinct catabolic pathways (8): 1) a non-specific, linear (first-order) clearance pathway mediated by interaction between the Fc region of the antibody and Fc receptors (i.e., FcRn and Fc $\gamma$ receptors) and 2) a nonlinear (target-mediated) clearance pathway mediated by the specific interaction between the Fab region of the antibody and its pharmacologic target.

\section{Fc-Receptor-Mediated Elimination}

The previous use of non-specific with regards to the linear clearance pathway reflects the notion that Fc-mediated elimination is a common pathway shared by both endogenous IgG and therapeutic IgG mAbs with a functional human Fc domain. Virtually all cells in the body have the ability to take up proteins and other macromolecules from the surrounding fluid space via endocytosis (e.g., fluid-phase pinocytosis). The formed endosome may subsequently be delivered to a lysosome, where its contents undergo enzymatic degradation. However, unlike most other proteins in the 
endosome, a significant portion of IgG can be salvaged from lysosomal catabolism by an FcRn-mediated mechanism in cells expressing FcRn (68-70).

Following cellular uptake of $\operatorname{IgG}$, the environment inside the early endosome becomes mildly acidic. IgG binds to FcRn in a $\mathrm{pH}$ dependent manner, and the decrease in $\mathrm{pH}$ allows IgG to bind to FcRn expressed within the endosome. IgG that is bound to FcRn is redirected to the cell surface, while unbound IgG is delivered to the lysosome for degradation. This partitioning of IgG-FcRn complexes and unbound IgG into two different pathways appears to occur within the sorting endosome (70). Once the IgGFcRn complex is transported to the cell surface, the affinity of IgG to FcRn decreases as the result of the physiological $\mathrm{pH}$, and $\mathrm{IgG}$ is released into the extracellular fluid. This salvage pathway helps to explain the much longer elimination half-life of $\operatorname{IgG}(\sim 21$ days) compared to that of the other immunoglobulin classes whose half-lives range from 2-10 days (6). While the IgG1, IgG2, and IgG4 subclasses exhibit a half-life of around 21 days, the half-life for IgG3 is only 7 days. The shorter half-life of IgG3 has been attributed to binding differences to FcRn compared to the other IgG subclasses (8). As the number of FcRn receptors is limited, the salvage pathway can be expected to be capacity limited. This is supported by findings from a study by Morell et al., in which shorter IgG half-lives were observed in patients with elevated serum IgG concentrations compared to those with normal IgG levels (71). However, the doses commonly administered for therapeutic mAbs are not likely to cause significant enough increases in serum IgG concentrations that result in saturation of the FcRn receptor (72). Therapeutic $\mathrm{mAbs}$ are commonly administered at doses $<10 \mathrm{mg} / \mathrm{kg}$, which would increase the total body IgG by $<1-2 \%$, as humans typically possess $50-100 \mathrm{~g}$ of endogenous $\operatorname{IgG}(9)$. Furthermore, as will be discussed later in the review, the estimates for total clearance in mAbs with linear elimination characteristics and for the clearance of the linear elimination pathway in mAbs with parallel linear and nonlinear elimination were quite similar to the clearance of endogenous IgG. The FcRn receptor is expressed in a wide variety of cells and tissues throughout the body including the vascular endothelium, monocytes, macrophages, dendritic cells, hepatocytes, and epithelial cells of the intestine, renal proximal convoluted tubules and upper airways $(73,74)$.

Another set of receptors that bind to the Fc portion of IgG are the Fc $\gamma$ receptors. These receptors play a significant role in mediating various immune effector functions such as phagocytosis and antibody dependent cellular cytotoxicity (75). There are three different classes of Fc $\gamma$ receptors (i.e., Fc $\gamma$ RI, II, III) and they differ with regards to their cellular distribution, specificity for the different IgG subclasses, and affinity for monomeric or immune-complexed IgG (10). The Fc $\gamma$ receptors are expressed on a wide variety of cells including monocytes, macrophages, dendritic cells, neutrophils, NK (natural killer) cells, B cells, and hepatocytes $(10,73)$. Based on their innate roles in immune responses, Fc $\gamma$ receptors may be involved in the clearance of soluble mAbantigen immune complexes or cells opsonized by the mAb. However, the significance of $\mathrm{Fc} \gamma$ receptor-mediated catabolism of mAbs is not as well understood as the role of FcRn. 
The interaction between a mAb and its pharmacologic target can contribute to the elimination of the mAb. A concept often applied to the PK of mAbs is target-mediated drug disposition in which the interaction between a drug and pharmacologic target influences its PK characteristics $(76,77)$. Receptor-mediated endocytosis, an example of target-mediated drug disposition, involves binding of the $\mathrm{mAb}$ to a receptor on the cell surface, which triggers internalization and subsequent lysosomal degradation of the mAbreceptor complex. The number of receptors within the distribution space of the $\mathrm{mAb}$ is limited, and therefore, the receptors may become saturated at therapeutic doses of the $\mathrm{mAb}$ resulting in nonlinear clearance. The nonlinear clearance observed with many $\mathrm{mAbs}$ is believed to be due to saturation of target-mediated clearance pathways (8). The role that target-mediated clearance plays in the total clearance of the $\mathrm{mAb}$ is dependent on a number of factors including the concentration and distribution of the receptor, and rates of receptor internalization and turnover (8).

Therapeutic mAbs that bind to soluble antigens may also undergo target-mediated elimination. One potential mechanism that has been suggested is in which the soluble mAb-antigen complex binds to Fc $\gamma$ receptors on cells such as monocytes and macrophages, which subsequently triggers internalization and catabolism of the complex (9). If this elimination mechanism was present for a given therapeutic $m A b$, then it would be reasonable to anticipate that the clearance of the soluble mAb-antigen complex would be greater compared to that for the unbound and free $\mathrm{mAb}$. In a population $\mathrm{PK} / \mathrm{PD}$ analysis of omalizumab (an anti-IgE $\mathrm{mAb}$ ), the apparent clearance of the omalizumabIgE complex was $0.32 \mathrm{~L} /$ day compared to $0.18 \mathrm{~L}$ /day for free omalizumab (41). The nonlinear PK observed for mAbs, such as omalizumab and denosumab $(24,78,79)$, which target soluble antigens, suggests that saturable target-mediated mechanisms of elimination are not only limited to mAbs with membrane bound antigens (9).

\section{Clearance Models}

The different PK models used in the population analyses are listed in Table 1-1. In most studies compartmental PK models with linear, nonlinear, or parallel linear and nonlinear elimination pathways were used to describe the PK of the mAb. In the population PK/PD analyses of efalizumab (35) and omalizumab (41) more complex mechanistically based target-mediated elimination models were applied. A commonly used PK model that can be related to the distinct physiologic elimination pathways is a two-compartment model with parallel linear and nonlinear elimination from the central compartment, which is depicted in Figure 1-2A. In this PK model, the total clearance $\left(\mathrm{CL}_{\mathrm{T}}\right)$ of the $\mathrm{mAb}$ is comprised of clearance from the nonlinear pathway $\left(\mathrm{CL}_{\mathrm{NL}}\right)$ plus that of the linear pathway $\left(\mathrm{CL}_{\mathrm{L}}\right)$. Physiologically, the nonlinear pathway is thought to be related to elimination of the $\mathrm{mAb}$ via saturable target-mediated mechanisms (e.g., receptor-mediated endocytosis), while the linear component represents elimination pathways not saturable at therapeutic mAb concentrations such as Fc-mediated elimination. The relationship between the clearance components of the model $\left(\mathrm{CL}_{\mathrm{L}}\right.$, 
Figure 1-2. Pharmacokinetic behavior of a monoclonal antibody with parallel linear and nonlinear elimination pathways. Schematic of a representative structural pharmacokinetic model with parallel elimination pathways (A). Plot of clearance dependence on serum concentrations (B). Simulated serum concentration-time profiles (C) using the pharmacokinetic model in (A) after single IV bolus doses of 50, 100, 200, 400,800 , and $1600 \mathrm{mg}$. Parameter values of $18 \mathrm{mg} /$ day $\left(\mathrm{V}_{\max }\right), 5 \mathrm{mg} / \mathrm{L}\left(\mathrm{K}_{\mathrm{m}}\right), 0.4 \mathrm{~L} /$ day $\left(\mathrm{CL}_{\mathrm{L}}\right), 4 \mathrm{~L}\left(\mathrm{~V}_{1}\right), 3 \mathrm{~L}\left(\mathrm{~V}_{2}\right)$, and 1.2 L/day $(\mathrm{Q})$ were used for the simulation as well as for the clearance versus concentration plot. $\mathbf{C}_{\mathbf{1}}=$ concentration in central compartment; $\mathbf{C L}_{\mathbf{L}}$ $=$ clearance of the linear elimination pathway; $\mathbf{C} \mathbf{L}_{\mathbf{N L}}=$ clearance of the nonlinear elimination pathway (calculated as $\mathrm{V}_{\max } /\left(\mathrm{K}_{\mathrm{m}}+\mathrm{C}_{1}\right)$ ); $\mathbf{C} \mathbf{L}_{\mathbf{T}}=$ total clearance (calculated as $\left.\mathrm{CL}_{\mathrm{NL}}+\mathrm{CL}_{\mathrm{L}}\right) ; \mathbf{K}_{\mathbf{m}}=$ concentration at which elimination is half maximum; $\mathbf{Q}=$ intercompartmental clearance; $\mathbf{V}_{\mathbf{1}}=$ volume of central compartment; $\mathbf{V}_{\mathbf{2}}=$ volume of peripheral compartment; $\mathbf{V}_{\mathbf{m a x}}=$ maximum elimination rate. 


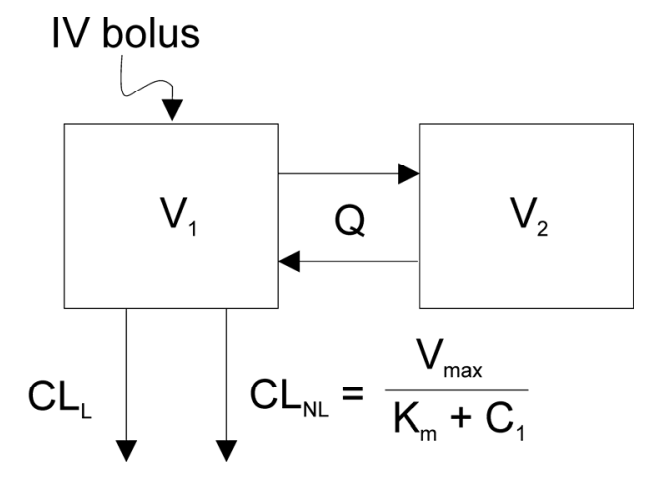

A

B

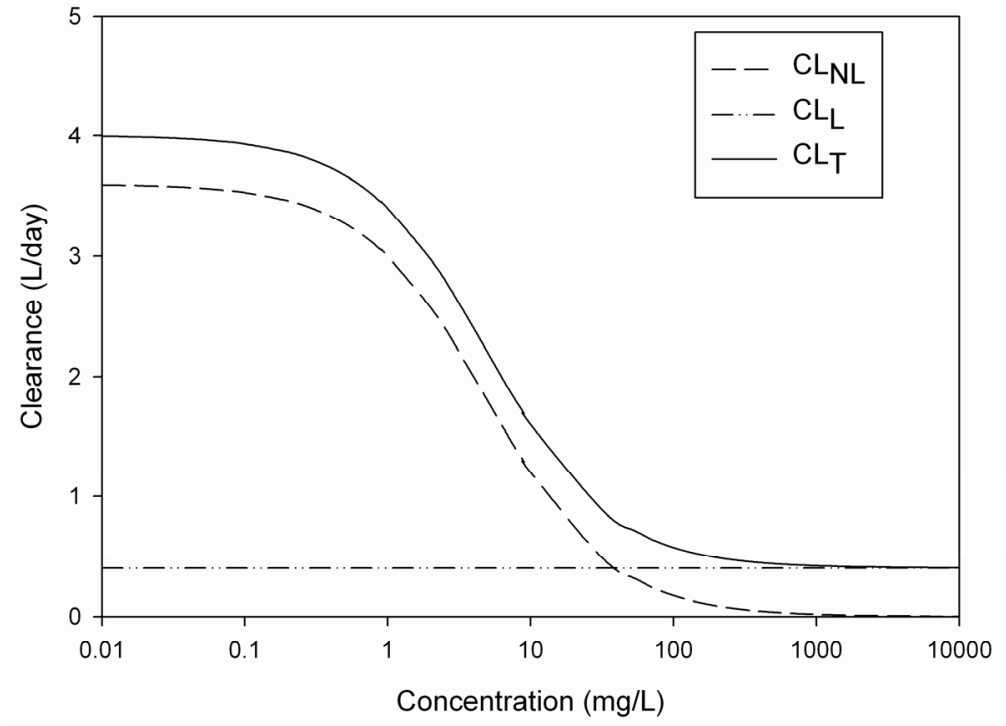

C

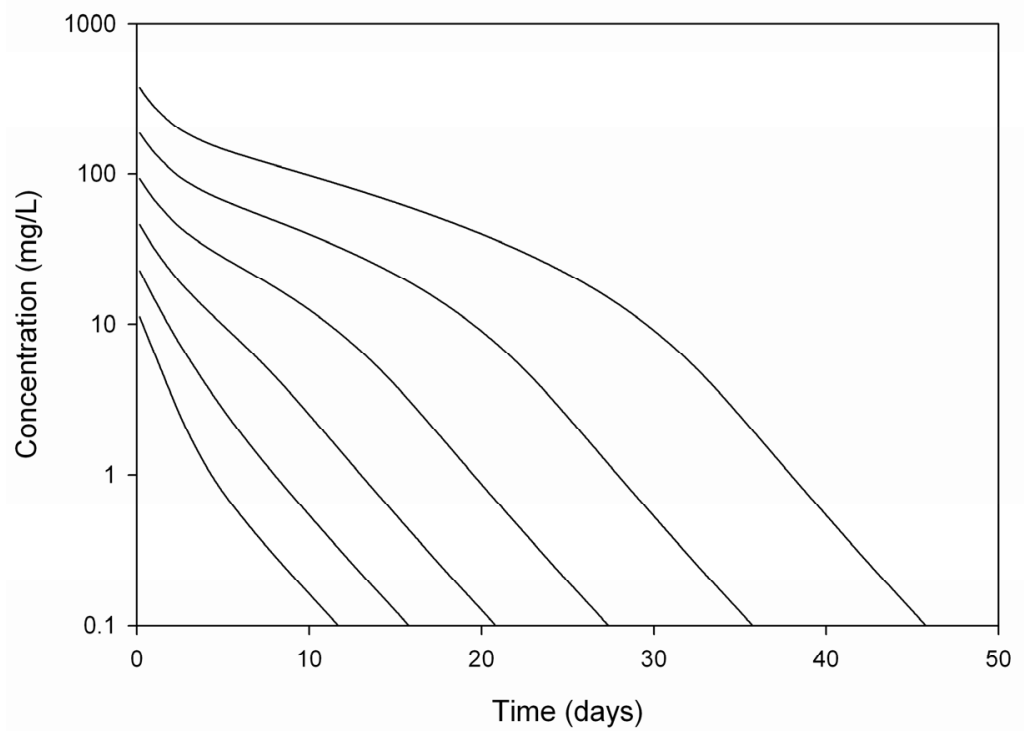


$\mathrm{CL}_{\mathrm{NL}}$, and $\mathrm{CL}_{\mathrm{T}}$ ) and $\mathrm{mAb}$ concentration is described in Figure 1-2B. At low concentrations total clearance is relatively unaffected by the mAb concentration, and target-mediated elimination contributes a significant portion to the overall clearance of the $\mathrm{mAb}\left(\mathrm{CL}_{\mathrm{NL}}\right.$ is approximately 7 times larger than $\left.\mathrm{CL}_{\mathrm{L}}\right)$. With increasing concentrations, total clearance decreases dramatically as the target-mediated elimination pathway starts to become saturated. The concentration range at which this significant decrease in total clearance occurs is dependent on the $\mathrm{Kd}$ (dissociation constant) and/or EC50 of the mAb-receptor interaction, and lies for many mAbs in the range of 0.1-100 $\mathrm{mg} / \mathrm{L}$. At high concentrations, the total clearance approaches that of the first-order process $\left(\mathrm{CL}_{\mathrm{L}}\right)$, while the contribution from the nonlinear pathway becomes negligible. A biphasic clearance versus dose profile has been observed for several therapeutic mAbs including cetuximab, trastuzuamb, and efalizumab (35, 79-82). Another characteristic of this model is an S-shaped curve in the concentration-time profile (Figure 1-2C). This may be observed at higher doses if concentrations are sampled for a prolonged time period, and is due to an increase in total clearance coinciding with an increase in CL $_{\mathrm{NL}}$ as concentrations decrease. Given a two-compartment PK model with one linear and one nonlinear elimination pathway, there are different structural variations that can be conceived by varying the location (i.e., central or peripheral compartment) of the two elimination pathways. Issues regarding identifiability and indistinguishability of these different models has been investigated by Godfrey and colleagues (83). In most studies that used a parallel elimination model, the configuration was one where both the linear and nonlinear elimination pathways originated from the central compartment. However, other structural variations were evaluated in the population PK analyses of matuzumab and CP-751, $971(31,40)$.

The most frequent PK model used in the population analyses was a one- or twocompartment model with linear elimination (Table 1-1), which is inconsistent with the observation that many therapeutic mAbs exhibit nonlinear clearance $(6,8)$. One reason for this discrepancy may have been related to the doses (concentrations) studied in the population PK analysis. Referring back to Figure 1-2B, the dose of the mAb may result in concentrations that are high enough to saturate the target-mediated elimination pathway. A compartmental model with linear elimination may adequately describe the data at these high concentrations, as the nonsaturable Fc-mediated elimination pathway becomes the major determinant of $\mathrm{mAb}$ clearance. Nonlinear clearance has been observed for efalizumab and trastuzumab, and this was attributed to saturation of targetmediated clearance pathways via their respective targets, CD11a and HER-2 (33, 35, 79, 82). Contrary to these observations, two separate population PK analyses of efalizumab and trastuzumab found that their PK was adequately described using a compartmental model with linear elimination $(33,46)$. Based on PK data from dose escalation studies of efalizumab (35) and trastuzumab (82), the doses of these mAbs investigated in the aforementioned population PK analyses may have resulted in steady-state concentrations where target-mediated clearance was saturated and thus the use of a PK model with linear elimination was warranted. In a population PK analysis of bevacizumab in patients with solid tumors, concentration data was pooled from a number of different clinical trials where the dosing regimen for bevacizumab varied (28). The data from 10 patients that received bevacizumab doses of 0.1 and $0.3 \mathrm{mg} / \mathrm{kg}$ were excluded from the analysis as 
clearance at these doses was faster, and these doses were not evaluated in further clinical trials. The excluded set of patients represented a small fraction of the 491 patients included in the population PK study. The final population PK model for bevacizumab included a two-compartment model with linear elimination (28). The use of a PK model with linear elimination rather than with nonlinear elimination seems reasonable in this case, since the population PK model was used to describe bevacizumab concentrations at clinically relevant doses which did not included the doses of 0.1 and $0.3 \mathrm{mg} / \mathrm{kg}$ excluded from the analysis. The lowest dose group was also excluded in a population PK analysis of golimumab, an antibody against tumor necrosis factor alpha (TNF- $\alpha$ ), in patients with rheumatoid arthritis (34). The dataset used to build the population PK model included 21 patients who received single IV doses of golimumab ranging from $0.3-10 \mathrm{mg} / \mathrm{kg}$, while 3 patients who received $0.1 \mathrm{mg} / \mathrm{kg}$ were excluded from the analysis. The inclusion of data from the $0.1 \mathrm{mg} / \mathrm{kg}$ group resulted in convergence difficulties, and it was believed that this may be due to a significant influence of TNF- $\alpha$ mediated disposition at a very low dose. The final population PK model for golimumab included a two-compartment model with linear elimination (34).

\section{Clearance Population Estimates}

Population estimates and their between-subject variability for clearance related PK parameters are summarized in Table 1-4. The estimates for the maximum elimination rate $\left(\mathrm{V}_{\max }\right)$ and the $\mathrm{mAb}$ concentration at which elimination is at half maximum $\left(\mathrm{K}_{\mathrm{m}}\right)$ varied considerably among the different therapeutic mAbs. As nonlinear clearance is often attributed to target-mediated mechanisms, it can be expected that $\mathrm{V}_{\max }$ and $\mathrm{K}_{\mathrm{m}}$ will be specific for a given $\mathrm{mAb}$ and its pharmacologic target. The Michaelis-Menten type parameters $\left(\mathrm{V}_{\max }\right.$ and $\left.\mathrm{K}_{\mathrm{m}}\right)$ can be related to parameters of a full model of target-mediated drug disposition $(84,85)$. While between-subject variability was always estimated for $\mathrm{V}_{\max }$, it was often not estimated for $\mathrm{K}_{\mathrm{m}}$ thereby treating it as a fixed value across individuals. Given that $\mathrm{V}_{\max }$ is related to the number of pharmacologic targets within the $\mathrm{mAb}$ distribution space and $\mathrm{K}_{\mathrm{m}}$ is related to the affinity of the mAb to its target, it is a reasonable approach to hypothesize that physiologically there is more variability in $V_{\max }$ among individuals than in $\mathrm{K}_{\mathrm{m}}$. Between-subject variability in $\mathrm{V}_{\max }$ was typically moderate to high with a median (range) value of $34 \% \mathrm{CV}(15-65 \% \mathrm{CV})$ across the studies. When estimated, variability in $\mathrm{K}_{\mathrm{m}}$ tended to be quite large with most estimates being greater than $100 \% \mathrm{CV}$. For mAbs with population estimates of $\mathrm{V}_{\max }$ and $\mathrm{K}_{\mathrm{m}}$, their intrinsic clearance $\left(\mathrm{CL}_{\text {int }}\right)$ was calculated as $\mathrm{V}_{\max } / \mathrm{K}_{\mathrm{m}}$ and is reported in Table 1-4. All of the therapeutic mAbs in Table 1-4, for which $\mathrm{CL}_{\text {int }}$ was calculated, bind to a cell surface antigen. The pharmacologic target for denosumab, receptor activator of nuclear factor$\kappa \mathrm{B}$ ligand (RANKL), is expressed not only in a membrane-bound form but also in a soluble form $(86,87)$. An interesting observation was made regarding the relationship between $\mathrm{CL}_{\text {int }}$ and the location (i.e., blood or tissue) of the cell membrane target. Sibrotuzumab, matuzumab, cetuximab, denosumab, and CP-751,871 all bind to cell membrane antigens primarily expressed in tissue, and their calculated $\mathrm{CL}_{\text {int }}$ values ranged from 0.47-3.70 L/day. On the other hand, efalizumab, alemtuzumab, and clenoliximab, target different $\mathrm{CD}$ antigens largely expressed on white blood cells, and their $\mathrm{CL}_{\text {int }}$ was 
much larger with values ranging from 27.8-57.0 L/day. Mechanistically, the larger $\mathrm{CL}_{\text {int }}$ observed for mAbs that target blood cells may be due to a larger number of antigens within their primary distribution space compared to mAbs with membrane bound targets in tissue. Panitumumab, cetuximab, and matuzumab are all anti-EGFR (epidermal growth factor receptor) $\mathrm{mAbs}$, but the $\mathrm{CL}_{\text {int }}$ for panitumumab $(28.4 \mathrm{~L} /$ day $)$ was much larger compared to that for cetuximab $(1.42 \mathrm{~L} /$ day) and matuzumab $(2.73 \mathrm{~L} /$ day) (Table 1-4). It is uncertain as to why these differences in $\mathrm{CL}_{\text {int }}$ exist between the anti-EGFR $\mathrm{mAbs}$, but one could hypothesize that the larger $\mathrm{CL}_{\text {int }}$ observed for panitumumab is in part due to its higher affinity for EGFR $\left(\mathrm{K}_{d}=5 \times 10^{-11} \mathrm{M}\right)$ compared to cetuximab $\left(\mathrm{K}_{\mathrm{d}}=39\right.$ x $\left.10^{-11} \mathrm{M}\right)$ and matuzumab $\left(\mathrm{K}_{\mathrm{d}}=34 \times 10^{-11} \mathrm{M}\right)(88-90)$.

Assuming a volume of distribution of $6.2 \mathrm{~L}$ and an elimination half-life of 21 days for IgG (64) (with the exception of the IgG3 subclass), the clearance for endogenous IgG is approximately $0.21 \mathrm{~L} /$ day. The population estimates for $\mathrm{CL}_{\mathrm{L}}$ were relatively close to the clearance of endogenous $\mathrm{IgG}$, as the median (range) $\mathrm{CL}_{\mathrm{L}}$ estimate was $0.31 \mathrm{~L} /$ day (0.066-0.535 L/day). The discrepancy between $\mathrm{CL}_{\mathrm{L}}$ values and the clearance for endogenous IgG may be due in part to different affinities of the therapeutic mAbs to the FcRn receptor and Fc $\gamma$ receptor subtypes compared to IgG. The population clearance estimates for several of the mAbs were around $0.2 \mathrm{~L} /$ day, and thus quite close to the clearance for endogenous IgG. This suggests that either these mAbs do no exhibit saturable target-mediated clearance or that clearance via this pathway was negligible at the concentrations studied in the population analysis.

The population clearance estimates for inolimomab, mAbF19, HuCC49 $4 \mathrm{CH} 2$, and basiliximab, were much larger than those for other mAbs. In the case of the first three $\mathrm{mAbs}$ listed, their increased clearance may have been due to impaired binding to the FcRn receptor, which protects IgG from proteolytic degradation (55). Both inolimomab and $\mathrm{mAbF} 19$ are murine $\operatorname{IgG} \mathrm{mAbs}(38,39)$. The rapid elimination of murine mAbs in humans has been attributed to the lack of binding of murine IgG to the human FcRn receptor (91). No indication was given in the population PK analyses of inolimomab and $\mathrm{mAbF} 19$ if antibodies against these therapeutic mAbs were measured and/or detected $(38,39)$. Considering that inolimomab and $\mathrm{mAbF} 19$ are murine $\mathrm{mAbs}$, an immune response and formation of antibodies against the mAbs could be another potential reason for the larger clearance estimates compared to other therapeutic mAbs. $\mathrm{HuCC} 49 \triangle \mathrm{CH} 2$ is a humanized IgG1 mAb, but is different in that it has a deletion in the constant heavy 2 domain to minimize immunogenicity (17). The authors suggested that the increased clearance in HuCC49 $4 \mathrm{CH} 2$ may have been due to the constant heavy 2 domain deletion, which could have affected binding to the FcRn receptor (17). This appears to be a valid argument as the FcRn receptor binds to a site on IgG formed between the constant heavy 2 and constant heavy 3 domains (10).

The population PK of basiliximab has been investigated in patients with kidney (26) and liver (27) transplants, and the clearance in liver recipients was found to be larger compared to kidney receipients. One likely explanation for the larger clearance in liver transplant patients is the additional route of basiliximab clearance through drained ascites fluid $(27,92)$. 
Apparent clearance $(\mathrm{CL} / \mathrm{F})$ was estimated in the population $\mathrm{PK}$ analyses of omalizumab, efalizumab, and ustekinumab (Table 1-4), which have an F following SC dosing of around $62 \%, 50 \%$, and $57 \%$, respectively $(33,41,47)$. After adjusting for $\mathrm{F}$, the clearance of omalizumab, efalizumab, and ustekinumab, are approximately 0.11 , 0.65 , and $0.27 \mathrm{~L} /$ day, respectively. The between-subject variability in $\mathrm{CL}_{\mathrm{L}}$ and $\mathrm{CL}_{\mathrm{T}}$, much like the variability in $\mathrm{V}_{\max }$, was moderate to large with estimates ranging from 20 $59 \% \mathrm{CV}$.

\section{Covariates}

Identified covariates in population analyses of therapeutic mAbs and their estimated effects on PK parameters are presented in Table 1-5. Covariates were commonly identified for the PK parameters $\mathrm{V}_{\max }, \mathrm{CL}_{\mathrm{L}}, \mathrm{CL}_{\mathrm{T}}$, and $\mathrm{V}_{1}$. No covariates were found to influence $\mathrm{K}_{\mathrm{m}}$, which is not unexpected. As $\mathrm{K}_{\mathrm{m}}$ reflects the intrinsic affinity of the $\mathrm{mAb}$ to its target, commonly evaluated covariates such as those related to demographics, size measurements, and blood chemistry/hematology labs would not be expected to have an effect on $\mathrm{K}_{\mathrm{m}}$. Polymorphic expression of the target could theoretically have an effect on the $\mathrm{K}_{\mathrm{m}}$, but this type of genetic information has not been incorporated into any population PK models published to date for therapeutic mAbs. Polymorphic expression in the gene that encodes the Fc $\gamma$ IIIa receptor (FCGR3A) has been associated with better clinical and/or biological response in non-Hodgkin's lymphoma patients treated with rituximab $(93,94)$, Crohn's disease patients treated with infliximab (95), and patients with breast cancer receiving trastuzumab therapy (96).

\section{Demographic and Anthropometric Measurements}

Size covariates are probably the most frequently identified and clinically relevant covariates in population PK analyses, and are well established as predictors of systemic exposure for small-molecule drugs as well as therapeutic mAbs (13). Based on the findings summarized in Table 1-5, measures of body size were clearly the most commonly identified covariates found to influence the PK of therapeutic mAbs. This is not surprising since PK parameters such as clearance and volume are often functions of body size (97). Measures of body size that were frequently evaluated as covariates include body weight (WGT), ideal body weight (IBW), and body surface area (BSA). Common practice in the $\mathrm{mAb}$ population $\mathrm{PK}$ analyses was to evaluate different size covariates during the covariate modeling process to identify which, if any, were the most influential and should remain in the model. Of the different measures of body size, WGT was the most common size covariate included in the final population PK model (Table 1-5). In most studies, the effect of body size on a PK parameter was modeled using a power function and the exponent in the function was treated as an estimable parameter. However, in a population PK analysis of HuCC49 $\triangle \mathrm{CH} 2$ in colorectal cancer patients, the exponent was fixed to commonly used allometirc values of 0.75 for clearance terms and 1.0 for volume terms (17). 
The use of fixed dosing versus body size-based dosing for therapeutic mAbs in adults was recently addressed by Wang et al. (98). A simulation study was carried out using published population PK and/or PD models for 12 therapeutic mAbs, and the performance of the two dosing strategies in reducing PK and/or PD variability was investigated. The two dosing approaches performed similarly across the $12 \mathrm{mAbs}$ investigated with fixed dosing being better for some mAbs and body size-based dosing for others. Based on the findings, the authors recommended using fixed dosing in firstin-human studies, and once sufficient data is available to assess the effect of body size on the $\mathrm{PK} / \mathrm{PD}$ of the $\mathrm{mAb}$, these findings can be used in part to determine the appropriate dosing strategy for subsequent phase 3 studies (98).

Other demographic variables besides body size that were often evaluated as covariates included age, gender, and race. Even after adjusting for body size, gender was identified as a predictor of $\mathrm{CL}_{\mathrm{L}}, \mathrm{CL}_{\mathrm{T}}$, and $\mathrm{V}_{1}$ in a small number of studies (Table 1-5). The effect of gender on the PK of ustekinumab and infliximab was relatively small and not considered to be clinically relevant $(36,47)$. In the population PK analyses of bevacizumab and rituximab, the evaluation of gender as a covariate found that males had a larger $\mathrm{CL}_{\mathrm{T}}$ and $\mathrm{V}_{1}$ (values ranged from 17\%-39\% larger) $(28,44)$. However, based on the prescribing information for these two $\mathrm{mAbs}$, no dose adjustments for gender are recommended based on similar efficacy and safety profiles in males and females (99, $100)$. Race was evaluated as a covariate in a number of population PK studies including bevacizumab (28), cetuximab (29), pertuzumab (43), and alemtuzumab (23), but was not found to influence the PK of any of these mAbs. The percentage of non-Caucasian patients was small in many studies, and may have precluded the ability to identify any possible differences between race groups. In population PK studies of infliximab (36) and golimumab (34) race was not investigated as a covariate because most patients were Caucasian. The only study where race was identified as a covariate was a population PK analysis of ustekinumab in psoriasis patients (47). The $\mathrm{V}_{1} / \mathrm{F}$ for ustekinumab was found to be $11.1 \%$ lower in non-Caucasian patients versus that for Caucasians, but this was not considered to be clinically relevant. Age was identified as a covariate in only the population PK analyses of efalizumab and panitumumab $(33,42)$. Age was found to have an opposite effect in the two studies, as age was positively correlated with $\mathrm{CL} / \mathrm{F}$ in the efalizumab analysis, and in the panitumumab analysis age was negatively correlated with $\mathrm{V}_{\max }$.

\section{Receptor Number and Disease-Related Factors}

As many therapeutic mAbs undergo receptor-mediated endocytosis following binding to their target, it can be expected that the number of receptors within the distribution space of the mAb would affect its clearance. Covariates related to receptor number may include flow cytometry measurements (e.g., receptor density and receptorpositive cell counts) and analysis of biopsy samples using immunohistochemistry or fluorescence in situ hybridization. Patient status and disease severity could also be related to receptor number, as one could hypothesize that for some conditions a patient 
with more severe disease would have a higher receptor expression. Time or duration of treatment is another variable that may be related to receptor number if administration of the therapeutic $\mathrm{mAb}$ results in down modulation of its target over time.

Omalizumab and efalizumab are excellent examples where the effect of targetmediated elimination was accounted for using a more complex mechanistic-based population PK/PD model $(35,41)$. Similarly, a population PK/PD analysis in patients with B-cell chronic lymphocytic leukemia found that alemtuzumab exhibited nonlinear clearance attributable to saturation of target-mediated clearance mechanisms (23). The only covariate found to influence the PK of alemtuzumab was white blood cell (WBC) count, which was a strong positive predictor of $\mathrm{V}_{\max }$. This could be expected as alemtuzumab targets CD52, an antigen present on the cell surface of WBCs including lymphocytes and monocytes. The WBC count for each patient was updated over time when possible, and WBC counts were shown to generally decrease following repeated administration of alemtuzumab. Therefore, alemtuzumab exhibited both time- and concentration-dependent clearance (23). An interesting use of time as a covariate was applied in a population PK analysis of rituximab in patients with non-Hodgkin's lymphoma (101). The authors hypothesized that an observed increase in rituximab halflife over time was due to a decrease in clearance as B cell (CD19+) count and/or tumor burden also decreased. The PK of rituximab was described using a two-compartment model with two clearance pathways, where the clearance of one pathway remained constant while the other clearance decreased over time as a first-order process. A covariate analysis found that patients with higher CD19 counts or larger measurable tumor lesions at baseline had a larger initial time-varying clearance (101).

Trastuzumab is a humanized IgG1 $\mathrm{mAb}$ that targets the human epidermal growth factor receptor 2 (HER2). The extracellular domain (ECD) of HER2 can be shed from the cell surface into the systemic circulation (soluble antigen), thereby providing an additional site for trastuzumab binding (46). In a population PK analysis of trastuzumab in patients with HER2 positive metastatic breast cancer, several variables were investigated as potential covariates including HER 2 overexpression by immunohistochemistry (IHC), number of metastatic sites, and serum concentrations of the ECD of HER2 (46). All of the covariates were baseline values, hence no timevarying covariates were used. The number of metastatic sites was identified as the most influential covariate on clearance, which was $22 \%$ higher in patients with four or more sites. The baseline concentration of ECD was found to positively influence both $\mathrm{CL}_{\mathrm{T}}$ and $\mathrm{V}_{1}$. Patients with ECD concentrations of $200 \mathrm{ng} / \mathrm{ml}$ or greater had a $14 \%$ and $40 \%$ larger $\mathrm{CL}_{\mathrm{T}}$ and $\mathrm{V}_{1}$, respectively, compared to patients with a median ECD concentration of 8.23 $\mathrm{ng} / \mathrm{ml}$. The impact of ECD levels on the $\mathrm{CL}_{\mathrm{T}}$ of trastuzumab is in line with nonclinical studies that suggested that the trastuzumab-ECD complex has a higher clearance than unbound trastuzumab (46). Potential reasons why ECD had an effect on $V_{1}$ were not addressed in the paper. HER2 overexpression based on IHC was not retained in the final model as a significant covariate.

According to the prescribing information for bevacizumab, a population PK analysis found that patients with a high tumor burden (at or above the median value of 
tumor surface area) had a clearance of $0.249 \mathrm{~L}$ /day versus $0.199 \mathrm{~L} /$ day in patients with tumor burdens below the median (99). Tumor burden could not be evaluated as a covariate in a recently published population PK analysis of bevacizumab, because data was not available in all of the clinical trials included in the analysis (28). However, albumin and alkaline phosphatase were found to positively influence the clearance of bevacizumab, and both were correlated with tumor burden in this study. Assessments of physical performance and disease severity using scoring systems such as the Karnofsky performance scale, Psoriasis Area and Severity index (PASI), and Bath Ankylosing Spondylitis Disease Activity index, are commonly evaluated as covariates in population PK studies of mAbs used in areas of oncology and immunology $(29,36,40,46)$. The only study to identify one of these scoring systems as a covariate was a population PK analysis of efalizumab, where PASI was found to have a modest effect on CL/F (33).

\section{Pharmacokinetic Drug Interactions}

Pharmacokinetic drug-drug interactions between therapeutic mAbs and conventional small-molecule drugs are usually not expected, since mAbs are presumably not substrates for cytochrome P-450 enzymes or drug transporters such as P-glycoprotein. As such, many of the clinical PK drug interaction studies that have been conducted between therapeutic mAbs and small-molecule drugs did not identify any interaction (102). However, there have been a small number of clinical studies where smallmolecule drugs were found to have an effect on the PK of the mAb or vice versa (102). Since therapeutic mAbs are often administered with other treatments, concomitant medication was evaluated as a potential covariate in several of the population PK analyses. In all but one of the studies, concomitant medication was not found to influence the PK of the mAb. Examples of mAbs where concomitant medication was not identified as a covariate in the population PK analysis include cetuximab (29), rituximab (44), trastuzumab (46), and infliximab (36). Concomitant chemotherapy was identified as a covariate in a population PK analysis of bevacizumab (28). The clearance of bevacizumab when administered with the bolus-IFL (irinotecan/5fluorouracil/leucovorin) regimen was $17 \%$ lower compared to all other concomitant chemotherapy regimens combined, however, bevacizumab clearance when administered as monotherapy did not differ from that when administered with the bolus-IFL regimen. The effect of the concomitant chemotherapy on the clearance of bevacizumab was minimal and does not likely necessitate any dosage adjustments.

\section{Dosage Level}

A therapeutic mAb may be known to exhibit nonlinear clearance based on prior PK studies, but the concentration data available in a current population PK analysis may not support a more complex PK model with nonlinear clearance. However, if concentration data in the population analysis is available from multiple dose groups, the nonlinear clearance of the $\mathrm{mAb}$ could be accounted for in part by evaluating dose as a predictor of clearance. Such an approach was taken in a population PK analysis of 
efalizumab in psoriasis patients who received weekly SC doses of $1 \mathrm{mg} / \mathrm{kg}$ or $2 \mathrm{mg} / \mathrm{kg}$ (33). Efalizumab has been shown to exhibit nonlinear clearance attributable to saturation of target-mediated elimination mechanisms (35), and accordingly, various onecompartment models with linear, nonlinear, and parallel linear and nonlinear elimination pathways were evaluated in the study (33). A one-compartment model with linear elimination was found to adequately describe the PK of efalizumab, as the limited concentration data did not support the PK models with nonlinear elimination. Dose was investigated as a covariate on $\mathrm{CL} / \mathrm{F}$, and it was found that patients in the $2 \mathrm{mg} / \mathrm{kg}$ group had a $24 \%$ lower CL/F versus patients in the $1 \mathrm{mg} / \mathrm{kg}$ group. Dose group was also found to be a predictor of clearance in a population analysis of the mAb ING-1 in patients with advanced adenocarcinomas (103). The PK of ING-1 was investigated across four IV dose levels ranging from 0.03 to $1.0 \mathrm{mg} / \mathrm{kg}$. The clearance of ING-1 was noted to decrease as the dose was increased, but the data did not support a PK model with nonlinear elimination. Therefore, a one-compartment model with linear elimination was selected with dose included as a covariate on clearance. The clearance of ING-1 decreased from 56.7 to $21.8 \mathrm{~mL} / \mathrm{kg}$ /day in the $0.03 \mathrm{mg} / \mathrm{kg}$ and $1.0 \mathrm{mg} / \mathrm{kg}$ dose groups, respectively.

For a therapeutic $\mathrm{mAb}$ that exhibits nonlinear PK due to saturable target-mediated mechanisms, the ability to detect and adequately characterize the nonlinearity in a population analysis is largely dependent on the availability of concentration-time data from a wide range of doses. However, as often encountered in drug development, concentration-time data may only be available from clinical studies where the doses studied (which may be clinically and therapeutically relevant) are high enough to saturate the nonlinear target-mediated elimination of the therapeutic $\mathrm{mAb}$, thereby making it difficult to detect and characterize the nonlinear PK. In such a case, if the population PK model is to be used for describing/predicting concentrations at doses where the nonlinear elimination pathway is at or near saturation, then using a PK model with linear elimination would be a reasonable approach as the Fc-mediated elimination pathway becomes the major determinant of the mAb clearance (see Figure 1-2B).

\section{Immunogenicity}

As therapeutic mAbs are exogenous proteins, they can elicit an immune response resulting in the formation of endogenous antibodies against the mAb. The immunogenicity of a therapeutic mAb can be influenced by several factors including the structure and murine content of the antibody, immune status of the patient (e.g., immunocompetent or immunosuppressed), dose level, dosing frequency, and dosing route (104). The formation of antibodies as the result of an immune response can have a significant effect on the PK, efficacy, and safety of a therapeutic mAb $(104,105)$. A population $\mathrm{PK}$ analysis of infliximab in patients with ankylosing spondylitis found that patients positive for antibodies against infliximab had a $42 \%$ higher clearance than the remaining patients (36). However, when concentrations below the lower limit of quantification (LLOQ) were imputed as one-half of the LLOQ (originally these values were removed from the dataset), patients positive for antibodies against infliximab had a 
$77 \%$ higher clearance. The influence of antibody formation on the clearance of infliximab was found to have a much more pronounced effect in a population analysis conducted in patients with inflammatory bowel disease (22). In this study, patients with antibodies against infliximab had an approximately $170 \%$ higher clearance versus other patients. Another population PK analysis of infliximab in patients with ulcerative colitis, found that patients who developed antibodies against infliximab had a $47 \%$ higher clearance than those who did not (37). The formation of antibodies was also identified as an important covariate in a population PK analysis of ustekinumab, where psoriasis patients with a positive immune response had a $36 \%$ higher CL/F (47).

\section{Blood Chemistries}

Blood chemistry tests related to hepatic and renal function were often evaluated as potential covariates in the population PK analyses, but were rarely identified as covariates. In the population PK analyses of pertuzumab (43), bevacizumab (28), and ustekinumab (47) blood chemistries were found to influence the PK of the mAb (Table 1-5). The effect of blood chemistries on $\mathrm{CL}_{\mathrm{T}}$ and $\mathrm{V}_{1}$ in these studies was in general minimal and not likely to be clinically relevant. It should be noted that in many of the population PK analyses the effect of marked hepatic and renal impairment could not be sufficiently evaluated, because patients included in the studies typically had adequate hepatic and renal function. A population PK analysis of infliximab in patients with ulcerative colitis found albumin to be a negative predictor of $\mathrm{CL}_{\mathrm{T}}$ (37). Similar observations were made in population PK studies of pertuzumab and ustekinumab (43, 47). In addition to IgG, the FcRn also binds and protects albumin from intracellular catabolism, thereby playing an important role in the homeostasis of both $\operatorname{IgG}$ and albumin $(72,106)$. The authors of the infliximab analysis suggested that a higher albumin concentration could be an indicator of an increased number of FcRn and a related reduction in the rate of infliximab elimination (37).

\section{Disease and Comorbidities}

Only a small number of population PK studies investigated the effect of disease or comorbidities on the PK of the mAb. The potential effect of disease on the PK of infliximab was investigated in a population analysis of patients being treated for either Crohn's disease or ulcerative colitis (22). No difference in the PK of infliximab was observed between patients with Crohn's disease $(n=30)$ and ulcerative colitis $(n=3)$, but the small number of patients with ulcerative colitis may have precluded the ability to detect a possible difference. A number of comorbidities including diabetes, hypertension, and hyperlipidemia, were investigated as covariates in a population PK analysis of ustekinumab in psoriasis patients (47). This study found that patients with diabetes had a $28.7 \%$ and $13.2 \%$ higher $\mathrm{CL} / \mathrm{F}$ and $\mathrm{V} / \mathrm{F}$ for ustekinumab, respectively. The reason behind the effect of diabetes on the PK of ustekinumab is uncertain, but the authors suggested that altered lymphatic flow and particle transport, altered capillary permeability, increased interstitial fluid volume, and accelerated clearance of the 
antibody resulting from increased glycation could be potential mechanisms (47). Based on efficacy and safety data, the authors did not recommend that dose adjustments be made for diabetes. The potential effect of cancer type was investigated in a population $\mathrm{PK}$ analysis of panitumumab in cancer patients with various solid tumors (42). Patients with non-small cell lung cancer or other types of cancer had a smaller $\mathrm{CL}_{\mathrm{L}}(\sim 13-14 \%)$ compared to patients with colorectal or renal cancer (42). The reason for the difference in $\mathrm{CL}_{\mathrm{L}}$ between the different types of cancer is uncertain and was not addressed by the authors.

\section{Residual and Interoccasion Variability}

Residual variability in most of the population PK studies was modeled using either a proportional or a combined additive and proportional error model. In a few studies, an exponential error model was used to describe residual variability $(29,31,35)$. Estimates of residual variability from the $\mathrm{mAb}$ population $\mathrm{PK}$ studies are summarized in Table 1-6. Residual variability in the population PK analyses tended to be small to moderate. Based on the values in Table 1-6, the median (range) proportional residual error across all of the studies was $21.2 \% \mathrm{CV}(8.8-42)$. The proportional residual errors were relatively comparable across the different studies, while the additive residual errors were notably different. The additive component of a residual variance model often corresponds to the lower limit of quantification of an assay. Therefore, the large differences in the additive residual errors may be due in part to the different analytical assays used for different mAbs.

Residual variability can be dependent on a number of patient or study related factors, and these can be incorporated into the residual variance model as covariates (13). In most of the mAb population PK analyses, a simple residual error model with no covariates was used. However, in a population PK analysis of cetuximab, separate residual error models were used for the two clinical studies included in the analysis (29). This was done because of the unbalanced sampling design of the two studies (i.e., extensive versus sparse sampling), and their performance in different clinical settings.

In addition to between-subject and residual variability, another source of random variability that can be incorporated into the population PK model is interoccasion variability (IOV). The inclusion of IOV on model parameters was investigated for only a few mAbs. In a recent population PK analysis of matuzumab, IOV was included on $\mathrm{CL}_{\mathrm{L}}$ (40). Each infusion of matuzumab (up to the eighth infusion) was defined as one occasion. Interoccasion variability in $\mathrm{CL}_{\mathrm{L}}$ was $23 \% \mathrm{CV}$; however, this was very close to the estimated between-subject variability in $\mathrm{CL}_{\mathrm{L}}$ of $24 \% \mathrm{CV}$. The incorporation of interoccasion variability was also found to improve the model in a population $\mathrm{PK}$ analysis of sibrotuzumab (45). Although sibrotuzumab was administered as an IV infusion, IOV was found to be best included in the disposition on F1 or the "bioavailability" in the central compartment. Interoccasion variability in F1 was 13 $\% \mathrm{CV}$, and was lower than between-subject variability in the PK parameters which ranged from $20-52 \% \mathrm{CV}$. 


\section{Conclusion}

A review of the literature has shown that the population PK of different therapeutic $\mathrm{mAbs}$ are quite similar despite differences in their pharmacologic targets and being studied in different patient populations and disease states. A two-compartment model was used in the majority of the population analyses to describe the disposition of the $\mathrm{mAb}$. Population estimates for $\mathrm{V}_{1}$ and $\mathrm{V}_{2}$ were typically small with a median (range) of 3.1 L (2.4-5.5) and 2.8 L (1.3-6.8), respectively. The estimated between-subject variability in $\mathrm{V}_{1}$ was usually moderate with a median (range) of $26 \% \mathrm{CV}(12-84)$. Between-subject variability in other distribution related parameters such as $\mathrm{V}_{2}$ and $\mathrm{Q}$ were often not estimated. Although the most frequent PK models used in the population analyses were models with linear elimination, other models with nonlinear or parallel linear and nonlinear elimination pathways were also applied as many therapeutic mAbs are eliminated via saturable target-mediated mechanisms. Population estimates of $V_{\max }$ and $\mathrm{K}_{\mathrm{m}}$, the parameters characterizing Michaelis-Menten-type saturable elimination pathways, varied considerably among the different therapeutic mAbs. However, estimates for $\mathrm{CL}_{\mathrm{T}}$ in $\mathrm{mAbs}$ with linear elimination characteristics and $\mathrm{CL}_{\mathrm{L}}$ in $\mathrm{mAbs}$ with parallel linear and nonlinear elimination were quite similar among the different $\mathrm{mAbs}$ and typically ranged from around $0.2-0.5 \mathrm{~L} /$ day, which is relatively close to the estimated clearance of endogenous IgG of $0.21 \mathrm{~L} /$ day. The between-subject variability in $\mathrm{V}_{\max }$, $\mathrm{CL}_{\mathrm{T}}$, and $\mathrm{CL}_{\mathrm{L}}$ was moderate to large with estimates ranging from 15-65 \%CV. Measures of body size were the most commonly identified covariates found to influence the PK of therapeutic mAbs.

The similarities in the population PK of therapeutic mAbs are likely in part due to general structural features that are shared among most therapeutic mAbs as IgG molecules such as a functional human Fc region. One could hypothesize that targetbinding specific PK parameters such as $\mathrm{V}_{\max }$ and $\mathrm{K}_{\mathrm{m}}$ are likely to be influenced by the interaction between a given therapeutic $\mathrm{mAb}$ and its target and thus show high variability among different $\mathrm{mAbs}$, while the differences among parameters such as $\mathrm{CL}_{\mathrm{L}}, \mathrm{V}_{1}, \mathrm{~V}_{2}$, and $Q$ that are thought to be largely affected by the general structural features of $m A b s$ are minimal. In fact, the biggest differences observed in the population PK between the considered $\mathrm{mAbs}$ were for population estimates of $\mathrm{V}_{\max }$ and $\mathrm{K}_{\mathrm{m}}$. For the most part, no significant differences were observed in the population PK of murine, chimeric, humanized, and fully human therapeutic mAbs. The most notable difference observed between the different classes of $\mathrm{mAbs}$ was the larger population clearance estimates for the murine mAbs.

The PK characteristics of mAbs are very dissimilar compared to traditional smallmolecule drugs $(11,79)$, and tend to be more complex as mAbs often exhibit targetmediated drug disposition and nonlinear PK (4), which is oftentimes species-dependent and substantially complicates extrapolation of $\mathrm{mAb}$ PK characteristics from animal models to humans. When analyzing the population PK of a $\mathrm{mAb}$ it is therefore not only important to have a general understanding about the PK of mAbs, but to also understand 
the biology and pharmacology of the system. The complex PK characteristics of mAbs pose a significant challenge to a pharmacometrician performing a population PK analysis, as complex models are often evaluated to describe the mAb's PK, and the use of such models may be further complicated by limited concentration data. However, the similar population PK profiles of different therapeutic mAbs are an aspect of this class of biologics that can be used to the advantage of a more informed data analysis. The results of the studies summarized herein should be of value as they provide a general concept of what to expect with regard to applicable structural PK models, commonly identified covariates, and plausible estimates for typical population estimates, covariate effects, between-subject variability, and residual variability, which could be used for example to aid in choosing initial estimates or setting parameter boundaries. The compiled results from the population PK studies could also serve a more practical purpose and be used to construct informative priors for a Bayesian data analysis or for constructing parameter uncertainty distributions to simulate PK data for a prototypical therapeutic mAb. The ability to include prior information could prove to be particularly useful for $\mathrm{mAbs}$ as they often exhibit complex PK, and the use of complex PK models (e.g., parallel linear and nonlinear elimination pathways) may not be fully supported by available concentration data in datasets with sparse sampling and/or a limited number of dosage groups. 


\section{CHAPTER 2. RESEARCH HYPOTHESES}

Cetuximab is a therapeutic mAb directed against EGFR and is indicated in the treatment of squamous cell carcinoma of the head and neck (SCCHN). The PK of cetuximab has been well characterized in phase I studies indicating that it exhibits nonlinear elimination, but these studies have mainly been limited to noncompartmental analyses $(80,81,107)$, and the population PK of cetuximab has not been published in the literature except in abstract format for an analysis conducted for regulatory purposes $(108,109)$. I hypothesized that (I) nonlinear mixed effects modeling can be used to develop a population PK model that could adequately predict the systemic exposure of cetuximab in patients with SCCHN. Furthermore, I hypothesized that (II) covariates can be identified that are significant predictors for cetuximab systemic exposure. To address these hypotheses, I obtained cetuximab concentration-time data from two clinical trials in patients with SCCHN $(110,111)$, and a population PK analysis of the data with covariate analysis was carried out in Chapter 3.

The population PK analysis found that the PK of cetuximab was best described using a two-compartment model with nonlinear elimination. During the analysis, a number of issues and challenges were encountered which were likely the result of an attempt to describe this nonlinearity. The difficulty characterizing the nonlinear elimination of cetuximab was likely due to limitations of the clinical study designs, but may also have been due to the use of the FOCE estimation method in NONMEM throughout the analysis. It is known that FOCE, in addition to the first-order (FO) and Laplacian (LAP) estimation methods in NONMEM, can be inaccurate when dealing with highly nonlinear models such as the PK model with nonlinear elimination used to describe the PK of cetuximab (112). These estimation methods in NONMEM linearize the nonlinear mixed effects model (e.g., a population PK model) with regards to the between-subject random effects and then parameter estimates are obtained based on the linear approximation to the nonlinear model $(13,113)$. Therefore, I hypothesized that (III) other parameter estimation methods that do avoid linearization lead to more accurate parameter estimates and better characterization of the nonlinear PK encountered with monoclonal antibodies such as cetuximab.

One available method for parameter estimation without linearization is a full Bayesian analysis using Markov Chain Monte Carlo (MCMC) methods such as that implemented in the software package WinBUGS (114). One advantage of Bayesian MCMC methods over the FO, FOCE, and LAP methods in NONMEM is that the Bayesian MCMC methods do not rely on analytical approximations of the nonlinear mixed effects model, but instead use Monte Carlo integration techniques to obtain parameter estimates for the exact model $(115,116)$. Another advantage of Bayesian MCMC methods is the ability to include prior knowledge about the model parameters into the Bayesian model.

Nonlinear elimination is a common characteristic of the PK of therapeutic mAbs $(6,8)$, and accordingly, PK models with nonlinear elimination were used in almost half of 
the population PK analyses of therapeutic mAbs published in the scientific literature (Table 1-1). Therefore, the question of how well other population estimation methods perform in evaluating the nonlinear elimination of cetuximab is also relevant for other therapeutic mAbs. This notion is supported by the fact that similar difficulties encountered during the cetuximab analysis were also reported in population $\mathrm{PK}$ analyses of other therapeutic mAbs (Chapter 1).

To address my third hypothesis, I conducted a simulation study comparing the parameter estimation performance of the FO, FOCE with interaction (FOCE-I), and LAPI methods in NONMEM and a Bayesian MCMC method in WinBUGS when applied to population PK modeling of therapeutic mAbs with nonlinear PK. The Bayesian MCMC method was evaluated with uninformative and informative priors, which were obtained from population $\mathrm{PK}$ analyses of therapeutic mAbs in the literature. I evaluated the estimation methods under a dose-ranging design (informative study design) and four different single dose level designs at different dose levels (uninformative study designs). I made the following three subhypotheses: (IIIa) Under all study designs the FO method is less accurate and precise compared to the other estimation methods; (IIIb) When sufficient data is available to characterize the nonlinear $\mathrm{PK}$ of the therapeutic $\mathrm{mAb}$, as in the case of the informative design, the estimation performance of FOCE-I, LAP-I, and Bayesian MCMC (with both sets of priors) is adequate and comparable for all methods; (IIIc) Under the less-than-optimal (uninformative) study designs the estimation performance of the FOCE-I and LAP-I methods declines compared to the informative study design, while the formal inclusion of prior information with the Bayesian MCMC method provides a clear performance advantage over the other methods. 


\section{CHAPTER 3. POPULATION PHARMACOKINETICS OF CETUXIMAB IN PATIENTS WITH SQUAMOUS CELL CARCINOMA OF THE HEAD AND NECK*}

\section{Introduction}

Cetuximab is a recombinant, human/mouse chimeric IgG1 mAb that binds specifically to the extracellular domain of the human EGFR (also known as ErbB1/HER1) (117). By binding to EGFR, cetuximab prevents the binding of endogenous ligands such as epidermal growth factor and transforming growth factor- $\alpha$ (118). This blockade prevents EGFR signaling which is intricately involved in multiple processes involved in tumor growth and metastasis, such as cell proliferation, cell differentiation, cell survival, cell migration, tumor angiogenesis, and DNA repair $(119,120)$. Cetuximab can also induce internalization and subsequent degradation of EGFR, leading to downregulation of cell surface EGFR and a reduction in EGFR signaling $(121,122)$. Cetuximab is currently indicated in SCCHN and EGFR-expressing metastatic colorectal cancer (108). It is approved as a single agent in both cancers, and in combination therapy with radiation therapy for SCCHN and with irinotecan for colorectal cancer.

In early phase I studies, attempts to characterize the PK of cetuximab indicated that it exhibits nonlinear or Michaelis-Menten type elimination $(81,123)$. This nonlinearity was attributed to saturation of the EGFR-mediated endocytosis of cetuximab, a clearance pathway that entails internalization and degradation of the EGFR/cetuximab complex (80). While this nonlinear elimination pathway seems to be largely saturated at therapeutic concentrations, a second, nonspecific elimination pathway that is not saturable at therapeutic concentrations seems to be the major determinant for cetuximab elimination during therapy with approved cetuximab doses (109). The resulting elimination half-life estimates at therapeutic concentrations range between 66 and $97 \mathrm{hr}$ at the approved dosing regimen of $400 \mathrm{mg} / \mathrm{m}^{2}$ initial dose followed by weekly doses of $250 \mathrm{mg} / \mathrm{m}^{2}$, but increase from 33.3 to $119.4 \mathrm{hr}$ after single cetuximab doses of 20 to $500 \mathrm{mg} / \mathrm{m}^{2}$ (109).

Although cetuximab concentrations have been correlated with antitumor activity (124), most reports on cetuximab PK have been limited to noncompartmental analyses, and the population PK of cetuximab has not been published in the literature except in abstract format for an analysis conducted for regulatory purposes $(108,109)$.

Thus, this chapter describes a population PK analysis based on data from two clinical trials in patients with SCCHN. The objectives of this analysis were 1) to determine the typical population PK parameters for cetuximab and their between-subject variability, and 2) to identify covariates that are significant predictors for cetuximab

*This chapter adapted with permission. Dirks NL, Nolting A, Kovar A, Meibohm B. Population pharmacokinetics of cetuximab in patients with squamous cell carcinoma of the head and neck. J Clin Pharmacol 2008;48(3):267-78. 
systemic exposure and to assess their potential implications for clinical dosing.

\section{Methods}

\section{Study Design and Patient Population}

Cetuximab serum concentration-time data were obtained from two studies, EMR 62202-008 (study A) and EMR 62202-016 (study B), that are described elsewhere in detail $(110,111)$. Both studies were conducted in compliance with the Declaration of Helsinki and the Guidelines for Good Clinical Practice. Written informed consent was obtained from all subjects.

Study A was a randomized, multi-center phase I/II study $(n=53)$ to evaluate the safety and tolerability of cetuximab (Erbitux $®)$ in combination with platinum and 5fluorouracil (5-FU) as first-line treatment in patients with recurrent and/or metastatic $\mathrm{SCCHN}$, and to evaluate the PK of cetuximab under coadministration with platinum (110). Main inclusion criteria were stage III/IV recurrent or metastatic disease not suitable for local therapy, Karnofsky performance status $\geq 70$, and adequate hematologic, liver, and renal function. Cetuximab was administered as an initial dose of $400 \mathrm{mg} / \mathrm{m}^{2}$ infused over 2 hours, followed by weekly doses of $250 \mathrm{mg} / \mathrm{m}^{2}$ given over 1 hour. Patients were randomized to receive either cisplatin $100 \mathrm{mg} / \mathrm{m}^{2}$ on day 2 of the chemotherapy cycle or carboplatin AUC 5 (target area under the curve of $5 \mathrm{mg} / \mathrm{mL} *$ min) on day 1 of the cycle. 5-FU was administered as a continuous infusion on day 1 for the duration of 120 hours at the assigned dose levels of 600,800 , and $1000 \mathrm{mg} / \mathrm{m}^{2} /$ day. Chemotherapy (5-FU and platinum) cycles were repeated every 3 weeks. The primary treatment phase duration was 6 weeks ( 2 cycles), but patients were allowed to continue with treatment if they benefited from the initial treatment phase. Of the 53 patients enrolled into the study, 38 completed the primary treatment phase. Peak and trough cetuximab concentrations were obtained at weeks 2-4 of therapy. In addition to the peak and trough obtained on week 4 , a full concentration-time profile was obtained with sampling at 3, 6, 10, 24, 48, 72, 96 and 168 hours after the start of cetuximab administration.

Study B was a multi-center phase II study $(n=103)$ to evaluate the efficacy and toxicity of cetuximab (Erbitux $®)$ monotherapy in patients with platinum-refractory recurrent and/or metastatic SCCHN, and to investigate the PK of cetuximab (111). Main inclusion criteria were stage III/IV recurrent or metastatic disease, a Karnofsky performance status $\geq 60$, progressive disease documented by CT or MRI on platinumbased therapy, and adequate hematologic, liver, and renal function. Patients were to receive cetuximab as an initial infusion of $400 \mathrm{mg} / \mathrm{m}^{2}$ followed by weekly infusions of $250 \mathrm{mg} / \mathrm{m}^{2}$ for at least six weeks, if possible. If they responded to treatment or had stable disease, treatment was continued until disease progression, clinical deterioration, or unacceptable side effects were observed. On occurrence of disease progression or

clinical deterioration salvage treatment was offered with cetuximab $\left(250 \mathrm{mg} / \mathrm{m}^{2}\right.$ weekly $)$ 
plus the same platinum regimen the patient was on prior to receiving cetuximab monotherapy. Of the 103 patients treated with cetuximab monotherapy, 53 subsequently received combination therapy. Cetuximab peaks and troughs were obtained at weeks 1 , 4 , and 6 during monotherapy. If the patient entered the combination therapy phase, cetuximab peaks and troughs were obtained again at weeks 1, 4, and 6 after switching to combination therapy. A sample was also taken at the end-of-study visit, which was up to six weeks after the last dose of cetuximab was administered. The total study period ranged from 0.1 to 56 weeks with a median of 14 weeks.

\section{Assay Methodology}

Cetuximab serum concentrations were determined using a validated sandwich enzyme-linked immunosorbent assay (ELISA). In brief, serum samples were diluted by a factor of 500 and added to microtiter plates coated with the extracellular domain of EGFR, which served as the capture antigen. After incubation, plates were washed with buffer and further incubated with rabbit anti-human IgG conjugated with horseradish peroxidase, which binds to the cetuximab immobilized in the microtiter plates. Unbound conjugate was removed, and tetramethylbenzidine, a substrate for horseradish peroxidase, was added. Tetramethylbenzidine was oxidized to a colored product, the absorbance of which, was measured by a plate reader at $450 \mathrm{~nm}$. This absorbance was directly related to the cetuximab concentration in the serum sample. The lower and upper limits of quantification were $0.5 \mathrm{ng} / \mathrm{mL}$ and $15 \mathrm{ng} / \mathrm{ml}$ for the diluted cetuximab samples, corresponding to 0.25 and $7.5 \mu \mathrm{g} / \mathrm{mL}$ for undiluted samples. Serum samples with higher concentrations of cetuximab were further diluted to fit into the linear calibration range. Accuracy of the assay was between 98.5 to $104 \%$, the precision ranged from 2.2 to $6.3 \%$.

\section{Population Pharmacokinetic Analysis}

The population PK analysis was performed by nonlinear mixed effects modeling using a NMQual (Metrum Institute, Augusta, ME) installation of NONMEM (version V, level 1.1; Icon Development Solutions, Ellicott City, MD) under Compaq Visual Fortran (version 6.5; Compaq Corporation, Houston, TX). Xpose (125), Census (126), and SPlus (version 7.0; Insightful Corporation, Seattle, WA) were used for data management and visualization. The FOCE method within NONMEM was used throughout the model building process (20). Across the two studies, the majority of the concentration measurements were within the first 8 weeks of cetuximab therapy. Therefore, the database was split with concentration data obtained during the first 8 weeks used for model building ('the model building dataset'), and the remaining data for model qualification ('the model evaluation dataset'). Sampling times in the model evaluation data set ranged from week 10 to 43 . The structural model was evaluated using only the densely sampled data from study A. The covariate model was subsequently built using data from both studies. All concentration data was log transformed prior to the analysis, and the residual variance model was modified accordingly (transform-both-sides 
approach). Log transformation of the data stabilized the convergence of the structural models and resulted in shorter run times.

Six structural models including one- or two-compartmental models with linear, nonlinear, or parallel linear and nonlinear elimination pathways were evaluated based on minimization of the Akaike Information Criterion (AIC) value, precision and plausibility of parameter estimates, and a number of goodness-of-fit plots. The AIC was defined in terms of the objective function value (OFV) and number of parameters ( $p)$ in the model: $\mathrm{AIC}=\mathrm{OFV}+2 \mathrm{p}$. Prior information about the PK of cetuximab from phase I and phase II trials, such as the presence of nonlinear elimination, was also taken into account while building the structural model.

Between-subject variability in cetuximab PK parameters was assumed to follow a lognormal distribution, and was modeled according to an exponential error model. During the model building procedure, between-subject variability for parameters was assumed to be uncorrelated, and only the diagonal elements of the variance-covariance matrix were estimated. Once the final model was determined, the addition of covariance terms was evaluated. Due to the unbalanced sampling design of the two studies and their performance in different clinical settings, separate residual error models were used per study. Residual variability was modeled using a log transformed exponential error model.

Twenty-one potential covariates were evaluated. The covariates were comprised of 1) demographic and anthropometric measurements: gender, age, ethnicity, WGT, IBW (127), BSA, and clinical study; 2) blood chemistry and hematology measurements: blood urea nitrogen, serum creatinine, creatinine clearance, total bilirubin, albumin, aspartate and alanine aminotransferases, and WBC count; 3 ) concomitant therapy and disease related factors: EGFR expression, presence of human anti-chimeric antibodies, concomitant platinum and/or 5-FU therapy, Karnofsky function score, and duration of cetuximab therapy. Covariates were updated over time in the observation records for each patient when the respective data were available. Before building the covariate model, all potential covariates were plotted against each other to identify any high intercorrelation, as to avoid simultaneous inclusion of correlated predictors (128).

The covariate model was built using a stepwise forward addition/backward deletion modeling approach (129). Inclusion of a covariate-parameter relationship was based on the likelihood ratio test. The criteria for including a covariate in the model during the forward addition steps was a decrease in the OFV of $3.84(\mathrm{p}<0.05)$. During the backward deletion steps, an OFV change of $10.83(\mathrm{p}<0.001)$ was required for a covariate to remain in the model. A more stringent criterion was used during the backward deletion steps because of the multiple comparisons made during the forward portion of stepwise covariate modeling. Covariate modeling was also guided by goodness-of-fit plots and changes in between-subject variability.

A stratified approach to the univariate analysis was taken during the forward addition step of the covariate modeling, in that size covariates (IBW, WGT, BSA) were 
first evaluated and included in the model. Once the effect of size on cetuximab PK had been accounted for, the remaining covariates were evaluated in the model. The stratified approach was taken as correlation often exists between size covariates and non-size covariates (e.g., gender and weight) (97). Such correlations can mask covariate relationships, and lead to difficulties interpreting the effects of two correlated covariates. Stepwise backward deletion was conducted once the full model was established.

Relationships between continuous covariates and PK parameters were modeled using linear proportional (Eq. 3-1) and power (nonlinear) models (Eq. 3-2) with the covariate $(\mathrm{COV})$ normalized to the population median for the dataset. Categorical covariates were modeled as shown in Eq. 3-3:

$$
\begin{gathered}
P=\theta_{1} \times\left(1+\theta_{2} \times[C O V-\text { Median }]\right) \\
P=\theta_{1} \times\left(\frac{C O V}{\text { Median }}\right)^{\theta_{2}} \\
P=\theta_{1} \times\left(1+\theta_{2} \times C O V\right)
\end{gathered}
$$

where the $\theta$ 's are the parameters to be estimated, and $\theta_{1}$ represents the typical value of a PK parameter $(\mathrm{P})$ in an individual with the median value for the covariate.

\section{Model Qualification}

The ability of the final population PK model to adequately describe the observed data in the model building dataset was evaluated using a predictive check. Cetuximab peak and trough concentrations at week 4 of therapy served as the metrics of interest. The final population PK model and parameter point estimates were used to perform Monte Carlo (parametric) simulations of the metrics of interest. The model evaluation dataset with concentration data beyond week 8 was used to evaluate the ability of the final population PK model to predict cetuximab concentrations in an independent dataset. Nonparametric bootstrap analysis was used to evaluate the precision and stability of the final model parameter estimates. Patients were randomly sampled with replacement from the model building dataset used for building the population PK model to create bootstrap datasets with the same sample size as the original. A stratified sampling approach was used in that the ratio of patients from the two studies was kept constant. 


\section{Results}

\section{Data}

A total of 143 patients provided evaluable PK data for the population analysis, 47 from study A and 96 from study B. Table 3-1 summarizes the demographics for these patients. The majority of patients $(138 / 143)$ had EGFR expressing tumors determined by immunohistochemical evaluation. The presence of human anti-chimeric antibodies was not detected in any of the patients participating in the two studies.

The initial cetuximab dataset consisted of 1,142 concentration measurements. There were 86 baseline observations prior to the first cetuximab administration, which were defined as zero and removed from the dataset before log transformation. After splitting the database into a model building and a model evaluation dataset, 912 concentrations remained for building the population PK model, 530 from study A and 382 from study B. Due to the unbalanced sampling schedule between the two studies, study A patients contributed a median of 13 data points per individual, while study B patients contributed a median of 4 data points per individual.

\section{Structural Model}

Cetuximab serum concentrations were best described by a two-compartment model with nonlinear elimination, based on AIC values and goodness-of-fit plots. Compared to a one- or two-compartment model with linear elimination, the corresponding model with nonlinear elimination resulted in further reduction of the AIC value. The goodness-of-fit plots indicated that the nonlinear model provided a better fit to lower concentrations $(<75 \mu \mathrm{g} / \mathrm{mL})$ than the linear model. In addition, the results from prior PK studies supported the use of a nonlinear elimination model $(80,81,123)$.

Addition of a second, linear elimination pathway to the nonlinear model resulted in no change in the OFV. The goodness-of-fit plots between the nonlinear model and parallel elimination model were very similar. This lack of improvement in fit to the data with the parallel elimination model led to selection of the more parsimonious nonlinear model as the structural base model. Regardless of the elimination pathways, a two-compartment model always provided a better fit to the data than a one-compartment model based on AIC values and goodness-of-fit plots. The two-compartment nonlinear elimination model was parameterized in terms of $\mathrm{V}_{\max }, \mathrm{K}_{\mathrm{m}}, \mathrm{V}_{1}, \mathrm{~V}_{2}$, and $\mathrm{Q}$.

The initial structural model was unable to account for between-subject variability in both $\mathrm{V}_{\max }$ and $\mathrm{K}_{\mathrm{m}}$, even after addition of a covariance term for both parameters. Based on the hypothesis that physiologically there should be more variability among individuals with regard to $V_{\max }$ than $\mathrm{K}_{\mathrm{m}}$, the $\mathrm{K}_{\mathrm{m}}$ parameter was treated as a fixed value across all subjects, which resulted in an OFV increase of only 0.3 points and improvement of the precision for the estimate of between-subject variability in $\mathrm{V}_{\max }$. 
Table 3-1. Patient characteristics.

\begin{tabular}{llll}
\hline Characteristics & N & Median & Range \\
\hline Gender (male/female) & $120 / 23$ & & \\
Ethnicity (caucasian/unknown) & $131 / 12$ & & \\
Study (A/B) & $47 / 96$ & & \\
Concomitant 5FU' (yes/no) & $47 / 96$ & & \\
Concomitant platinum ${ }^{\mathrm{a}}$ (yes/no) & $89 / 54$ & & $23-77$ \\
Age & & 56 & $1-54$ \\
Duration of cetuximab therapy (week) & 6 & $34-113$ \\
Weight (kg) & 60 & $1.2-2.2$ \\
BSA (m²) & 1.7 & $43.3-81.3$ \\
IBW (kg) & 64.2 & $1.7-41.8$ \\
White blood cell count (10 $9 / \mathrm{L})$ & 6.8 & $4-106$ \\
Blood urea nitrogen (mg/dl) & & 23 & $0.4-1.9$ \\
Serum creatinine (mg/dl) & & 0.9 & $22-167$ \\
Creatinine clearance (ml/min) & 74 & $0.2-1.4$ \\
Total bilirubin (mg/dl) & & 0.5 & $1.3-4.8$ \\
Albumin (g/dl) & & 3.9 & $5-150$ \\
Aspartate aminotransferase (U/L) & & 18 & $1-64$ \\
Alanine aminotransferase (U/L) & & 14 & $60-100$ \\
Karnofsky function score & 80 & \\
\hline
\end{tabular}

a. Patient received concomitant 5-fluorouracil (5-FU) or platinum chemotherapy at some point during the trial. 
Once the base model was determined, data from study A and B were pooled together for the covariate modeling. Separate residual error models were used for each study, which added stability to the minimization procedure and resulted in a change in objective function value $(\triangle \mathrm{OFV})$ of -32.8 , as well as a decrease in between-subject variability estimates for all parameters.

\section{Covariate Model}

The effect of covariates on cetuximab PK was first investigated for size measures with the purpose of identifying which, if any, are the most influential. The $\triangle \mathrm{OFV}$ indicated that linearly related IBW $(\triangle \mathrm{OFV}-29)$ was the most important size covariate for $\mathrm{V}_{\max }$ versus BSA ( $\left.\triangle \mathrm{OFV}-14\right)$ and WGT $(\Delta \mathrm{OFV}-9)$. Inclusion of IBW reduced the between-subject variability in $\mathrm{V}_{\max }$ from $23.6 \%$ to $17.9 \%(\% \mathrm{CV})$. Based on $\Delta \mathrm{OFV}$, linearly related WGT was found to be the most important size covariate on both $\mathrm{V}_{1}$ and $\mathrm{V}_{2}$. Inclusion of WGT on $\mathrm{V}_{1}$ resulted in a $\triangle \mathrm{OFV}$ of -22.7, and a decrease in betweensubject variability for $\mathrm{V}_{1}$ from $21 \%$ to $18.3 \%$. Adding WGT as a covariate on $\mathrm{V}_{2}$ resulted in a $\triangle \mathrm{OFV}$ of -9.7 , and a decrease in between-subject variability for $\mathrm{V}_{2}$ from $54.1 \%$ to $46.6 \%$. No effect of size covariates was identified for $\mathrm{Q}$ or $\mathrm{K}_{\mathrm{m}}$.

The remaining covariates were screened using general additive modeling (130) and plots of individual empirical Bayes estimates of the parameters versus each of the covariates. The general additive modeling only identified $\mathrm{WBC}$ as a covariate on $\mathrm{V}_{\max }$, while graphical analysis identified the same relationship as well as a potential effect of gender on $\mathrm{V}_{\max }, \mathrm{V}_{1}$, and $\mathrm{V}_{2}$. Modeling $\mathrm{V}_{\max }$ as a linear function of WBC resulted in a $\triangle \mathrm{OFV}$ of -37.0 and decreased the between-subject variability for $\mathrm{V}_{\max }$ further to $15.3 \%$. No effect of gender was identified for $\mathrm{V}_{\max }(\Delta \mathrm{OFV}-0.3)$ or $\mathrm{V}_{2}(\Delta \mathrm{OFV}-2.4)$. Adding gender as a covariate for $\mathrm{V}_{1}$ resulted in a $\triangle \mathrm{OFV}$ of -6.4 , but reduced the between-subject variability only from $18.7 \%$ to $17.8 \%$. The estimated difference in $\mathrm{V}_{1}$ between males and females was small (13.5\% smaller $\mathrm{V}_{1}$ for females). Subsequently, the effect of gender on $\mathrm{V}_{1}$ did not pass the criterion for backward deletion and was removed from the model. The effect of WGT on $\mathrm{V}_{2}$ was also removed during the backward deletion step, but all other covariates were retained in the model. The addition of covariance terms between $\mathrm{V}_{\max }$ and the volume parameters was investigated with the final model, but did not result in any improvement.

The final population PK model and parameter estimates are presented in Table 3-2. The NONMEM control stream and run output for the final population PK model are included in Appendix A. Goodness of fit plots for the final model are shown in Figure 3-1, and model-predicted and measured cetuximab concentration-time profiles for representative patients are provided in Figure 3-2. The estimates for $V_{\max }$ and $K_{m}$ for a typical patient with median covariate values, as defined in the model, were $4.38 \mathrm{mg} / \mathrm{hr}$ and $74 \mu \mathrm{g} / \mathrm{mL}$, respectively. Between-subject variability in the parameters was relatively small for $\mathrm{V}_{\max }(15.4 \%)$ and $\mathrm{V}_{1}(18.6 \%)$, but was larger for $\mathrm{V}_{2}(56.4 \%)$ and $\mathrm{Q}(97.2 \%)$. IBW and WBC accounted for almost $35 \%$ of the total variability in $\mathrm{V}_{\max }$, while WGT 
Table 3-2. Final population pharmacokinetic model and its parameter estimates with bootstrap-derived $90 \%$ confidence intervals.

\begin{tabular}{|c|c|c|c|c|c|c|}
\hline Parameter & Model & & $\begin{array}{l}\text { Parameter } \\
\text { estimate }\end{array}$ & $\begin{array}{l}\text { Parameter } \\
90 \% \mathrm{CI}\end{array}$ & $\begin{array}{l}\text { BSV } \\
(\% \mathrm{CV})^{\mathrm{a}}\end{array}$ & $\begin{array}{l}\text { BSV } \\
90 \% \mathrm{CI}\end{array}$ \\
\hline \multirow[t]{3}{*}{$\mathrm{V}_{\max }[\mathrm{mg} / \mathrm{hr}]$} & $\begin{array}{l}\theta_{1} \times\left(1+\theta_{2} \times(\mathrm{IBW}-64)\right. \\
\left.+\theta_{3} \times(\mathrm{WBC}-6.8)\right)\end{array}$ & $\theta_{1}$ & 4.38 & $3.40,6.64$ & 15.4 & $12.0,19.1$ \\
\hline & & $\theta_{2}$ & 0.0108 & $0.0077,0.0139$ & & \\
\hline & & $\theta_{3}$ & 0.0216 & $0.0169,0.0296$ & & \\
\hline $\mathrm{K}_{\mathrm{m}}[\mu \mathrm{g} / \mathrm{mL}]$ & $\theta_{4}$ & $\theta_{4}$ & 74 & $38.2,163.3$ & $---{ }^{b}$ & \\
\hline \multirow[t]{2}{*}{$\mathrm{V}_{1}[\mathrm{~L}]$} & $\theta_{5} \times\left(1+\theta_{6} \times(\right.$ WGT-60) $)$ & $\theta_{5}$ & 2.83 & $2.69,2.96$ & 18.6 & $12.5,22.2$ \\
\hline & & $\theta_{6}$ & 0.0083 & $0.0057,0.0115$ & & \\
\hline $\mathrm{V}_{2}[\mathrm{~L}]$ & $\theta_{7}$ & $\theta_{7}$ & 2.43 & $1.95,2.85$ & 56.4 & $18.0,72.8$ \\
\hline $\mathrm{Q}[\mathrm{L} / \mathrm{hr}]$ & $\theta_{8}$ & $\theta_{8}$ & 0.103 & $0.062,0.191$ & 97.2 & $40.2,133$ \\
\hline \multirow{2}{*}{\multicolumn{2}{|c|}{ Residual variability $(\% \mathrm{CV})^{\mathrm{a}}[90 \% \mathrm{CI}]$ : }} & \multicolumn{5}{|c|}{ Study A: $14.6[12.3,16.8]$} \\
\hline & & \multicolumn{5}{|c|}{ Study B: $21.2[18.8,23.7]$} \\
\hline
\end{tabular}

$\mathbf{C I}=$ confidence interval; $\mathbf{B S V}=$ between-subject variability; $\mathbf{I B W}=$ ideal body weight $(\mathrm{kg}) ; \mathbf{W B C}=$ white blood cell count $\left(10^{9} / \mathrm{L}\right)$; WGT $=$ body weight $(\mathrm{kg})$.

a. Approximate $\% \mathrm{CV}$ calculated as $\sqrt{\omega^{2}} \times 100$ where $\omega^{2}$ is the between-subject variance estimate.

b. BSV not estimated for $\mathrm{K}_{\mathrm{m}}$. 
Figure 3-1. Goodness-of-fit plots for the final model. Plot of population and individual predicted versus observed cetuximab concentrations and plot of conditional weighted residuals versus population predicted concentrations and time (days). 

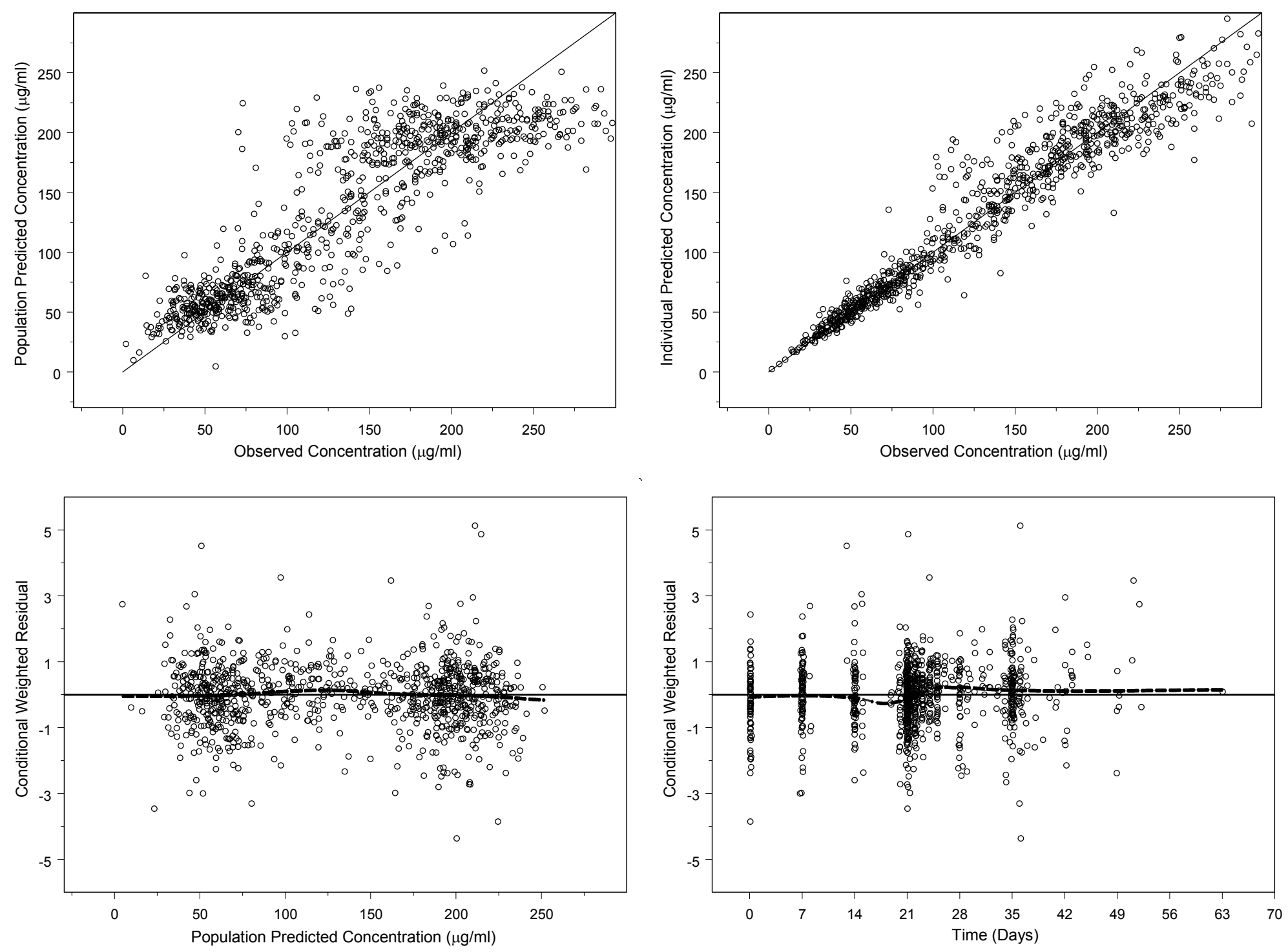


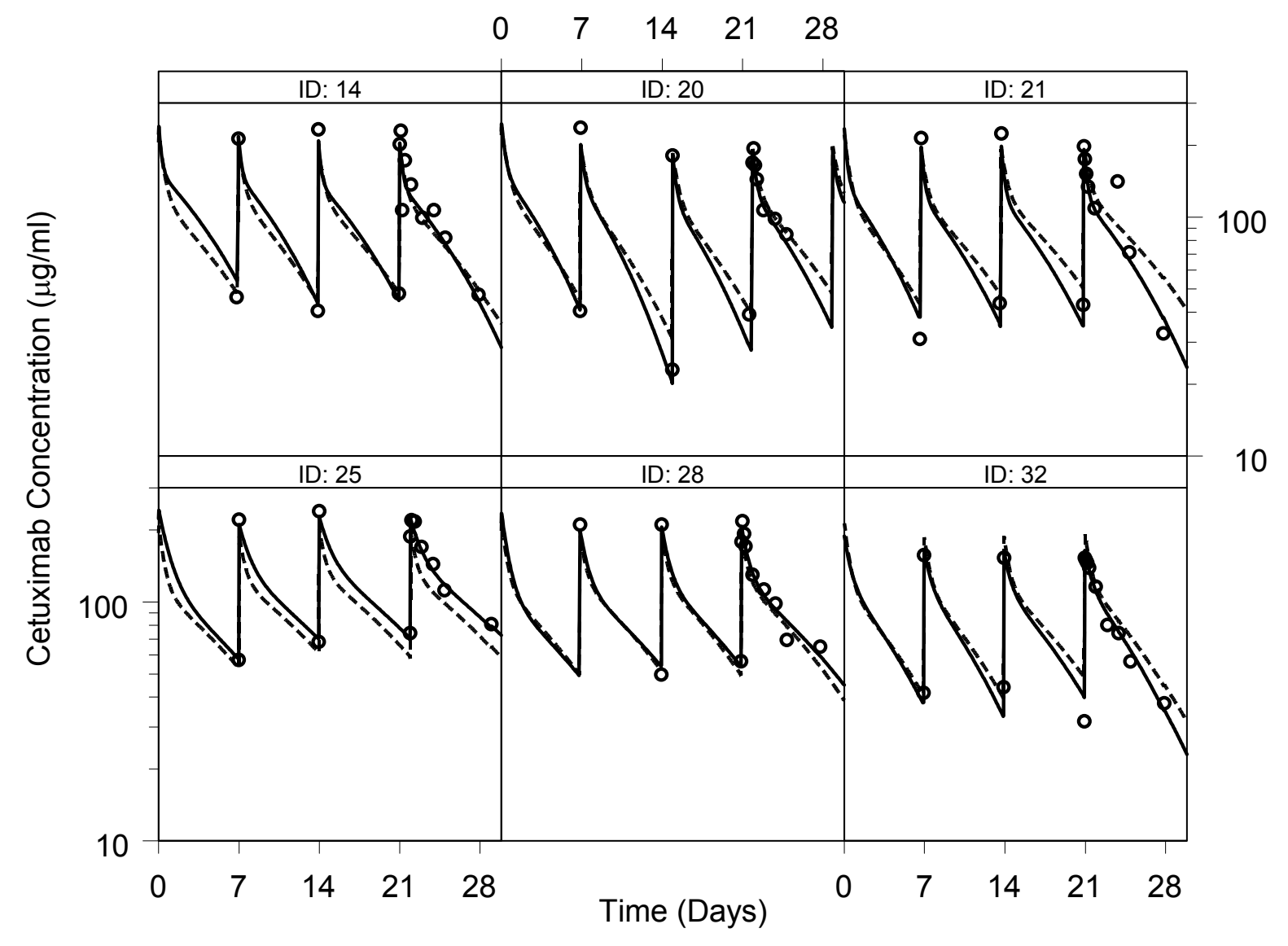

Figure 3-2. Cetuximab serum concentration-time profile in six representative patients (patient ID indicated) over a period of 28 days after initiation of therapy with a dose of $400 \mathrm{mg} / \mathrm{m}^{2}$, followed by weekly doses of $250 \mathrm{mg} / \mathrm{m}^{2}$. Measured serum concentrations are indicated by open circles, model-based population and individual predicted concentration-time profiles by dashed and solid lines, respectively. 
accounted for approximately $10 \%$ of the variability in $\mathrm{V}_{1}$. The estimate of the proportional residual variance for study A (14.6\%) was less than that for study B $(21.2 \%)$, which may be attributed to the highly unbalanced sampling designs of the two studies.

\section{Model Qualification}

The precision of model parameter estimates was assessed by 500 nonparametric bootstrap replicates. As shown in Table 3-2, the 90\% confidence intervals were relatively tight and indicated good precision of most parameter estimates. The median population estimates obtained from the nonparametric bootstrap were generally within $5 \%$ of the estimates from the final model (data not shown), indicating that the final population model was stable. Furthermore, the $95 \%$ confidence intervals for the covariate effects did not overlap with zero, indicating the statistical significance of the covariates included in the final model.

The model was further evaluated using a predictive check for the model building dataset and simulation of concentrations in the model evaluation data set. For both model qualification methods, 500 Monte-Carlo simulated datasets were created and compared to the observed peak and trough concentrations at week 4 for the model building dataset (Figures 3-3 and 3-4) and beyond week 8 for the model evaluation dataset (Figure 3-5), respectively. The model adequately described general tendencies of peak and trough concentrations and the between-subject variability of peaks at week 4 in the model building data set (Figures 3-3 and 3-4) and beyond week 8 of therapy in the model evaluation data set (Figure 3-5). Figure 3-3, however, indicates that the model overpredicted the between-subject variability in trough concentrations at week 4 at the lower concentrations, suggesting that one or more of the variance components of the model were over-estimated. This is reflected in Figures 3-4 and 3-5 where less than 10\% of the trough observations fell outside the simulated $90 \%$ prediction interval.

For the model evaluation dataset, the median prediction error and median absolute prediction error were calculated using the individually predicted and observed concentrations beyond week 8 . The median prediction error was $-0.1 \%$ and $0.3 \%$ and the median absolute prediction error was $2.9 \%$ and $6.8 \%$ for peak and trough concentrations, respectively, indicating that the predictions were generally unbiased and relatively precise.

\section{Discussion}

Therapeutic mAbs often exhibit nonlinear PK presumably attributable to targetmediated elimination pathways via interaction with their antigen (6). The binding of cetuximab to the membrane receptor EGFR presents a specific, saturable elimination mechanism by which the cetuximab/EGFR complex is internalized and either degraded or recycled with recurrence of the receptor at the cell surface (109). This receptor- 

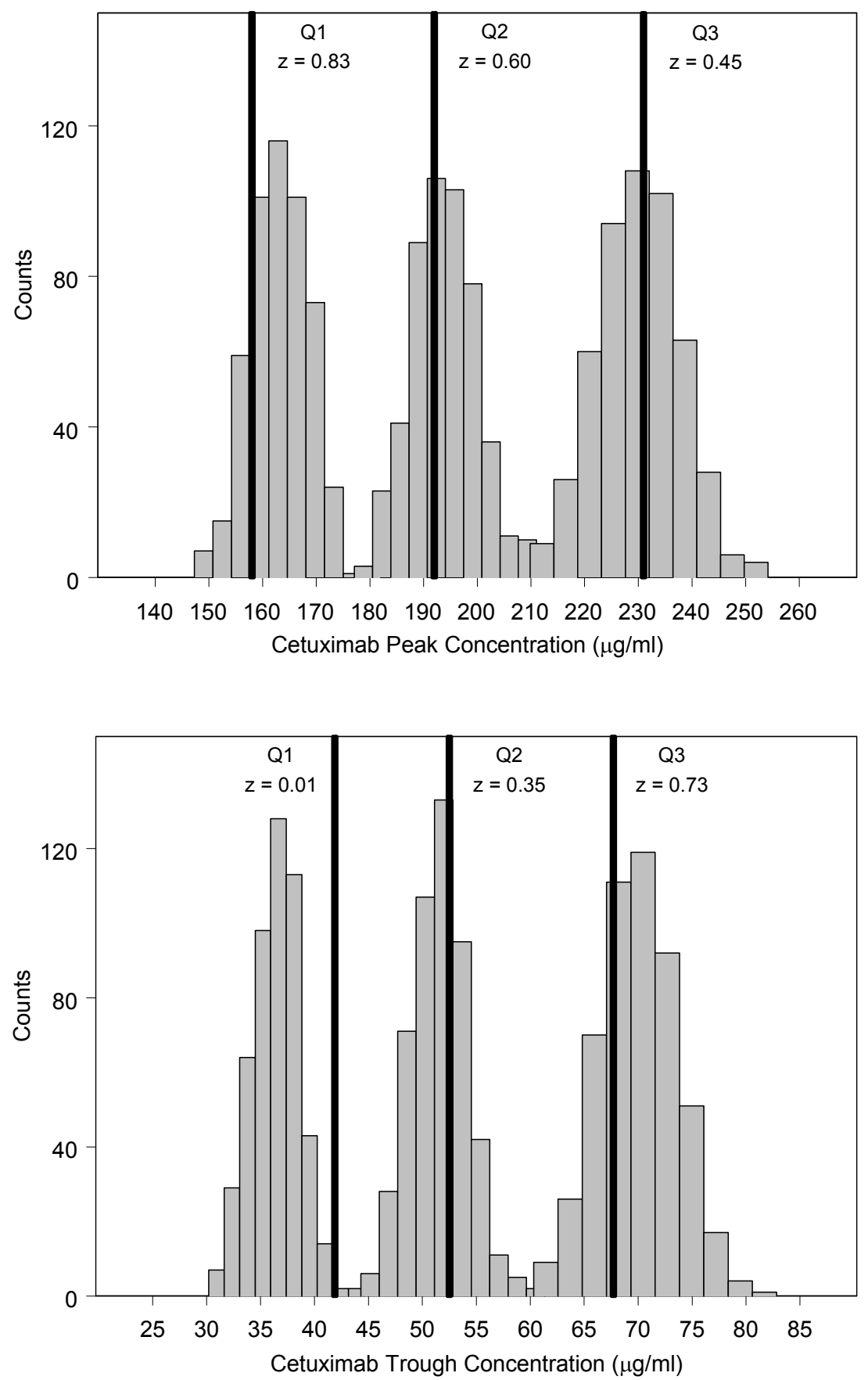

Figure 3-3. Results of the predictive check for peak and trough concentrations at week 4 of therapy. The histogram plot shows the distribution of peak (top panel) and trough (bottom panel) quartiles (Q1: $25^{\text {th }}$ percentile; Q2: median; Q3: $75^{\text {th }}$ percentile) for 500 simulated datasets, and the vertical lines indicate the quartiles for the observed data. The $\mathrm{z}$-value represents the fraction of the quartiles of the simulated data that are larger than the corresponding observed quartile. 


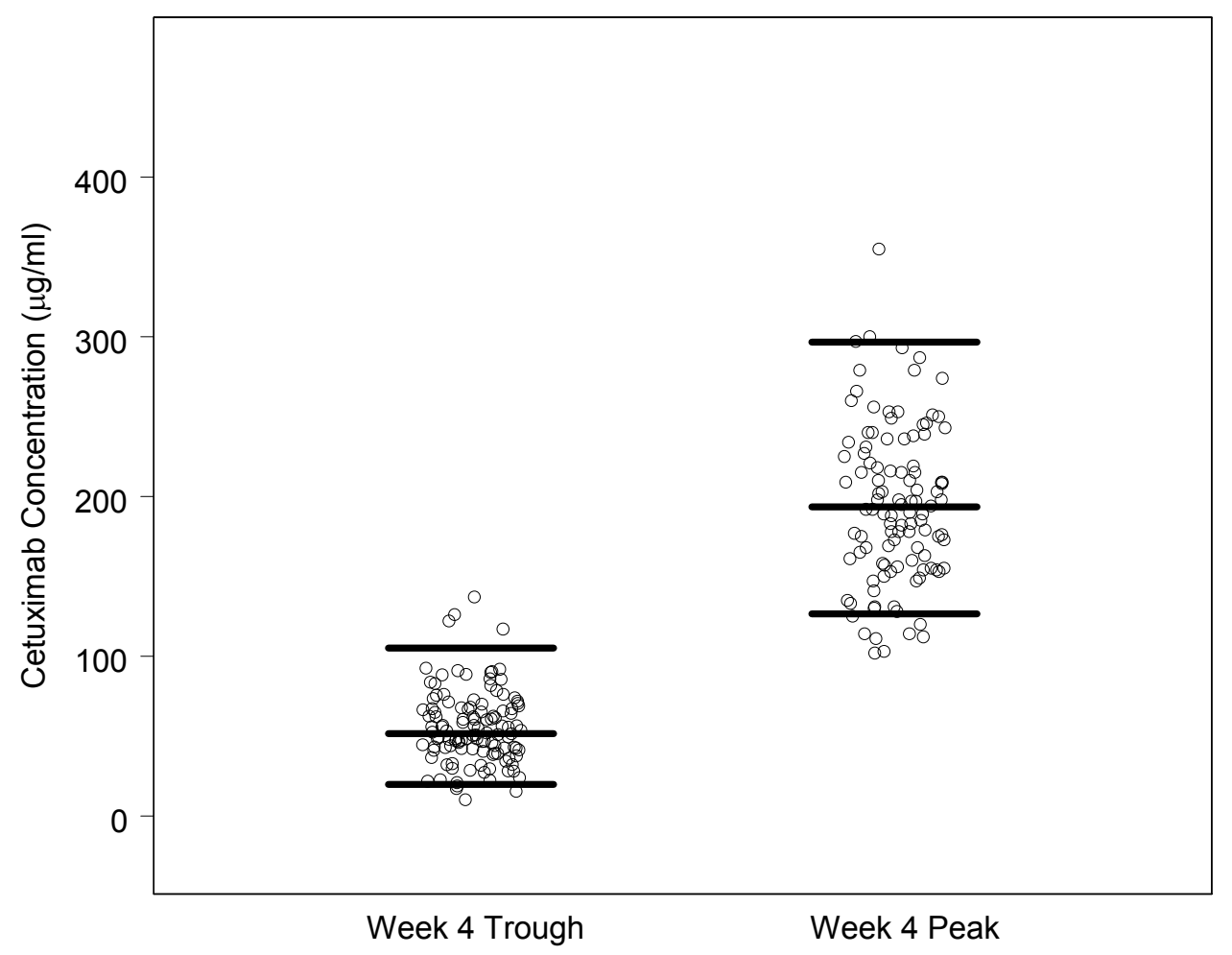

Figure 3-4. Scatterplot of the results from the predictive check of peak and trough concentrations at week 4 of therapy in the model building dataset. The solid lines indicate the peak and trough $5^{\text {th }}, 50^{\text {th }}$, and $95^{\text {th }}$ percentiles determined from all 500 simulated datasets. Observed data is plotted as individual points. 


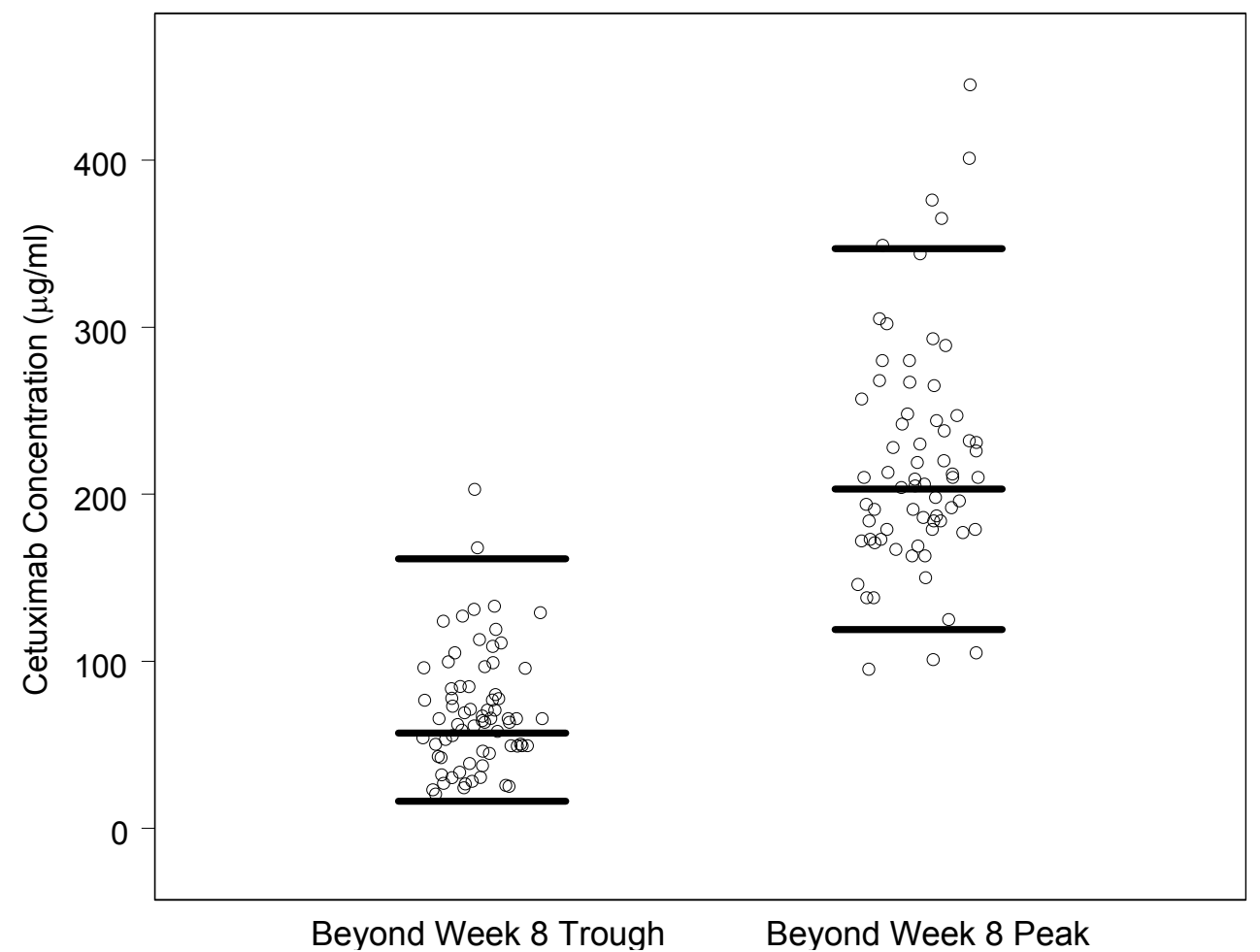

Figure 3-5. Results of the simulation of concentrations in the model evaluation dataset beyond week 8 of therapy. The solid lines indicate the peak and trough $5^{\text {th }}, 50^{\text {th }}$, and $95^{\text {th }}$ percentiles determined from all 500 simulated datasets. Observed data is plotted as individual points. 
mediated endocytosis likely contributes a significant portion to the total clearance of cetuximab, given that EGFR is widely expressed in a variety of tissues. Panitumumab, another $\mathrm{mAb}$ targeted towards EGFR, also exhibits nonlinear PK attributed to saturation of EGFR-mediated clearance (131). The effect of target-mediated clearance on therapeutic mAbs has also been observed for other membrane targets $(8,30)$.

The saturable, nonlinear PK of cetuximab has been well characterized in phase I studies $(80,81,123)$, where cetuximab clearance was shown to decrease with increasing doses in the range of 20 to $200 \mathrm{mg} / \mathrm{m}^{2}$. Likewise, in the current population analysis, I found that a two-compartment model with nonlinear elimination provided the best fit to the data. Phase I studies also showed that at doses of $200 \mathrm{mg} / \mathrm{m}^{2}$ and greater (up to 500 $\left.\mathrm{mg} / \mathrm{m}^{2}\right)$, the estimated clearance for cetuximab levels off $(80,81,109)$. The biphasic nature of cetuximab clearance versus dose suggests the presence of a nonsaturable firstorder process in parallel to the EGFR-mediated elimination described above (109). This nonspecific linear elimination process is common for all antibodies, and is likely due to slow proteolytic degradation from interaction between the Fc region of the antibody and Fc-receptors on hepatic cells or cells of the reticuloendothelial system $(8,109)$. An attempt was made to model this parallel elimination process by building a model with both linear and nonlinear elimination pathways as it has previously been used in the PK analysis of other therapeutic mAbs, including sibrotuzumab and panitumumab $(45,131)$. The parallel elimination model did not offer a significant improvement in describing the data over the simpler nonlinear model likely because the data did not support the more complex model. Certainly, a limitation to this analysis was that dose escalation data was not available as all patients included in the analysis were treated at the same clinically approved dose level. Such data might have supported the more complex parallel elimination model, and even helped further define the population estimates and betweensubject variability for $\mathrm{V}_{\max }$ and $\mathrm{K}_{\mathrm{m}}$.

Based on the final population PK model, cetuximab distributes into a central compartment volume $\left(\mathrm{V}_{1}\right)$ of $2.83 \mathrm{~L}$ for a patient weighing $60 \mathrm{~kg}$, which is approximately equal to the plasma volume. The volume of distribution at steady-state $\left(V_{s s}=V_{1}+V_{2}\right)$ of $5.26 \mathrm{~L}$ for a $60 \mathrm{~kg}$ patient suggests an apparently limited distribution outside the vascular space, which is in line with the results from cetuximab phase I studies $(80,81,109)$, and consistent with the behavior of endogenous $\operatorname{IgG}$ and other therapeutic mAbs $(6,132)$. Nevertheless, cetuximab is able to distribute to its target as evidenced by immunohistochemistry studies and EGFR tyrosine kinase assays, which showed almost complete EGFR saturation in tumor tissue samples obtained from patients with SCCHN receiving the approved doses of cetuximab (133).

The results of the predictive check and simulations revealed that the population PK model adequately described the central tendencies of peak and trough concentrations at week 4 in the model building data set and beyond week 8 in the model evaluation dataset. The model was able to adequately account for the between-subject variability in peak concentrations, but the variability in trough concentrations was overpredicted towards lower concentrations as indicated by the disparity between the $25^{\text {th }}$ percentiles (Q1) for observed and simulated trough concentration data in Figure 3-3. Given that $\mathrm{V}_{\max }$ 
significantly influences trough concentrations, overestimation of the between-subject variability in $\mathrm{V}_{\max }$ may be the cause for this deviation.

Binding of cetuximab to EGFR induces internalization of the receptor, which is believed to lead to EGFR down-regulation (109). Given the role EGFR plays in the elimination of cetuximab, one might hypothesize that with down-regulation of EGFR, there may also be a decrease in cetuximab clearance over time. Based on the Monte Carlo simulations for the model evaluation dataset, however, the population PK model was able to adequately describe cetuximab peak and trough concentrations beyond 8 weeks of therapy. This finding suggests that the elimination PK of cetuximab remains relatively stable over time during prolonged therapy. It is further supported by findings from a phase I study, where no statistically significant change in clearance values was found between the first and fourth weekly dose of cetuximab (81).

An interesting and unexpected covariate relationship identified in my analysis is the influence of WBC count on $\mathrm{V}_{\max }$. There is evidence in the literature, however, that lymphocytes, monocytes, macrophages, and neutrophils do express EGFR (134-136). This suggests that these cells may be involved in the receptor-mediated clearance of cetuximab. The final population PK model predicted a 2.2\% change in $V_{\max }$ per unit $\left(10^{9} / \mathrm{L}\right)$ change in WBC from the median WBC $\left(6.810^{9} / \mathrm{L}\right)$. Over the normal range of WBC $\left(\sim 4-1110^{9} / \mathrm{L}\right)$, the typical value for $\mathrm{V}_{\max }$ would vary from 4.1 to $4.8 \mathrm{mg} / \mathrm{hr}$, representing a $-6.4 \%$ and $+9.6 \%$ change from the population mean $\mathrm{V}_{\max }$, respectively. The modest change in $V_{\max }$ over the range of normal WBC would not likely necessitate a change in dosing, even in patients who develop leukopenia due to concomitant chemotherapy.

Size covariates are probably the most frequently identified covariates in population PK analyses, and are well established as predictors of systemic exposure for small molecule drugs as well as therapeutic mAbs (13). In this study, three different size covariates (IBW, WGT, and BSA) were evaluated. IBW was found to be a better predictor of $\mathrm{V}_{\max }$ than BSA and WGT. As interactions with EGF- as well as Fc-receptors resulting in receptor-mediated endocytosis are assumed to be the major elimination pathways of cetuximab (137), my results suggest that the number of these receptors in its distribution space is better reflected by IBW than any of the other body size measures. This observation is not unexpected as the elimination capacity of at least the liver and the reticuloendothelial system as major sites of Fc-receptor-mediated elimination of mAbs are only marginally affected by deviations of weight from IBW. The final model predicted a $1.1 \%$ change in $V_{\max }$ per $\mathrm{kg}$ IBW deviation from the median IBW of $64 \mathrm{~kg}$. The estimated changes from the typical population $V_{\max }(4.38 \mathrm{mg} / \mathrm{hr})$ for patients with IBW varying from the observed minimum $(43.3 \mathrm{~kg})$ to the maximum IBW $(81.3 \mathrm{~kg})$ were $-22.4 \%(3.4 \mathrm{mg} / \mathrm{hr})$ and $+18.7 \%(5.2 \mathrm{mg} / \mathrm{hr})$, respectively.

Although $\mathrm{V}_{\max }$ as a direct determinant of cetuximab clearance is best predicted by IBW, clinical dosing of cetuximab is currently performed based on BSA following the traditional standard practice of dosing in oncology (109). To visualize the impact of dosing based on BSA rather than IBW, consider a 6 foot tall male patient whose body 
weight is $77.6 \mathrm{~kg}$, which is also his IBW. Based on BSA calculated according to DuBois (138), his cetuximab maintenance dose would be $498 \mathrm{mg}\left(250 \mathrm{mg} / \mathrm{m}^{2}\right)$. Now suppose the above patient has lost $20 \%$ weight ( $62 \mathrm{~kg}$ body weight; $80 \%$ of IBW) or even $35 \%$ body weight ( $50.4 \mathrm{~kg}$ body weight; $65 \%$ of IBW). His BSA-based cetuximab maintenance dose would be $453 \mathrm{mg}$ (at $62 \mathrm{~kg}$ ) or $415 \mathrm{mg}$ (at $50.4 \mathrm{~kg}$ ), i.e. $9.1 \%$ or $16.7 \%$ lower, but my population PK model would predict the same $\mathrm{V}_{\max }$ for both situations, leading to a reduced systemic exposure of cetuximab in the underweight patient. The modest impact of this effect, however, indicates that the approved BSA-based dosing regimen for cetuximab provides consistent systemic exposure in clinical use even in subjects with substantial deviation between their actual body weight and IBW. Only in very extreme cases of severe underweight, standard dosing might need to be adjusted to avoid underdosing of the patient.

The potential clinical relevance of this finding is supported by the prominence of underweight in patients with advanced stages of solid tumors, including SCCHN. In fact, in the patient population used for this analysis, almost $25 \%$ of the patients weighed less than $80 \%$ of IBW. A comparison of the trough concentrations at 4 weeks and beyond in these patients with the troughs of the other patients in my analysis indicated that the troughs were indeed lower in the underweight group (median 48.2 vs. $62.4 \mu \mathrm{g} / \mathrm{mL}$, $\mathrm{p}=0.014$ ). This finding supports the selection of IBW as predictor for cetuximab elimination rather than BSA or WGT. My results are furthermore in agreement with a previous report that suggests the dosing based on BSA may have limitations for some therapeutic mAbs (45).

Fracasso et al. recently reported a correlation between cetuximab trough levels and antitumor response on cetuximab monotherapy (107). Patients with partial response/stable disease tended to have higher average trough levels compared to those with progressive disease (mean 60.7 vs. $33.2 \mu \mathrm{g} / \mathrm{mL}, \mathrm{p}=0.002$ ). Although these study results were based on a small number of patients $(n=33)$ with different cancer types (predominantly colorectal, breast and head and neck carcinomas), they highlight the potential relevance of adequate systemic exposure, and thus dosing, for achieving antitumor response in cetuximab pharmacotherapy. Further studies, however, are needed to clearly define an optimal systemic exposure range for cetuximab.

The population estimates for $\mathrm{V}_{\max }$ and $\mathrm{K}_{\mathrm{m}}$ are in agreement with findings from other PK analyses of cetuximab $(123,139)$. The PK parameter estimates of my final model are also in line with a previous population analysis of cetuximab PK for regulatory purposes where a two-compartment model with nonlinear elimination was also used (109, 140). This population analysis for regulatory purposes included nineteen clinical studies in various cancer types. The similar results of the two population analyses suggest that cetuximab PK are not different in patients with SCCHN compared with other cancer types. My analysis did not identify an effect of concomitant chemotherapy, cisplatin/carboplatin and 5-fluorouracil, on cetuximab PK which is in agreement with several earlier clinical trials $(81,141)$. Furthermore, I could not detect differences in cetuximab PK for patients receiving cetuximab as first-line treatment during the recurrence or metastatic stage of SCCHN (study A) as compared to patients pretreated 
with at least one line of chemotherapy during this stage (study B). Although several of the tested covariates were not found to influence the PK of cetuximab (e.g., gender, ethnicity, renal and hepatic function), it should be noted that the majority of the studied patients were male, Caucasian, and had adequate hepatic and renal function. Therefore the impact of these covariates on the PK of cetuximab remains to be elucidated.

In summary, I developed a population PK model for cetuximab that characterized the nonlinear PK of this therapeutic mAb. IBW and WBC count were identified as predictors for cetuximab clearance. The currently approved BSA-based dosing of cetuximab provides adequate exposure in most patients, but may require adjustments in cases with extreme deviations of their body weight from their IBW. 


\section{CHAPTER 4. COMPARATIVE PERFORMANCE OF BAYESIAN MARKOV CHAIN MONTE CARLO AND MAXIMUM LIKELIHOOD POPULATION ESTIMATION METHODS WHEN APPLIED TO NONLINEAR PHARMACOKINETICS OF MONOCLONAL ANTIBODIES}

\section{Introduction}

In Chapter 3, a population PK analysis of cetuximab, an anti-EGFR therapeutic $\mathrm{mAb}$, was performed in patients with SCCHN. The final population PK model, which incorporated a two-compartment model with nonlinear elimination, was able to adequately describe the nonlinear PK of cetuximab at the currently approved dosage regimen. During the population PK analysis, various issues were encountered which were likely the result of an attempt to describe this nonlinearity. During evaluation of the different structural PK models, the one- and two-compartment models with nonlinear or parallel linear and nonlinear elimination pathways exhibited convergence difficulties and prolonged run times. The convergence of these PK models was stabilized and run times reduced by log transforming the data and using separate residual error models per clinical study. Limitations with the final population PK model included the inability to quantify between-subject variability simultaneously in $\mathrm{V}_{\max }$ and $\mathrm{K}_{\mathrm{m}}$, the relatively low precision in $\mathrm{K}_{\mathrm{m}}$ compared to other model parameters in the bootstrap analysis (Table 3-2), and the predictive check showed that the model overpredicted the between-subject variability in trough concentrations at week 4 at the lower concentrations (Figure 3-3). Since trough concentrations are largely influenced by $\mathrm{V}_{\max }$, the deviation in the predictive check may have been due to an overestimation of the between-subject variability in $\mathrm{V}_{\max }$.

The difficulty characterizing the nonlinear elimination of cetuximab and the apparent bias in one or more of the parameter estimates were likely due to limitations of the clinical study designs and possibly the population estimation method used. One drawback of the two clinical studies included in the analysis was that dose escalation data was not available as all patients were treated at the same dose level. Another drawback was the unbalanced sampling design of the two studies, as the majority of patients (96 of 143) provided only peak and trough concentrations while the remaining patients provided a full concentration-time profile in addition to peak and trough concentrations. The FOCE method in NONMEM was used throughout the model-building process, and it is known that the first-order approximation methods can be inaccurate when dealing with highly nonlinear models such as the PK model with nonlinear elimination used to describe the PK of cetuximab (112). Therefore, it was questioned whether or not the use of another population estimation method in the cetuximab analysis would have led to more accurate parameter estimates and better characterization of the nonlinear PK of cetuximab (e.g., allowed between-subject variability in $\mathrm{K}_{\mathrm{m}}$ to be estimated).

Nonlinear elimination is a common characteristic of the PK of therapeutic mAbs $(6,8)$, and accordingly, PK models with nonlinear elimination were used in almost half of the population PK analyses of therapeutic mAbs published in the scientific literature (Table 1-1). The question of how well other population estimation methods would have 
performed in evaluating the nonlinear elimination of cetuximab is therefore relevant for other therapeutic mAbs. This question is also relevant for other therapeutic mAbs, because similar difficulties encountered during the cetuximab analysis were also reported in population PK analyses of other therapeutic mAbs. These difficulties included challenges in detecting and characterizing nonlinear elimination due to study design limitations, convergence difficulties with PK models with nonlinear elimination, and the inability to estimate between-subject variability in $\mathrm{K}_{\mathrm{m}}$ (Chapter 1, Table 1-4). It is estimated that half of all current projects in new drug development are biologics, with $\mathrm{mAbs}$ being the predominant class of biologics under clinical study (1). According to a report by Reichert, in 2008 there were over $200 \mathrm{mAbs}$ undergoing clinical study (2). With the growing number of therapeutic mAbs in drug development and reaching FDA approval, the challenges with population PK modeling of therapeutic mAbs with nonlinear PK will continue to remain an issue.

A number of studies have evaluated the performance of different estimation methods for population PK modeling, and in most of these studies a one-compartment model with IV bolus or first-order input and first-order (linear) elimination was used for comparison (142-147). Studies by Hashimoto et al. (148) and Sheiner and Beal (149) evaluated estimation methods in NONMEM for population PK modeling of nonlinear PK data at steady-state. However, both studies used PK models that have not been used in population PK analyses of therapeutic mAbs. Sheiner and Beal used only the MichaelisMenten model where the dosage rate was assumed to be equal to the elimination rate and concentrations were at steady-state. A one-compartment model with nonlinear elimination was used in the analysis by Hashimoto et al. Although Hashimoto et al. used a PK model with nonlinear elimination, in most of the therapeutic mAb population PK analyses a two-compartment model was used (Table 1-1). Bauer et al. recently evaluated various population estimation methods and software when applied to different PK/PD models (150). One of the PK models included in the study was a one-compartment model with parallel linear and nonlinear elimination pathways. While this clearance model has been used in several therapeutic mAb population PK analyses, again in most cases a two-compartment model was used. Also, only one data set was simulated for each PK/PD model in the analysis by Bauer et al., so the population estimation methods could not be systematically evaluated. Therefore, little work has been done evaluating population estimation methods using PK models that are representative of the typical disposition characteristics of therapeutic mAbs.

In the current chapter, a simulation study was conducted to compare the parameter estimation performance of the approximate maximum likelihood methods in NONMEM and a full Bayesian MCMC method in WinBUGS for population PK modeling of therapeutic mAbs with nonlinear PK. A review of the literature in Chapter 1 showed that the population PK of different therapeutic mAbs are quite similar. The findings from published therapeutic $\mathrm{mAb}$ population PK analyses were used in the present study to define aspects of the candidate population PK simulation model in order to simulate PK data for a representative therapeutic mAb. The software NONMEM was included in this study as it is the most widely used software for population PK/PD modeling (151). WinBUGS was chosen as the Bayesian MCMC method provides a 
different approach compared to the estimation methods in NONMEM to solving the population PK problem (152), and there is a growing interest in applying full Bayesian methods in population PK modeling. In addition, the Bayesian MCMC method allowed investigation of the utility of including prior information in a population $\mathrm{PK}$ analysis. The Bayesian MCMC method was evaluated with both uninformative and informative priors, which were obtained from population PK analyses of therapeutic mAbs in the literature. The performance of the different population estimation methods was evaluated under different clinical study designs commonly encountered during drug development, and the sensitivity of estimation error to parameter uncertainty was also explored.

\section{Methods}

\section{Overview of Methodology Steps}

The methodology for this study can be divided into two main steps: simulation and estimation. An overview of the simulation and estimation steps is illustrated in Figure 4-1. Simulation of replicate data sets was performed with uncertainty included simultaneously on all parameters in the population PK model. The software program $\mathrm{R}$ (Version 2.7.2; http://www.r-project.org/) (153) was used to randomly sample 1000 values for each parameter from their respective uncertainty distributions, which were then combined to create 1000 full sets of simulation parameter values for the population PK model. Each full set of simulation parameter values was used to simulate one replicate data set under a given study design template, therefore, 1000 replicate data sets were created per study design. Parameter estimates were then obtained for each replicate using the estimation methods of interest which included three methods in NONMEM (FO, FOCE-I, LAP-I) and a Bayesian MCMC method in WinBUGS. The estimation performance of the methods was evaluated under five different study design scenarios. NONMEM was used to simulate the concentration data sets, and the R package MIfuns (Metrum Institute, Augusta, Maine) (154) was used to automate the creation of replicate NONMEM simulation control streams by systematically substituting in a set of simulation parameter values. The population PK model, study designs, and parameter uncertainty distributions used for performing the simulations are described in the following sections. Example computer codes used for the simulation and estimation steps are provided in Appendix B.

\section{Population Pharmacokinetic Model}

The published findings from population PK analyses of therapeutic mAbs and their general PK behavior (Chapter 1) were used to define the population PK model for this study in terms of model structure and fixed and random effect parameters. For purposes of this study it was assumed that the administered drug was a therapeutic human IgG1 mAb indicated in the area of oncology that targets a cell membrane receptor primarily expressed in tissue. Therapeutic mAbs often exhibit two distinct elimination 


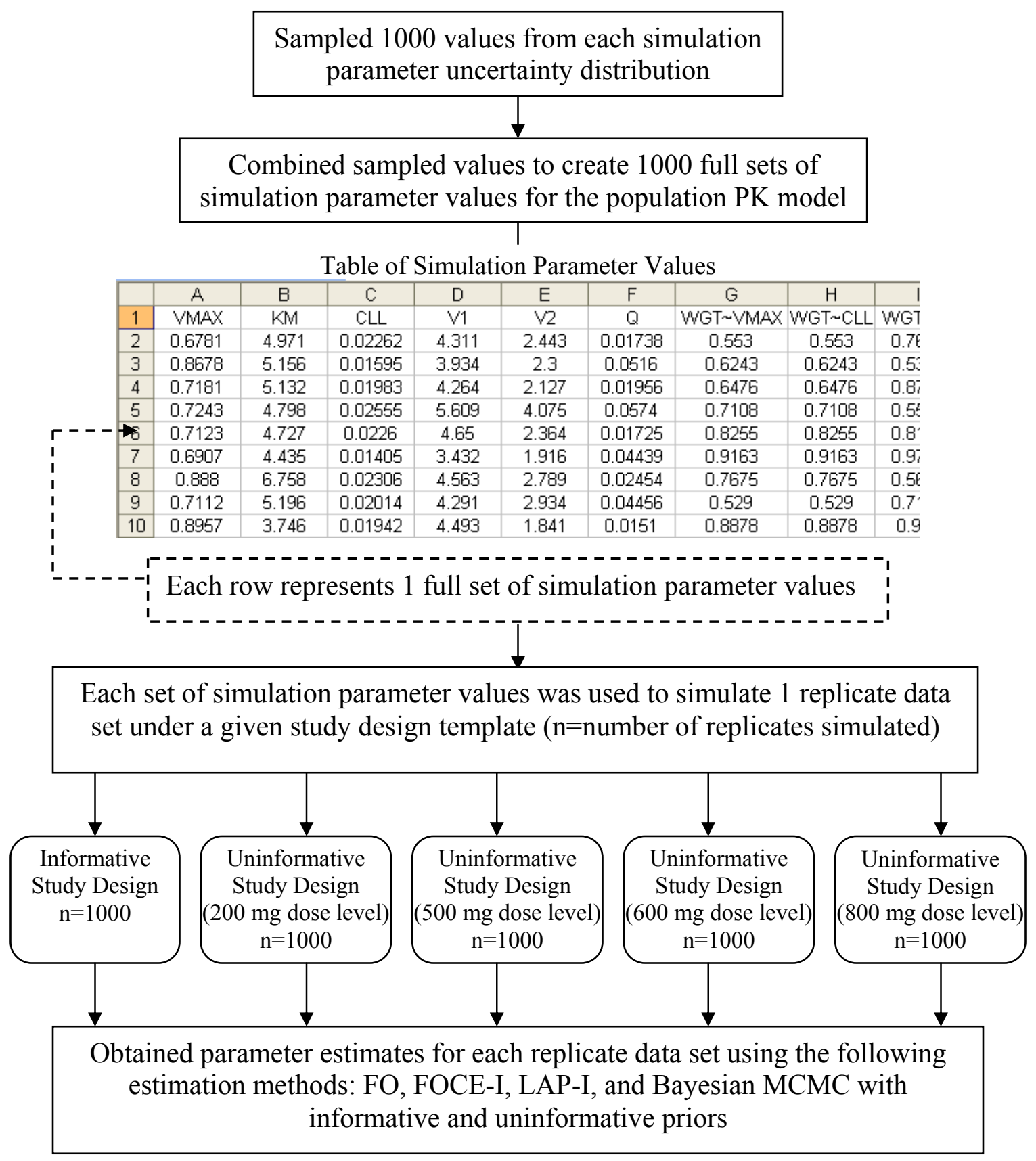

Figure 4-1. Overview of the methodology of the simulation and estimation steps. 
pathways which includes a linear (first-order) clearance pathway mediated by interaction with Fc-receptors and a nonlinear clearance pathway attributable to saturable targetmediated mechanisms (e.g., receptor-mediated endocytosis) (Chapter 1). Therefore, it was believed that the theoretical $\mathrm{mAb}$ for this study was also eliminated via these two pathways. A commonly used PK model in population PK analyses of therapeutic mAbs that can be related to the two distinct physiologic elimination pathways is a twocompartment model with parallel linear and nonlinear elimination from the central compartment (Table 1-1, Figure 1-2A). Accordingly, the same structural PK model was used for the simulation/estimation steps in this study. The use of a two-compartment model was further supported by the fact that a two-compartment model was used in the majority of therapeutic mAb population PK analyses (Table 1-1). The PK model was parameterized in terms of $\mathrm{V}_{\max }, \mathrm{K}_{\mathrm{m}}, \mathrm{CL}_{\mathrm{L}}, \mathrm{V}_{1}, \mathrm{~V}_{2}$, and $\mathrm{Q}$. Under the condition that the absolute bioavailability of the administered dose is known, the parameters for the aforementioned PK model have been shown to be globally identifiable (83). The therapeutic $\mathrm{mAb}$ in this study was administered as an IV infusion so bioavailability was assumed to be 1 . This route of administration was chosen as most therapeutic mAbs that are approved or in development are administered IV (Chapter 1). The parallel elimination PK model was defined in terms of the differential equations below:

$$
\begin{gathered}
\mathrm{C}_{1}=\mathrm{X}_{1} / \mathrm{V}_{1} \\
\mathrm{C}_{2}=\mathrm{X}_{2} / \mathrm{V}_{2} \\
\mathrm{dX} / \mathrm{dt}=-\mathrm{V}_{\text {max }} * \mathrm{C}_{1} /\left(\mathrm{K}_{\mathrm{m}}+\mathrm{C}_{1}\right)-\mathrm{CL}_{\mathrm{L}} * \mathrm{C}_{1}-\mathrm{Q} * \mathrm{C}_{1}+\mathrm{Q} * \mathrm{C}_{2} \\
\mathrm{dX}_{2} / \mathrm{dt}=\mathrm{Q} * \mathrm{C}_{1}-\mathrm{Q} * \mathrm{C}_{2}
\end{gathered}
$$

where $\mathrm{X}_{1}$ and $\mathrm{X}_{2}$ represent the $\mathrm{mAb}$ amount in the central and peripheral compartments, respectively.

The population PK model included between-subject variability for all model parameters except $\mathrm{K}_{\mathrm{m}}$ and $\mathrm{Q}$. Between-subject variability in the parameters $\mathrm{V}_{\max }, \mathrm{CL}_{\mathrm{L}}$, $\mathrm{V}_{1}$, and $\mathrm{V}_{2}$, was assumed to follow a lognormal distribution and was modeled using an exponential error model. The exclusion of between-subject variability for $\mathrm{K}_{\mathrm{m}}$ and $\mathrm{Q}$ in the population PK model is in line with the literature, as between-subject variability in both parameters was often not estimated in population PK analyses of therapeutic mAbs (Tables 1-3 and 1-4). Given that $\mathrm{K}_{\mathrm{m}}$ is related to the affinity of the mAb to its target, it is a reasonable approach to hypothesize that physiologically there is negligible variability in $\mathrm{K}_{\mathrm{m}}$ among individuals. Although between-subject variability in $\mathrm{Q}$ was not modeled, variability in Q can be considered to be intrinsically included in the between-subject variability for $\mathrm{V}_{1}$ and $\mathrm{V}_{2}$ since the volume parameters can be defined in terms of $\mathrm{Q}$ (e.g., $\left.\mathrm{V}_{1}=\mathrm{Q} / \mathrm{K}_{12}\right)$.

Body weight $(\mathrm{kg})$ was included in the population $\mathrm{PK}$ model as a predictor of $\mathrm{V}_{\max }$, $\mathrm{CL}_{\mathrm{L}}, \mathrm{V}_{1}$, and $\mathrm{V}_{2}$. The choice of body weight as a covariate was supported by the observation that body weight was the most commonly identified covariate in therapeutic $\mathrm{mAb}$ population $\mathrm{PK}$ analyses, and it was often found to be a predictor of the 
aforementioned parameters (Table 1-5). The inclusion of a covariate in the model also allowed for evaluating the performance of the estimation methods in estimating a covariate effect. Correlation among the between-subject random effects was modeled using a fixed effect (i.e., weight as a predictor of $\mathrm{V}_{\max }, \mathrm{CL}_{\mathrm{L}}, \mathrm{V}_{1}$, and $\mathrm{V}_{2}$ ) rather than a random effect. Any remaining variability after accounting for the covariate effect was assumed to be uncorrelated. This approach to handling the correlation between the random effects was in part based on the findings from the population PK analyses of sibrotuzumab (45) and matuzumab (40). In the sibrotuzumab analysis, weight was included in the population PK model as a covariate for $\mathrm{V}_{\max }, \mathrm{CL}_{\mathrm{L}}, \mathrm{V}_{1}$, and $\mathrm{V}_{2}$. No correlation was modeled between the random effects for these parameters, although a shared variance term was included in the model between $V_{1}$ and $V_{2}$. In the matuzumab analysis, weight was included in the population PK model as a covariate for $\mathrm{CL}_{\mathrm{L}}$ and $\mathrm{V}_{1}$. Correlation between the random effects was modeled for $V_{1}$ and $V_{2}, V_{\max }$ and $V_{2}$, and $\mathrm{V}_{\max }$ and $\mathrm{V}_{1}$, but no correlation was modeled between $\mathrm{CL}_{\mathrm{L}}$ and $\mathrm{V}_{1}$. The relationship between body weight and the PK parameters was modeled using a power model with the individual patient weight normalized to a weight of $72 \mathrm{~kg}$ as shown in the following equation:

$$
\mathrm{P}=\theta_{1} *\left(\frac{\text { Weight }(\mathrm{kg})}{72 \mathrm{~kg}}\right)^{\theta_{2}}
$$

where the $\theta$ s are the parameters to be estimated, and $\theta_{1}$ represents the typical value of a PK parameter $(\mathrm{P})$ in an individual with a body weight of $72 \mathrm{~kg}$. In most population PK analyses of therapeutic mAbs, the effect of body size (including weight) on a PK parameter was modeled using a power function (Table 1-5).

Residual variability was modeled using an exponential error model. An exponential error model was used to prevent simulation of negative concentrations. Residual variability in most of the therapeutic mAb population PK studies was modeled using either a proportional or a combined additive and proportional error model (Chapter 1). Although an exponential error model was used in this study, the simulation uncertainty distribution for the standard deviation of residual variability (see next section) encompassed a low to moderate range of variability $(\sim 10-20 \% \mathrm{CV})$, and within this range, an exponential error model closely approximates a proportional error model. There were a total of 15 parameters in the population PK model, and the same model was used for both simulation and estimation steps.

\section{Simulation Parameter Uncertainty Distributions}

Simulation of the replicate data sets was performed with uncertainty included simultaneously on all parameters in the population PK model. A review of the literature in Chapter 1 showed that the population PK of different therapeutic mAbs are quite similar. In order to simulate concentration data for a prototypical $\mathrm{mAb}$, the simulation parameter values selected from the uncertainty distributions needed to be realistic and 
representative of the population PK characteristics of therapeutic mAbs. Therefore, the published findings from over 20 population PK analyses of therapeutic mAbs were used to define the simulation parameter uncertainty distributions (Chapter 1, Tables 1-3 through 1-6). In general, a two-step approach was taken in specifying the uncertainty distributions. First, the literature reported parameter values were summarized by calculating summary statistics and creating plots of the parameter values. This information aided in selecting an appropriate type of statistical distribution to describe the parameter uncertainty. After the type of distribution was selected, the second step was to simulate a large number of parameter values from the uncertainty distribution using the summary statistics as initial values for the distribution parameters (e.g., mean and variance). Histograms of the simulated parameter values were created and compared against the literature reported values. The distribution parameters were then adjusted as needed so that the uncertainty distribution adequately reflected the parameter estimates reported in the literature. The approach to specifying the simulation parameter uncertainty distributions was generally to use parameter values reported from population PK analyses of therapeutic mAbs that used a two-compartment model with parallel linear and nonlinear elimination or only nonlinear elimination from the central compartment. However, the final parameter uncertainty distributions were checked visually to make sure that they were representative of parameter values reported from all therapeutic mAb population PK analyses that used a two-compartment PK model regardless of the clearance model. The findings published from population PK analyses that used a onecompartment PK model were not taken into account in specifying the uncertainty distributions.

The approach to specifying the simulation uncertainty distributions for $\mathrm{V}_{\max }$ and $\mathrm{K}_{\mathrm{m}}$ differed from the methodology described above. Population estimates for $\mathrm{V}_{\max }$ and $\mathrm{K}_{\mathrm{m}}$ varied considerably between different therapeutic mAbs (Table 1-4), which can be expected as $\mathrm{V}_{\max }$ and $\mathrm{K}_{\mathrm{m}}$ will be specific for a given $\mathrm{mAb}$ and its pharmacologic target. Therefore, it was not reasonable to use all reported population estimates for $V_{\max }$ and $\mathrm{K}_{\mathrm{m}}$ to specify their respective simulation uncertainty distributions. Instead, the geometric means of the lognormal uncertainty distributions for $\mathrm{V}_{\max }(18 \mathrm{mg} /$ day $)$ and $\mathrm{K}_{\mathrm{m}}(5.0 \mathrm{mg} / \mathrm{L})$ were selected based on reported values from the population PK analysis of matuzumab where a two-compartment model with parallel linear and nonlinear elimination was used (40) (Table 1-4). The population estimates of $\mathrm{V}_{\max }$ and $\mathrm{K}_{\mathrm{m}}$ for matuzumab were 10.9 $\mathrm{mg} /$ day and $4.0 \mathrm{mg} / \mathrm{L}$, respectively. The variances selected for the uncertainty distributions for $\mathrm{V}_{\max }$ and $\mathrm{K}_{\mathrm{m}}$ allowed for a small but reasonable amount of variability in the simulation parameter values, since these parameters are specific for the theoretical $\mathrm{mAb}$ and its target. The expected $\mathrm{CL}_{\text {int }}$ for the theoretical $\mathrm{mAb}$ based on the geometric means of the simulation uncertainty distributions for $\mathrm{V}_{\max }$ and $\mathrm{K}_{\mathrm{m}}$ was $3.6 \mathrm{~L}$ /day, which is in line with other therapeutic mAbs that target cell membrane antigens primarily expressed in tissue (Table 1-4). As discussed in Chapter 1, therapeutic mAbs with cell membrane targets primarily expressed in tissue had similar $\mathrm{CL}_{\text {int }}$ values that were also much smaller compared to mAbs whose cell membrane target was largely expressed on blood cells. 
A lognormal uncertainty distribution was used for most of the parameters in the population PK model since PK parameters often follow this type of distribution. The population values for $\mathrm{V}_{\max }$ and $\mathrm{K}_{\mathrm{m}}$, between-subject standard deviations for $\mathrm{V}_{1}$ and $\mathrm{V}_{2}$, and the standard deviation of residual variability were all randomly sampled from lognormal distributions. The population values for $\mathrm{CL}_{\mathrm{L}}, \mathrm{V}_{1}, \mathrm{~V}_{2}$, and $\mathrm{Q}$ were drawn from a multivariate lognormal distribution. The decision to use a multivariate distribution for $\mathrm{CL}_{\mathrm{L}}, \mathrm{V}_{1}, \mathrm{~V}_{2}$, and $\mathrm{Q}$, and not univariate distributions was based on observed correlations between estimates for these parameters reported in population $\mathrm{PK}$ analyses of $\mathrm{mAbs}$ where a two-compartment parallel elimination model was used (Tables 1-3 and 1-4). The between-subject standard deviations for $\mathrm{V}_{\max }$ and $\mathrm{CL}_{\mathrm{L}}$ and the coefficients for the weight effect were sampled from uniform distributions. A uniform distribution was used for the weight effect on $\mathrm{V}_{\max }, \mathrm{CL}_{\mathrm{L}}, \mathrm{V}_{1}$, and $\mathrm{V}_{2}$, because this type of distribution appeared to describe the estimates reported in the literature for the effect of weight on these parameters better than a lognormal distribution. While a lognormal distribution was considered for the between-subject standard deviations for $\mathrm{V}_{\max }$ and $\mathrm{CL}_{\mathrm{L}}$, a uniform distribution was used because there was interest in evaluating the performance of the estimation methods in cases of low and high between-subject variability in $\mathrm{V}_{\max }$ and $\mathrm{CL}_{\mathrm{L}}$. By using a uniform distribution, this ensured that a larger number of parameter values would be sampled from the extremes of the range specified for the simulation uncertainty distribution. The range of the uncertainty distributions used for the $\mathrm{V}_{\max }$ and $\mathrm{CL}_{\mathrm{L}}$ between-subject standard deviations were based on the minimum and maximum values observed in the literature for the parameters (Table 1-4).

Although the same uncertainty distributions were defined for some parameters (e.g., $\mathrm{V}_{\max }$ and $\mathrm{CL}_{\mathrm{L}}$ between-subject standard deviations), the parameter values were sampled from independent distributions. The exception to this was the coefficient value for the magnitude of the weight effect, which was assumed to be the same for the volume related parameters $\left(\mathrm{V}_{1}\right.$ and $\left.\mathrm{V}_{2}\right)$ and the same for the clearance related parameters $\left(\mathrm{V}_{\max }\right.$ and $\mathrm{CL}_{\mathrm{L}}$ ). The simulation parameter uncertainty distributions are defined below, where $\sim \mathrm{N}\left(\mu, \sigma^{2}\right)$ denotes that the parameter follows a normal distribution with a mean $(\mu)$ and variance $\left(\sigma^{2}\right)$, and $\sim U(a, b)$ refers to a uniform distribution with lower (a) and upper (b) limits. In the case of the multivariate normal distribution, the term $\bar{\mu}$ refers to a vector of means and $\Sigma$ is the variance-covariance matrix.

Fixed Effects

$$
\begin{gathered}
\ln \left(\mathrm{V}_{\max }\right) \sim \mathrm{N}[\ln (18.0 \mathrm{mg} / \text { day }), 0.01] \\
\ln \left(\mathrm{K}_{\mathrm{m}}\right) \sim \mathrm{N}[\ln (5.0 \mathrm{mg} / \mathrm{L}), 0.01] \\
\ln \left(\mathrm{CL}_{\mathrm{L}}, \mathrm{V}_{1}, \mathrm{~V}_{2}, \mathrm{Q}\right) \sim \mathrm{N}(\bar{\mu}, \Sigma)
\end{gathered}
$$




$$
\bar{\mu}=\left[\begin{array}{c}
\ln \left(\mathrm{CL}_{\mathrm{L}}\right) \\
\ln \left(\mathrm{V}_{1}\right) \\
\ln \left(\mathrm{V}_{2}\right) \\
\ln (\mathrm{Q})
\end{array}\right]=\left[\begin{array}{c}
\ln (0.47 \mathrm{~L} / \text { day }) \\
\ln (4.3 \mathrm{~L}) \\
\ln (2.7 \mathrm{~L}) \\
\ln (0.97 \mathrm{~L} / \text { day })
\end{array}\right] \quad \Sigma=\left[\begin{array}{cccc}
0.042 & 0.021 & 0.057 & 0.014 \\
0.021 & 0.019 & 0.039 & 0.045 \\
0.057 & 0.039 & 0.132 & 0.182 \\
0.014 & 0.045 & 0.182 & 0.488
\end{array}\right]
$$

Weight effect on $\mathrm{V}_{\max }$ and $\mathrm{CL}_{\mathrm{L}} \sim \mathrm{U}(0.4,1.0)$

Weight effect on $\mathrm{V}_{1}$ and $\mathrm{V}_{2} \sim \mathrm{U}(0.4,1.0)$

Between-Subject Variability

(Distributions defined for standard deviation)

$$
\begin{array}{lr}
\mathrm{V}_{\max } \omega \sim \mathrm{U}(0.15,0.65) & \mathrm{CL}_{\mathrm{L}} \omega \sim \mathrm{U}(0.15,0.65) \\
\ln \left(\mathrm{V}_{1} \omega\right) \sim \mathrm{N}[\ln (0.25), 0.04] & \ln \left(\mathrm{V}_{2} \omega\right) \sim \mathrm{N}[\ln (0.25), 0.04]
\end{array}
$$

Residual Variability

(Distribution defined for standard deviation)

$$
\ln (\sigma) \sim \mathrm{N}[\ln (0.15), 0.04]
$$

Individual patient weights were simulated simultaneously with concentration data for each replicate data set, since weight was included in the population PK model as a covariate. Given that the $\mathrm{mAb}$ in this study was assumed to be for therapeutic use in the area of oncology, the goal was to simulate patient weights that were representative of a population of adult cancer patients. Patient weights were assumed to follow a lognormal distribution with a variance of 0.04 and a mean weight that was randomly sampled from the following uncertainty distribution:

$$
\ln (\text { population mean weight }) \sim \mathrm{N}\left[\ln (72 \mathrm{~kg}), 6.25 \mathrm{E}^{-4}\right]
$$

Therefore, the mean patient weight was different for each replicate data set, but the variability in patient weights remained the same. Specification of the uncertainty distribution for the mean patient weight was based on median weights reported in population PK analyses of therapeutic mAbs in cancer patients (Table 1-1). The decision to use 0.04 as the between-subject variance for patient weight was based on the variability of patient weights in the cetuximab population PK analysis (Chapter 3). In addition, patient weights were available from a study by Sparreboom et al. (personal communication) where the PK of different anticancer drugs was evaluated in over 1,200 adult cancer patients (155). This data was also taken into account when specifying the variability for patient weights and the uncertainty distribution for the mean patient weight. 


\section{Study Designs}

Replicate data sets were simulated under two different study designs typically encountered during drug development: a dose-ranging design ('informative design') and a single dose level design ('uninformative design'). The study templates were designed to be representative of what one might see in typical phase I/II studies of therapeutic $\mathrm{mAbs}$ in drug development. The study designs used herein were similar in nature to a number of clinical studies included in the population PK analyses of various therapeutic mAbs $(21,23,29,30,40,45)$.

In the informative design there were six dose groups with six patients per group. The therapeutic mAb was administered weekly for 4 weeks as a $1 \mathrm{hr}$ IV infusion at doses of 50,100, 200,400,800, or $1600 \mathrm{mg}$. Peak and trough mAb concentrations were obtained at weeks 2 and 3, and on weeks 1 and 4 a full PK concentration-time profile was obtained with concentrations sampled at 1, 3, 6, 10, 24, 48, 72, 96, and 168 hours after the start of the infusion. This sampling schedule resulted in 22 data points per patient. The selection of the dose levels was based on simulations of concentrations at the sampling time points using the means and midpoints of the simulation uncertainty distributions. The dose levels for the informative study design were selected because they resulted in concentration ranges that were below and above the $\mathrm{K}_{\mathrm{m}}$. The software program PopED (Version 2.06; http://poped.sourceforge.net/) (156) was also used to aid in the selection of dose levels for the informative design. Attempts were made to simultaneously optimize dose levels and sampling times using PopED, but this was not feasible due to prolonged run times, so only the dose levels were optimized. Given the similarities in population PK between the theoretical $\mathrm{mAb}$ for this study and matuzumab (including population estimates for $\mathrm{V}_{\max }$ and $\mathrm{K}_{\mathrm{m}}$ ), the sampling times reported for three phase I trials included in the population PK analysis of matuzumab (40) were taken into account in selecting the sampling times for the informative (and uninformative) study design.

In the uninformative design there were 36 patients and all were treated at the same dose level. There were 4 different versions of the uninformative design with patients treated at different dose levels of $200,500,600$, or $800 \mathrm{mg}$. Just as with the informative design, simulations were used to help select dose levels for the uninformative design. The dose levels were selected so that the concentration ranges would either be below/around (200 $\mathrm{mg}$ ) or above $\left(500,600\right.$, and $800 \mathrm{mg}$ ) the $\mathrm{K}_{\mathrm{m}}$, thereby making it more difficult to detect and characterize the nonlinear elimination of the $\mathrm{mAb}$. The dosing and sampling schedules were the same as in the informative design except for the full PK concentration-time profile at week 1 which was removed, resulting in 13 data points per patient.

\section{Estimation Methods}

The estimation methods evaluated in this study included approximate maximum likelihood methods available in NONMEM and a Bayesian MCMC method with two 
different sets of priors (informative and uninformative). The estimation methods evaluated in NONMEM (Version VI, level 2.0; Icon Development Solutions, Ellicott City, Maryland) (20) were FO, FOCE-I, and LAP-I. Computer resources included a computer grid, with each node running NetBSD 3.1 (64bit) on AMD64 processors, and the GNU Fortran compiler GCC-3.3.3 (GNU Project; http://www.gnu.org/). NMQual 6.3 (Metrum Institute, Augusta, Maine; http://code.google.com/p/nmqual/) was used to track all code patches/options and install the NONMEM software. Simulation of the replicate data sets was also carried out using NONMEM and the computer grid. The twocompartment parallel elimination PK model was implemented in NONMEM using the subroutine ADVAN6 TRANS1 TOL $=8$. The default boundary test in NONMEM was disabled for all parameters in the population PK model by specifying the NOTHETABOUNDTEST, NOOMEGABOUNDTEST, and NOSIGMABOUNDTEST options in the \$ESTIMATION record. If an estimation run crashed or minimization terminated, up to four restarts were allowed in which the TOL or initial estimates were adjusted according to a predefined protocol. All runs that reported parameter estimates, including those where minimization terminated, were used in the analysis. The initial estimates used for NONMEM are shown below, and for most parameters the initial estimates were within $20-100 \%$ of the mean or the midpoint of their respective simulation parameter uncertainty distribution:

\section{Fixed Effects}

$$
\begin{aligned}
& \mathrm{V}_{\max }=28.8 \mathrm{mg} / \text { day } \\
& \mathrm{K}_{\mathrm{m}}=10.0 \mathrm{mg} / \mathrm{L} \\
& \mathrm{CL}_{\mathrm{L}}=0.72 \mathrm{~L} / \text { day } \\
& \mathrm{V}_{1}=3.0 \mathrm{~L} \\
& \mathrm{~V}_{2}=2.0 \mathrm{~L} \\
& \mathrm{Q}=1.8 \mathrm{~L} / \text { day } \\
& \text { Weight } \sim \mathrm{V}_{\max }=0.8 \\
& \text { Weight } \sim \mathrm{CL}_{\mathrm{L}}=0.8 \\
& \text { Weight } \sim \mathrm{V}_{1}=0.8 \\
& \text { Weight } \sim \mathrm{V}_{2}=0.8 \\
& \mathrm{~V}_{\max } \omega=0.4 \\
& \mathrm{CL}_{\mathrm{L}} \omega=0.4 \\
& \mathrm{~V}_{1} \omega=0.4 \\
& \mathrm{~V}_{2} \omega=0.4 \\
& \sigma=0.4
\end{aligned}
$$

The population PK model was also implemented in WinBUGS (version 1.4.3; http://www.mrc-bsu.cam.ac.uk/bugs/) (114) using BUGSModelLibrary (version 1.1; 
Metrum Institute, Augusta, Maine; http://code.google.com/p/bugsmodellibrary/) (157), a prototype PK/PD model library developed for use with WinBUGS. The use of BUGSModelLibrary also required the installation of WBDev (http://www.winbugsdevelopment.org.uk/wbdev.html) (158) and the BlackBox Component Builder (version 1.5; Oberon Microsystems, Zurich, Switzerland; http://www.oberon.ch/blackbox.html). Data management, launching WinBUGS, and analysis of the MCMC samples was done using R with the R packages coda (159) and a modified version of R2WinBUGS (160) contributed by W.R. Gillespie (Metrum Research Group, Tariffville, CT). WinBUGS was run on a Thinkmate VSX i20S4-T with 2 Intel quad-core Xeon $53452.33 \mathrm{GHz}$ processors and 16 GB RAM. The operating system was Microsoft Windows Server 2003.

The Bayesian MCMC method was evaluated with uninformative (vague) priors. The rationale behind specifying the uninformative priors is as follows. For $V_{\max }, \mathrm{K}_{\mathrm{m}}$, $\mathrm{CL}_{\mathrm{L}}, \mathrm{V}_{1}, \mathrm{~V}_{2}$, and $\mathrm{Q}$, the geometric means of their respective uninformative priors were set to the initial estimates used for NONMEM. By assuming a lognormal prior distribution for these parameters and using a variance of $10,000(\mathrm{SD}=100)$, the priors can be considered vague since the $95 \%$ confidence interval would be approximately 0 and $+\infty$ on the linear scale. Uniform priors were specified for the weight effects, which is the same type of distribution used for their simulation parameter values, but the range of the uniform priors was increased to 0 to 5 . The range in the case was constrained to be positive, as one would not expect weight to be a negative predictor of clearance and volume parameters. Although some informativeness was imparted in the priors by doing this, the range of the uniform distributions was large enough to be considered weakly informative. Uniform priors with a large range of $1.0 \mathrm{E}^{-4}$ to $1.0 \mathrm{E}^{4}$ were specified for the standard deviations of between-subject variability and residual variability. The uninformative priors for the random effects were chosen based on recommendations from a paper by A. Gelman in which various uninformative prior distributions for variance parameters in hierarchical models are discussed (161).

The Bayesian MCMC method was also evaluated with informative priors. A review of the literature in Chapter 1 indicated that many characteristics of the population PK of currently used therapeutic mAbs are quite similar. Therefore, the idea was that the published findings from the population PK analyses of therapeutic mAbs could be used as prior information for the population model parameters. The rationale and methodology used to specify the informative priors was similar to that used for the simulation parameter uncertainty distributions. Although the literature was used in specifying both the informative priors and simulation parameter uncertainty distributions, the distribution parameter values for the informative priors differed somewhat from the simulation uncertainty distributions, as the priors were updated with additional therapeutic $\mathrm{mAb}$ population PK studies that were published or found in the literature from the time the simulation uncertainty distributions were specified. The types of distributions used for the model parameters were the same for the informative priors and the simulation uncertainty distributions. The exception to this was the between-subject standard deviation for $\mathrm{V}_{\max }$ and $\mathrm{CL}_{\mathrm{L}}$, where lognormal priors were used since the reasoning behind using a uniform distribution for the simulation uncertainty distributions no longer applied for the estimation step. Although a Wishart distribution is commonly 
used in Bayesian modeling as a prior for the variance-covariance matrix of the betweensubject random effects, in this case, lognormal distributions were used since it was assumed that between-subject variability was uncorrelated to keep consistent with the assumptions made for the simulation model.

As previously mentioned, the population estimates for $\mathrm{V}_{\max }$ and $\mathrm{K}_{\mathrm{m}}$ varied considerably between different therapeutic mAbs (Table 1-4). This can be expected as $\mathrm{V}_{\max }$ and $\mathrm{K}_{\mathrm{m}}$ will be specific for a given $\mathrm{mAb}$ and its pharmacologic target. Therefore, it was not plausible to use reported population estimates for $\mathrm{V}_{\max }$ and $\mathrm{K}_{\mathrm{m}}$ as prior information. However, in Chapter 1 it was observed that therapeutic mAbs with cell membrane targets primarily expressed in tissue had similar $\mathrm{CL}_{\text {int }}$ values that were also much smaller compared to mAbs whose cell membrane target was largely expressed on blood cells. In the current study, it was assumed that the theoretical mAb targets a cell membrane receptor primarily expressed in tissue. Thus, the $\mathrm{CL}_{\text {int }}$ values (Table 1-4) for other therapeutic mAbs (e.g., sibrotuzumab, matuzumab, cetuximab, and CP-751,871) that also bind to cell membrane antigens primarily expressed in the tissue were used as prior information for the $\mathrm{CL}_{\text {int }}$ of the theoretical $\mathrm{mAb}$. In addition to $\mathrm{CL}_{\text {int }}$, an informative prior was specified for $\mathrm{K}_{\mathrm{m}}$, and then $\mathrm{V}_{\max }$ was determined as a secondary parameter as $\mathrm{CL}_{\text {int }} * \mathrm{~K}_{\mathrm{m}}$. In order to specify an informative prior for $\mathrm{K}_{\mathrm{m}}$, an assumption had to be made that prior information was available during drug development of the theoretical $\mathrm{mAb}$. It was assumed that a single dose, dose escalation, first-in-man study of the theoretical $\mathrm{mAb}$ had been conducted, and $\mathrm{K}_{\mathrm{m}}$ was estimated from a standard twostage PK analysis of the concentration data (a population PK analysis could have been conducted as well). In addition, $\mathrm{K}_{\mathrm{m}}$ may have been estimated in pre-clinical PK studies in animals, and this information could be used in the prior for $\mathrm{K}_{\mathrm{m}}$. During drug development, in vitro binding studies are commonly performed for the $\mathrm{mAb}$ and its pharmacologic target, and this could be used as prior information for $\mathrm{K}_{\mathrm{m}}$ although the $\mathrm{Kd}$ and $\mathrm{K}_{\mathrm{m}}$ are often not similar. A lognormal prior was used for $\mathrm{K}_{\mathrm{m}}$ with a geometric mean of $10 \mathrm{mg} / \mathrm{L}$ and a variance of 1.38 , which resulted in a $95 \%$ confidence interval of approximately 1 and $100 \mathrm{mg} / \mathrm{L}$ on the linear scale. This seemed like a reasonable degree of informativeness for $\mathrm{K}_{\mathrm{m}}$ given the realistic availability of prior information from the previously mentioned scenarios.

In WinBUGS, the variability of a normal distribution is defined in terms of precision (inverse of variance), or in the case of a multivariate normal distribution a precision matrix (inverse of covariance matrix) is used. However, for the uninformative and informative prior distributions described below, the uncertainty is defined in terms of variance or a covariance matrix so that comparisons can be easily made with the simulation parameter uncertainty distributions. The notations used for the simulation uncertainty distributions also apply for the prior distributions.

\section{$\underline{\text { Uninformative Priors }}$}

Fixed Effects

$$
\ln \left(\mathrm{V}_{\max }\right) \sim \mathrm{N}[\ln (28.8 \mathrm{mg} / \text { day }), 10000] \quad \ln \left(\mathrm{K}_{\mathrm{m}}\right) \sim \mathrm{N}[\ln (10.0 \mathrm{mg} / \mathrm{L}), 10000]
$$




$$
\begin{array}{lr}
\ln \left(\mathrm{CL}_{\mathrm{L}}\right) \sim \mathrm{N}[\ln (0.72 \mathrm{~L} / \text { day }), 10000] & \ln \left(\mathrm{V}_{1}\right) \sim \mathrm{N}[\ln (3.0 \mathrm{~L}), 10000] \\
\ln \left(\mathrm{V}_{2}\right) \sim \mathrm{N}[\ln (2.0 \mathrm{~L}), 10000] & \ln (\mathrm{Q}) \sim \mathrm{N}[\ln (1.8 \mathrm{~L} / \text { day }), 10000] \\
\text { Weight effect on } \mathrm{V}_{\max } \sim \mathrm{U}(0,5.0) & \text { Weight effect on } \mathrm{CL}_{\mathrm{L}} \sim \mathrm{U}(0,5.0) \\
\text { Weight effect on } \mathrm{V}_{1} \sim \mathrm{U}(0,5.0) & \text { Weight effect on } \mathrm{V}_{2} \sim \mathrm{U}(0,5.0) \\
\text { Between-Subject Variability } \\
(\text { Distributions defined for standard deviation }) \\
\mathrm{V}_{\max } \omega \sim \mathrm{U}\left(1.0 \mathrm{E}^{-4}, 1.0 \mathrm{E}^{4}\right) & \mathrm{CL}_{\mathrm{L}} \omega \sim \mathrm{U}\left(1.0 \mathrm{E}^{-4}, 1.0 \mathrm{E}^{4}\right) \\
\mathrm{V}_{1} \omega \sim \mathrm{U}\left(1.0 \mathrm{E}^{-4}, 1.0 \mathrm{E}^{4}\right) & \mathrm{V}_{2} \omega \sim \mathrm{U}\left(1.0 \mathrm{E}^{-4}, 1.0 \mathrm{E}^{4}\right)
\end{array}
$$

Residual Variability

(Distribution defined for standard deviation)

$$
\sigma \sim \mathrm{U}\left(1.0 \mathrm{E}^{-4}, 1.0 \mathrm{E}^{4}\right)
$$

Informative Priors

\section{Fixed Effects}

$$
\begin{aligned}
& \ln \left(\mathrm{CL}_{\text {int }}\right) \sim \mathrm{N}[\ln (2.4 \mathrm{~L} / \text { day }), 0.25] \quad \ln \left(\mathrm{K}_{\mathrm{m}}\right) \sim \mathrm{N}[\ln (10.0 \mathrm{mg} / \mathrm{L}), 1.38] \\
& \ln \left(\mathrm{CL}_{\mathrm{L}}, \mathrm{V}_{1}, \mathrm{~V}_{2}, \mathrm{Q}\right) \sim \mathrm{N}(\bar{\mu}, \Sigma) \\
& \bar{\mu}=\left[\begin{array}{c}
\ln \left(\mathrm{CL}_{\mathrm{L}}\right) \\
\ln \left(\mathrm{V}_{1}\right) \\
\ln \left(\mathrm{V}_{2}\right) \\
\ln (\mathrm{Q})
\end{array}\right]=\left[\begin{array}{c}
\ln (0.25 \mathrm{~L} / \text { day }) \\
\ln (3.6 \mathrm{~L}) \\
\ln (2.6 \mathrm{~L}) \\
\ln (0.80 \mathrm{~L} / \text { day })
\end{array}\right] \quad \Sigma=\left[\begin{array}{cccc}
0.855 & 0.266 & 0.305 & 0.234 \\
0.266 & 0.090 & 0.125 & 0.089 \\
0.305 & 0.125 & 0.349 & 0.140 \\
0.234 & 0.089 & 0.140 & 0.317
\end{array}\right]
\end{aligned}
$$

Weight effect on $\mathrm{V}_{\max } \sim \mathrm{U}(0.25,1.25) \quad$ Weight effect on $\mathrm{CL}_{\mathrm{L}} \sim \mathrm{U}(0.25,1.25)$

Weight effect on $\mathrm{V}_{1} \sim \mathrm{U}(0.25,1.25) \quad$ Weight effect on $\mathrm{V}_{2} \sim \mathrm{U}(0.25,1.25)$ 
Between-Subject Variability

(Distributions defined for standard deviation)

$$
\begin{array}{lr}
\ln \left(\mathrm{V}_{\max } \omega\right) \sim \mathrm{N}[\ln (0.35), 0.16] & \ln \left(\mathrm{CL}_{\mathrm{L}} \omega\right) \sim \mathrm{N}[\ln (0.35), 0.16] \\
\ln \left(\mathrm{V}_{1} \omega\right) \sim \mathrm{N}[\ln (0.25), 0.16] & \ln \left(\mathrm{V}_{2} \omega\right) \sim \mathrm{N}[\ln (0.25), 0.16]
\end{array}
$$

Residual Variability

(Distribution defined for standard deviation)

$$
\ln (\sigma) \sim \mathrm{N}[\ln (0.20), 0.16]
$$

Due to prolonged run times, WinBUGS was only evaluated under the informative and uninformative $600 \mathrm{mg}$ dose level study designs, and the number of estimation runs was limited to 100 replicate data sets. WinBUGS was run with three MCMC chains for 250,000 iterations with a burn-in phase of 25,000 and every $25^{\text {th }}$ sample was retained. Therefore, a total of 27,000 MCMC samples were retained for analysis. The length of the burn-in phase was determined by performing a number of trial runs $(n=15)$ with different simulated replicate data sets (under both the informative and uninformative $600 \mathrm{mg}$ study designs), and convergence was monitored by visualization of the MCMC chain histories and the use of Gelman-Rubin-Brooks plots (162). The trial runs were also performed with both uninformative and informative priors. Since the data sets were different for each Bayesian MCMC run, a conservative number was chosen for the burn-in phase to increase the likelihood that convergence had been reached in all runs. The trial runs were also used to determine an adequate number of iterations for sampling after the burn-in phase. For purposes of this study, it was believed that 250,000 iterations for each MCMC chain would be an adequate number to sufficiently characterize the posterior median, which was chosen as the summary statistic of interest to calculate the parameter bias and precision. For example, had the 2.5 and 97.5 percentiles of the posterior distribution been of interest, then it is likely that more MCMC samples would be needed to characterize the tails of the posterior distribution. An adequate number of MCMC samples after the burnin phase was also assessed by using the Monte Carlo error, which is similar to the standard error of the mean but adjusted for autocorrelation between the parameters. A Monte Carlo error of less than 5\% of the standard deviation of the MCMC samples was considered to be sufficient for this study.

The BUGSModelLibrary offers a choice of two different ordinary differential equation solvers, and the Runge-Kutta $4^{\text {th }} / 5^{\text {th }}$ order method was used for this analysis. Initial estimates for the three MCMC chains were interdispersed by $25 \%$. When uninformative priors were used, the initial estimates for one of the MCMC chains were set equal to those used for NONMEM. When informative priors were used, the initial estimates for one of the MCMC chains were set equal to the means and midpoints of the informative prior distributions, which were comparable to the initial estimates used for NONMEM. 


\section{Evaluation of Estimation Performance}

The estimation results from NONMEM differ from WinBUGS, as NONMEM provides maximum likelihood estimates while WinBUGS provides full posterior probability distributions of the parameters. In order to make comparisons between NONMEM and WinBUGS with regards to estimation performance, the median of the posterior distributions was chosen as the point estimate. The parameter values used to simulate each replicate data set were considered to be the true parameter values. The difference between the parameter estimate and true value was calculated as the percent estimation error:

$$
\text { Percent Estimation Error }=\frac{\left(\text { Parameter }_{\text {Estimate }}-\text { Parameter }_{\text {True }}\right)}{\text { Parameter }_{\text {True }}} \times 100
$$

The percent estimation errors were summarized for each estimation method under a given study design by calculating bias and precision. Bias was calculated as the median percent estimation error (MPE), and precision was calculated as the median absolute percent estimation error (MAPE):

$$
\begin{array}{r}
\text { MPE }=\operatorname{Median}\left[\frac{\left(\text { Parameter }_{\text {Estimate }}-\text { Parameter }_{\text {True }}\right)}{\text { Parameter }_{\text {True }}} \times 100\right] \\
\text { MAPE }=\text { Median }\left[\text { Absolute value }\left(\frac{\left(\text { Parameter }_{\text {Estimate }}-\text { Parameter }_{\text {True }}\right)}{\text { Parameter }_{\text {True }}} \times 100\right)\right]
\end{array}
$$

A global sensitivity analysis was performed to evaluate the dependence of simulation conclusions on parameter uncertainty. The sensitivity analysis was visualized by plotting the percent estimation error vs. the specific value of simulation model parameters for each trial replicate. The plots were then viewed for trends in the estimation error when viewed across the range of parameter uncertainty employed for the simulations. These sensitivity plots were created for each simulation model parameter.

Predictive checks were performed to assess whether the observed parameter biases would result in predictions that deviate significantly from the "true model". For purposes of the predictive check, the means and midpoints of the simulation parameter uncertainty distributions were assumed to be the true parameter values. The true parameter values were then altered based on the parameter biases observed in the analysis. Monte Carlo simulations were performed with both the true and biased parameter values using a template data set from one of the study designs. The median and $90 \%$ prediction interval of simulated concentrations during the week 4 full concentration-time profile served as the metrics by which the true model predictions and analysis-derived predictions were compared. 


\section{Results}

Under all study designs, the FO method generally produced larger bias and lower precision for the model parameters compared to the other estimation methods. Therefore, the following discussion of the results is based on the findings for the FOCE-I, LAP-I, and Bayesian MCMC methods. The discussion is also focused on the results from the informative and uninformative $600 \mathrm{mg}$ dose level study designs, as these were the two designs under which both NONMEM and WinBUGS were evaluated and could be compared. Results for the NONMEM estimation methods under the uninformative 200, 500, and $800 \mathrm{mg}$ dose level study designs can be found in Appendix C (Tables C-1 and $\mathrm{C}-2)$. MCMC chain histories, probability densities of the posterior distributions of the model parameters, and Gelman-Rubin-Brooks plots for representative estimation runs using both sets of Bayesian priors under the informative and uninformative $600 \mathrm{mg}$ dose designs are also presented in Appendix C (Figures C-1 through C-3).

\section{Informative Study Design}

Bias and precision of the population PK model parameters under the informative study design are summarized in Tables 4-1 and 4-2, and boxplots of percent estimation errors of the model parameters are shown in Figure 4-2. It should be noted that for the random effect parameters (i.e., between-subject variability and residual variability) bias and precision was calculated with respect to the variance term. No appreciable bias was observed for the estimation methods, as the MPE was less than $\pm 25 \%$ (ranged from $-24 \%$ to $21 \%$ ) for all parameters. Bias was comparable across the estimation methods for a given parameter, with the most noticeable difference being the smaller bias for the $\mathrm{V}_{2}$ between-subject variance when uninformative or informative Bayesian priors were used compared to FOCE-I and LAP-I. For each estimation method, parameter bias tended to be larger for the between-subject variances compared to other model parameters.

Good precision was observed for the estimation methods, as the MAPE was less than $52 \%$ (ranged from $3 \%$ to $52 \%$ ) for all model parameters. Under each estimation method, the degree of precision varied among the parameters as a lower precision (i.e., larger MAPE) was observed for the covariate effects and between-subject variances compared to other parameters in the model. Precision was similar across the estimation methods for a given parameter, but Bayesian MCMC (particularly with informative priors) produced noticeably better precision in the covariate effects and the $\mathrm{V}_{2}$ betweensubject variance compared to FOCE-I and LAP-I. The differences in parameter precision between the estimation methods were also evident in the boxplots in Figure 4-2 for the aforementioned parameters.

Bias and precision of the PK parameter estimates for Bayesian MCMC with uninformative priors were comparable to that when informative priors were used, which can be expected given the informativeness of the data under the dose escalation study design. 
Table 4-1. Bias (median percent estimation error) in the population pharmacokinetic model parameters under the informative study design.

\begin{tabular}{|c|c|c|c|c|c|}
\hline Parameter & FO & FOCE-I & LAP-I & $\begin{array}{l}\text { B-MCMC } \\
\text { [UP] }\end{array}$ & $\begin{array}{l}\text { B-MCMC } \\
{[\mathrm{IP}]}\end{array}$ \\
\hline $\mathrm{V}_{\max }$ & -3.6 & 4.5 & 0.67 & -5.6 & -3.0 \\
\hline $\mathrm{K}_{\mathrm{m}}$ & 17 & 2.9 & 1.3 & -1.1 & 1.2 \\
\hline $\mathrm{CL}_{\mathrm{L}}$ & 0.12 & -0.61 & -2.2 & 0.46 & -0.062 \\
\hline $\mathrm{V}_{1}$ & -2.8 & -0.83 & -1.2 & 0.32 & 0.062 \\
\hline $\mathrm{V}_{2}$ & 21 & -0.46 & -1.3 & 3.2 & 2.3 \\
\hline Q & 17 & -1.5 & -0.79 & -0.25 & -0.50 \\
\hline $\mathrm{WGT} \sim \mathrm{V}_{\max }$ & -0.59 & -0.32 & -0.037 & 8.7 & 1.4 \\
\hline $\mathrm{WGT} \sim \mathrm{CL}_{\mathrm{L}}$ & -3.8 & 0.47 & -0.024 & 1.5 & 6.9 \\
\hline $\mathrm{WGT} \sim \mathrm{V}_{1}$ & 0.027 & 1.5 & 1.4 & 4.3 & 3.5 \\
\hline $\mathrm{WGT} \sim \mathrm{V}_{2}$ & -0.77 & -1.3 & -1.3 & 11 & 5.4 \\
\hline$V_{\max } \operatorname{BSV}\left(\omega^{2}\right)$ & -30 & -8.1 & -8.4 & 21 & 1.4 \\
\hline $\mathrm{CL}_{L} \operatorname{BSV}\left(\omega^{2}\right)$ & 18 & -13 & -12 & 4.3 & -9.5 \\
\hline $\mathrm{V}_{1} \operatorname{BSV}\left(\omega^{2}\right)$ & -4.6 & -8.6 & -7.8 & 1.5 & -2.3 \\
\hline $\mathrm{V}_{2} \operatorname{BSV}\left(\omega^{2}\right)$ & 121 & -18 & -24 & 2.8 & -0.59 \\
\hline Residual variance $\left(\sigma^{2}\right)$ & 16 & -0.65 & -0.76 & 0.82 & 1.3 \\
\hline
\end{tabular}

B-MCMC = Bayesian Markov Chain Monte Carlo; BSV = between-subject variance; $\mathbf{I P}=$ informative priors; $\mathbf{U P}=$ uninformative priors; WGT = weight. 
Table 4-2. Precision (median absolute percent estimation error) in the population pharmacokinetic model parameters under the informative study design.

\begin{tabular}{|c|c|c|c|c|c|}
\hline Parameter & FO & FOCE-I & LAP-I & $\begin{array}{l}\text { B-MCMC } \\
\text { [UP] }\end{array}$ & $\begin{array}{l}\text { B-MCMC } \\
\text { [IP] }\end{array}$ \\
\hline $\mathrm{V}_{\max }$ & 19 & 11 & 11 & 11 & 9.5 \\
\hline $\mathrm{K}_{\mathrm{m}}$ & 32 & 11 & 11 & 9.4 & 8.8 \\
\hline $\mathrm{CL}_{\mathrm{L}}$ & 11 & 7.9 & 8.6 & 9.5 & 7.8 \\
\hline $\mathrm{V}_{1}$ & 3.7 & 2.9 & 3.1 & 3.2 & 2.7 \\
\hline $\mathrm{V}_{2}$ & 22 & 5.7 & 6.0 & 7.2 & 6.4 \\
\hline Q & 17 & 5.2 & 5.5 & 5.0 & 4.7 \\
\hline $\mathrm{WGT} \sim \mathrm{V}_{\max }$ & 88 & 47 & 49 & 28 & 14 \\
\hline $\mathrm{WGT} \sim \mathrm{CL}_{\mathrm{L}}$ & 77 & 51 & 52 & 40 & 20 \\
\hline $\mathrm{WGT} \sim \mathrm{V}_{1}$ & 26 & 21 & 21 & 21 & 16 \\
\hline $\mathrm{WGT} \sim \mathrm{V}_{2}$ & 61 & 37 & 39 & 30 & 15 \\
\hline $\mathrm{V}_{\max } \operatorname{BSV}\left(\omega^{2}\right)$ & 43 & 25 & 25 & 37 & 25 \\
\hline $\mathrm{CL}_{L} \operatorname{BSV}\left(\omega^{2}\right)$ & 39 & 27 & 26 & 30 & 23 \\
\hline $\mathrm{V}_{1} \operatorname{BSV}\left(\omega^{2}\right)$ & 19 & 17 & 18 & 19 & 17 \\
\hline $\mathrm{V}_{2} \operatorname{BSV}\left(\omega^{2}\right)$ & 121 & 48 & 49 & 43 & 28 \\
\hline Residual variance $\left(\sigma^{2}\right)$ & 16 & 4.0 & 4.0 & 3.8 & 3.3 \\
\hline
\end{tabular}

B-MCMC = Bayesian Markov Chain Monte Carlo; BSV = between-subject variance; $\mathbf{I P}=$ informative priors; $\mathbf{U P}=$ uninformative priors; WGT = weight. 
Figure 4-2. Boxplots of percent estimation errors for the population pharmacokinetic model parameters under the informative study design. Outliers are not shown for visualization purposes and made up $<10 \%$ of the data for each parameter-estimation method combination. The median percent estimation error corresponds to the calculated parameter bias. Note that the y-axis scale differs from one boxplot to another. BMCMC = Bayesian Markov Chain Monte Carlo; IP = informative priors; $\mathbf{U P}=$ uninformative priors. 

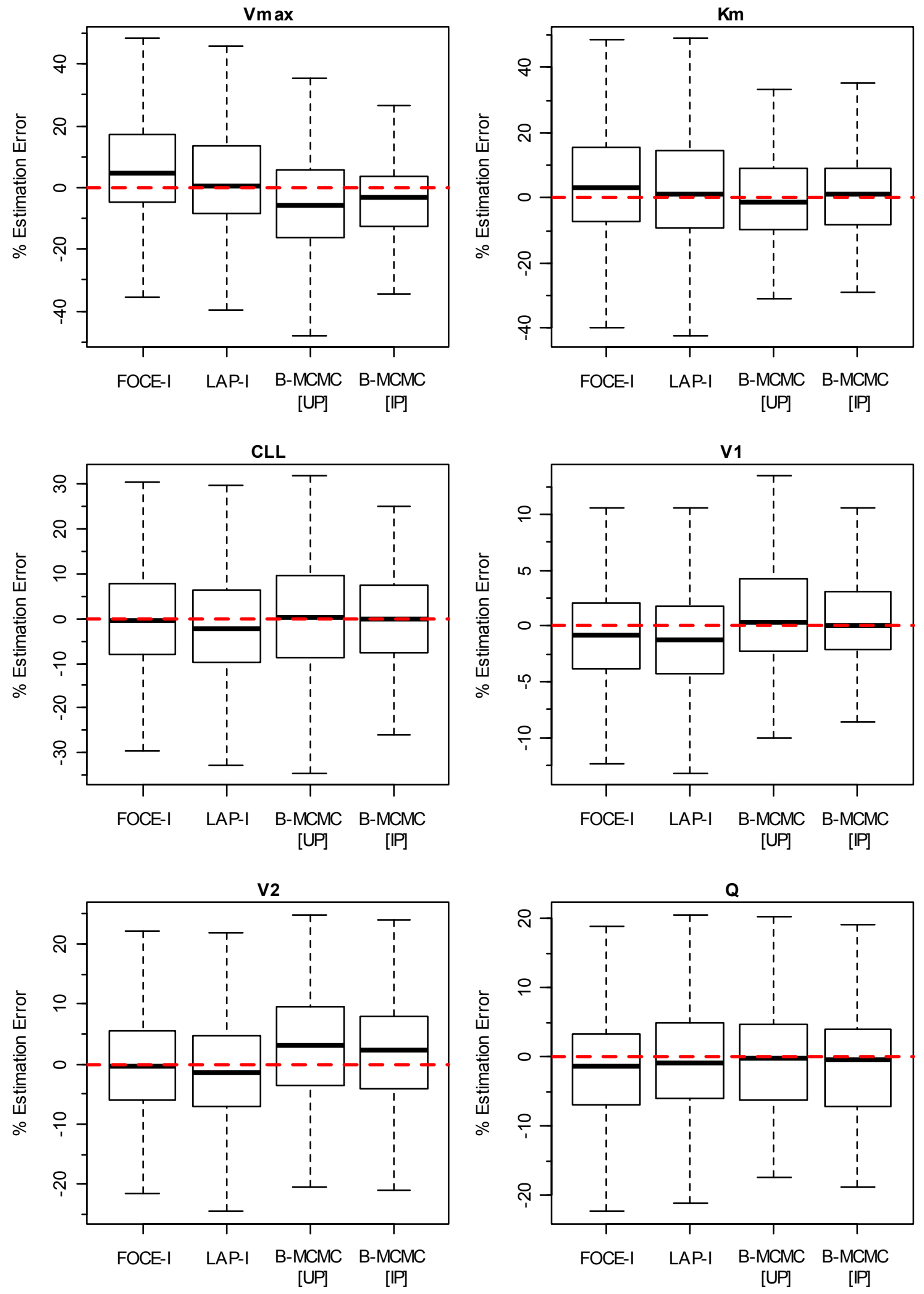

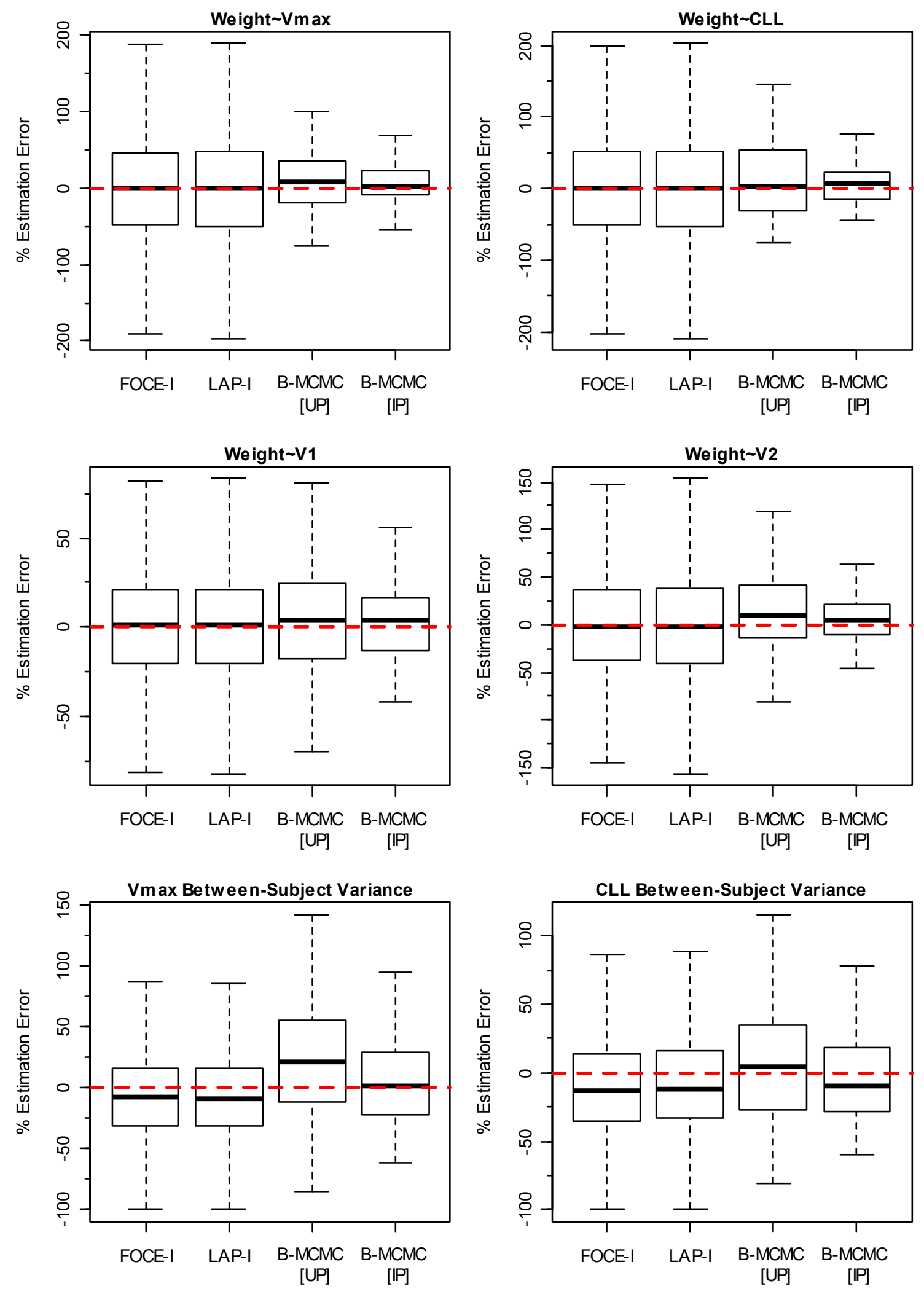

Figure 4-2 continued. 

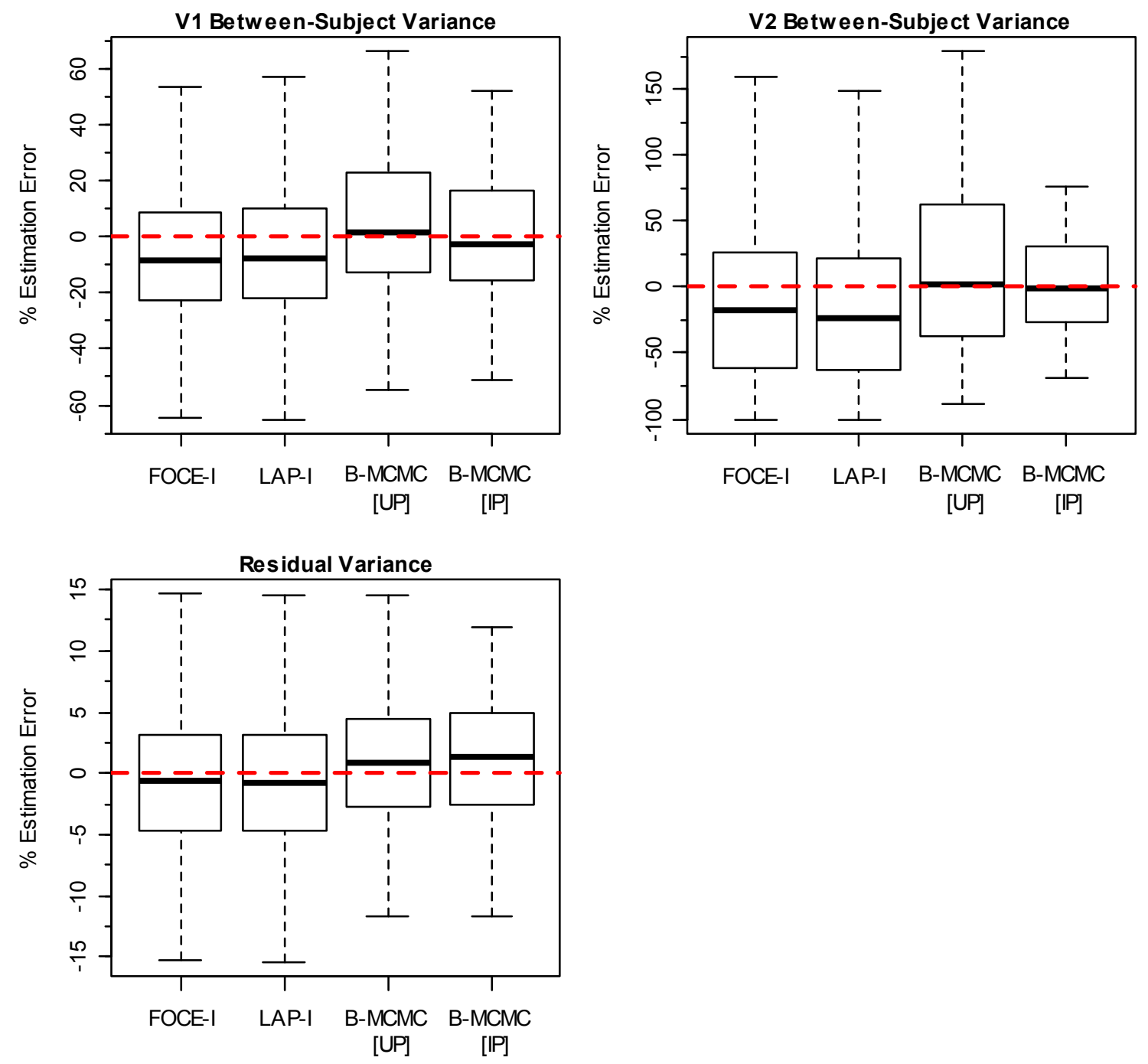

Figure 4-2 continued. 
Select plots from the global sensitivity analysis under the informative study design are shown in Figure 4-3 for the covariate effects and between-subject variances. Similar results were observed in the global sensitivity analysis for FOCE-I and LAP-I, therefore plots for FOCE-I are only shown. For each estimation method, with the exception of when informative Bayesian priors were used, the sensitivity plots showed that for all parameters the percent estimation errors were insensitive to uncertainty in the values of the simulation parameters. In other words, no trends were observed in the parameter estimation errors when viewed across the range of parameter uncertainty employed for simulating the replicate data sets. In the case of informative priors, the sensitivity plots for the weight effect and the between-subject variance for $\mathrm{V}_{\max }, \mathrm{CL}_{\mathrm{L}}$, and $\mathrm{V}_{2}$ showed an increase in bias as simulation parameter values were selected further away from the mean or midpoint of their respective informative prior distribution. For reference, the geometric means of the informative priors for the between-subject variability in $\mathrm{V}_{\max }, \mathrm{CL}_{\mathrm{L}}$, and $\mathrm{V}_{2}$ in terms of variance were $0.1225,0.1225$, and 0.0625 , respectively. The midpoints of the informative priors for the covariate weight effect were each 0.75 .

Predictive checks showed that the parameter biases observed under the informative study design for FOCE-I, LAP-I, and Bayesian MCMC with informative and uninformative priors would not result in predictions that differed significantly from the true model. This was expected given the relatively small parameter biases seen in Table 4-1. For the true and biased parameter values, 1000 data sets were simulated using an informative study design template data set, and the simulated concentrations during the full concentration-time profile at week 4 of therapy were then compared. As seen in Figures 4-4 and 4-5 for FOCE-I and Bayesian MCMC with uninformative priors, the analysis-derived median and $90 \%$ prediction interval for concentrations during week 4 were almost identical to the true model predicitons across all dose levels in the informative study design. Similar plots were observed for LAP-I and Bayesian MCMC with informative priors (not shown).

\section{Uninformative $600 \mathrm{mg}$ Dose Level Study Design}

Bias and precision of the population PK model parameters under the uninformative $600 \mathrm{mg}$ dose level study design are summarized in Tables 4-3 and 4-4, and boxplots of percent estimation errors for the model parameters are shown in Figure 4-6. The bias for most parameters under FOCE-I and LAP-I was less than $\pm 20 \%$. Parameter biases were comparable between FOCE-I and LAP-I, although LAP-I produced a noticeably smaller bias for $\mathrm{V}_{\max }$ and the between-subject variance for $\mathrm{V}_{\max }$ and $\mathrm{V}_{2}$ compared to FOCE-I. The largest parameter biases (MPE) observed under FOCE-I were for $\mathrm{V}_{\max }(42 \%), \mathrm{K}_{\mathrm{m}}(129 \%), \mathrm{V}_{\max }$ between-subject variance $(-40 \%)$, and $\mathrm{V}_{2}$ betweensubject variance $(-100 \%)$, while the largest biases observed under LAP-I were for $\mathrm{K}_{\mathrm{m}}$ $(143 \%)$ and $\mathrm{V}_{2}$ between-subject variance $(-53 \%)$. When informative Bayesian priors were used, the bias for all parameters was less than $\pm 10 \%$, with the only exception being $\mathrm{K}_{\mathrm{m}}$ which had an MPE of 34\%. The use of informative priors produced smaller biases for most of the model parameters compared to FOCE-I and/or LAP-I, and this was 
Figure 4-3. Global sensitivity analysis results from the informative study design. Plots are shown for selected parameters where the percent estimation error is plotted relative to the specific value of the simulation model parameter for each replicate. The red line indicates a LOESS smoothing curve. Panels A-D show sensitivity plots for the between-subject variances for $V_{\max }, C_{L}, V_{1}$, and $V_{2}$, respectively. Panels E-H show sensitivity plots for the weight effect on $\mathrm{V}_{\max }, \mathrm{CL}_{\mathrm{L}}, \mathrm{V}_{1}$, and $\mathrm{V}_{2}$, respectively. Note that the $\mathrm{y}$-axis scale differs from one plot to another. $\mathbf{B S V}=$ between-subject variance; $\mathbf{I P}=$ informative priors; $\mathbf{\%} \mathbf{P E}=$ percent estimation error; $\mathbf{U P}=$ uninformative priors. 

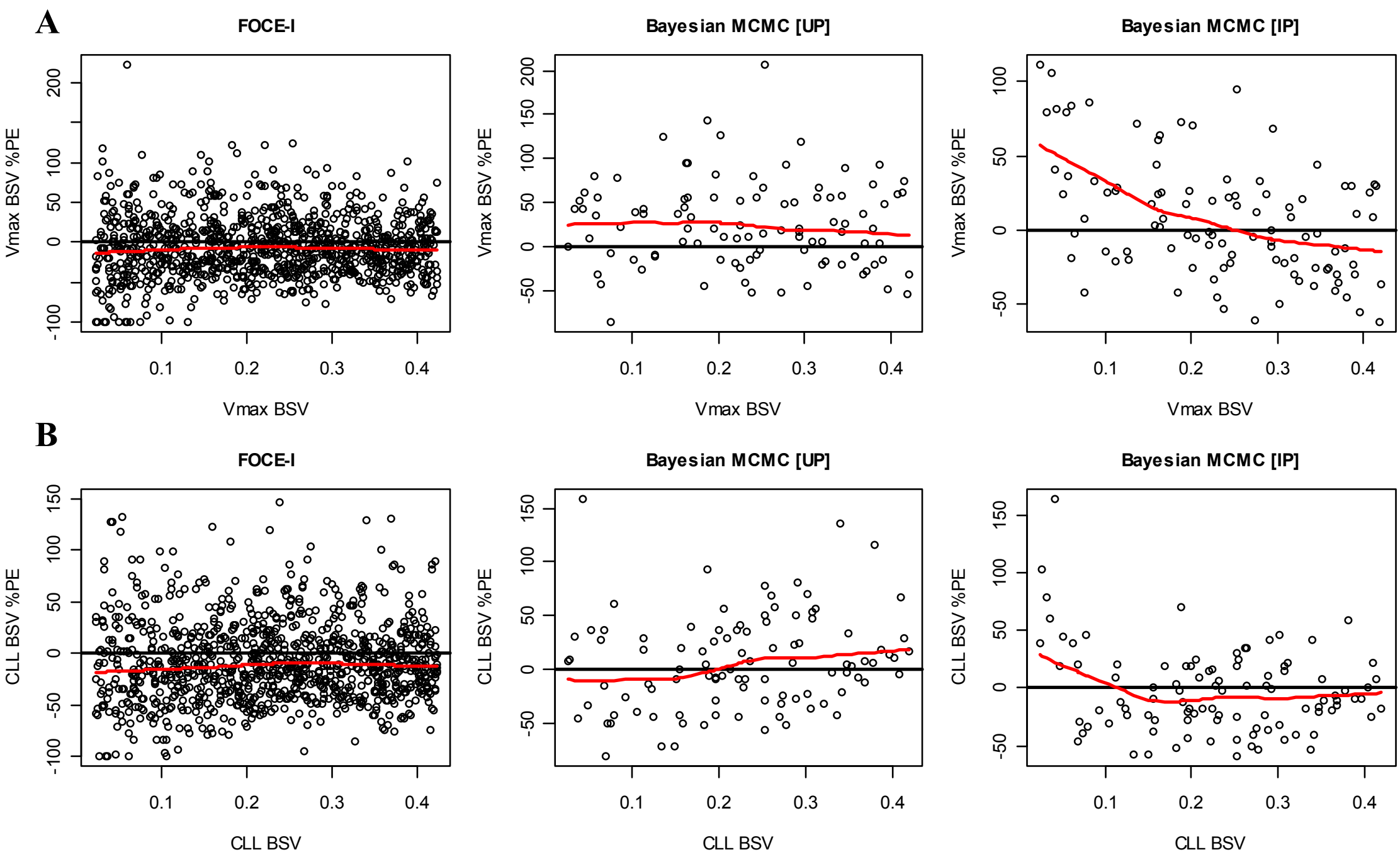


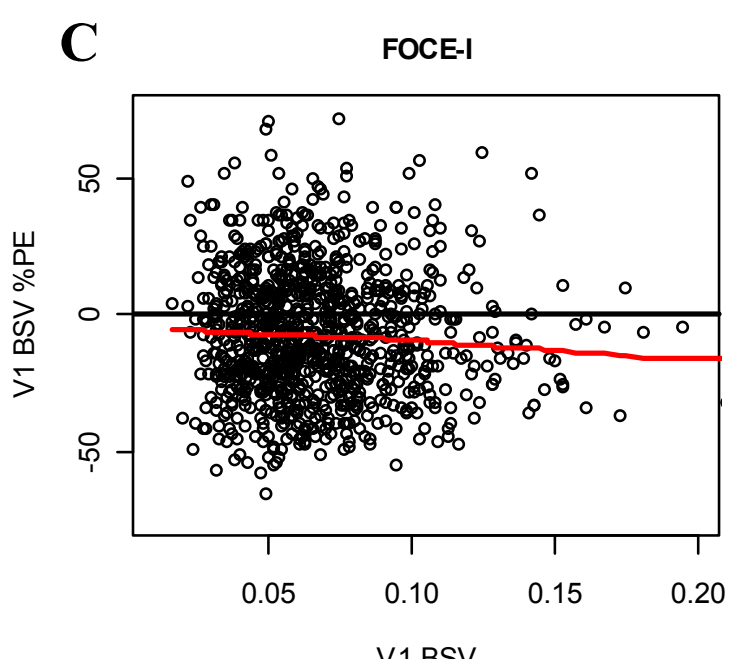

D

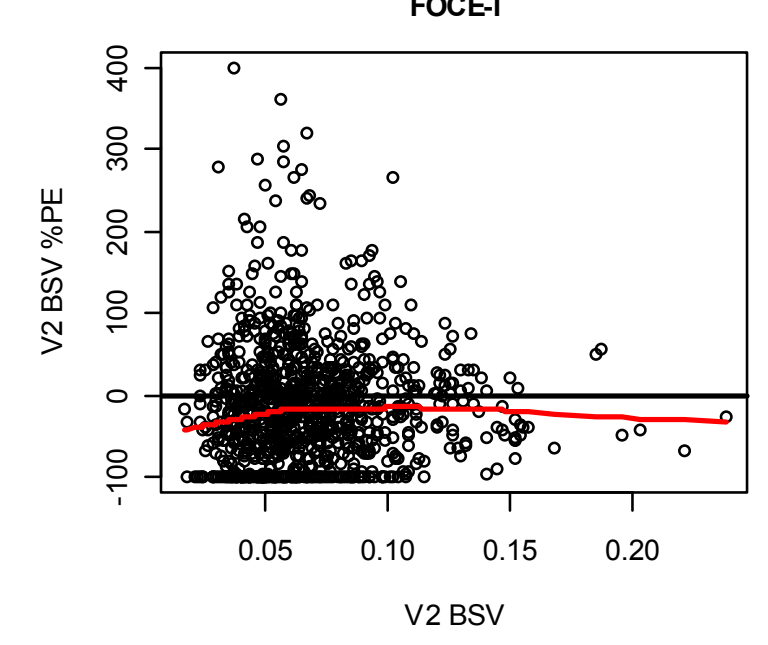

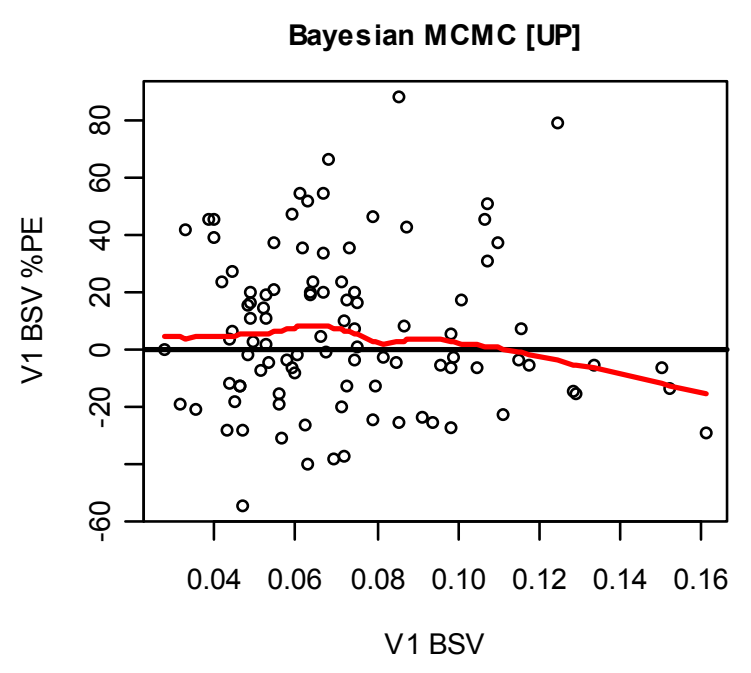

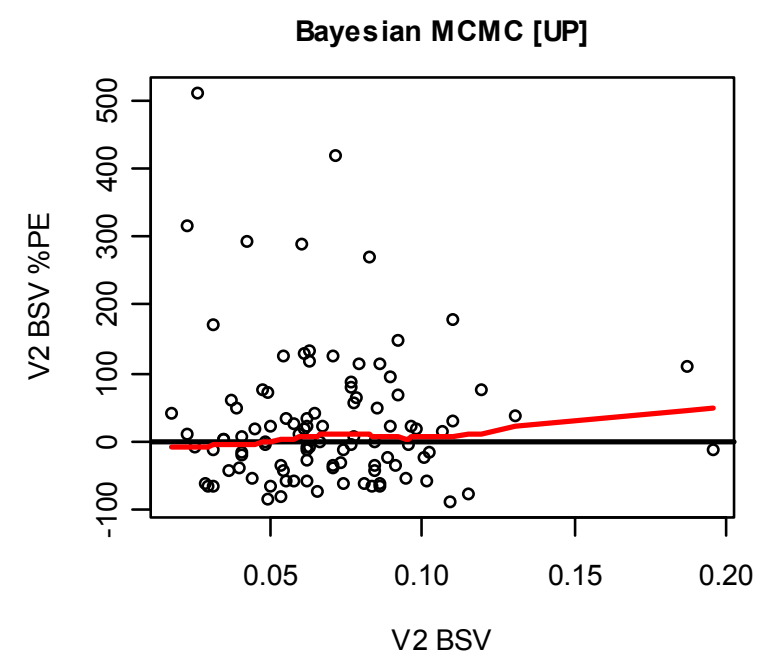

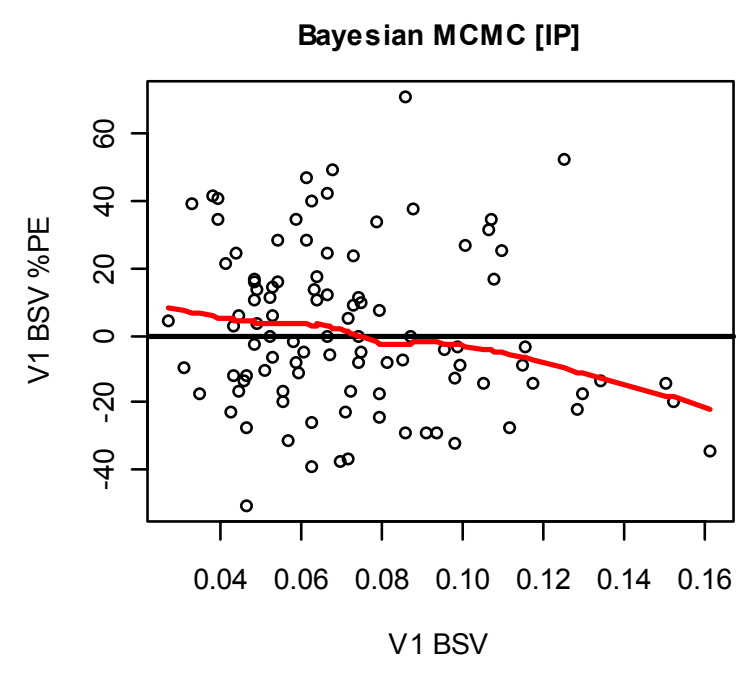

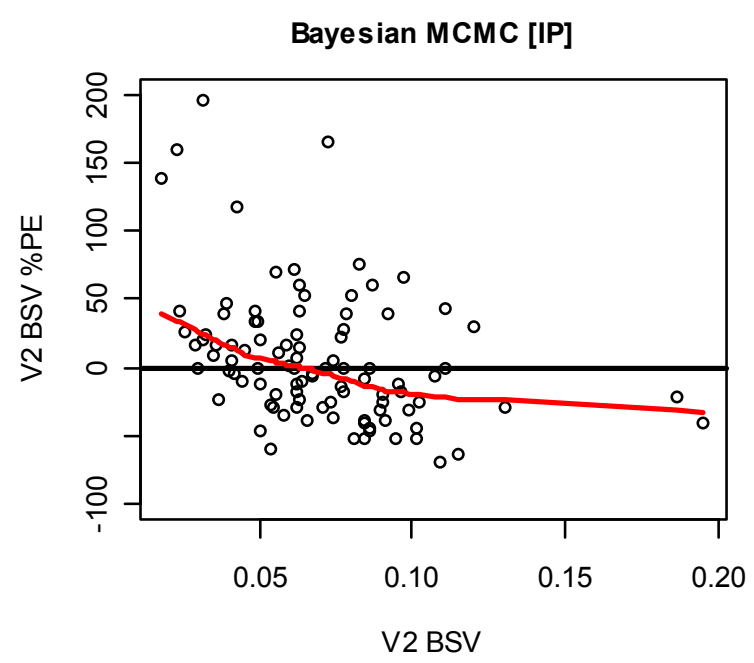

Figure 4-3 continued. 

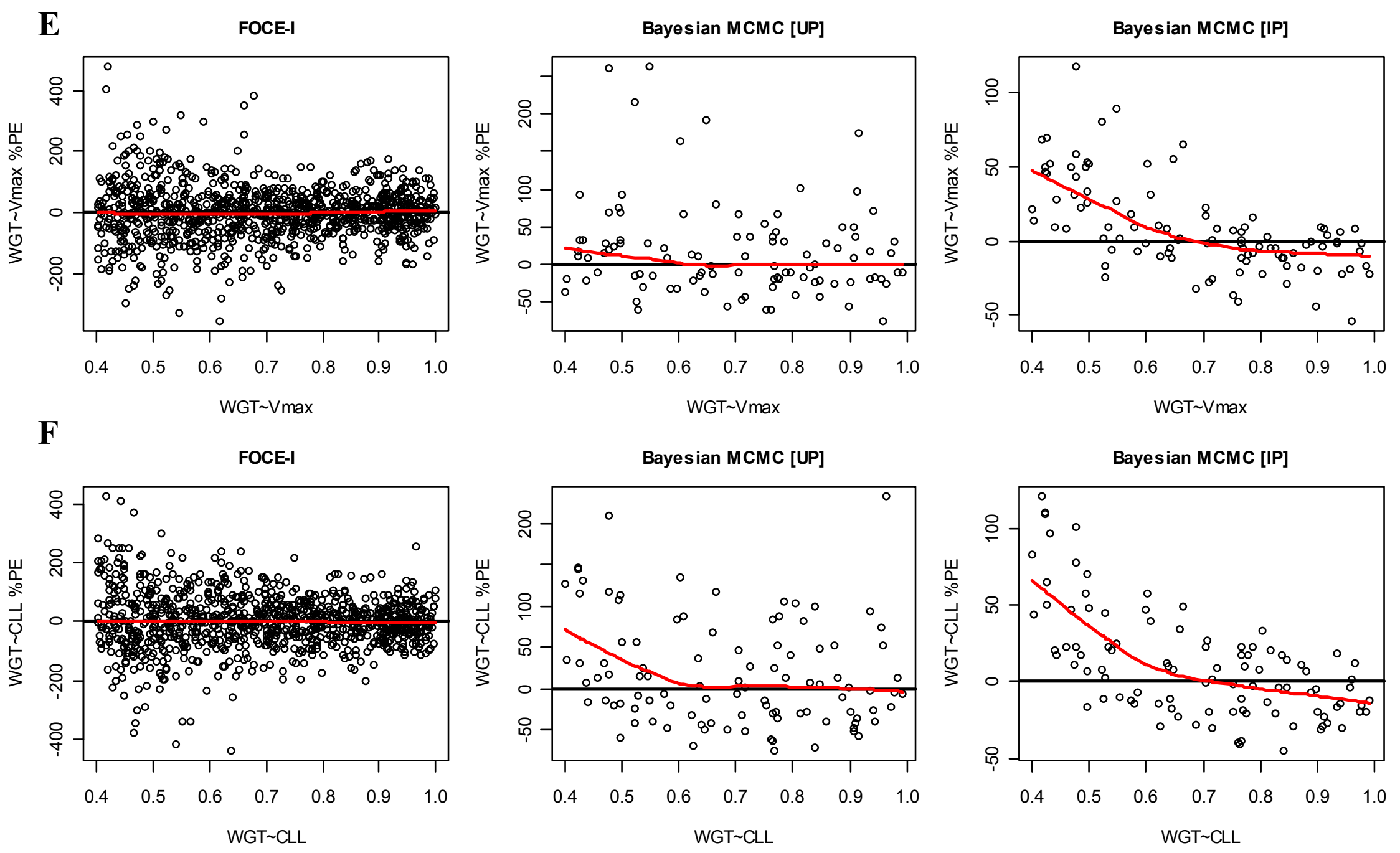

Figure 4-3 continued. 

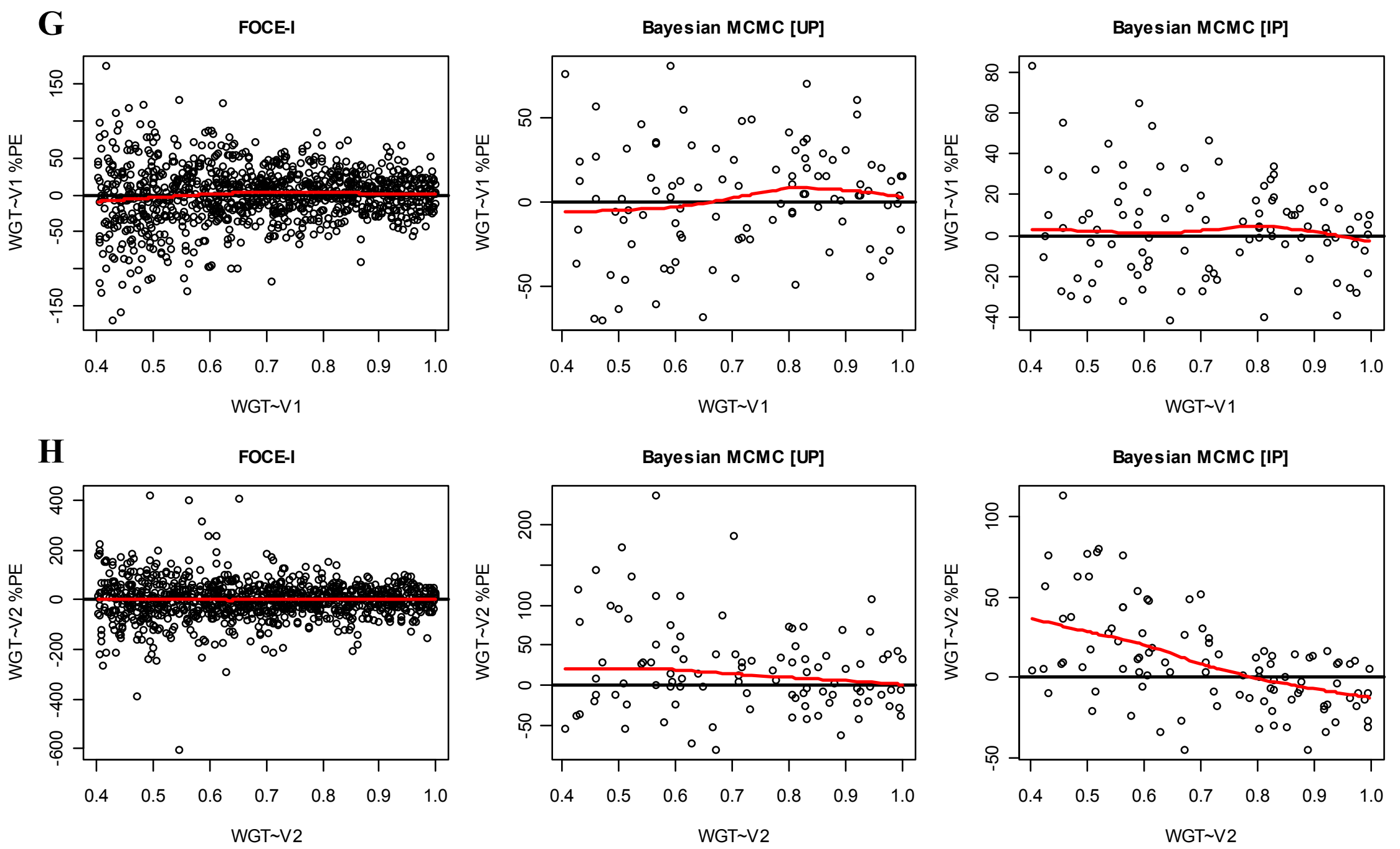

Figure 4-3 continued. 
$50 \mathrm{mg}$

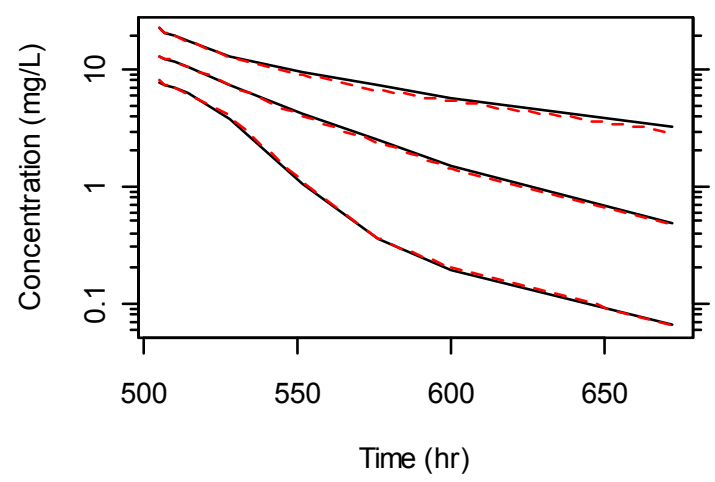

$200 \mathrm{mg}$

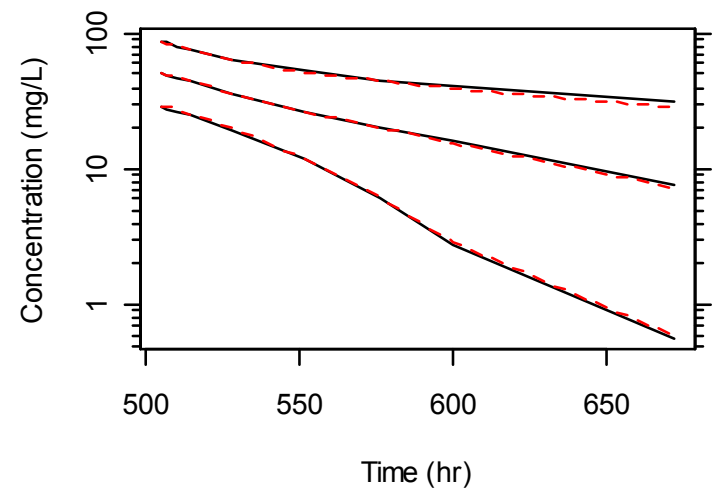

$800 \mathrm{mg}$

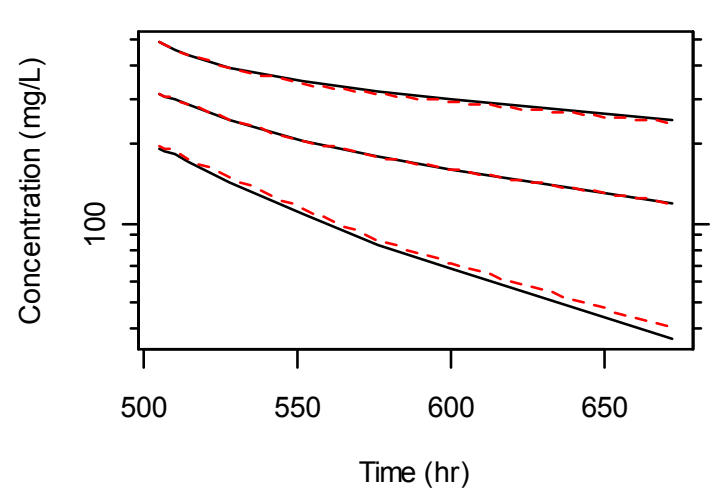

$100 \mathrm{mg}$

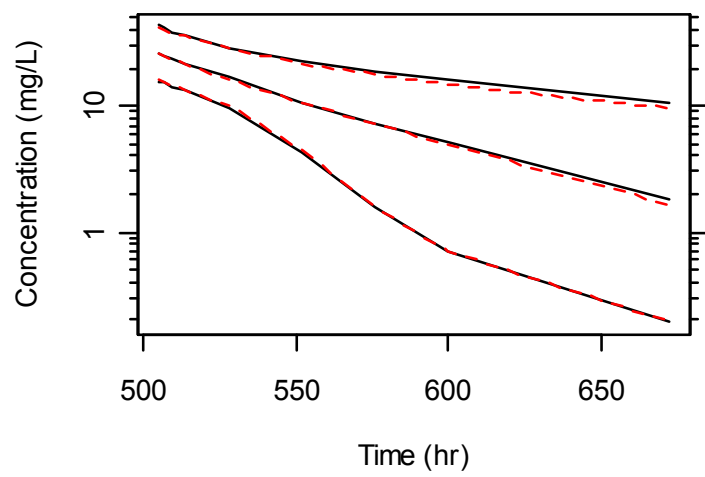

$400 \mathrm{mg}$

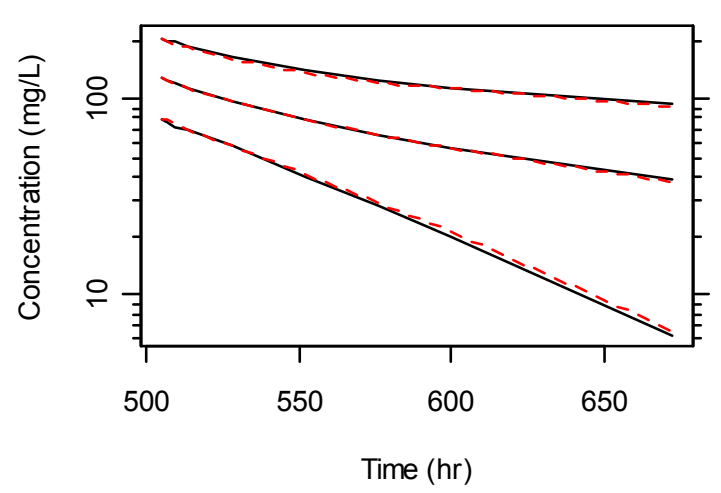

$1600 \mathrm{mg}$

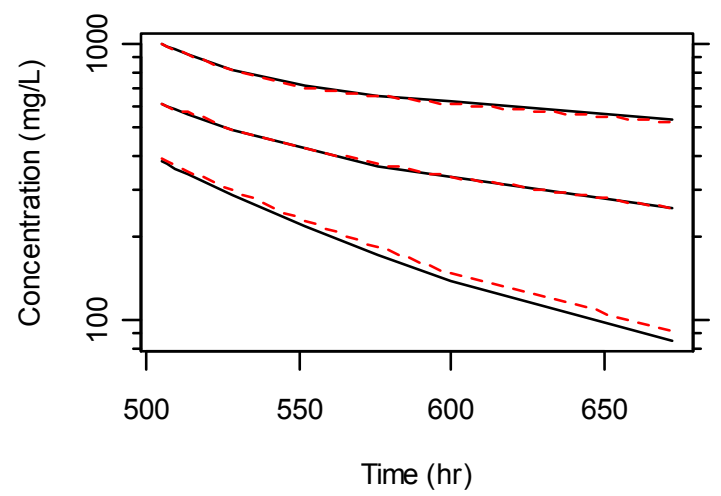

Figure 4-4. Simulated prediction intervals for concentrations during week 4 of therapy based on the true parameter values and biased parameter values derived from the FOCE-I results under the informative study design. The true model and analysis-derived predictions are indicated by the solid black lines and dashed red lines, respectively. The three lines represent the 5th, 50th, and 95th percentiles determined from 1000 simulated data sets. The simulations were carried out using an informative study design template data set. Simulated prediction intervals are shown separately for each dose group. 
$50 \mathrm{mg}$

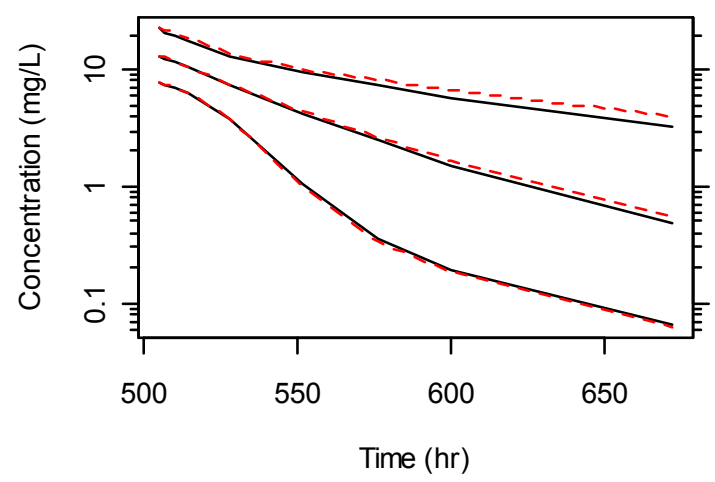

$200 \mathrm{mg}$

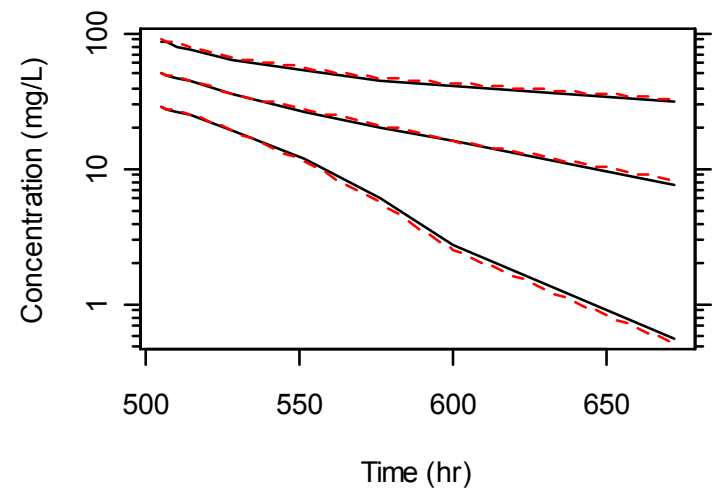

$800 \mathrm{mg}$

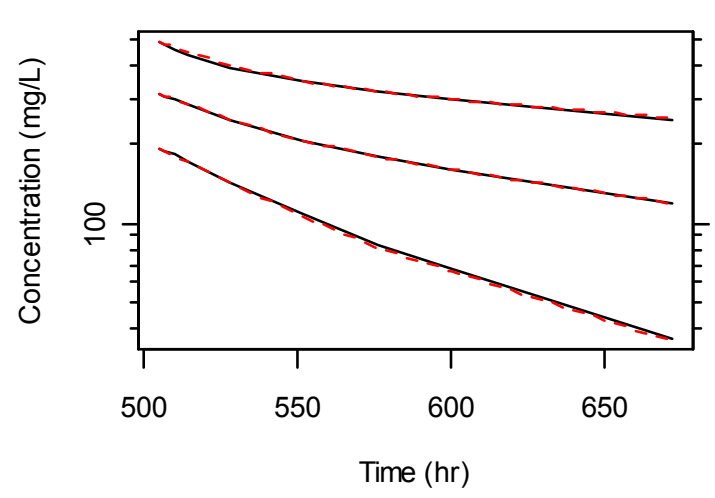

$100 \mathrm{mg}$

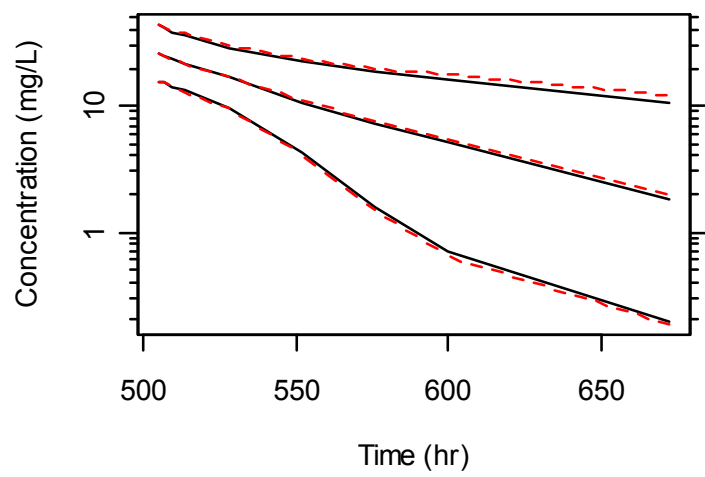

$400 \mathrm{mg}$

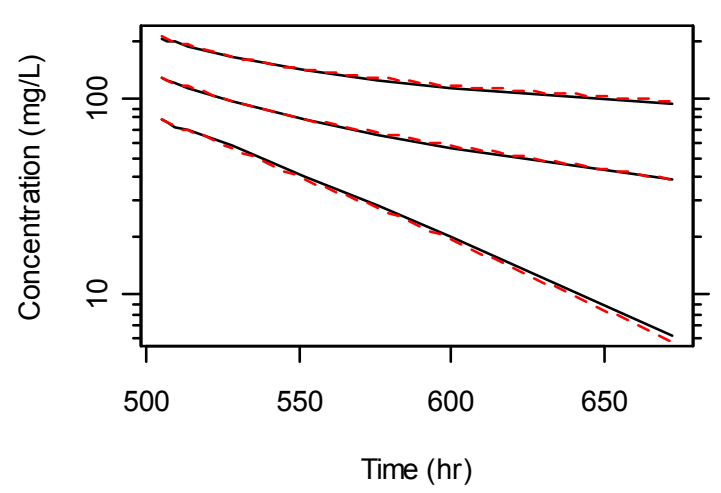

$1600 \mathrm{mg}$

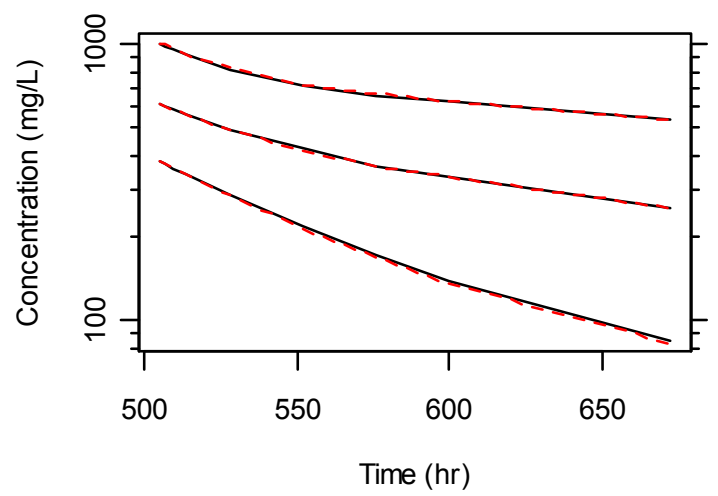

Figure 4-5. Simulated prediction intervals for concentrations during week 4 of therapy based on the true parameter values and biased parameter values derived from the Bayesian MCMC (with uninformative priors) results under the informative study design. The true model and analysis-derived predictions are indicated by the solid black lines and dashed red lines, respectively. The three lines represent the 5th, 50th, and 95th percentiles determined from 1000 simulated data sets. The simulations were carried out using an informative study design template data set. Simulated prediction intervals are shown separately for each dose group. 
Table 4-3. Bias (median percent estimation error) in the population pharmacokinetic model parameters under the uninformative $600 \mathrm{mg}$ dose level study design.

\begin{tabular}{|c|c|c|c|c|}
\hline Parameter & FO & FOCE-I & LAP-I & $\begin{array}{l}\text { B-MCMC } \\
\text { [IP] }\end{array}$ \\
\hline $\mathrm{V}_{\max }$ & 75 & 42 & 12 & 7.4 \\
\hline $\mathrm{K}_{\mathrm{m}}$ & 446 & 129 & 173 & 34 \\
\hline $\mathrm{CL}_{\mathrm{L}}$ & -21 & -12 & -6.5 & -5.9 \\
\hline $\mathrm{V}_{1}$ & -4.0 & -1.4 & -1.3 & -0.43 \\
\hline $\mathrm{V}_{2}$ & 23 & 0.23 & -2.0 & 4.7 \\
\hline Q & 20 & 3.4 & 2.2 & 2.6 \\
\hline $\mathrm{WGT} \sim \mathrm{V}_{\max }$ & -18 & 3.8 & 5.2 & 9.6 \\
\hline $\mathrm{WGT} \sim \mathrm{CL}_{\mathrm{L}}$ & -17 & -18 & -11 & 4.7 \\
\hline $\mathrm{WGT} \sim \mathrm{V}_{1}$ & -1.9 & -0.28 & -0.23 & 0.63 \\
\hline $\mathrm{WGT} \sim \mathrm{V}_{2}$ & 46 & 21 & 18 & 3.1 \\
\hline$V_{\max } \operatorname{BSV}\left(\omega^{2}\right)$ & -75 & -40 & 5.9 & -8.4 \\
\hline $\mathrm{CL}_{\mathrm{L}} \mathrm{BSV}\left(\omega^{2}\right)$ & -1.9 & -0.92 & -4.9 & 0.77 \\
\hline$V_{1} \operatorname{BSV}\left(\omega^{2}\right)$ & -6.0 & -7.1 & -5.9 & -8.7 \\
\hline$V_{2} \operatorname{BSV}\left(\omega^{2}\right)$ & 57 & -100 & -53 & -2.4 \\
\hline Residual variance $\left(\sigma^{2}\right)$ & 6.9 & -2.6 & -2.3 & 0.40 \\
\hline
\end{tabular}

B-MCMC = Bayesian Markov Chain Monte Carlo; $\mathbf{B S V}=$ between-subject variance; $\mathbf{I P}=$ informative priors; $\mathbf{W G T}=$ weight. 
Table 4-4. Precision (median absolute percent estimation error) in the population pharmacokinetic model parameters under the uninformative $600 \mathrm{mg}$ dose level study design.

\begin{tabular}{|c|c|c|c|c|}
\hline Parameter & FO & FOCE-I & LAP-I & $\begin{array}{l}\text { B-MCMC } \\
{[\mathrm{IP}]}\end{array}$ \\
\hline $\mathrm{V}_{\max }$ & 100 & 54 & 53 & 25 \\
\hline $\mathrm{K}_{\mathrm{m}}$ & 446 & 129 & 173 & 38 \\
\hline $\mathrm{CL}_{\mathrm{L}}$ & 26 & 17 & 16 & 9.5 \\
\hline $\mathrm{V}_{1}$ & 4.5 & 3.3 & 3.3 & 2.9 \\
\hline $\mathrm{V}_{2}$ & 29 & 16 & 17 & 9.8 \\
\hline Q & 24 & 16 & 17 & 12 \\
\hline $\mathrm{WGT} \sim \mathrm{V}_{\max }$ & 119 & 99 & 97 & 18 \\
\hline $\mathrm{WGT} \sim \mathrm{CL}_{\mathrm{L}}$ & 101 & 83 & 73 & 16 \\
\hline $\mathrm{WGT} \sim \mathrm{V}_{1}$ & 27 & 23 & 24 & 17 \\
\hline $\mathrm{WGT} \sim \mathrm{V}_{2}$ & 120 & 102 & 112 & 19 \\
\hline $\mathrm{V}_{\max } \operatorname{BSV}\left(\omega^{2}\right)$ & 100 & 81 & 79 & 43 \\
\hline $\mathrm{CL}_{\mathrm{L}} \mathrm{BSV}\left(\omega^{2}\right)$ & 61 & 32 & 28 & 17 \\
\hline $\mathrm{V}_{1} \operatorname{BSV}\left(\omega^{2}\right)$ & 22 & 20 & 19 & 19 \\
\hline $\mathrm{V}_{2} \operatorname{BSV}\left(\omega^{2}\right)$ & 100 & 100 & 100 & 30 \\
\hline Residual variance $\left(\sigma^{2}\right)$ & 8.3 & 5.2 & 5.7 & 4.6 \\
\hline
\end{tabular}

B-MCMC = Bayesian Markov Chain Monte Carlo; $\mathbf{B S V}=$ between-subject variance; $\mathbf{I P}=$ informative priors; $\mathbf{W G T}=$ weight. 
Figure 4-6. Boxplots of percent estimation errors for the population pharmacokinetic model parameters under the uninformative $600 \mathrm{mg}$ dose level study design. Outliers are not shown for visualization purposes and made up $<10 \%$ of the data for each parameterestimation method combination. The median percent estimation error corresponds to the calculated parameter bias. Note that the y-axis scale differs from one boxplot to another. B-MCMC = Bayesian Markov Chain Monte Carlo; IP = informative priors. 

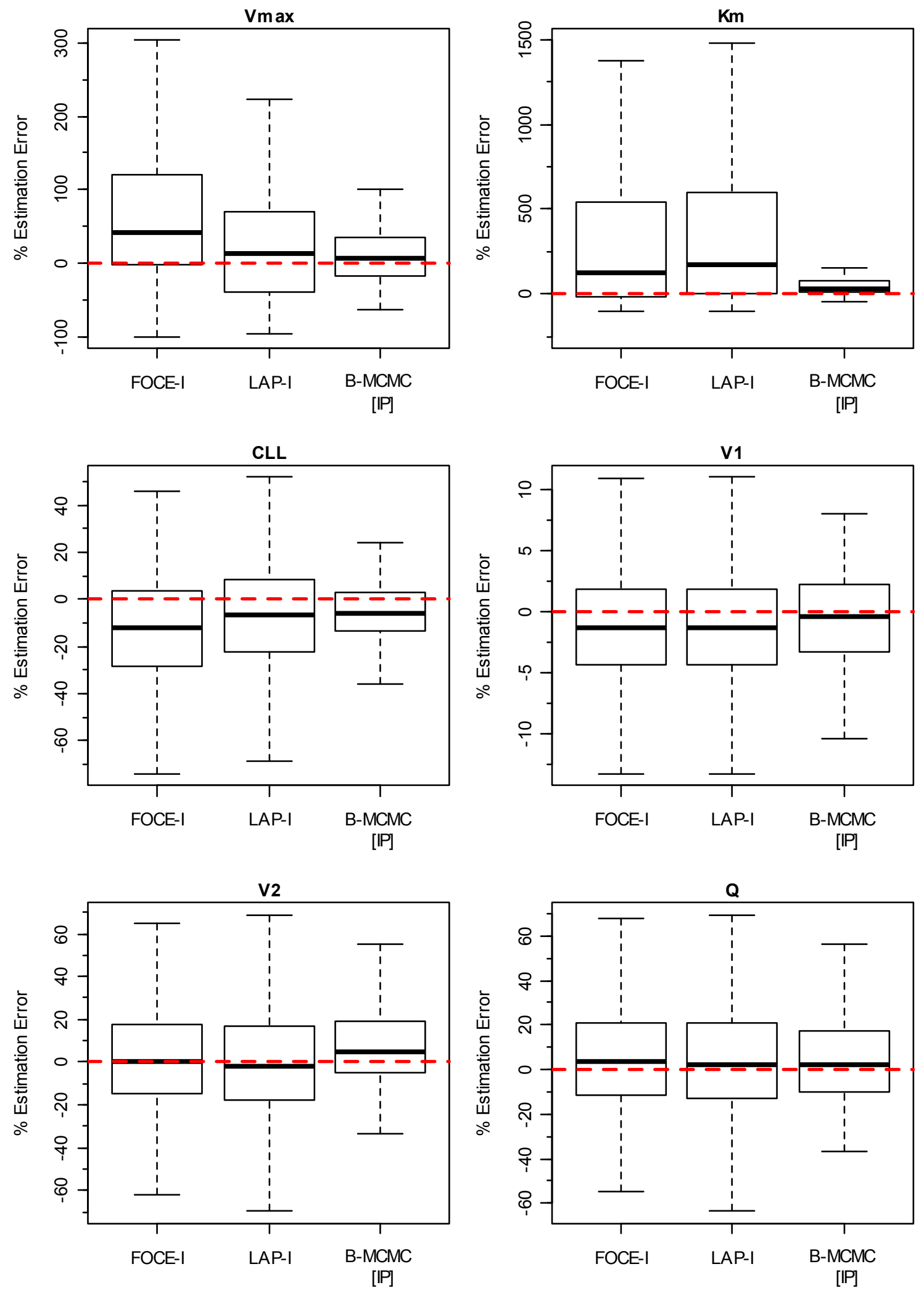

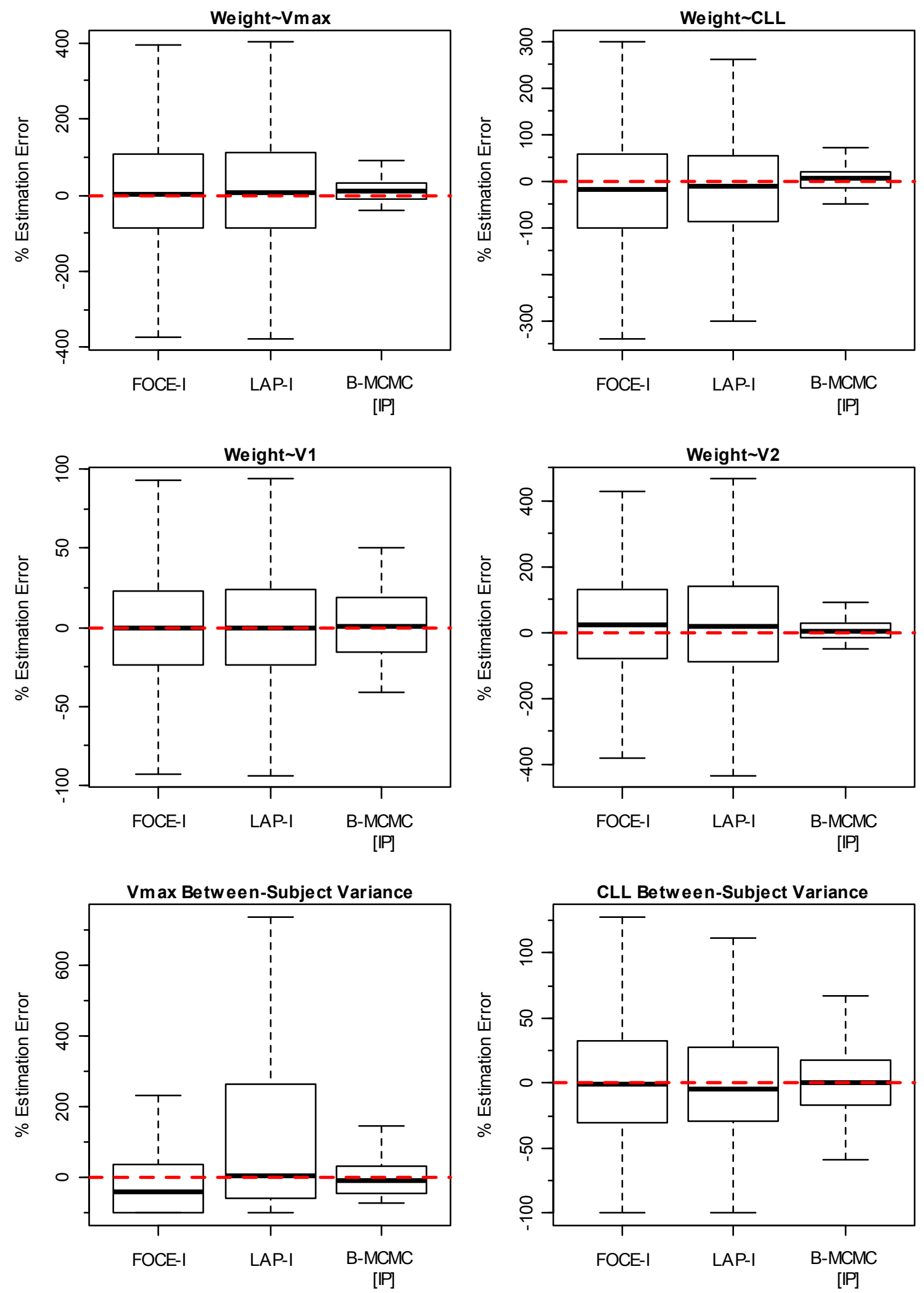

Figure 4-6 continued. 

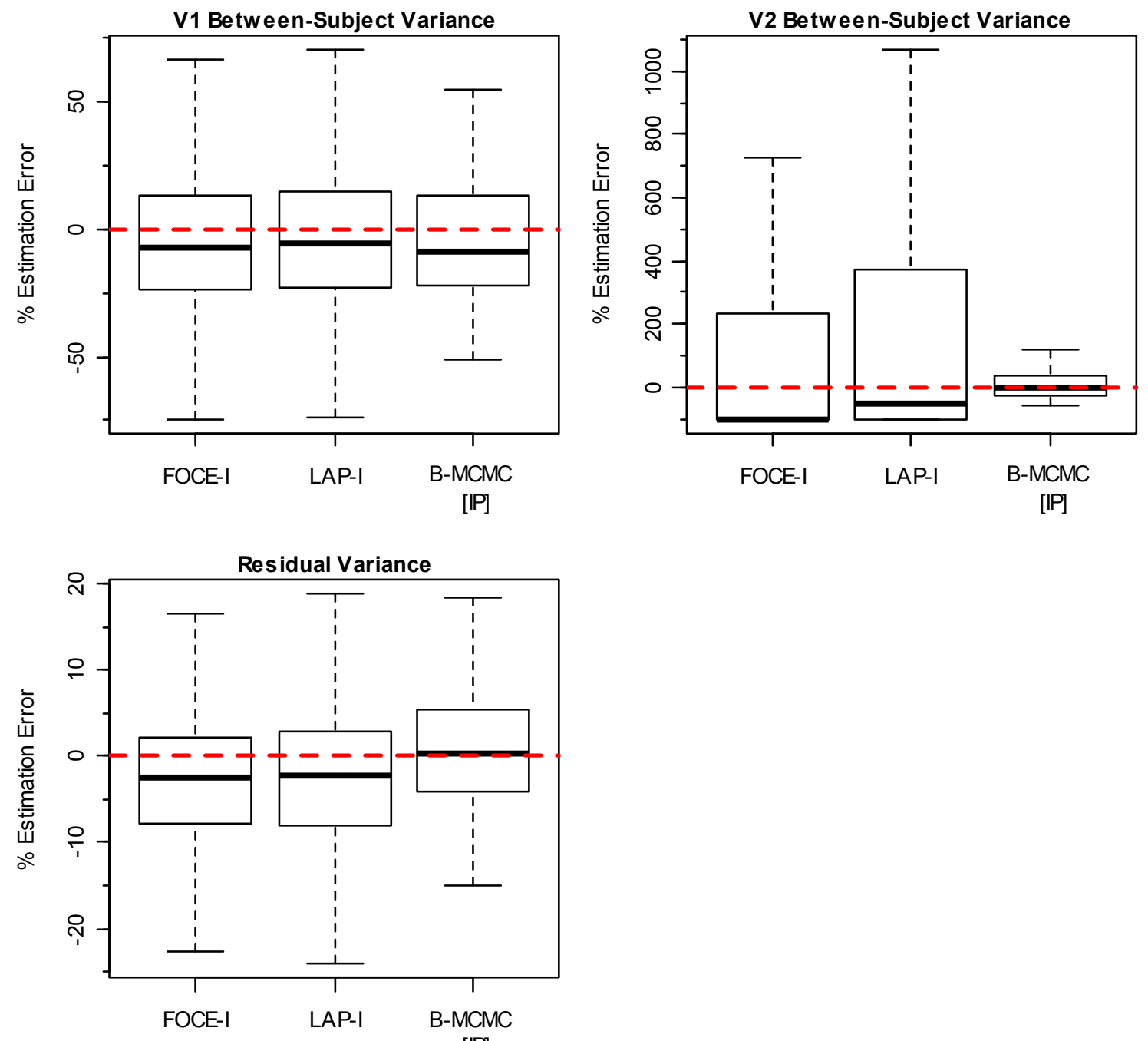

Figure 4-6 continued. 
particularly evident for $\mathrm{V}_{\max }, \mathrm{K}_{\mathrm{m}}$, the weight effect on $\mathrm{CL}_{\mathrm{L}}$ and $\mathrm{V}_{2}$, and the betweensubject variance for $V_{\max }$ and $V_{2}$ (Table 4-3 and Figure 4-6).

FOCE-I and LAP-I produced similar results with regards to parameter precision, but both estimation methods exhibited low precision as almost half of the model parameters had a MAPE greater than $50 \%$. These parameters included $\mathrm{V}_{\max }, \mathrm{K}_{\mathrm{m}}$, the weight effect on $\mathrm{V}_{\max }, \mathrm{CL}_{\mathrm{L}}, \mathrm{V}_{2}$, and the between-subject variance for $\mathrm{V}_{\max }$ and $\mathrm{V}_{2}$. When informative Bayesian priors were used, the MAPE was less than 30\% for all parameters with the exception of $\mathrm{K}_{\mathrm{m}}(38 \%)$ and $\mathrm{V}_{\max }$ between-subject variance (43\%). Compared to FOCE-I and LAP-I, the use of informative priors resulted in greater parameter precision and smaller MAPEs for all model parameters. This was most apparent for $\mathrm{V}_{\max }, \mathrm{K}_{\mathrm{m}}$, the weight effect on $\mathrm{V}_{\max }, \mathrm{CL}_{\mathrm{L}}, \mathrm{V}_{2}$, and the between-subject variance for $\mathrm{V}_{\max }$ and $\mathrm{V}_{2}$ (Table 4-4). The differences in parameter precision between the estimation methods were also evident in the boxplots in Figure 4-6, as the variability in the percent estimation errors for the aforementioned parameters was dramatically smaller when informative priors were used compared to FOCE-I and LAP-I.

The results for the estimation methods were also compared between the informative and uninformative $600 \mathrm{mg}$ dose level study designs to evaluate the performance of the methods as the amount of information in the dataset decreased and the nature of the study design made it more difficult to detect and characterize the nonlinear elimination of the therapeutic $\mathrm{mAb}$. The estimation performance of the methods was adequate and comparable under the informative study design, considering that the bias and precision for all model parameters was less than $\pm 25 \%$ and $52 \%$, respectively. Under the uninformative $600 \mathrm{mg}$ dose level study design, the performance of FOCE-I and LAPI decreased as the bias and precision for many of the parameters significantly increased to $\pm 40-173 \%$ and $53-173 \%$, respectively, while Bayesian MCMC with informative priors produced results that were comparable to those under the informative study design.

Select global sensitivity analysis plots are shown in Figure 4-7 for the betweensubject variance and weight effect parameters. For FOCE-I, LAP-I, and Bayesian MCMC with informative priors, the sensitivity plots showed that for most parameters the percent estimation errors were insensitive to uncertainty in the values of the simulation parameters. However, for each estimation method, trends were observed in the sensitivity plots for certain parameters. For FOCE-I and LAP-I, an increasing trend towards underpredicting the $\mathrm{V}_{2}$ between-subject variance was observed as smaller simulation parameter values were selected for the $V_{2}$ between-subject variance. $A$ similar observation was made for the $\mathrm{V}_{\max }$ between-subject variance under FOCE-I, where an increasing trend towards underpredicting the variance was observed as smaller between-subject variances for $\mathrm{V}_{\max }$ were selected from the simulation uncertainty distribution. When informative Bayesian priors were used, the sensitivity plots for the weight effect and the between-subject variance for $\mathrm{V}_{\max }, \mathrm{CL}_{\mathrm{L}}$, and $\mathrm{V}_{2}$ showed an increase in bias as simulation parameter values were selected far away from the mean or midpoint of their respective informative prior distribution. Similar plots were observed for these parameters in the global sensitivity analysis under the informative study design when informative Bayesian priors were used (Figure 4-3). 
Figure 4-7. Global sensitivity analysis results from the uninformative $600 \mathrm{mg}$ dose level study design. Plots are shown for selected parameters where the percent estimation error is plotted relative to the specific value of the simulation model parameter for each replicate. The red line indicates a LOESS smoothing curve. Panels A-D show sensitivity plots for the between-subject variances for $\mathrm{V}_{\max }, \mathrm{CL}_{\mathrm{L}}, \mathrm{V}_{1}$, and $\mathrm{V}_{2}$, respectively. Panels E-H show sensitivity plots for the weight effect on $\mathrm{V}_{\max }, \mathrm{CL}_{\mathrm{L}}, \mathrm{V}_{1}$, and $\mathrm{V}_{2}$, respectively. Note that the $y$-axis scale differs from one plot to another. $\mathbf{B S V}=$ between-subject variance; $\mathbf{I P}=$ informative priors; $\% \mathbf{P E}=$ percent estimation error. 
A

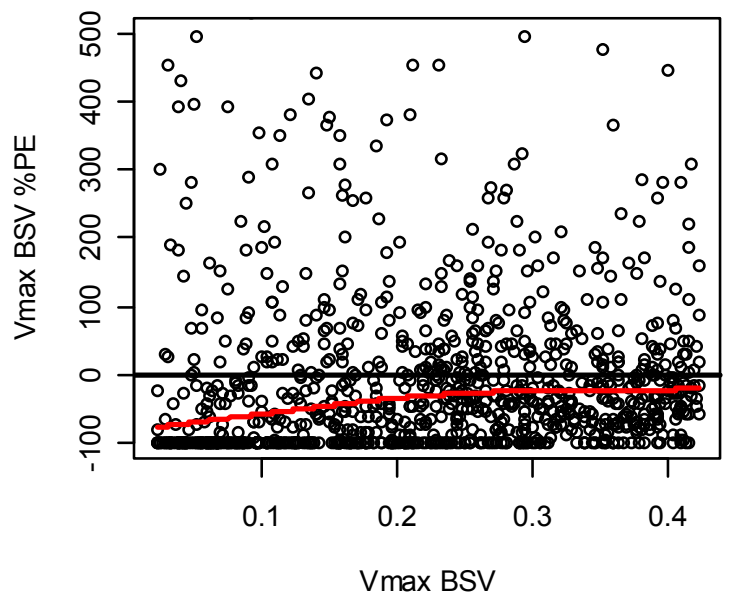

B

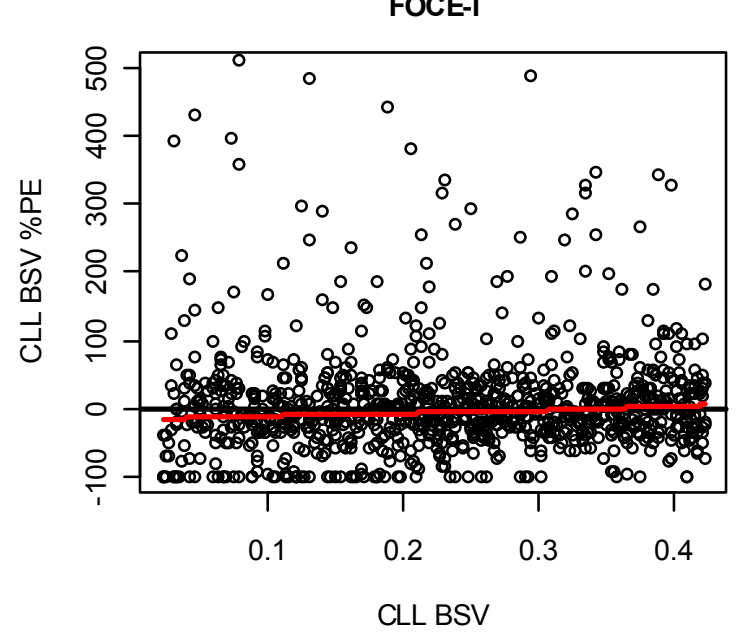

LAP-I

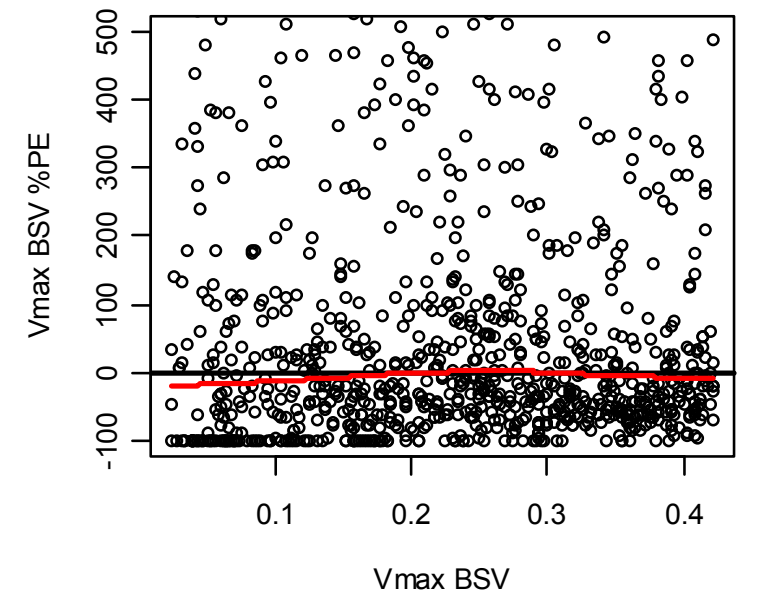

LAP-I

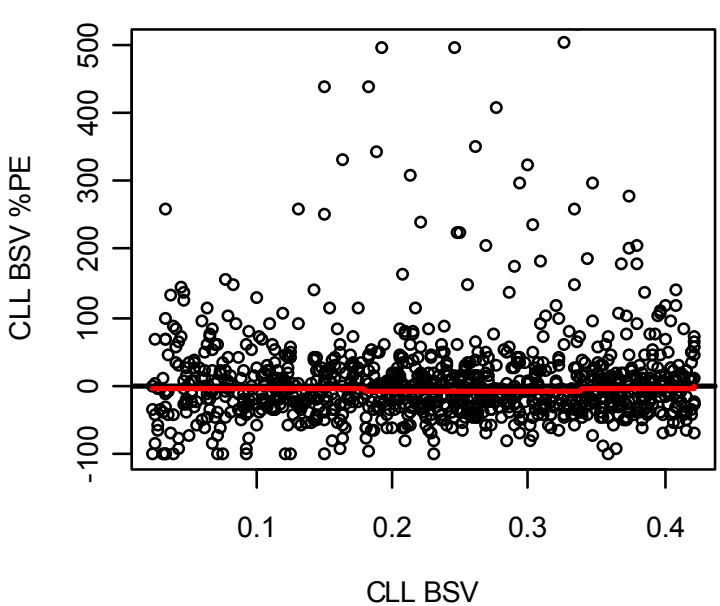

Bayesian MCMC [IP]

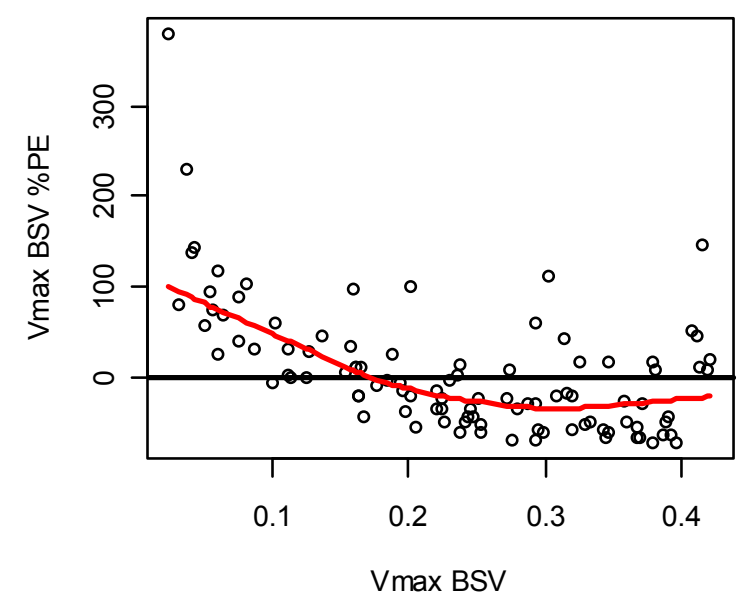

Bayesian MCMC [IP]

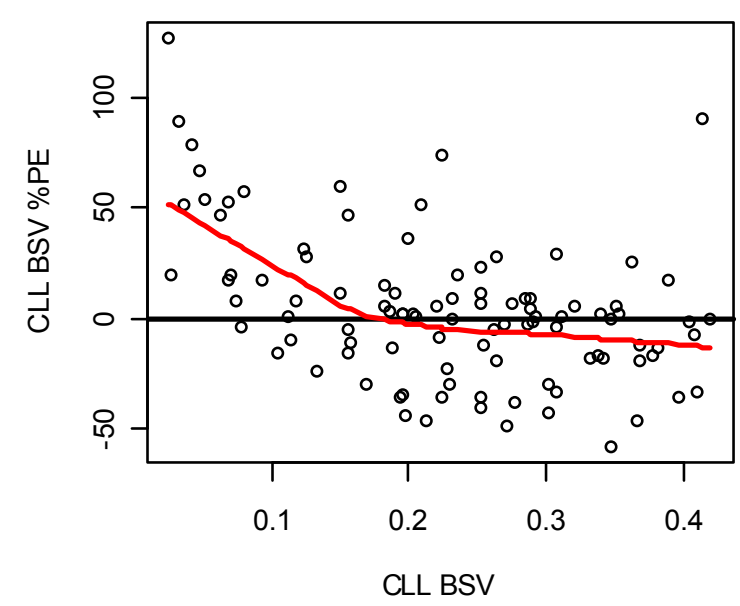




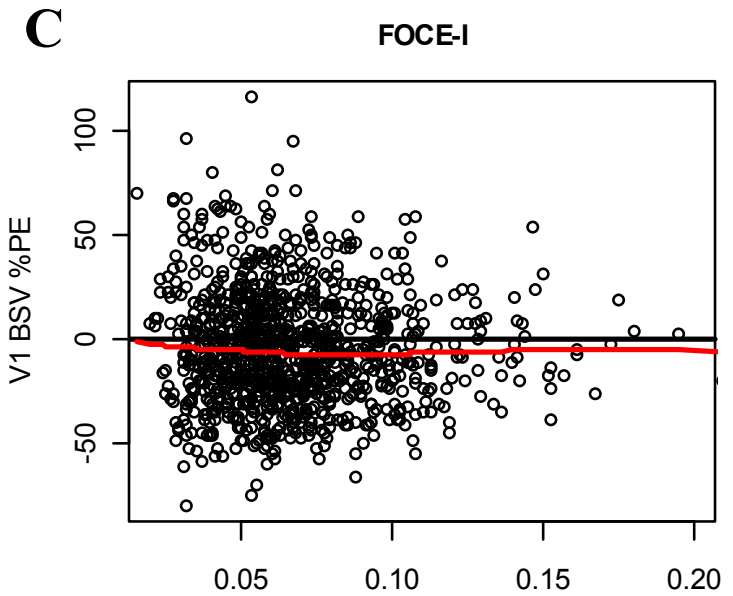

D

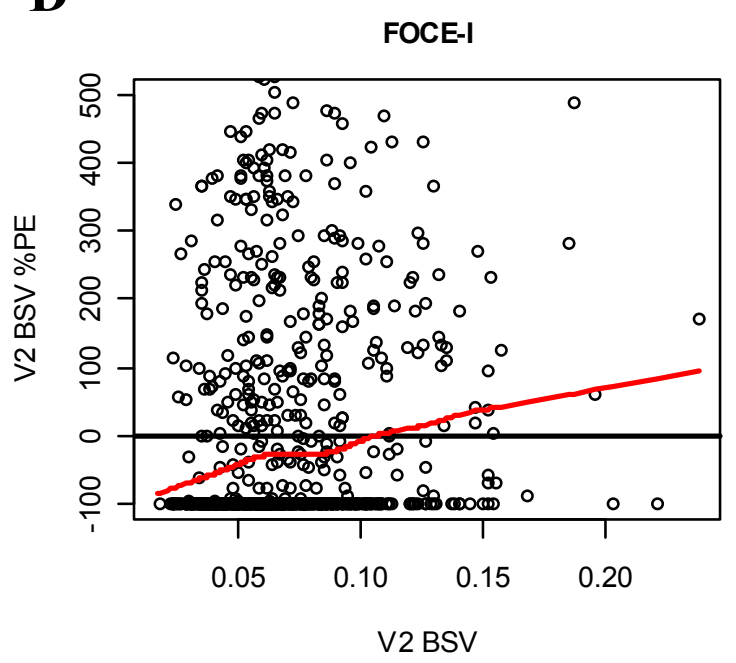

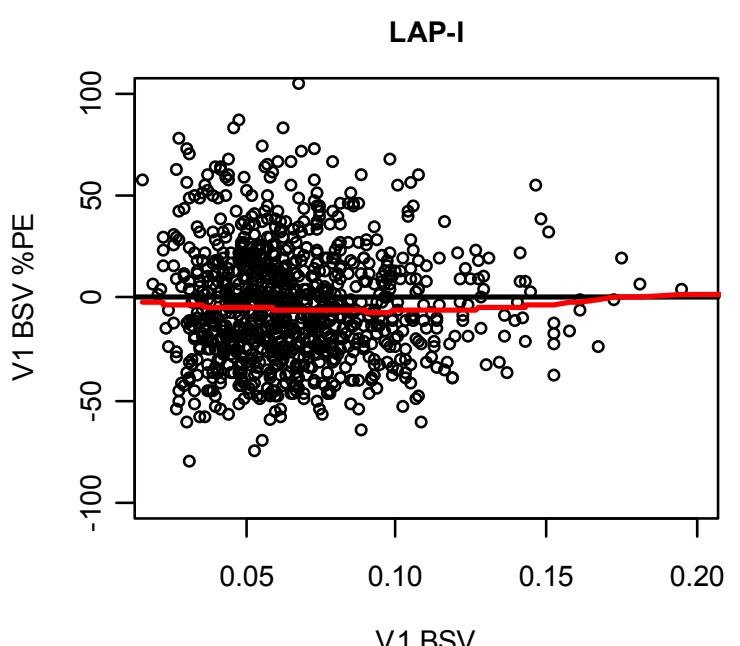

V1 BSV

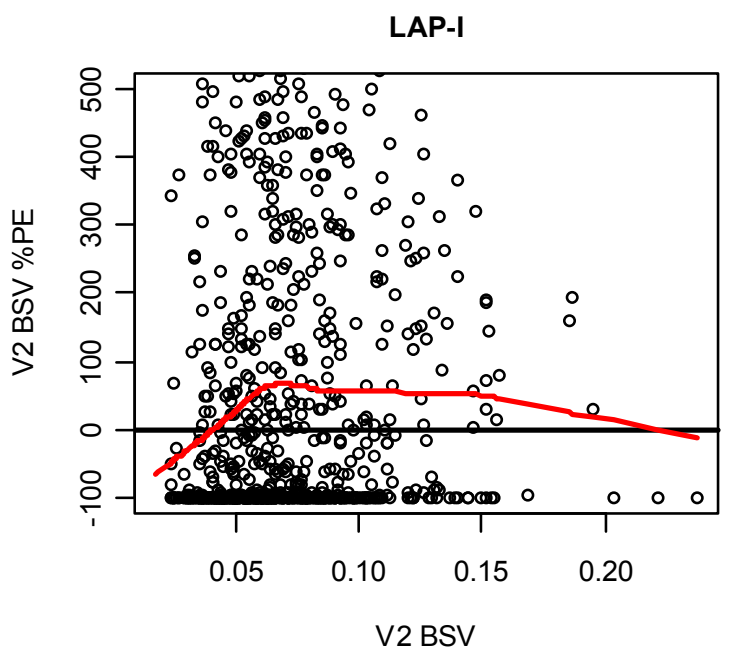

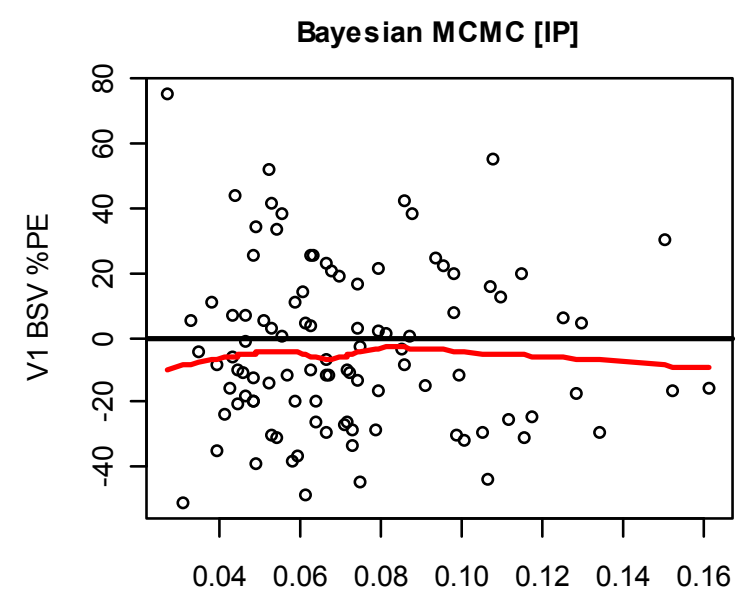

V1 BSV

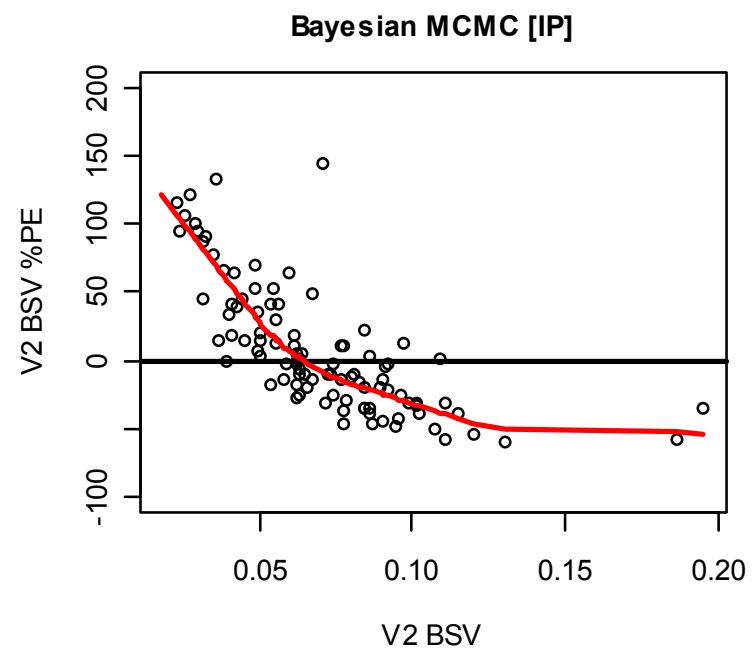

Figure 4-7 continued. 

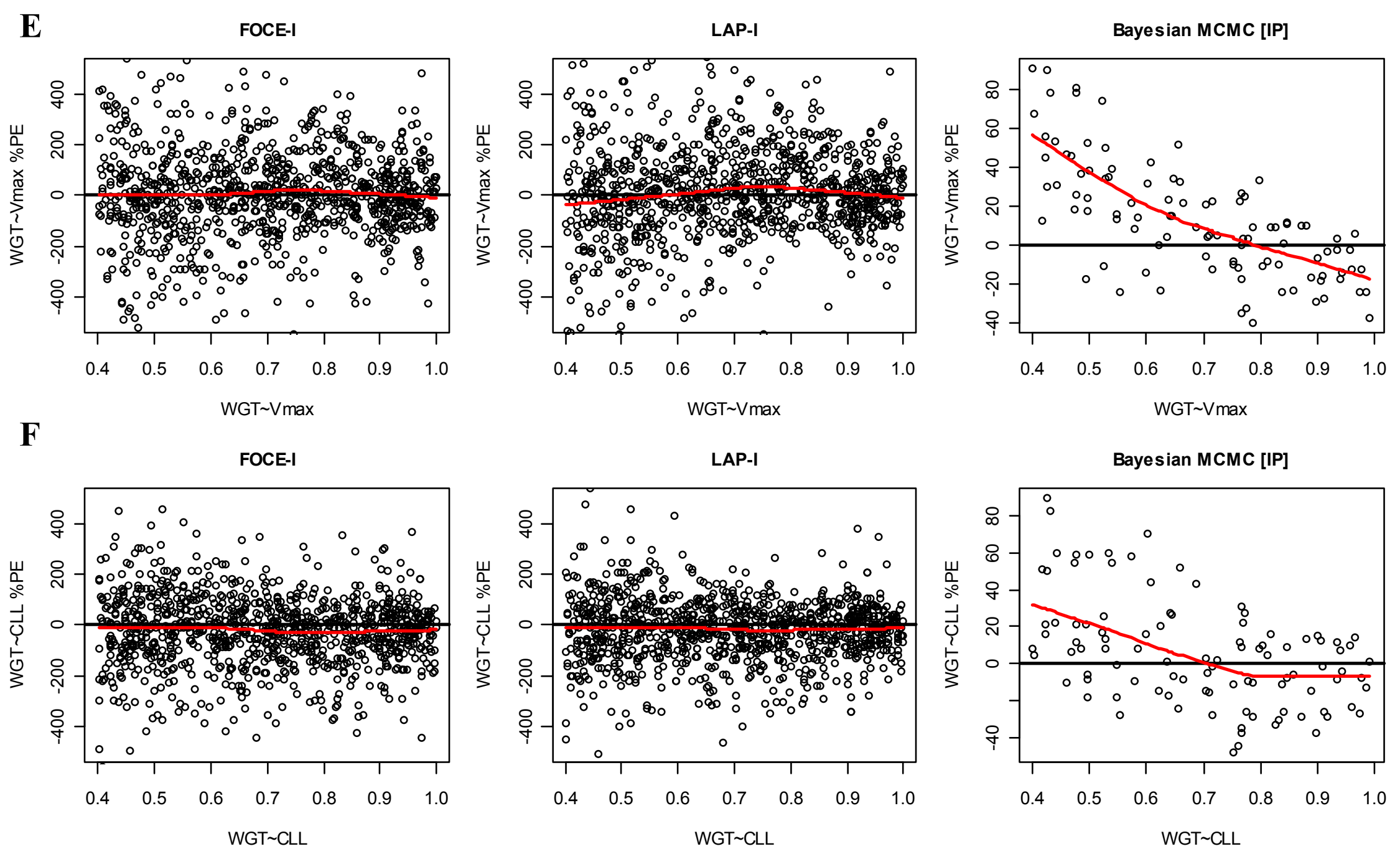

Figure 4-7 continued. 

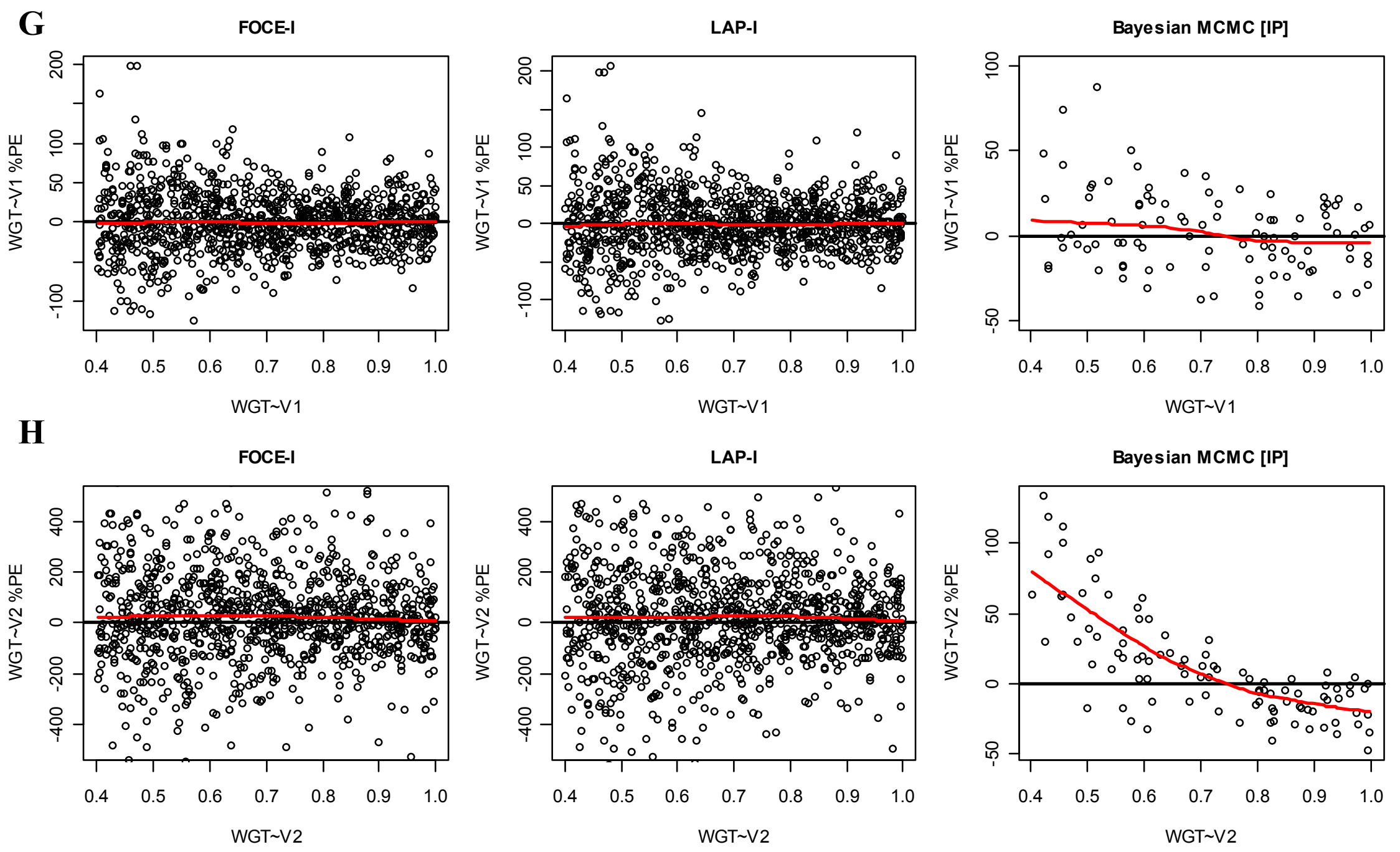

Figure 4-7 continued. 
Predictive checks showed that the parameter biases observed under the uninformative $600 \mathrm{mg}$ dose level study design for FOCE-I, LAP-I, and Bayesian MCMC with informative priors (Table 4-3) can result in predictions that differ significantly from the true model depending on the doses (concentrations) at which the predictions are made (Figures 4-8 through 4-10). For the true and biased parameter values, 1000 data sets were simulated using an uninformative study design template with different dose levels of 50,100, 200,400,600, or $800 \mathrm{mg}$, and the simulated concentrations during the full concentration-time profile at week 4 of therapy were then compared. Although the parameter values used for the simulations were based on the biases observed under the uninformative $600 \mathrm{mg}$ dose level study design, the predictive checks were carried out at other doses in addition to $600 \mathrm{mg}$ to investigate how the analysis-derived predictions would perform when extrapolated to other dose levels. For FOCE-I, LAP-I, and Bayesian MCMC with informative priors, the analysis-derived median and $90 \%$ prediction interval for concentrations during week 4 were quite similar to the predictions based on the true model at dose levels of $400 \mathrm{mg}$ and greater. At the lower dose levels $(50,100$, and $200 \mathrm{mg})$, the analysis-derived median and $90 \%$ prediction interval under each estimation method for the peak concentration at week 4 were very similar to the true model prediction. This can be expected given that the peak concentrations are largely determined by $V_{1}$, and the estimation methods produced negligible bias in both $V_{1}$ and the between-subject variance for $\mathrm{V}_{1}$. At the lower dose levels, the FOCE-I and LAP-I derived predictions for concentrations later in week 4 (e.g., the trough concentration) began to deviate from the true model, while the predictions based on Bayesian MCMC with informative priors remained closer to the true model. The trough concentrations are largely determined by the total clearance of the $\mathrm{mAb}$, which in the parallel elimination PK model, is determined by the clearance from the nonlinear pathway $\left(\mathrm{CL}_{\mathrm{NL}}\right)$ plus that of the linear pathway $\left(\mathrm{CL}_{\mathrm{L}}\right)$. A large bias was observed for $\mathrm{K}_{\mathrm{m}}$ under FOCE-I and LAP-I of $129 \%$ and $173 \%$, respectively, while the use of informative priors resulted in a much smaller bias of $34 \%$. These differences in the bias for $\mathrm{K}_{\mathrm{m}}$ were likely the main reason for the predictive check observations, given that for each estimation method much smaller biases were observed for parameters related to $\mathrm{V}_{\max }$ and $\mathrm{CL}_{\mathrm{L}}$ compared to $\mathrm{K}_{\mathrm{m}}$. An overprediction of $\mathrm{K}_{\mathrm{m}}$ would cause a decrease in $\mathrm{CL}_{\mathrm{int}}$, and consequently the $\mathrm{CL}_{\mathrm{NL}}$ and total clearance would decrease over the range of concentrations in which the nonlinear pathway is not saturated. This decrease in total clearance would result in overprediction of trough concentrations which is what was observed in Figures 4-8 and 4-9 for the FOCE-I and LAP-I derived predictions.

The Bayesian MCMC method with uninformative priors was not investigated under the uninformative $600 \mathrm{mg}$ dose level study design, because all of the initial estimation runs under this scenario failed to run to completion. This could be expected, as the uninformative nature of the data sets simulated under the $600 \mathrm{mg}$ dose level study design may not have supported the use of the vague priors specified in this study. However, if the originally specified vague priors were constrained to more biologically plausible values, but enough uncertainty retained so the priors could still be considered weakly informative, then the estimation runs would run to completion. The use of the biologically plausible but weakly informative priors was not further investigated in the 
$50 \mathrm{mg}$

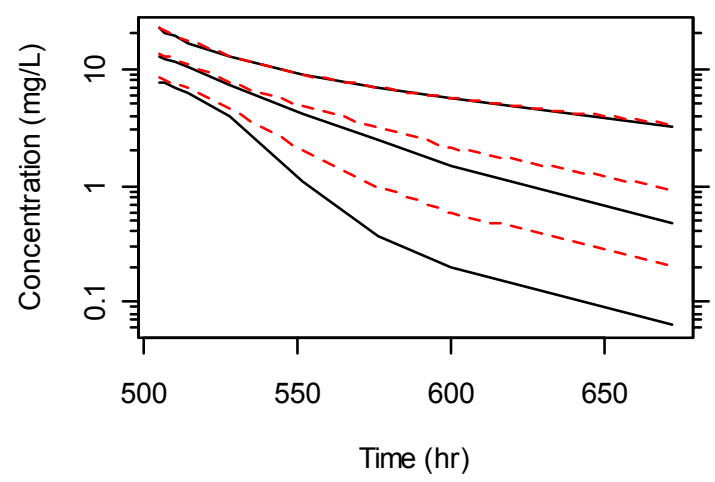

$200 \mathrm{mg}$

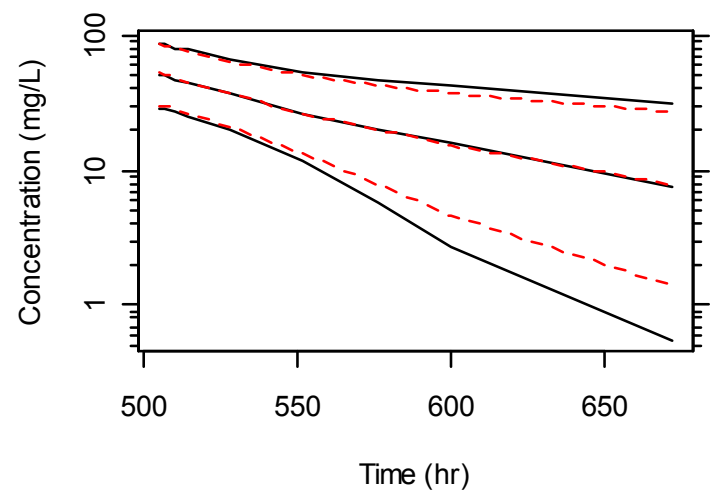

$600 \mathrm{mg}$

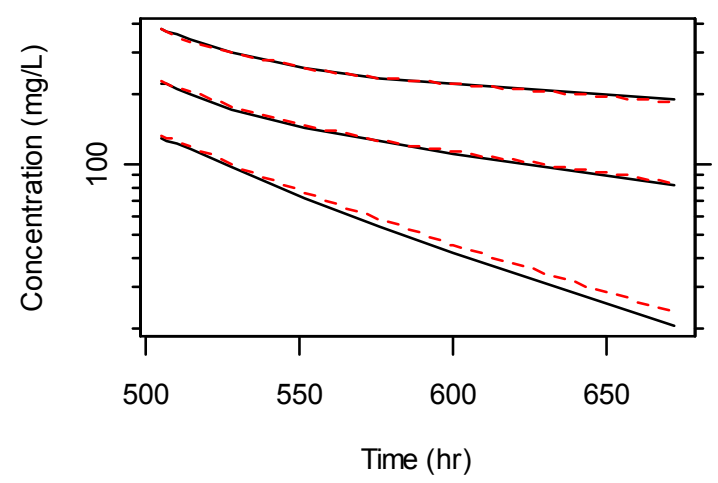

$100 \mathrm{mg}$
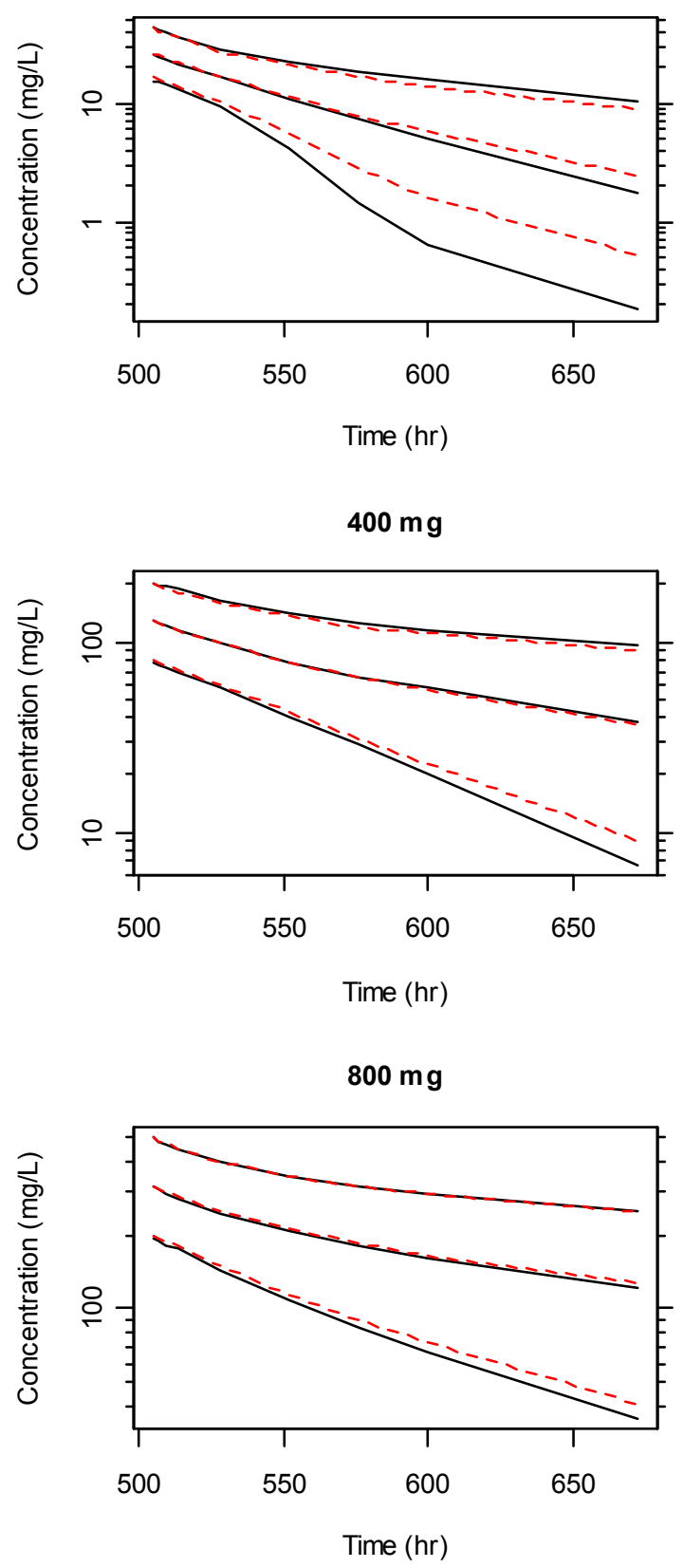

Figure 4-8. Simulated prediction intervals for concentrations during week 4 of therapy based on the true parameter values and biased parameter values derived from the FOCE-I results under the uninformative $600 \mathrm{mg}$ dose level study design. The true model and analysis-derived predictions are indicated by the solid black lines and dashed red lines, respectively. The three lines represent the 5th, 50th, and 95th percentiles determined from 1000 simulated data sets. The simulations were carried out at different dose levels using an uninformative study design template data set. 

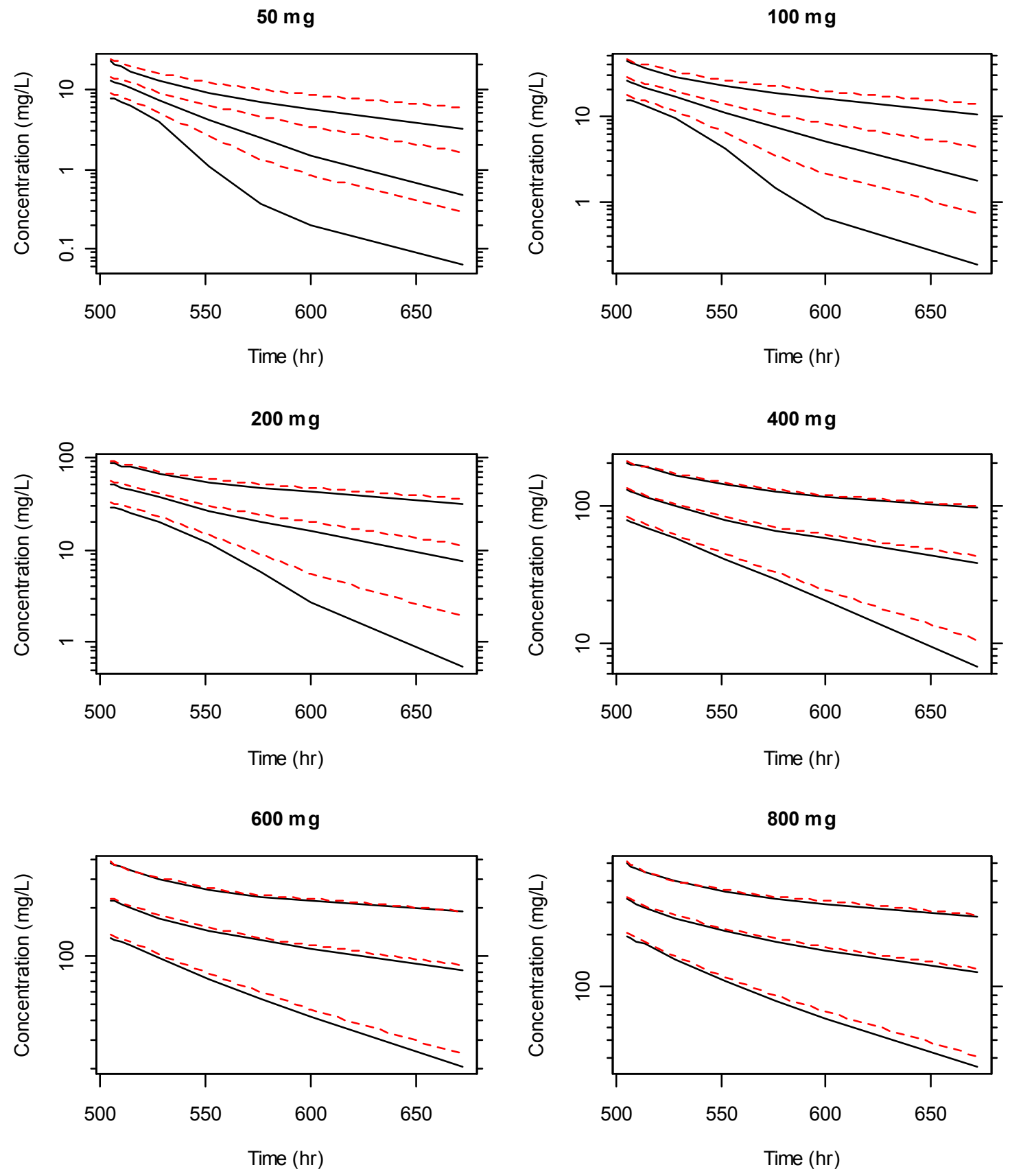

Figure 4-9. Simulated prediction intervals for concentrations during week 4 of therapy based on the true parameter values and biased parameter values derived from the LAP-I results under the uninformative $600 \mathrm{mg}$ dose level study design. The true model and analysis-derived predictions are indicated by the solid black lines and dashed red lines, respectively. The three lines represent the 5th, 50th, and 95th percentiles determined from 1000 simulated data sets. The simulations were carried out at different dose levels using an uninformative study design template data set. 
$50 \mathrm{mg}$

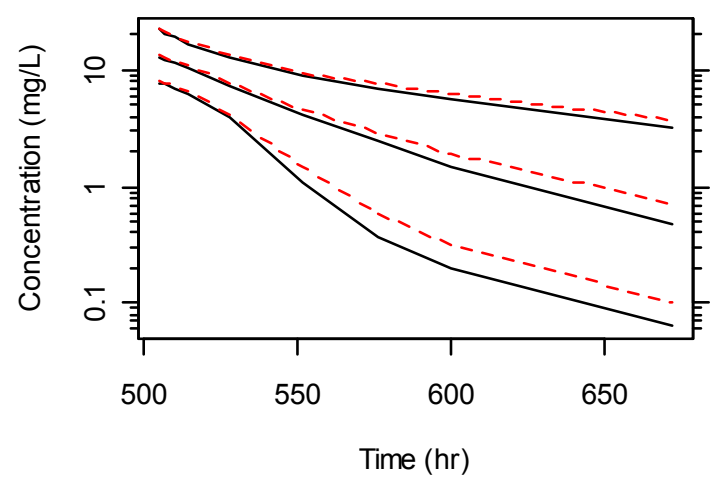

$200 \mathrm{mg}$

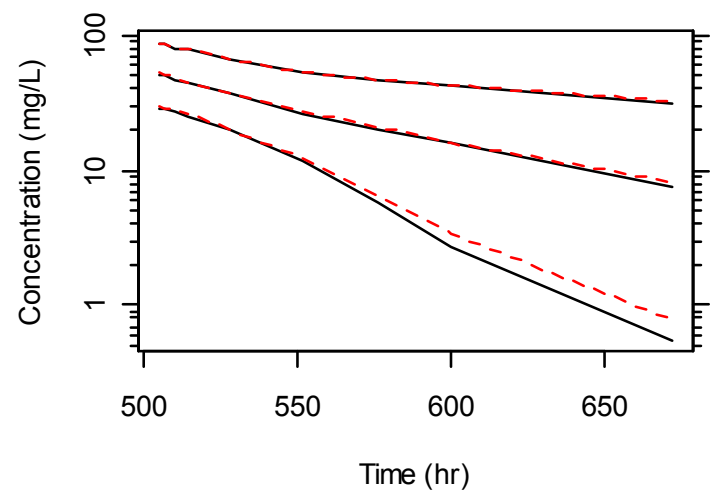

$600 \mathrm{mg}$

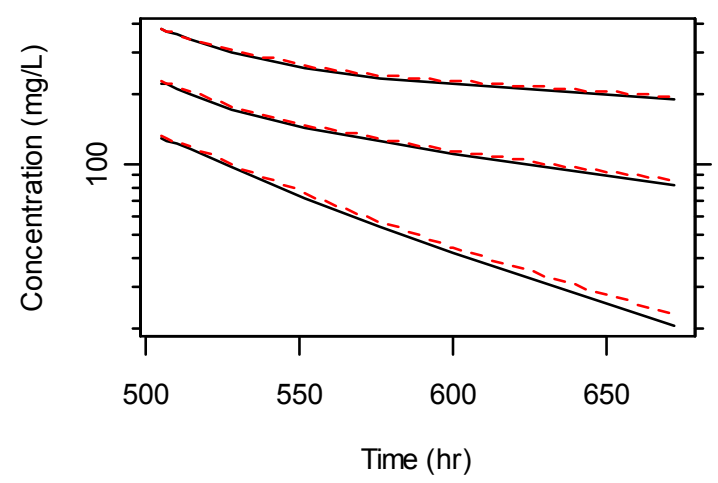

$100 \mathrm{mg}$

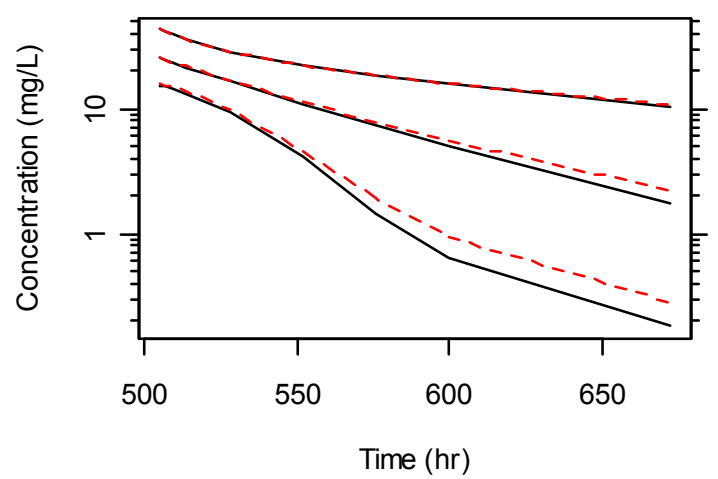

$400 \mathrm{mg}$

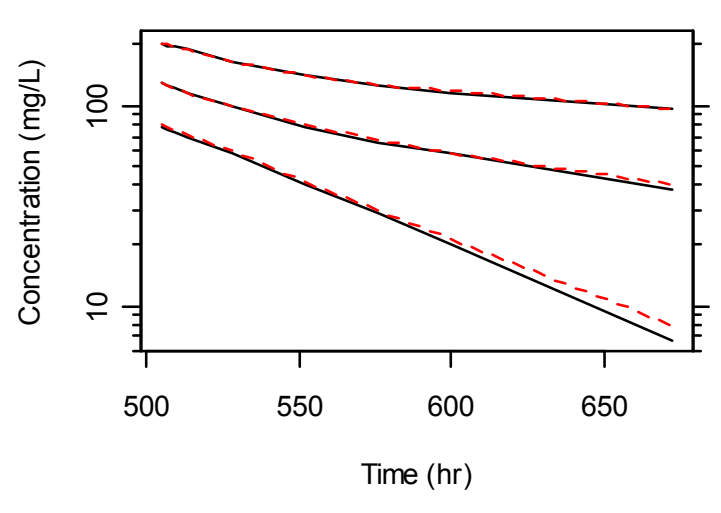

$800 \mathrm{mg}$

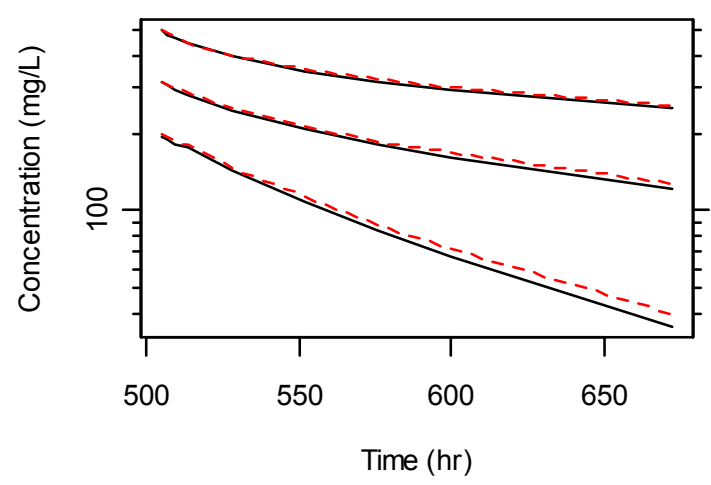

Figure 4-10. Simulated prediction intervals for concentrations during week 4 of therapy based on the true parameter values and biased parameter values derived from the Bayesian MCMC (with informative priors) results under the uninformative $600 \mathrm{mg}$ dose level study design. The true model and analysis-derived predictions are indicated by the solid black lines and dashed red lines, respectively. The three lines represent the 5th, 50th, and 95th percentiles determined from 1000 simulated data sets. The simulations were carried out at different dose levels using an uninformative study design template data set. 
study in order to maintain consistency in the methodology, since these priors would have differed from the vague priors used under the informative study design.

\section{Discussion}

Nonlinear elimination is a common characteristic of the PK of therapeutic mAbs $(6,8)$, and accordingly, PK models with nonlinear elimination were used in almost half of the population PK analyses of therapeutic mAbs reported in the literature (Table 1-1). It was also common in the population PK analyses to use a two-compartment model to describe the distribution of the therapeutic $\mathrm{mAb}$. It is estimated that half of all current projects in new drug development are biologics, with mAbs being the predominant class of biologics under clinical study (1). As the number of therapeutic mAbs undergoing drug development and gaining FDA approval continue to increase, it is important that currently available estimation methods used for population PK modeling be evaluated when applied to therapeutic mAbs with nonlinear PK. The challenge with detecting and characterizing nonlinear PK is not only dependent on the study design, but also on the estimation method used for the population PK analysis (163). However, little work has been done evaluating population estimation methods using PK models that are representative of the typical disposition characteristics of therapeutic mAbs, as most method comparison studies used a one-compartment model with linear elimination for comparison (142-147). In the current study, I evaluated the FO, FOCE-I, and LAP-I methods in NONMEM and a full Bayesian MCMC method in WinBUGS with both uninformative and informative priors in population PK modeling of therapeutic mAbs with nonlinear PK. The structural PK model used for the simulation of trial replicates was a two-compartment model with parallel linear and nonlinear elimination pathways, which was also used in the population PK analyses of several therapeutic mAbs (Table 1-1). Furthermore, to my knowledge this is the first study to have systematically evaluated the formal inclusion of prior information with Bayesian MCMC methods when used for population PK modeling.

Given the similar population PK characteristics of therapeutic mAbs (Chapter 1), the idea was that the published findings from the population PK studies could not only be used to define the parameter uncertainty distributions for simulation of the trial replicates, but could also be used as prior information in a Bayesian analysis. With regards to the simulation parameter uncertainty distributions, it was important that the values sampled from the distributions were realistic and representative of the population PK parameters observed for therapeutic mAbs in order to simulate concentration data for a prototypical $\mathrm{mAb}$. As shown in Figure 4-11, the probability density plots for the parameter uncertainty distributions adequately characterized the parameter estimates reported in published population PK analyses of therapeutic mAbs. Population estimates for $\mathrm{V}_{\max }$ and $\mathrm{K}_{\mathrm{m}}$ are not shown in Figure 4-11 for their respective uncertainty distributions since both parameters are specific for a given $\mathrm{mAb}$ and pharmacological target, and as

discussed in Chapter $1, \mathrm{~V}_{\max }$ and $\mathrm{K}_{\mathrm{m}}$ varied considerably among the different therapeutic mAbs. The probability density plots for the Bayesian informative prior distributions (not shown) differed somewhat from those for the simulation uncertainty distributions, as the 
Figure 4-11. Probability density plots of the parameter uncertainty distributions used for simulation of the trial replicates. The red vertical dashes represent parameter estimates reported in published population PK analyses of therapeutic mAbs (see Tables 1-3 through 1-6). In some of the plots the red dashes are staggered for visualization purposes. $\mathbf{S D}=$ standard deviation. 

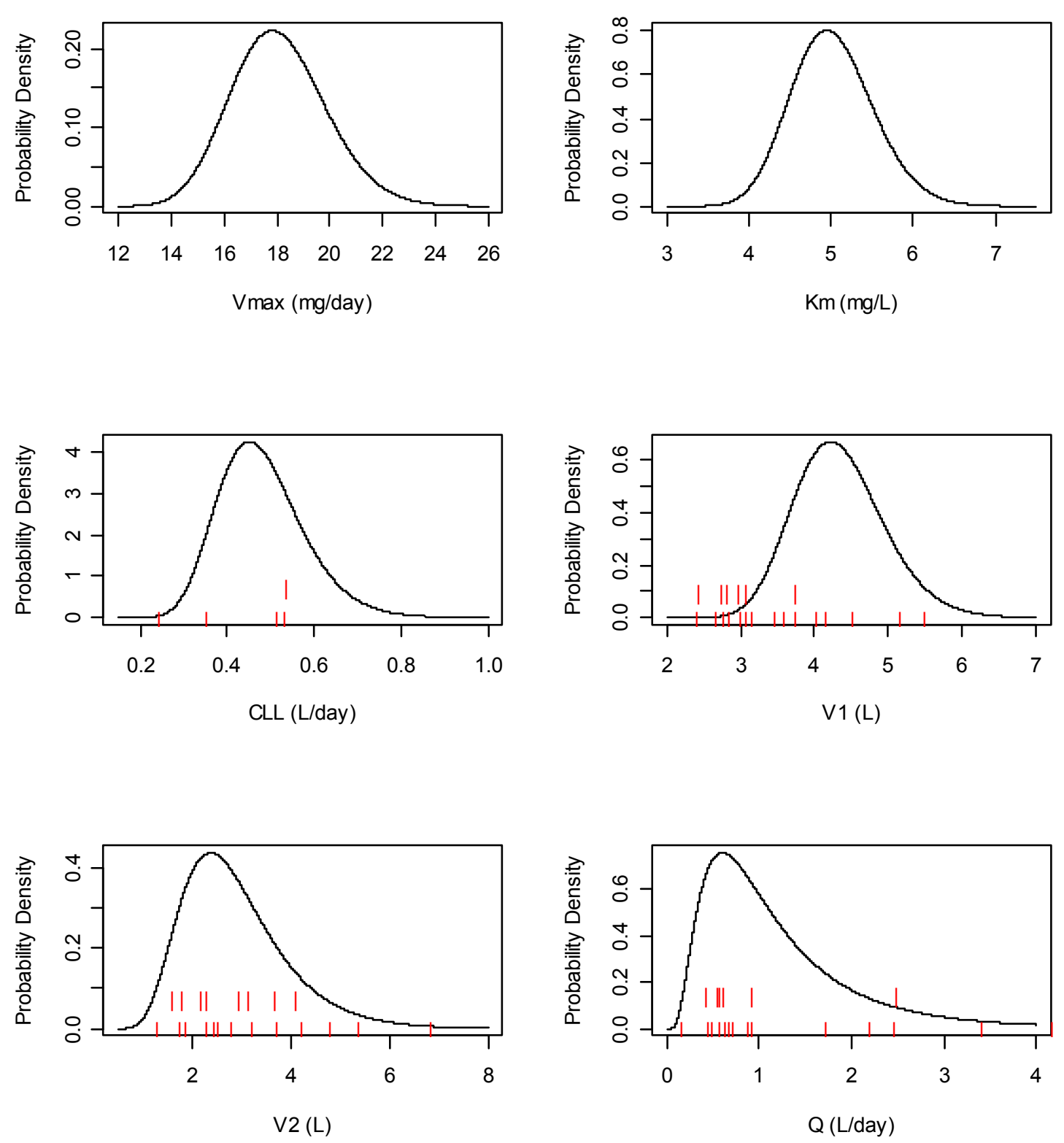

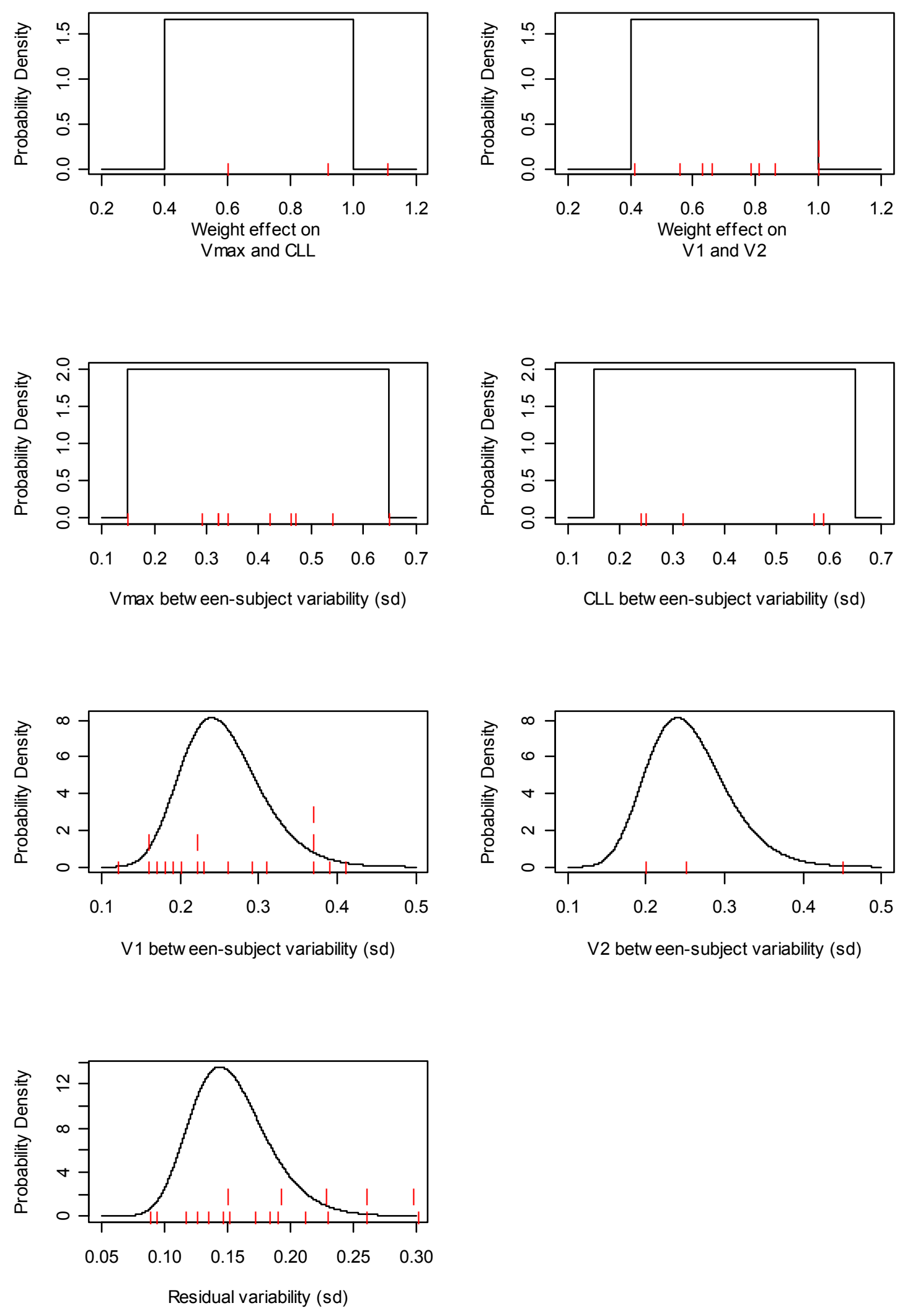

Figure 4-11 continued. 
Bayesian informative priors were updated with additional population PK studies of therapeutic mAbs that were published or found in the literature after the simulation of the replicate data sets had been performed. However, the Bayesian informative priors still adequately described the population PK parameter estimates reported in the literature for therapeutic mAbs. Figure 4-12 shows a scatterplot matrix of parameter values drawn from the multivariate lognormal simulation uncertainty distribution defined for the parameters $\mathrm{CL}_{\mathrm{L}}, \mathrm{V}_{1}, \mathrm{~V}_{2}$, and $\mathrm{Q}$, so that the correlations between the parameters can be visualized.

An issue that had to be addressed during this study was how to compare the parameter estimation output from NONMEM and WinBUGS. While NONMEM provides maximum likelihood estimates (i.e., point estimates), WinBUGS provides the full posterior marginal distributions of the MCMC sampled values of the model parameters. Summary statistics (e.g., mean, median, standard deviation, and 2.5 and 97.5 percentiles) and probability density plots of the posterior marginal distributions can then be obtained. Consideration was given to using the posterior mean, median, or mode, as the point estimate for calculating bias and precision of the parameter estimates for the Bayesian MCMC method. Since the maximum likelihood estimate is the mode of the likelihood, the closest analog in a Bayesian analysis to a maximum likelihood estimate would be the mode of the posterior distribution. However, estimating the mode from MCMC samples of a continuous variable can be problematic, and is not always reliable as this requires the probability density of the posterior distribution to be estimated from the MCMC samples and then the mode of the estimated density is used. For a symmetrical posterior distribution, the mean, median, and mode would all be approximately equal; however, for many of the model parameters a positively skewed posterior distribution was observed. Given the skewed nature of the posterior distributions, the posterior mean was typically larger than the mode and median. Therefore, for purposes of comparing the parameter estimation performance of WinBUGS and NONMEM, the posterior median was selected as a comparable metric to the maximum likelihood estimates provided by NONMEM. The posterior median was also used in a study by Bennett and Wakefield, in which the estimation performance of a Bayesian MCMC method in the software package POPKAN was compared to the FOCE method in NONMEM and a two-step algorithm within another software package PPHARM (142).

Another issue that had to be addressed in this study was that due to prolonged run times it was not feasible to evaluate the estimation performance of WinBUGS under each of the dose levels used for the uninformative study design. For this reason, WinBUGS was evaluated only under the $600 \mathrm{mg}$ dose level uninformative design. This dose level was selected for the reason that if the theoretical $\mathrm{mAb}$ in this study was undergoing drug development, a similar dose might be selected for entering phase II studies. The approach to selecting the $600 \mathrm{mg}$ dose level was to first simulate one replicate data set under the informative study design using the means and midpoints of the simulation parameter uncertainty distributions. A noncompartmental analysis was then performed on the simulated concentration-time data. As shown in Figure 4-13, there is a dosedependent decrease in clearance, which can be expected given the nonlinear PK of the 

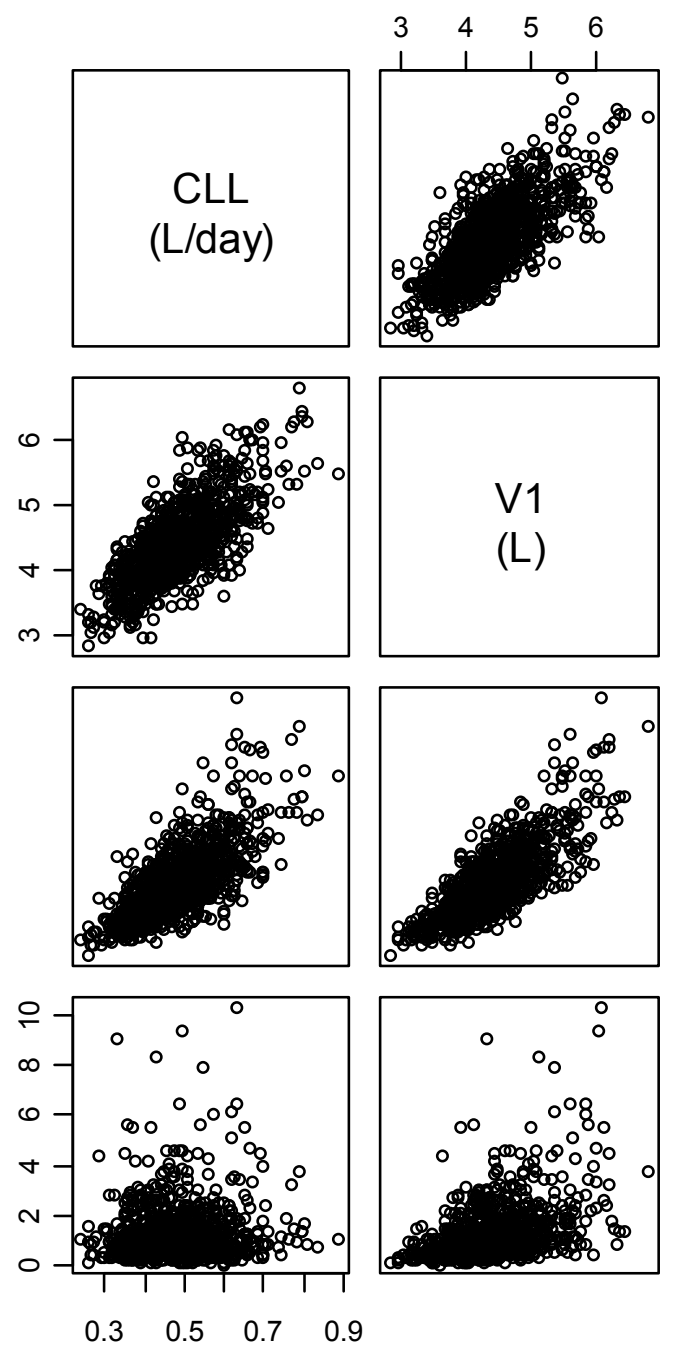
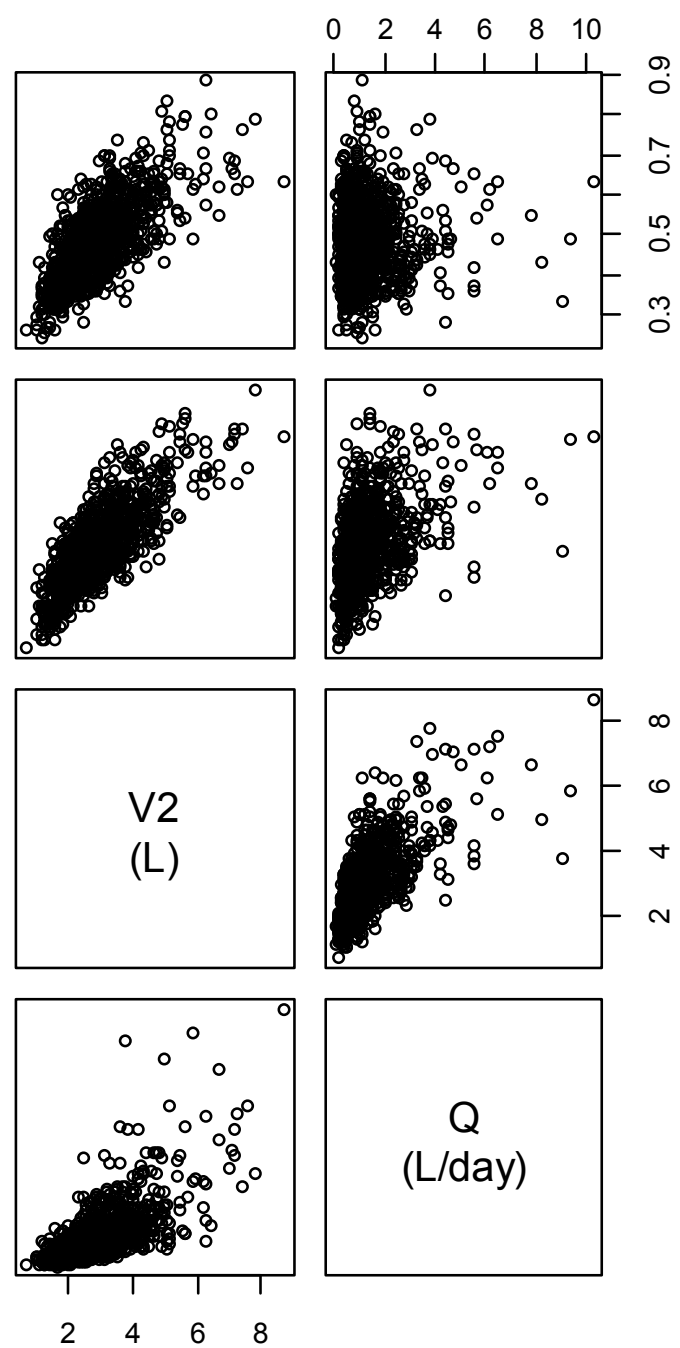

Figure 4-12. Scatterplot matrix of parameter values drawn from the multivariate lognormal uncertainty distribution defined for the parameters CLL, V1, V2, and Q, for simulating the trial replicates. 1000 values were drawn for each parameter. 


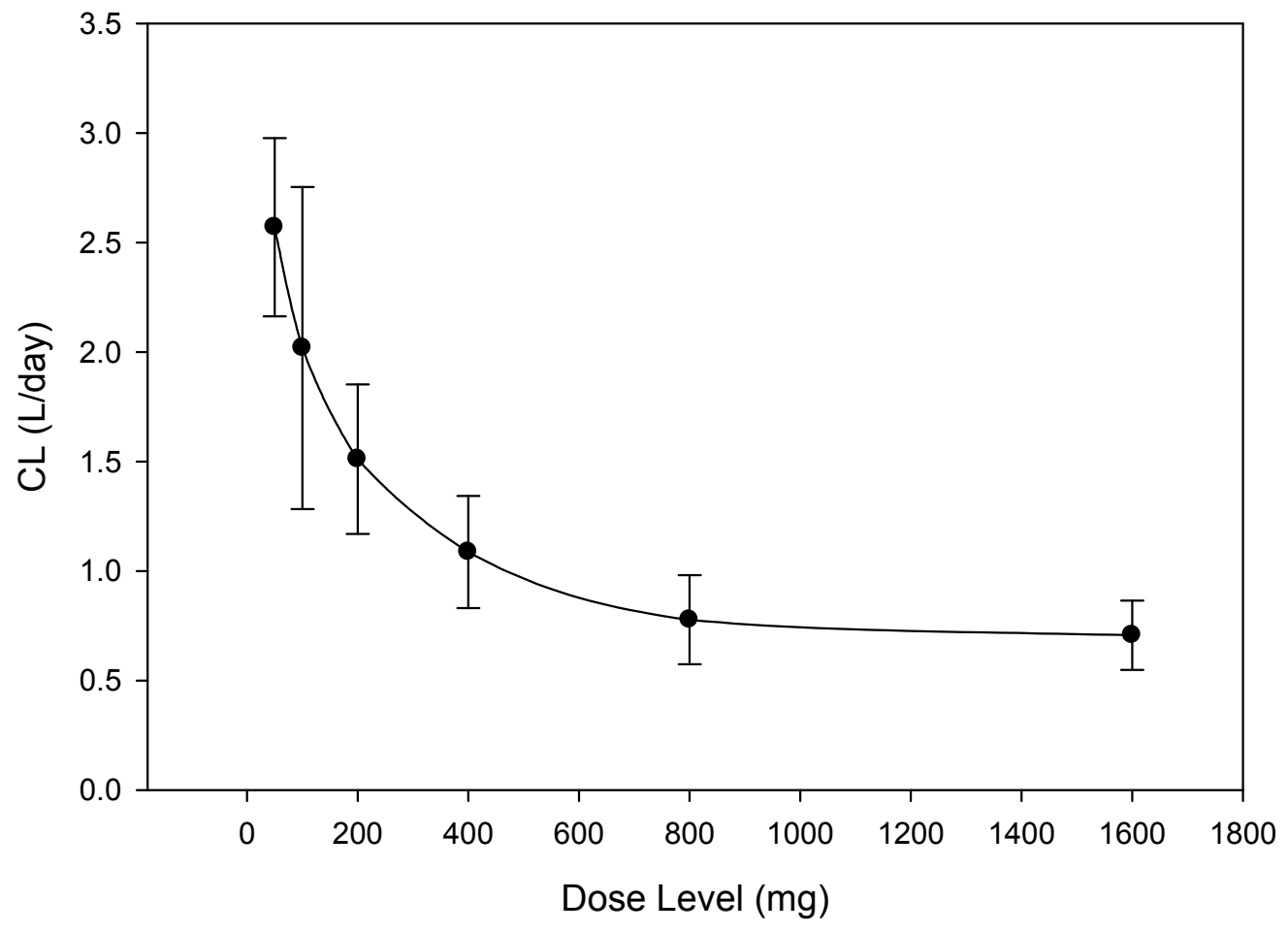

Figure 4-13. Plot of clearance versus dose based on a noncompartmental analysis of a simulated replicate dataset under the informative study design. There were six patients per dose level, and for each dose level the mean \pm SD clearance is shown. 
theoretical $\mathrm{mAb}$. Assuming that the theoretical $\mathrm{mAb}$ is eliminated via a saturable targetmediated mechanism (i.e., receptor-mediated endocytosis), then theoretically, the asymptotic decrease of the clearance of the $\mathrm{mAb}$ would be the result of saturating the pharmacologic target. Looking at Figure 4-13, the clearance of the mAb begins to level off somewhere around the $600 \mathrm{mg}$ dose level. These findings could be used as the basis for recommending a dose of around $600 \mathrm{mg}$ for future phase II studies. This rationale was similar to that used in a phase I study of cetuximab, where the maintenance dose recommendation for future phase II studies was based on the results of a noncompartmental analysis of cetuximab concentration-time data from patients treated at different dose levels (81). In the cetuximab phase I study, a maintenance dose of around $200 \mathrm{mg} / \mathrm{m}^{2}$ was recommended based on an observed dose-dependent decrease of cetuximab clearance over a range of $20-400 \mathrm{mg} / \mathrm{m}^{2}$, where saturation of the clearance began to occur around the $200 \mathrm{mg} / \mathrm{m}^{2}$ dose level (81). The $600 \mathrm{mg}$ dose level was also selected, because at larger doses the two-compartment parallel linear and nonlinear elimination model essentially collapses to a two-compartment linear model. At these higher doses it would be difficult for any estimation method to detect the nonlinear PK. This was indicated by the increasing bias in the population estimate for $\mathrm{K}_{\mathrm{m}}$ for the NONMEM estimation methods as the dose levels increased under the uninformative design (Tables 4-1, 4-3, C-1).

Under all study designs, the FO method generally produced larger bias and lower precision for the model parameters compared to the other estimation methods evaluated. This was expected since FO is generally considered to be a less accurate estimation method compared to FOCE and LAP (13). All three methods in NONMEM (FO, FOCE, LAP) linearize the nonlinear mixed effects model (e.g., population PK model) around the between-subject random effects $(112,113)$. The FO method takes a first-order approximation around the expected value of the between-subject random effects which is 0 (i.e., $\eta=0$ ). On the other hand, FOCE takes a first-order approximation around the conditional estimate of the between-subject random effects. The LAP method is similar to FOCE, but takes a second-order approximation. Despite the more accurate approximation of the nonlinear mixed effects model used by LAP, the estimation performance of LAP and FOCE were very similar under the informative and uninformative $600 \mathrm{mg}$ study designs (Tables 4-1 through 4-4), although the LAP method did show improvement over FOCE under the uninformative $600 \mathrm{mg}$ study design for the bias in $\mathrm{V}_{\max }$ and the between-subject variance for $\mathrm{V}_{\max }$ and $\mathrm{V}_{2}$. A detailed discussion of the theory behind the approximate maximum likelihood methods in NONMEM (FO, FOCE, LAP) and Bayesian MCMC methods, such as that used by WinBUGS, is not within the scope of this text but are discussed elsewhere $(112,113,115,116,150,152)$. Although, it is worth noting that Bayesian MCMC methods do not rely on analytical approximations of the nonlinear mixed effects model like with FO, FOCE, and LAP, but instead use Monte Carlo integration techniques to obtain parameter estimates for the exact model $(115,116)$. The extent to which the theoretical differences in the methods in NONMEM and WinBUGS translated into differences in their estimation performance is unknown and was not evaluated per se in this study. 
There are a number of practical advantages and disadvantages with NONMEM and WinBUGS. One advantage with Bayesian MCMC methods is the ability to include prior information in the population $\mathrm{PK}$ analysis by using informative priors. This advantage was demonstrated by the superior performance of Bayesian MCMC with informative priors over the NONMEM estimation methods under the uninformative 600 mg design. Another advantage with Bayesian MCMC is that full posterior distributions of the PK model parameters are provided from which inferences can made using the entire distribution. Depending on the computing resources available, a potential disadvantage with WinBUGS is prolonged run times. As observed in this study, run times with WinBUGS were considerably longer compared to when the NONMEM estimation methods were used. Average run times for FOCE-I and LAP-I under the informative study design were 7 minutes and 30 minutes, respectively, while run times for Bayesian MCMC with uninformative priors and informative priors averaged around 19 hours and 25 hours, respectively. Under the uninformative $600 \mathrm{mg}$ study design, average run times for FOCE-I, LAP-I, and Bayesian MCMC with informative priors were 8 minutes, 45 minutes, and 19 hours, respectively. In the past, another drawback to Bayesian MCMC methods was the lack of accessibility to such methods and software without having to do additional custom programming to implement the population PK model and to input and manage data. However, accessibility to Bayesian MCMC methods for population PK modeling is improving. For example, the BUGSModelLibrary (157), a recently developed WinBUGS PK/PD model library, was used in this study to implement the population PK model. In addition, a Bayesian MCMC method was incorporated in the recent release of NONMEM version VII (164). A benefit to using NONMEM is that it is the most widely used software for population PK/PD modeling (151), and since it has been available for more than 30 years, there is a wealth of studies and experience with using NONMEM in the literature. For pharmacometricians that have primarily used NONMEM for population PK/PD modeling, switching to WinBUGS may require additional training and education in Bayesian analysis and MCMC methods.

The NONMEM user's guide points out that the FO, FOCE, and LAP estimation methods can be inaccurate when dealing with highly nonlinear models (112). However, when evaluating the performance of any estimation method, one should always take into consideration the nature of the data and study design under which the method was evaluated. As shown in this study, the FOCE and LAP methods performed reasonably well and on par with the Bayesian MCMC method under the conditions used for the informative study design. FOCE and LAP also performed relatively well under the uninformative $200 \mathrm{mg}$ study design, as the bias and precision for many of the parameters were comparable to those under the informative study design, although a noticeably larger bias and/or lower precision was observed for some of the parameters (e.g., $\mathrm{V}_{2}$ and weight effect on $\mathrm{V}_{2}$ ) under the uninformative $200 \mathrm{mg}$ design (Tables 4-1, 4-2, C-1, C-2).

The estimation performance of FOCE-I and LAP-I decreased as the dose levels used for the uninformative study design $(200,500,600$, and $800 \mathrm{mg}$ ) were increased (Tables 4-3, 4-4, C-1, C-2). This decrease in performance was particularly evident for the model parameters related to nonlinear elimination. This was not a surprise, since the 
larger doses resulted in concentrations well above the $\mathrm{K}_{\mathrm{m}}$, thereby leading to saturation of the nonlinear elimination pathway and making it more difficult to detect and characterize the nonlinear PK. For this reason, I was interested in whether or not the nonlinearity could be detected with FOCE-I and LAP-I at the dose levels used for the uninformative design. In other words, would a two-compartment model with parallel nonlinear and linear elimination be selected over a more parsimonious two-compartment model with linear elimination at these dose levels? This question was investigated by fitting both PK models to 50 replicate data sets simulated under the uninformative study design for the 200, 500, 600, and $800 \mathrm{mg}$ dose levels. This was only done for the FOCE-I method, since it was believed that similar results would be achieved using LAP-I. Model selection was based on the likelihood ratio test. Since, the PK models are not strictly hierarchical, a $\mathrm{p}$ value of 0.01 was used which corresponds to a change in the OFV of 13.28 at 4 degrees of freedom. In more than $70 \%$ of the runs under the 200,500 , and 600 $\mathrm{mg}$ dose levels, the two-compartment parallel elimination model would have been selected over the two-compartment linear model. Under the $800 \mathrm{mg}$ dose level, this would have been the case in less than $30 \%$ of the runs. The significance of this finding is that while FOCE-I in NONMEM was able to detect nonlinearity at the $600 \mathrm{mg}$ dose level, and that it is plausible that a two-compartment parallel elimination model would have been selected over a simpler PK model, the results in Tables 4-3 and 4-4 show that FOCE-I at the $600 \mathrm{mg}$ dose level resulted in biased and imprecise estimates for many of the model parameters. This scenario is also relevant, because it represents a situation often encountered in population PK modeling where a more complex and mechanistically-plausible PK model is not fully supported by available concentration data due to study design limitations, and consideration must be given to using a more parsimonious PK model.

The global sensitivity analysis produced some interesting findings. Under the different study designs, the global sensitivity plots for the evaluated estimation methods showed that for most of the model parameters the percent estimation errors were insensitive to uncertainty in the values of the simulation parameters. However, FOCE-I, LAP-I, and Bayesian MCMC with uninformative priors tended to be more robust to uncertainty in the simulation values for select parameters (weight effect and the betweensubject variance for $\mathrm{V}_{\max }, \mathrm{CL}_{\mathrm{L}}$, and $\mathrm{V}_{2}$ ) compared to when informative Bayesian priors were used. The global sensitivity analysis results are conditional on the degree of uncertainty in the simulation parameter values employed in this study. Perhaps the FOCE-I and LAP-I methods would have been less robust had broader simulation uncertainty distributions been used for some of the parameters such as the betweensubject variances. However, the uncertainty distributions were defined so that the sampled parameter values were realistic and representative of those reported in published population PK analyses of therapeutic mAbs. Looking at the global sensitivity analysis plots under the informative study design for the weight effect and the between-subject variance for $\mathrm{V}_{\max }, \mathrm{CL}_{\mathrm{L}}$, and $\mathrm{V}_{2}$ (Figure 4-3), there were no trends in the estimation errors when uninformative priors were used, but there were when informative priors were used. This observation suggests that the formal inclusion of prior information influenced the estimates for these parameters, and it also illustrates the importance of investigating the 
sensitivity of parameter estimates to different prior distributions when performing a Bayesian analysis of population PK data.

The findings from this simulation study may be of value to pharmacometricians performing population $\mathrm{PK}$ analyses of therapeutic mAbs. The acceptable performance of the FOCE-I, LAP-I, and Bayesian MCMC estimation methods under the informative study design suggests that if sufficient concentration-time data is available to adequately characterize the nonlinear elimination of the $\mathrm{mAb}$, then any one of these estimation methods would be suitable for the population PK analysis. The choice then of one estimation method over another would depend on other factors such as available computing resources and familiarity with the methods. However, as often encountered in drug development, concentration-time data may only be available from clinical studies where limitations in the study design make it difficult to detect and characterize the nonlinear elimination of the therapeutic mAb. As discussed in Chapter 1, these clinical study design limitations are often due to the doses (concentrations) studied, because the doses (which may be clinically relevant) result in concentrations that are high enough to saturate the nonlinear target-mediated elimination of the therapeutic mAb. For example, nonlinear elimination has been observed for efalizumab and trastuzumab and attributed to saturable target-mediated elimination $(33,35,79,82)$, but population PK analyses of the two mAbs were able to adequately describe their PK using a compartmental model with linear elimination $(33,46)$. Based on PK data from dose escalation studies of efalizumab (35) and trastuzumab (82), the doses of these mAbs investigated in the aforementioned population PK analyses may have resulted in steady-state concentrations where targetmediated clearance was saturated, and thus the use of a PK model with linear elimination was warranted. In this study, the estimation methods were evaluated under similar conditions with the uninformative study designs where concentration data was only available from a single dose level, and the dose levels selected resulted in concentrations that were generally above the $\mathrm{K}_{\mathrm{m}}$. Under a less-than-optimal study design, such as the uninformative $600 \mathrm{mg}$ dose design, consideration should first be given to what the population PK model is to be used for. If the model is to be used for describing/predicting concentrations at doses where the nonlinear elimination pathway is at or near saturation, then using a PK model with linear elimination would be a reasonable approach as the nonsaturable Fc-mediated elimination pathway becomes the major determinant of the mAb clearance (Chapter 1, Figure 1-2B). In this case, the use of the FOCE or LAP estimation methods in NONMEM would likely be sufficient. However, if for example the population PK model is to be used to predict concentrations at lower doses, then the findings under the uninformative $600 \mathrm{mg}$ study design would suggest that one may want to consider the use of a Bayesian MCMC method with informative priors given its improved performance over the NONMEM estimation methods. As shown in Figures 4-8 through 4-10, the analysis-derived predictions for FOCE-I, LAP-I, and Bayesian MCMC with informative priors were quite similar to the predictions based on the true model at the $600 \mathrm{mg}$ dose level. However, at lower doses, the analysis-derived predictions for FOCE-I and LAP-I deviated further from the true model than the analysis-derived predictions for Bayesian MCMC with informative priors. As discussed in Chapter 1 and implemented in this study, the findings from published population $\mathrm{PK}$ analyses of therapeutic $\mathrm{mAbs}$ could be used as prior information given 
their similar population PK characteristics. Prior information on the PK of the therapeutic $\mathrm{mAb}$ of interest could also be available and used in defining the priors.

The population PK analysis of cetuximab in Chapter 3 found that the PK of cetuximab was best described using a two-compartment model with nonlinear elimination. The final population PK model was able to adequately describe the nonlinear PK of cetuximab at the clinically approved dosage regimen, and the PK parameter estimates were in line with a previous population PK analysis of cetuximab conducted for regulatory purposes which also used the same structural model as in my study $(109,140)$. The population estimates for $\mathrm{V}_{\max }$ and $\mathrm{K}_{\mathrm{m}}$ were also in agreement with findings from other PK analyses of cetuximab $(123,139)$. Concentration data from multiple dose groups was available in the cetuximab population PK analysis conducted for regulatory purposes $(109,140)$ and in the aforementioned cetuximab PK studies $(123$, 139). Various issues were encountered during the cetuximab analysis which were likely the result of attempting to describe its nonlinear elimination. This difficulty was likely due in part to limitations of the clinical study designs, which included the lack of dose escalation concentration data and that the majority of patients provided only peak and trough concentrations. Given the relative uninformativeness of the data that was available for the cetuximab population PK analysis (particularly the single dose level study designs), the results from the current study suggest that the inclusion of prior information within the context of a full Bayesian analysis may have benefited the cetuximab analysis. Prior information was available from a number of sources that could have been used in the cetuximab population PK analysis. For example, the results from the previously conducted population PK analysis of cetuximab could have been used $(109,140)$, and parameter estimates for $\mathrm{V}_{\max }$ and/or $\mathrm{K}_{\mathrm{m}}$ had also been reported in other PK studies of cetuximab $(80,123,139)$. In addition, it would have been a reasonable approach to have also used the published results from population PK analyses of other therapeutic mAbs as prior information in the cetuximab analysis.

In summary, I conducted a simulation study to compare the parameter estimation performance of the FO, FOCE-I, and LAP-I methods in NONMEM and a full Bayesian MCMC method in WinBUGS when applied to population PK modeling of therapeutic mAbs with nonlinear PK. The Bayesian MCMC method was evaluated with both uninformative and informative priors. The estimation methods were evaluated under a dose-ranging design and four different single dose level designs at different dose levels. When sufficient data was available to characterize the nonlinear PK of the therapeutic $\mathrm{mAb}$, as in the case of the dose-ranging design, the estimation performance of the FOCEI, LAP-I, and Bayesian MCMC (with both sets of priors) methods were comparable and demonstrated acceptable bias and precision of the parameter estimates. Under a lessthan-optimal study design, the formal inclusion of prior information with the Bayesian MCMC method provided a clear performance advantage over the other methods. 


\section{CHAPTER 5. SUMMARY OF DISSERTATION RESEARCH}

Over the past two decades, there has been an increase in the research and development and therapeutic application of mAbs. Currently, there are over $20 \mathrm{mAb}$ products (i.e. antibodies, antibody fragments, or antibody-conjugates) approved by the FDA for therapeutic use in areas such as oncology, immunology, ophthalmology, cardiovascular disease, and infectious disease. It is estimated that half of all current projects in new drug development are biologics, with mAbs being the predominant class of biologics under clinical study (1). In 2008, it was reported that there were over 200 mAbs undergoing clinical study (2). One likely reason for the continued success of the clinical development of mAbs is the application and integration of PK and PD concepts in all stages of pre-clinical and clinical drug development $(3,4)$. One application of $\mathrm{PK} / \mathrm{PD}$ concepts in drug development is population PK, which attempts to quantify the typical disposition characteristics and sources of PK variability (such as between-subject, within-subject, and inter-occasion variability) within study populations. Population PK also attempts to identify and quantify the impact of covariates on systemic drug exposure, and assess their potential implications for clinical dosing. The general theme of my dissertation research was population PK modeling of therapeutic mAbs, which focused on the population PK modeling of cetuximab (Chapter 3), and the evaluation of different estimation methods for population PK modeling of therapeutic mAbs with nonlinear PK characteristics (Chapter 4).

A large number of population PK analyses of therapeutic mAbs have been published in the scientific literature. In Chapter 1, I summarized the findings from these population PK studies and related the findings to the general PK and structural characteristics of therapeutic mAbs. A two-compartment model was used in the majority of the population analyses to describe the disposition of the mAb. Population estimates for $\mathrm{V}_{1}$ and $\mathrm{V}_{2}$ were typically small with a median (range) of $3.1 \mathrm{~L}(2.4-5.5)$ and $2.8 \mathrm{~L}$ (1.3-6.8), respectively. The estimated between-subject variability in $\mathrm{V}_{1}$ was usually moderate with a median (range) of $28 \% \mathrm{CV}$ (12-84). Between-subject variability in other distribution related parameters such as $\mathrm{V}_{2}$ and $\mathrm{Q}$ were often not estimated. Although the most frequent PK models used in the population analyses were models with linear elimination, other models with nonlinear or parallel linear and nonlinear elimination pathways were also applied as many therapeutic mAbs are eliminated via saturable target-mediated mechanisms. Population estimates of $\mathrm{V}_{\max }$ and $\mathrm{K}_{\mathrm{m}}$, the parameters characterizing Michaelis-Menten type saturable clearance pathways, varied considerably among the different therapeutic mAbs. However, estimates for $\mathrm{CL}_{\mathrm{T}}$ in $\mathrm{mAbs}$ with linear elimination characteristics and for $\mathrm{CL}_{\mathrm{L}}$ in $\mathrm{mAbs}$ with parallel linear and nonlinear elimination were quite similar among the different $\mathrm{mAbs}$, and typically ranged from around $0.2-0.5 \mathrm{~L} /$ day, which is relatively close to the estimated CL of endogenous IgG of $0.21 \mathrm{~L} /$ day. The between-subject variability in $\mathrm{V}_{\max }, \mathrm{CL}_{\mathrm{T}}$, and $\mathrm{CL}_{\mathrm{L}}$ was moderate to large with estimates ranging from $15-65 \% \mathrm{CV}$. Measures of body size were the most commonly identified covariates found to influence the PK of therapeutic mAbs. My literature review found that many characteristics of the population PK of currently used therapeutic mAbs are quite similar despite differences in their pharmacologic targets and 
studied patient populations. These similarities are likely in part due to general structural features that are shared among most therapeutic mAbs as IgG molecules such as a functional human $\mathrm{Fc}$ region. One could hypothesize that target-binding specific PK parameters such as $V_{\max }$ and $K_{m}$ are likely to be influenced by the interaction between a given therapeutic $\mathrm{mAb}$ and its target, and thus show high variability among different mAbs, while the differences among parameters such as $\mathrm{CL}_{\mathrm{L}}, \mathrm{V}_{1}, \mathrm{~V}_{2}$, and $\mathrm{Q}$ that are thought to be largely affected by the general structural features of mAbs are minimal. Given the similar population PK characteristics of therapeutic mAbs, I proposed in this review that the results from the population PK analyses could serve a practical purpose and be used to construct informative priors for a Bayesian data analysis or for constructing parameter uncertainty distributions to simulate PK data for a prototypical therapeutic $\mathrm{mAb}$. These ideas were put into practice in Chapter 4 where the estimation methods in NONMEM and WinBUGS were evaluated.

A therapeutic $\mathrm{mAb}$ that was of particular interest in my dissertation research was cetuximab, which is directed against EGFR and is indicated in the treatment of SCCHN. In Chapter 3, I performed a population PK analysis of cetuximab using nonlinear mixed effects modeling and the software NONMEM (version V). I hypothesized that nonlinear mixed effects modeling could be used to develop a population PK model that could adequately predict the systemic exposure of cetuximab in patients with SCCHN. Furthermore, I hypothesized that covariates could be identified that are significant predictors for cetuximab systemic exposure. A total of 912 cetuximab concentrations were available from 143 patients with recurrent and/or metastatic SCCHN enrolled in two phase I/II studies. The PK of cetuximab was best described by a two-compartment model with Michaelis-Menten type saturable elimination. Mean population estimates (betweensubject variability, $\% \mathrm{CV})$ of the $\mathrm{PK}$ parameters were: $\mathrm{V}_{\max } 4.38 \mathrm{mg} / \mathrm{hr}(15.4 \%), \mathrm{K}_{\mathrm{m}} 74$ $\mu \mathrm{g} / \mathrm{ml}, \mathrm{V}_{1} 2.83 \mathrm{~L}(18.6 \%), \mathrm{V}_{2} 2.43 \mathrm{~L}(56.4 \%)$, and Q $0.103 \mathrm{~L} / \mathrm{hr}(97.2 \%)$. Covariates that were identified included IBW and WBC count as predictors of $\mathrm{V}_{\max }$, and WGT as a predictor of $\mathrm{V}_{1}$. The findings of the population $\mathrm{PK}$ analysis suggest that clinical dose adjustments beyond the approved BSA-based dosing of cetuximab may be warranted in patients with extreme deviations of their actual body weight from IBW. Agreement between simulated and measured concentrations for up to 43 weeks of therapy indicated that the final population PK model was able to adequately describe the nonlinear PK of cetuximab in patients with SCCHN at the currently approved dosage regimen, and that the cetuximab PK parameters remained constant during prolonged therapy.

During the cetuximab population PK analysis, a number of issues and challenges were encountered which were likely the result of an attempt to describe the nonlinear elimination of cetuximab. The difficulty characterizing the nonlinear PK of cetuximab was likely due to limitations of the clinical study designs, which included the lack of dose escalation data and the majority of patients provided only peak and trough concentrations. However, the difficulty may also have been due to the use of the FOCE estimation method throughout the analysis. It is known that FOCE, in addition to the FO and LAP estimation methods in NONMEM, can be inaccurate when dealing with highly nonlinear models such as the model with nonlinear elimination used to describe the PK of cetuximab (112). Therefore, it was questioned whether or not the use of another 
population estimation method in the cetuximab analysis would have led to more accurate parameter estimates and better characterization of the nonlinear PK of cetuximab. I was particularly interested in how a full Bayesian analysis using MCMC methods such as that implemented in the software WinBUGS would have performed. Unlike the aforementioned estimation methods in NONMEM, Bayesian MCMC methods do not rely on analytical approximations of the nonlinear mixed effects model, but instead use Monte Carlo integration techniques to obtain parameter estimates for the exact model $(115,116)$. Bayesian MCMC methods also allow one to include prior information about the model parameters in the Bayesian hierarchical model by using informative priors.

The question of how well other population estimation methods would have performed in evaluating the nonlinear elimination of cetuximab is also relevant for other therapeutic mAbs. Nonlinear elimination is a common characteristic of the PK of therapeutic mAbs $(6,8)$, and accordingly, PK models with nonlinear elimination were used in almost half of the population PK analyses of therapeutic mAbs published in the scientific literature (Chapter 1). In addition, similar difficulties encountered during the cetuximab analysis were also reported in population PK analyses of other therapeutic mAbs (Chapter 1). The challenge with detecting and characterizing nonlinear PK is not only dependent on the study design, but also on the estimation method used for the population PK analysis (163). However, little work has been done so far evaluating population estimation methods using PK models that are representative of the typical disposition characteristics of therapeutic mAbs, as most method comparison studies used a one-compartment model with linear elimination for comparison (142-147).

I hypothesized that compared to the FO, FOCE-I, and LAP-I methods in NONMEM, other parameter estimation methods that avoid linearization of the nonlinear mixed effects model such as Bayesian MCMC, would lead to more accurate parameter estimates and better characterization of the nonlinear PK encountered with therapeutic mAbs such as cetuximab. I conducted a simulation study to compare the parameter estimation performance of the FO, FOCE-I, and LAP-I methods in NONMEM and a Bayesian MCMC method in WinBUGS when applied to population PK modeling of therapeutic mAbs with nonlinear PK. The Bayesian MCMC method was evaluated with uninformative and informative priors, which were obtained from the literature. To my knowledge, this was the first study to have systematically evaluated the formal inclusion of prior information with Bayesian MCMC methods when applied to population PK modeling. The uncertainty distributions for the population PK model parameters were also obtained from the literature in order to simulate concentration data for a prototypical therapeutic $\mathrm{mAb}$. The estimation methods were evaluated under a dose-ranging design (informative study design) and four different single dose level designs at different dose levels (uninformative study designs). In my analysis, I addressed the following three subhypotheses: 1) under all study designs the FO method would be less accurate and precise compared to the other estimation methods; 2) when sufficient data is available to characterize the nonlinear $\mathrm{PK}$ of the therapeutic $\mathrm{mAb}$, as in the case of the informative design, the estimation performance of FOCE-I, LAP-I, and Bayesian MCMC (with both sets of priors) would be adequate and comparable; and 3) under the less-than-optimal (uninformative) study designs there would be a decline in the estimation performance of 
the FOCE-I and LAP-I methods, while the formal inclusion of prior information with the Bayesian MCMC method would provide a clear performance advantage over the other methods.

Under all study designs, the FO method generally produced larger bias and lower precision for the model parameters compared to the other estimation methods. Comparison of the estimation performance of the methods in NONMEM and WinBUGS was limited to the informative and uninformative $600 \mathrm{mg}$ dose level study designs due to prolonged run times with WinBUGS. Under the informative study design, bias and precision for all model parameters was less than $\pm 25 \%$ and $52 \%$, respectively, for FOCEI, LAP-I, and Bayesian MCMC with both sets of priors. Under the uninformative $600 \mathrm{mg}$ dose level study design, the estimation performance of FOCE-I and LAP-I decreased as bias and precision for many of the model parameters, in particular those related to nonlinear elimination, significantly increased to $\pm 40-173 \%$ and $53-173 \%$, respectively, while Bayesian MCMC with informative priors produced results that were comparable to those under the informative study design.

Given the relative uninformativeness of the data that was available for the cetuximab population PK analysis, the results from the estimation method comparison study suggested that the inclusion of prior information within the context of a full Bayesian analysis may have benefited the cetuximab analysis. Prior information was available from a number of sources that could have been used in the cetuximab population PK analysis. For example, the results from a previously conducted population PK analysis of cetuximab could have been used $(109,140)$, and parameter estimates for $\mathrm{V}_{\max }$ and/or $\mathrm{K}_{\mathrm{m}}$ had also been reported in prior PK studies of cetuximab (80, 123, 139). In addition, it would have been a reasonable approach to have also used the published results from population $\mathrm{PK}$ analyses of other therapeutic mAbs as prior information given their similar population PK characteristics.

To visualize the potential application of the results from the estimation method comparison study, consider a population PK analysis of a prototypical therapeutic mAb that exhibits nonlinear PK due to saturable target-mediated elimination mechanisms. If sufficient concentration data is available to adequately characterize the nonlinear elimination of the mAb, then the adequate performance of the FOCE-I, LAP-I, and Bayesian MCMC estimation methods under the informative study design would suggest that any one of these estimation methods would be suitable for the population PK analysis. The choice then of one estimation method over another would depend on other factors such as available computing resources and familiarity with the methods. However, as frequently encountered in drug development, consider that concentration data is only available from a less-than-optimal study design such as the uninformative $600 \mathrm{mg}$ dose level design used in the method comparison study, where the dose results in concentrations that are high enough to saturate the nonlinear target-mediated elimination of the mAb. In this case, consideration should first be given to what the population PK model is to be used for. If the model is to be used for describing/predicting concentrations at doses where the nonlinear elimination pathway is at or near saturation, then using a PK model with linear elimination would be a reasonable approach as the 
nonsaturable Fc-mediated elimination pathway becomes the major determinant of the $\mathrm{mAb}$ clearance. In this case, the use of the FOCE or LAP estimation methods in NONMEM would likely be sufficient. However, if for example, the population PK model is to be used to predict concentrations at lower doses, then the findings under the uninformative $600 \mathrm{mg}$ study design would suggest that one may want to consider the use of a Bayesian MCMC method with informative priors given the superior estimation performance over the NONMEM estimation methods.

In summary, the general theme of my dissertation research was population PK modeling of therapeutic mAbs. My research work began with a population PK analysis of cetuximab in patients with SCCHN. The challenges and issues encountered during the analysis led me to investigate the estimation performance of the FO, FOCE-I, and LAP-I methods in NONMEM and a Bayesian MCMC method (with both uninformative and informative priors) in WinBUGS when applied to population PK modeling of therapeutic $\mathrm{mAbs}$ with nonlinear PK, and to delineate scenarios in which one estimation method may be superior to the others. The findings from this work should be of value to pharmacometricians who are involved in population PK modeling of therapeutic mAbs. 


\section{LIST OF REFERENCES}

1. Maggon KF. Monoclonal antibody "gold rush". Curr Med Chem 2007;14(18):1978-87.

2. Reichert JM. Monoclonal antibodies as innovative therapeutics. Curr Pharm Biotechnol 2008;9(6):423-30.

3. Galluppi GR, Rogge MC, Roskos LK, Lesko LJ, Green MD, Feigal DW, Jr., et al. Integration of pharmacokinetic and pharmacodynamic studies in the discovery, development, and review of protein therapeutic agents: a conference report. Clin Pharmacol Ther 2001;69(6):387-99.

4. Meibohm B. The Role of Pharmacokinetics and Pharmacodynamics in the Development of Biotech Drugs. In: Meibohm B, editor. Pharmacokinetics and Pharmacodynamics of Biotech Drugs: Principles and Case Studies in Drug Development. Weinheim, Germany: Wiley-VCH; 2006. p. 3-16.

5. Meibohm B, Derendorf H. Pharmacokinetic/pharmacodynamic studies in drug product development. J Pharm Sci 2002;91(1):18-31.

6. Kuester K, Kloft C. Pharmacokinetics of Monoclonal Antibodies. In: Meibohm B, editor. Pharmacokinetics and Pharmacodynamics of Biotech Drugs: Principles and Case Studies in Drug Development. Weinheim, Germany: Wiley-VCH; 2006. p. 45-92.

7. Lobo ED, Hansen RJ, Balthasar JP. Antibody pharmacokinetics and pharmacodynamics. J Pharm Sci 2004;93(11):2645-68.

8. Tabrizi MA, Tseng CM, Roskos LK. Elimination mechanisms of therapeutic monoclonal antibodies. Drug Discov Today 2006;11(1-2):81-8.

9. Wang W, Wang EQ, Balthasar JP. Monoclonal antibody pharmacokinetics and pharmacodynamics. Clin Pharmacol Ther 2008;84(5):548-58.

10. Roitt IM, Delves PJ, Martin SJ, Burton DR. Roitt's Essential Immunology. 11th ed. Malden, MA: Blackwell Publishing; 2006.

11. Mascelli MA, Zhou H, Sweet R, Getsy J, Davis HM, Graham M, et al. Molecular, biologic, and pharmacokinetic properties of monoclonal antibodies: impact of these parameters on early clinical development. J Clin Pharmacol 2007;47(5):55365.

12. Bonate PL. Recommended reading in population pharmacokinetic pharmacodynamics. AAPS J 2005;7(2):E363-73.

13. Bonate PL. Pharmacokinetic-Pharmacodynamic Modeling and Simulation. Boston, MA: Springer Science+Business Media Inc.; 2006.

14. Ette EI, Williams PJ. Population pharmacokinetics I: background, concepts, and models. Ann Pharmacother 2004;38(10):1702-6.

15. Ette EI, Williams PJ. Pharmacometrics: The Science of Quantitative Pharmacology. Hoboken, N.J.: John Wiley \& Sons; 2007.

16. Sheiner LB, Ludden TM. Population pharmacokinetics/dynamics. Annu Rev Pharmacol Toxicol 1992;32:185-209. 
17. Fang L, Holford NH, Hinkle G, Cao X, Xiao JJ, Bloomston M, et al. Population pharmacokinetics of humanized monoclonal antibody $\mathrm{HuCC} 49 \mathrm{deltaCH} 2$ and murine antibody CC49 in colorectal cancer patients. J Clin Pharmacol 2007;47(2):227-37.

18. Pescovitz MD, Knechtle S, Alexander SR, Colombani P, Nevins T, Nieforth K, et al. Safety and pharmacokinetics of daclizumab in pediatric renal transplant recipients. Pediatr Transplant 2008;12(4):447-55.

19. Meissner HC, Groothuis JR, Rodriguez WJ, Welliver RC, Hogg G, Gray PH, et al. Safety and pharmacokinetics of an intramuscular monoclonal antibody (SB 209763) against respiratory syncytial virus (RSV) in infants and young children at risk for severe RSV disease. Antimicrob Agents Chemother 1999;43(5):1183-8.

20. Beal SL, Sheiner LB, Boeckmann AJ, editors. NONMEM Users Guides. Ellicott City, MD: Icon Development Solutions; 1989-2006.

21. Ng CM, Joshi A, Dedrick RL, Garovoy MR, Bauer RJ. Pharmacokineticpharmacodynamic-efficacy analysis of efalizumab in patients with moderate to severe psoriasis. Pharm Res 2005;22(7):1088-100.

22. Ternant D, Aubourg A, Magdelaine-Beuzelin C, Degenne D, Watier H, Picon L, et al. Infliximab pharmacokinetics in inflammatory bowel disease patients. Ther Drug Monit 2008;30(4):523-9.

23. Mould DR, Baumann A, Kuhlmann J, Keating MJ, Weitman S, Hillmen P, et al. Population pharmacokinetics-pharmacodynamics of alemtuzumab (Campath) in patients with chronic lymphocytic leukaemia and its link to treatment response. $\mathrm{Br}$ J Clin Pharmacol 2007;64(3):278-91.

24. Peterson MC, Stouch BJ, Chen D, Baughman S, Holloway DL, Bekker PJ, et al. A population $\mathrm{PK} / \mathrm{PD}$ model describes the rapid, profound, and sustained suppression of urinary N-telopeptide following administration of AMG 162, a fully human monoclonal antibody against RANKL, to healthy postmenopaual women. AAPS J 2004;6(S1):[Accessed April 13, 2010] Available from: http://www.aapsj.org/abstracts/AM_2004/AAPS2004-003270.PDF.

25. Zingmark PH, Edenius C, Karlsson MO. Pharmacokinetic/pharmacodynamic models for the depletion of Vbeta5.2/5.3 T cells by the monoclonal antibody ATM-027 in patients with multiple sclerosis, as measured by FACS. Br J Clin Pharmacol 2004;58(4):378-89.

26. Mentre F, Kovarik J, Gerbeau C. Constructing a prediction interval for time to reach a threshold concentration based on a population pharmacokinetic analysis: an application to basiliximab in renal transplantation. J Pharmacokinet Biopharm 1999;27(2):213-30.

27. Kovarik JM, Nashan B, Neuhaus P, Clavien PA, Gerbeau C, Hall ML, et al. A population pharmacokinetic screen to identify demographic-clinical covariates of basiliximab in liver transplantation. Clin Pharmacol Ther 2001;69(4):201-9.

28. Lu JF, Bruno R, Eppler S, Novotny W, Lum B, Gaudreault J. Clinical pharmacokinetics of bevacizumab in patients with solid tumors. Cancer Chemother Pharmacol 2008;62(5):779-86.

29. Dirks NL, Nolting A, Kovar A, Meibohm B. Population pharmacokinetics of cetuximab in patients with squamous cell carcinoma of the head and neck. J Clin Pharmacol 2008;48(3):267-78. 
30. Mould DR, Davis CB, Minthorn EA, Kwok DC, Elliott MJ, Luggen ME, et al. A population pharmacokinetic-pharmacodynamic analysis of single doses of clenoliximab in patients with rheumatoid arthritis. Clin Pharmacol Ther 1999;66(3):246-57.

31. Yin D, Paccagnella ML, Lacy MQ, De Bono JS, Haluska P, Gualberto A, et al. Population pharmacokinetics of CP-751,871, a monoclonal antibody against IGFI receptor, in patients with multiple myeloma or solid tumors. J Clin Oncol (Meeting Abstracts) 2008;26(15_suppl):2524-. [Accessed July 01, 2009] Available from: http://www.asco.org/ASCOv2/MultiMedia/Virtual+Meeting?vmview=vm_sessio $\mathrm{n}$ presentations view\&confID $=55 \&$ sessionID $=385$.

32. Peterson MC, Jang G, Kim W, Gurrola E, Kinsey A, Dansey R. Selection of a phase 3 dose regimen for denosumab based on pharmacokinetic (PK), pharmacodynamic (PD), and safety data from multiple subcutaneous (SC) dosing regimens in breast cancer patients (pts) with bone metastases (BM). J Clin Oncol (Meeting Abstracts) 2006;24(18_suppl):3086-. [Accessed July 01, 2009] Available at: http://www.asco.org/ASCOv2/MultiMedia/Virtual+Meeting?vmview=vm_sessio $\mathrm{n}$ presentations view\&confID $=40 \&$ sessionID $=395$.

33. Sun YN, Lu JF, Joshi A, Compton P, Kwon P, Bruno RA. Population pharmacokinetics of efalizumab (humanized monoclonal anti-CD11a antibody) following long-term subcutaneous weekly dosing in psoriasis subjects. J Clin Pharmacol 2005;45(4):468-76.

34. Zhou H, Jang H, Fleischmann RM, Bouman-Thio E, Xu Z, Marini JC, et al. Pharmacokinetics and safety of golimumab, a fully human anti-TNF-alpha monoclonal antibody, in subjects with rheumatoid arthritis. J Clin Pharmacol 2007;47(3):383-96.

35. Bauer RJ, Dedrick RL, White ML, Murray MJ, Garovoy MR. Population pharmacokinetics and pharmacodynamics of the anti-CD11a antibody hu1124 in human subjects with psoriasis. J Pharmacokinet Biopharm 1999;27(4):397-420.

36. Xu Z, Seitz K, Fasanmade A, Ford J, Williamson P, Xu W, et al. Population pharmacokinetics of infliximab in patients with ankylosing spondylitis. J Clin Pharmacol 2008;48(6):681-95.

37. Fasanmade AA, Adedokun OJ, Ford J, Hernandez D, Johanns J, Hu C, et al. Population pharmacokinetic analysis of infliximab in patients with ulcerative colitis. Eur J Clin Pharmacol 2009;65(12):1211-28.

38. Dartois C, Freyer G, Michallet M, Henin E, You B, Darlavoix I, et al. Exposureeffect population model of inolimomab, a monoclonal antibody administered in first-line treatment for acute graft-versus-host disease. Clin Pharmacokinet 2007;46(5):417-32.

39. Tanswell P, Garin-Chesa P, Rettig WJ, Welt S, Divgi CR, Casper ES, et al. Population pharmacokinetics of antifibroblast activation protein monoclonal antibody F19 in cancer patients. Br J Clin Pharmacol 2001;51(2):177-80. 
40. Kuester K, Kovar A, Lupfert C, Brockhaus B, Kloft C. Population pharmacokinetic data analysis of three phase I studies of matuzumab, a humanised anti-EGFR monoclonal antibody in clinical cancer development. Br J Cancer 2008;98(5):900-6.

41. Hayashi N, Tsukamoto Y, Sallas WM, Lowe PJ. A mechanism-based binding model for the population pharmacokinetics and pharmacodynamics of omalizumab. Br J Clin Pharmacol 2007;63(5):548-61.

42. Ma P, Yang BB, Wang YM, Peterson M, Narayanan A, Sutjandra L, et al. Population pharmacokinetic analysis of panitumumab in patients with advanced solid tumors. J Clin Pharmacol 2009;49(10):1142-56.

43. Ng CM, Lum BL, Gimenez V, Kelsey S, Allison D. Rationale for fixed dosing of pertuzumab in cancer patients based on population pharmacokinetic analysis. Pharm Res 2006;23(6):1275-84.

44. Ng CM, Bruno R, Combs D, Davies B. Population pharmacokinetics of rituximab (anti-CD20 monoclonal antibody) in rheumatoid arthritis patients during a phase II clinical trial. J Clin Pharmacol 2005;45(7):792-801.

45. Kloft C, Graefe EU, Tanswell P, Scott AM, Hofheinz R, Amelsberg A, et al. Population pharmacokinetics of sibrotuzumab, a novel therapeutic monoclonal antibody, in cancer patients. Invest New Drugs 2004;22(1):39-52.

46. Bruno R, Washington CB, Lu JF, Lieberman G, Banken L, Klein P. Population pharmacokinetics of trastuzumab in patients with HER2+ metastatic breast cancer. Cancer Chemother Pharmacol 2005;56(4):361-9.

47. Zhu Y, Hu C, Lu M, Liao S, Marini JC, Yohrling J, et al. Population pharmacokinetic modeling of ustekinumab, a human monoclonal antibody targeting IL-12/23p40, in patients with moderate to severe plaque psoriasis. J Clin Pharmacol 2009;49(2):162-75.

48. Supersaxo A, Hein WR, Steffen H. Effect of molecular weight on the lymphatic absorption of water-soluble compounds following subcutaneous administration. Pharm Res 1990;7(2):167-9.

49. McLennan DN, Porter CJ, Edwards GA, Martin SW, Heatherington AC, Charman SA. Lymphatic absorption is the primary contributor to the systemic availability of epoetin Alfa following subcutaneous administration to sheep. J Pharmacol Exp Ther 2005;313(1):345-51.

50. Kagan L, Gershkovich P, Mendelman A, Amsili S, Ezov N, Hoffman A. The role of the lymphatic system in subcutaneous absorption of macromolecules in the rat model. Eur J Pharm Biopharm 2007;67(3):759-65.

51. O'Driscoll CM. Anatomy and Physiology of the Lymphatics. In: Charman WN, Stella VJ, editors. Lymphatic Transport of Drugs. Boca Raton, FL: CRC Press; 1992. p. 1-36.

52. Garg A, Balthasar JP. Physiologically-based pharmacokinetic (PBPK) model to predict IgG tissue kinetics in wild-type and FcRn-knockout mice. J Pharmacokinet Pharmacodyn 2007;34(5):687-709.

53. Tabrizi M, Bornstein GG, Suria H. Biodistribution mechanisms of therapeutic monoclonal antibodies in health and disease. AAPS J 2010;12(1):33-43.

54. Ghetie V, Ward ES. FcRn: the MHC class I-related receptor that is more than an IgG transporter. Immunol Today 1997;18(12):592-8. 
55. Ward ES, Zhou J, Ghetie V, Ober RJ. Evidence to support the cellular mechanism involved in serum IgG homeostasis in humans. Int Immunol 2003;15(2):187-95.

56. Antohe F, Radulescu L, Gafencu A, Ghetie V, Simionescu M. Expression of functionally active FcRn and the differentiated bidirectional transport of IgG in human placental endothelial cells. Hum Immunol 2001;62(2):93-105.

57. Claypool SM, Dickinson BL, Yoshida M, Lencer WI, Blumberg RS. Functional reconstitution of human FcRn in Madin-Darby canine kidney cells requires coexpressed human beta 2-microglobulin. J Biol Chem 2002;277(31):28038-50.

58. Dickinson BL, Badizadegan K, Wu Z, Ahouse JC, Zhu X, Simister NE, et al. Bidirectional FcRn-dependent IgG transport in a polarized human intestinal epithelial cell line. J Clin Invest 1999;104(7):903-11.

59. Kobayashi N, Suzuki Y, Tsuge T, Okumura K, Ra C, Tomino Y. FcRn-mediated transcytosis of immunoglobulin $\mathrm{G}$ in human renal proximal tubular epithelial cells. Am J Physiol Renal Physiol 2002;282(2):F358-65.

60. McCarthy KM, Yoong Y, Simister NE. Bidirectional transcytosis of IgG by the rat neonatal $\mathrm{Fc}$ receptor expressed in a rat kidney cell line: a system to study protein transport across epithelia. J Cell Sci 2000;113(Pt 7):1277-85.

61. Praetor A, Ellinger I, Hunziker W. Intracellular traffic of the MHC class I-like IgG Fc receptor, FcRn, expressed in epithelial MDCK cells. J Cell Sci 1999;112(Pt 14):2291-9.

62. Tuma PL, Hubbard AL. Transcytosis: crossing cellular barriers. Physiol Rev 2003;83(3):871-932.

63. Tang L, Persky AM, Hochhaus G, Meibohm B. Pharmacokinetic aspects of biotechnology products. J Pharm Sci 2004;93(9):2184-204.

64. Waldmann TA, Strober W. Metabolism of immunoglobulins. Prog Allergy 1969;13:1-110.

65. Straughn AB. Limitations of Noncompartmental Pharmacokinetic Analysis of Biotech Drugs. In: Meibohm B, editor. Pharmacokinetics and Pharmacodynamics of Biotech Drugs: Principles and Case Studies in Drug Development. Weinheim, Germany: Wiley-VCH; 2006. p. 181-8.

66. Lote CJ. Principles of Renal Physiology. 4th ed. Boston, MA: Kluwer Academic Publishers; 2000.

67. Wu CT, Davis PA, Luketic VA, Gershwin ME. A review of the physiological and immunological functions of biliary epithelial cells: targets for primary biliary cirrhosis, primary sclerosing cholangitis and drug-induced ductopenias. Clin Dev Immunol 2004;11(3-4):205-13.

68. Brambell FW. The transmission of immunity from mother to young and the catabolism of immunoglobulins. Lancet 1966;2(7473):1087-93.

69. Junghans RP. Finally! The Brambell receptor (FcRB). Mediator of transmission of immunity and protection from catabolism for IgG. Immunol Res 1997;16(1):29-57.

70. Ober RJ, Martinez C, Vaccaro C, Zhou J, Ward ES. Visualizing the site and dynamics of IgG salvage by the MHC class I-related receptor, FcRn. J Immunol 2004;172(4):2021-9.

71. Morell A, Terry WD, Waldmann TA. Metabolic properties of IgG subclasses in man. J Clin Invest 1970;49(4):673-80. 
72. Kim J, Hayton WL, Robinson JM, Anderson CL. Kinetics of FcRn-mediated recycling of IgG and albumin in human: pathophysiology and therapeutic implications using a simplified mechanism-based model. Clin Immunol 2007;122(2):146-55.

73. Daba MB. FcRn Expresion, Ligands Binding Properties and its Regulation in Human Immune Cells and Hepatocytes [thesis]. Oslo: University of Oslo; 2007.

74. Roopenian DC, Akilesh S. FcRn: the neonatal Fc receptor comes of age. Nat Rev Immunol 2007;7(9):715-25.

75. Sigal LH. Basic science for the clinician 47[corrected]: Fcgamma receptors. J Clin Rheumatol 2007;13(6):355-8.

76. Levy G. Pharmacologic target-mediated drug disposition. Clin Pharmacol Ther 1994;56(3):248-52.

77. Mager DE. Target-mediated drug disposition and dynamics. Biochem Pharmacol 2006;72(1):1-10.

78. Marathe A, Peterson MC, Mager DE. Integrated cellular bone homeostasis model for denosumab pharmacodynamics in multiple myeloma patients. J Pharmacol Exp Ther 2008;326(2):555-62.

79. Tabrizi MA, Roskos LK. Exposure-Response Relationships for Therapeutic Biologic Products. In: Meibohm B, editor. Pharmacokinetics and Pharmacodynamics of Biotech Drugs: Principles and Case Studies in Drug Development. Weinheim, Germany: Wiley-VCH; 2006. p. 295-330.

80. Tan AR, Moore DF, Hidalgo M, Doroshow JH, Poplin EA, Goodin S, et al. Pharmacokinetics of cetuximab after administration of escalating single dosing and weekly fixed dosing in patients with solid tumors. Clin Cancer Res 2006;12(21):6517-22.

81. Baselga J, Pfister D, Cooper MR, Cohen R, Burtness B, Bos M, et al. Phase I studies of anti-epidermal growth factor receptor chimeric antibody $\mathrm{C} 225$ alone and in combination with cisplatin. J Clin Oncol 2000;18(4):904-14.

82. Tokuda Y, Watanabe T, Omuro Y, Ando M, Katsumata N, Okumura A, et al. Dose escalation and pharmacokinetic study of a humanized anti-HER2 monoclonal antibody in patients with HER2/neu-overexpressing metastatic breast cancer. Br J Cancer 1999;81(8):1419-25.

83. Godfrey KR, Chapman MJ, Vajda S. Identifiability and indistinguishability of nonlinear pharmacokinetic models. J Pharmacokinet Biopharm 1994;22(3):22951.

84. Gibiansky L, Gibiansky E. Target-mediated drug disposition model: approximations, identifiability of model parameters and applications to the population pharmacokinetic-pharmacodynamic modeling of biologics. Expert Opin Drug Metab Toxicol 2009;5(7):803-12.

85. Gibiansky L, Gibiansky E, Kakkar T, Ma P. Approximations of the targetmediated drug disposition model and identifiability of model parameters. J Pharmacokinet Pharmacodyn 2008;35(5):573-91.

86. Khosla S. Minireview: the OPG/RANKL/RANK system. Endocrinology 2001;142(12):5050-5.

87. Lewiecki EM. Denosumab: an investigational drug for the management of postmenopausal osteoporosis. Biologics 2008;2(4):645-53. 
88. Cohenuram M, Saif MW. Panitumumab the first fully human monoclonal antibody: from the bench to the clinic. Anticancer Drugs 2007;18(1):7-15.

89. Kim GP, Grothey A. Targeting colorectal cancer with human anti-EGFR monoclonocal antibodies: focus on panitumumab. Biologics 2008;2(2):223-8.

90. Vanhoefer U, Tewes M, Rojo F, Dirsch O, Schleucher N, Rosen O, et al. Phase I study of the humanized antiepidermal growth factor receptor monoclonal antibody EMD72000 in patients with advanced solid tumors that express the epidermal growth factor receptor. J Clin Oncol 2004;22(1):175-84.

91. Ober RJ, Radu CG, Ghetie V, Ward ES. Differences in promiscuity for antibodyFcRn interactions across species: implications for therapeutic antibodies. Int Immunol 2001;13(12):1551-9.

92. Kovarik J, Breidenbach T, Gerbeau C, Korn A, Schmidt AG, Nashan B. Disposition and immunodynamics of basiliximab in liver allograft recipients. Clin Pharmacol Ther 1998;64(1):66-72.

93. Cartron G, Dacheux L, Salles G, Solal-Celigny P, Bardos P, Colombat P, et al. Therapeutic activity of humanized anti-CD20 monoclonal antibody and polymorphism in IgG Fc receptor FcgammaRIIIa gene. Blood 2002;99(3):754-8.

94. Weng WK, Levy R. Two immunoglobulin $\mathrm{G}$ fragment $\mathrm{C}$ receptor polymorphisms independently predict response to rituximab in patients with follicular lymphoma. J Clin Oncol 2003;21(21):3940-7.

95. Louis E, El Ghoul Z, Vermeire S, Dall'Ozzo S, Rutgeerts P, Paintaud G, et al. Association between polymorphism in IgG Fc receptor IIIa coding gene and biological response to infliximab in Crohn's disease. Aliment Pharmacol Ther 2004;19(5):511-9.

96. Musolino A, Naldi N, Bortesi B, Pezzuolo D, Capelletti M, Missale G, et al. Immunoglobulin $\mathrm{G}$ fragment $\mathrm{C}$ receptor polymorphisms and clinical efficacy of trastuzumab-based therapy in patients with HER-2/neu-positive metastatic breast cancer. J Clin Oncol 2008;26(11):1789-96.

97. Meibohm B, Laer S, Panetta JC, Barrett JS. Population pharmacokinetic studies in pediatrics: issues in design and analysis. AAPS J 2005;7(2):E475-87.

98. Wang DD, Zhang S, Zhao H, Men AY, Parivar K. Fixed dosing versus body sizebased dosing of monoclonal antibodies in adult clinical trials. J Clin Pharmacol 2009;49(9):1012-24.

99. Avastin ${ }^{\circledR}$ (bevacizumab): US prescribing information. South San Francisco, CA: Genentech, 2009.

100. Rituxan ${ }^{\circledR}$ (rituximab): US prescribing information. South San Francisco, CA: Genentech, 2008.

101. Levi M, Li J, Frey N, Kheoh T, Ren S, Woo M, et al. Characterization of the time-varying clearance of rituximab in nonhodgkin's lymphoma patients using a population pharmacokinetic analysis [abstract 9]. Paper presented at: The American Conference on Pharmacometrics: March 9-12, 2008; Tucson, AZ. [Accessed July 01, 2009] Available at: http://tucson2008.goacop.org/pdfs/Levi.pdf

102. Seitz K, Zhou H. Pharmacokinetic drug-drug interaction potentials for therapeutic monoclonal antibodies: reality check. J Clin Pharmacol 2007;47(9):1104-18. 
103. de Bono JS, Tolcher AW, Forero A, Vanhove GF, Takimoto C, Bauer RJ, et al. ING-1, a monoclonal antibody targeting Ep-CAM in patients with advanced adenocarcinomas. Clin Cancer Res 2004;10(22):7555-65.

104. Roskos LK, Kellermann SA, Foon KA. Human Antiglobulin Responses. In: Lotze MT, Thomson AW, editors. Measuring Immunity: Basic Science and Clinical Practice. San Diego, CA: Elsevier Academic Press; 2005. p. 172-86.

105. Mahmood I, Green MD. Pharmacokinetic and pharmacodynamic considerations in the development of therapeutic proteins. Clin Pharmacokinet 2005;44(4):33147.

106. Anderson CL, Chaudhury C, Kim J, Bronson CL, Wani MA, Mohanty S. Perspective-- FcRn transports albumin: relevance to immunology and medicine. Trends Immunol 2006;27(7):343-8.

107. Fracasso PM, Burris H, 3rd, Arquette MA, Govindan R, Gao F, Wright LP, et al. A phase 1 escalating single-dose and weekly fixed-dose study of cetuximab: pharmacokinetic and pharmacodynamic rationale for dosing. Clin Cancer Res 2007;13(3):986-93.

108. Erbitux ${ }^{\circledR}$ (cetuximab): US prescribing information. New York, NY: ImClone/Bristol-Myers Squibb, 2009.

109. Nolting A, Fox FE, Kovar A. Clinical Drug Development of Cetuximab, A Monoclonal Antibody. In: Meibohm B, editor. Pharmacokinetics and Pharmacodynamics of Biotech Drugs: Principles and Case Studies in Drug Development. Weinheim, Germany: Wiley-VCH; 2006. p. 353-72.

110. Bourhis J, Rivera F, Mesia R, Awada A, Geoffrois L, Borel C, et al. Phase I/II study of cetuximab in combination with cisplatin or carboplatin and fluorouracil in patients with recurrent or metastatic squamous cell carcinoma of the head and neck. J Clin Oncol 2006;24(18):2866-72.

111. Vermorken JB, Trigo J, Hitt R, Koralewski P, Diaz-Rubio E, Rolland F, et al. Open-label, uncontrolled, multicenter phase II study to evaluate the efficacy and toxicity of cetuximab as a single agent in patients with recurrent and/or metastatic squamous cell carcinoma of the head and neck who failed to respond to platinumbased therapy. J Clin Oncol 2007;25(16):2171-7.

112. Beal SL, Sheiner LB, editors. NONMEM Users Guide - Part VII: Conditional Estimation Methods. Ellicott City, MD: Icon Development Solutions; 1998.

113. Wang Y. Derivation of various NONMEM estimation methods. J Pharmacokinet Pharmacodyn 2007;34(5):575-93.

114. Lunn DJ, Thomas A, Best N, Spiegelhalter D. WinBUGS -- a Bayesian modelling framework: concepts, structure, and extensibility. Statistics and Computing 2000;10(4):325-37.

115. Best NG, Tan KK, Gilks WR, Spiegelhalter DJ. Estimation of population pharmacokinetics using the Gibbs sampler. J Pharmacokinet Biopharm 1995;23(4):407-35.

116. Duffull SB, Kirkpatrick CM, Green B, Holford NH. Analysis of population pharmacokinetic data using NONMEM and WinBUGS. J Biopharm Stat 2005;15(1):53-73. 
117. Goldstein NI, Prewett M, Zuklys K, Rockwell P, Mendelsohn J. Biological efficacy of a chimeric antibody to the epidermal growth factor receptor in a human tumor xenograft model. Clin Cancer Res 1995;1(11):1311-8.

118. Baselga J. The EGFR as a target for anticancer therapy--focus on cetuximab. Eur J Cancer 2001;37 Suppl 4:S16-22.

119. Citri A, Yarden Y. EGF-ERBB signalling: towards the systems level. Nat Rev Mol Cell Biol 2006;7(7):505-16.

120. Normanno N, De Luca A, Bianco C, Strizzi L, Mancino M, Maiello MR, et al. Epidermal growth factor receptor (EGFR) signaling in cancer. Gene 2006;366(1):2-16.

121. Hadari YR, Doody JF, Wang Y, Patel SN, Apblett RL, Loizos N, et al. The IgG1 monoclonal antibody cetuximab induces degradation of the epidermal growth factor receptor [abstract 234]. Paper presented at: ASCO 2004 Gastrointestinal Cancers Symposium; January 22-24, 2004; San Francisco, CA.

122. Sunada H, Magun BE, Mendelsohn J, MacLeod CL. Monoclonal antibody against epidermal growth factor receptor is internalized without stimulating receptor phosphorylation. Proc Natl Acad Sci U S A 1986;83(11):3825-9.

123. Robert F, Ezekiel MP, Spencer SA, Meredith RF, Bonner JA, Khazaeli MB, et al. Phase I study of anti--epidermal growth factor receptor antibody cetuximab in combination with radiation therapy in patients with advanced head and neck cancer. J Clin Oncol 2001;19(13):3234-43.

124. Luo FR, Yang Z, Dong H, Camuso A, McGlinchey K, Fager K, et al. Correlation of pharmacokinetics with the antitumor activity of Cetuximab in nude mice bearing the GEO human colon carcinoma xenograft. Cancer Chemother Pharmacol 2005;56(5):455-64.

125. Jonsson EN, Karlsson MO. Xpose--an S-PLUS based population pharmacokinetic/pharmacodynamic model building aid for NONMEM. Comput Methods Programs Biomed 1999;58(1):51-64.

126. Wilkins JJ. NONMEMory: a run management tool for NONMEM. Comput Methods Programs Biomed 2005;78(3):259-67.

127. Meibohm B, Evans WE. Clinical Pharmacodynamics and Pharmacokinetics. In: Helms RA, Quan DJ, Herfindal ET, Gourley DR, editors. Textbook of Therapeutics: Drug and Disease Management. 8th ed. Philadelphia: Lippincott Williams \& Wilkins; 2006. p. 1-30.

128. Bonate PL. The effect of collinearity on parameter estimates in nonlinear mixed effect models. Pharm Res 1999;16(5):709-17.

129. Maitre PO, Buhrer M, Thomson D, Stanski DR. A three-step approach combining Bayesian regression and NONMEM population analysis: application to midazolam. J Pharmacokinet Biopharm 1991;19(4):377-84.

130. Mandema JW, Verotta D, Sheiner LB. Building population pharmacokinetic-pharmacodynamic models. I. Models for covariate effects. J Pharmacokinet Biopharm 1992;20(5):511-28.

131. Rowinsky EK, Schwartz GH, Gollob JA, Thompson JA, Vogelzang NJ, Figlin R, et al. Safety, pharmacokinetics, and activity of ABX-EGF, a fully human antiepidermal growth factor receptor monoclonal antibody in patients with metastatic renal cell cancer. J Clin Oncol 2004;22(15):3003-15. 
132. Flessner MF, Lofthouse J, Zakaria el R. In vivo diffusion of immunoglobulin G in muscle: effects of binding, solute exclusion, and lymphatic removal. Am J Physiol 1997;273(6 Pt 2):H2783-93.

133. Shin DM, Donato NJ, Perez-Soler R, Shin HJ, Wu JY, Zhang P, et al. Epidermal growth factor receptor-targeted therapy with $\mathrm{C} 225$ and cisplatin in patients with head and neck cancer. Clin Cancer Res 2001;7(5):1204-13.

134. Dreux AC, Lamb DJ, Modjtahedi H, Ferns GA. The epidermal growth factor receptors and their family of ligands: their putative role in atherogenesis. Atherosclerosis 2006;186(1):38-53.

135. Feistritzer C, Mosheimer BA, Sturn DH, Riewald M, Patsch JR, Wiedermann CJ. Endothelial protein $\mathrm{C}$ receptor-dependent inhibition of migration of human lymphocytes by protein $\mathrm{C}$ involves epidermal growth factor receptor. J Immunol 2006;176(2):1019-25.

136. Lewkowicz P, Tchorzewski H, Dytnerska K, Banasik M, Lewkowicz N. Epidermal growth factor enhances TNF-alpha-induced priming of human neutrophils. Immunol Lett 2005;96(2):203-10.

137. Meibohm B, Braeckman RA. Pharmacokinetics and Pharmacodynamics of Peptide and Protein Drugs. In: Crommelin DJA, Sindelar RD, Meibohm B, editors. Pharmaceutical Biotechnology. 3rd ed. New York, NY: Informa Healthcare; 2007. p. 95-123.

138. DuBois D, DuBois EF. A formula to estimate the approximate surface area if height and weight be known. Arch Intern Med 1916;17:863-71.

139. Cooper MR, Cohen R, Anderson V, Falcey J, Waksal H. Pharmacokinetics of a chimerized monoclonal antibody (C225) against the epidermal growth factor receptor (EGFr) [Abstr 834]. Paper presented at: 1998 ASCO Annual Meeting; May 17-19, 1998; Los Angeles, CA.

140. Fox FE, Mauro D, Bai S, Raymond R, Farrell C, Pentikis H, et al. A population pharmacokinetic (PPK) analysis of the anti-EGFr specific IgG1 monoclonal antibody cetuximab [abstract290]. Paper presented at: ASCO 2004 Gastrointestinal Cancers Symposium: January 22-24, 2004; San Francisco, CA.

141. Delbaldo C, Pierga JY, Dieras V, Faivre S, Laurence V, Vedovato JC, et al. Pharmacokinetic profile of cetuximab (Erbitux) alone and in combination with irinotecan in patients with advanced EGFR-positive adenocarcinoma. Eur J Cancer 2005;41(12):1739-45.

142. Bennett JE, Wakefield JC. A comparison of a Bayesian population method with two methods as implemented in commercially available software. J Pharmacokinet Biopharm 1996;24(4):403-32.

143. Bustad A, Terziivanov D, Leary R, Port R, Schumitzky A, Jelliffe R. Parametric and nonparametric population methods: their comparative performance in analysing a clinical dataset and two Monte Carlo simulation studies. Clin Pharmacokinet 2006;45(4):365-83.

144. Dartois C, Lemenuel-Diot A, Laveille C, Tranchand B, Tod M, Girard P. Evaluation of uncertainty parameters estimated by different population PK software and methods. J Pharmacokinet Pharmacodyn 2007;34(3):289-311. 
145. Girard P, Mentre F. A comparison of estimation methods in nonlinear mixed effects models using a blind analysis [abstract 834]. Paper presented at: Population approach group in europe (PAGE) 2005 annual meeting; June 16-17, 2005; Pamplona, Spain.

146. Roe DJ. Comparison of population pharmacokinetic modeling methods using simulated data: results from the Population Modeling Workgroup. Stat Med 1997;16(11):1241-57; discussion 1257-62.

147. White DB, Walawander CA, Tung Y, Grasela TH. An evaluation of point and interval estimates in population pharmacokinetics using NONMEM analysis. J Pharmacokinet Biopharm 1991;19(1):87-112.

148. Hashimoto Y, Koue T, Otsuki Y, Yasuhara M, Hori R, Inui K. Simulation for population analysis of Michaelis-Menten elimination kinetics. J Pharmacokinet Biopharm 1995;23(2):205-16.

149. Sheiner LB, Beal SL. Evaluation of methods for estimating population pharmacokinetics parameters. I. Michaelis-Menten model: routine clinical pharmacokinetic data. J Pharmacokinet Biopharm 1980;8(6):553-71.

150. Bauer RJ, Guzy S, Ng C. A survey of population analysis methods and software for complex pharmacokinetic and pharmacodynamic models with examples. AAPS J 2007;9(1):E60-83.

151. Dartois C, Brendel K, Comets E, Laffont CM, Laveille C, Tranchand B, et al. Overview of model-building strategies in population PK/PD analyses: 2002-2004 literature survey. Br J Clin Pharmacol 2007;64(5):603-12.

152. Lunn DJ, Best N, Thomas A, Wakefield J, Spiegelhalter D. Bayesian analysis of population PK/PD models: general concepts and software. J Pharmacokinet Pharmacodyn 2002;29(3):271-307.

153. R Development Core Team (2009). R: A language and environment for statistical computing. R Foundation for Statistical Computing, Vienna, Austria. [Accessed May 01, 2008] Available at: http://www.r-project.org/.

154. Knebel W, Bergsma T, Fisher J, Georgalis G, Gibiansky L, Gillespie B, et al. Facilitating the pharmacometrics work-flow with the MItools R package [abstract 30]. Paper presented at: 2008 American Conference on Pharmacometrics; March 9-12, 2008; Tucson, AZ [Accessed July 01, 2009] Available at: http://tucson2008.go-acop.org/pdfs/30_Knebel.pdf.

155. Sparreboom A, Wolff AC, Mathijssen RH, Chatelut E, Rowinsky EK, Verweij J, et al. Evaluation of alternate size descriptors for dose calculation of anticancer drugs in the obese. J Clin Oncol 2007;25(30):4707-13.

156. Foracchia M, Hooker A, Vicini P, Ruggeri A. POPED, a software for optimal experiment design in population kinetics. Comput Methods Programs Biomed 2004;74(1):29-46.

157. Gillespie WR, editor. Prototype PKPD Model Library for WinBUGS User Manual: Version 1.1. Augusta, ME: Metrum Institute; 2009.

158. Lunn DJ. WinBUGS Development Interface (WBDev). ISBA Bulletin 2003;10(3).

159. Plummer M, Best N, Cowles K, Vines K. coda: Output analysis and diagnostics for MCMC. R package version 0.13-4. 2009. 
160. Sturtz S, Ligges U, Gelman A. R2WinBUGS: A package for running WinBUGS from R. Journal of Statistical Software 2005;12(3):1-16.

161. Gelman A. Prior distributions for variance parameters in hierarchical models (Comment on Article by Browne and Draper). Bayesian Analysis 2006;1(3):51534.

162. Brooks SP, Gelman A. General methods for monitoring convergence of iterative simulations. J Comput Graph Stat 1998;7(4):434-55.

163. Jonsson EN, Wade JR, Karlsson MO. Nonlinearity detection: advantages of nonlinear mixed-effects modeling. AAPS PharmSci 2000;2(3):E32.

164. Bauer R, Ludden T. Improvements and new estimation methods in NONMEM 7 for PK/PD population analysis [abstract 1516]. Paper presented at: Population Approach Group Europe 18th Annual Meeting: June 23-26, 2009; St. Petersburg, Russia. 
APPENDIX A:

NONMEM CONTROL STREAM AND OUTPUT FOR THE FINAL CETUXIMAB POPULATION PK MODEL 
A-1. NONMEM control stream for the final cetuximab population pharmacokinetic model.

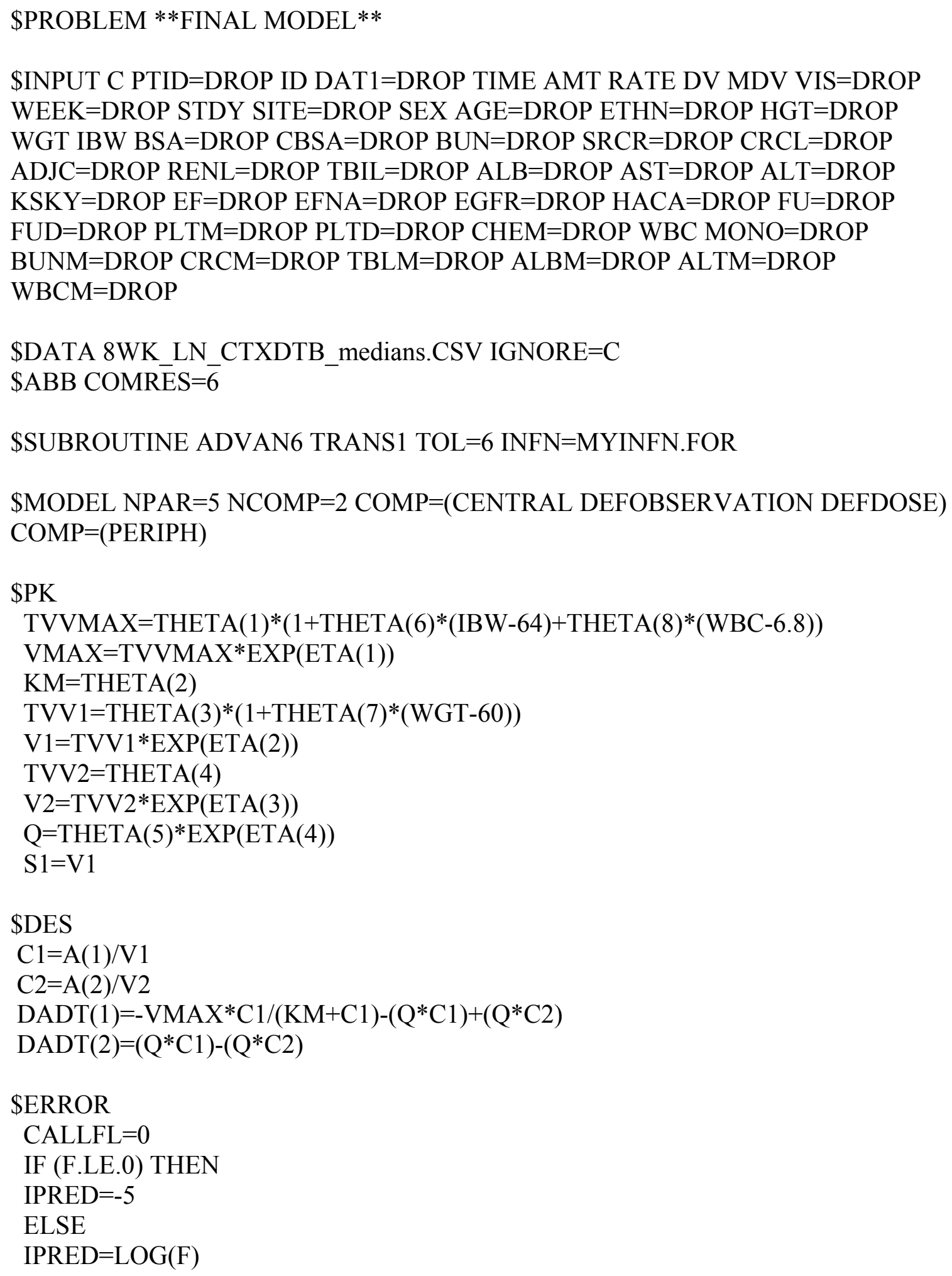




\author{
ENDIF \\ IRES=DV-IPRED \\ IWRES=IRES/IPRED \\ IF (STDY.EQ.8) THEN \\ $\mathrm{Y}=\mathrm{IPRED}+\mathrm{ERR}(1)$ \\ ELSE \\ $\mathrm{Y}=\mathrm{IPRED}+\mathrm{ERR}(2)$ \\ ENDIF
}

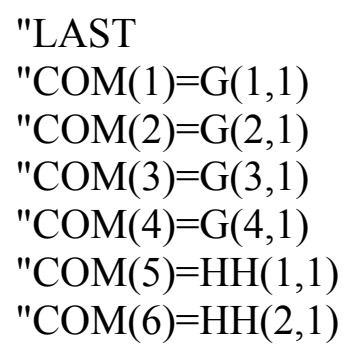

0.030

0.050

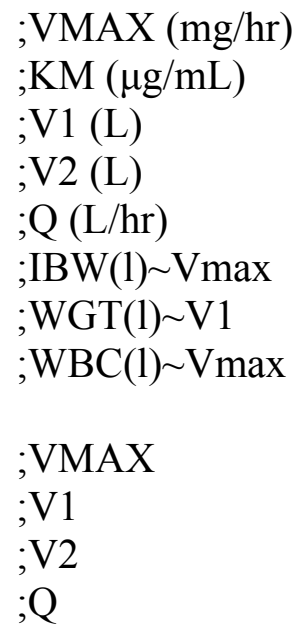

\$ESTIMATION NOABORT MAXEVAL=9999 PRINT=5 METHOD=1 \$COVARIANCE PRINT $=\mathrm{E}$

\$TABLE ID TIME IPRED IWRES NOPRINT ONEHEADER FILE=sdtab1077 \$TABLE ID VMAX KM V1 V2 Q NOPRINT ONEHEADER FILE=patab1077 \$TABLE ID IBW WGT WBC NOPRINT ONEHEADER FILE $=\operatorname{cotab} 1077$ \$TABLE ID SEX NOPRINT ONEHEADER FILE $=$ catab 1077

$\$$ TABLE ID $\operatorname{COM}(1)=\mathrm{G} 11 \operatorname{COM}(2)=\mathrm{G} 21 \mathrm{COM}(3)=\mathrm{G} 31 \mathrm{COM}(4)=\mathrm{G} 41 \operatorname{COM}(5)=\mathrm{H} 11$ $\operatorname{COM}(6)=\mathrm{H} 21$ IPRED MDV NOPRINT ONEHEADER FILE=cwtab1077 
A-2. NONMEM run output for the final cetuximab population pharmacokinetic model.

1NONLINEAR MIXED EFFECTS MODEL PROGRAM (NONMEM)

DOUBLE PRECISION NONMEM VERSION V LEVEL 1.1

DEVELOPED AND PROGRAMMED BY STUART BEAL AND LEWIS SHEINER

$\begin{array}{lr}\text { PROBLEM NO.: } 1 & \\ \text { **FINAL MODEL** } & \text { NO } \\ \text { 0DATA CHECKOUT RUN: } & 2 \\ \text { DATA SET LOCATED ON UNIT NO.: } & \text { NO } \\ \text { THIS UNIT TO BE REWOUND: } & \text { NO. OF DATA RECS IN DATA SET: } \\ \text { NO. OF DATA ITEMS IN DATA SET: } & 13 \\ \text { ID DATA ITEM IS DATA ITEM NO.: } & 2 \\ \text { DEP VARIABLE IS DATA ITEM NO.: } & 6 \\ \text { MDV DATA ITEM IS DATA ITEM NO.: } & 7 \\ \text { OINDICES PASSED TO SUBROUTINE PRED: }\end{array}$

$\begin{array}{llllllllll}13 & 3 & 4 & 5 & 0 & 0 & 0 & 0 & 0\end{array}$

00

OLABELS FOR DATA ITEMS:

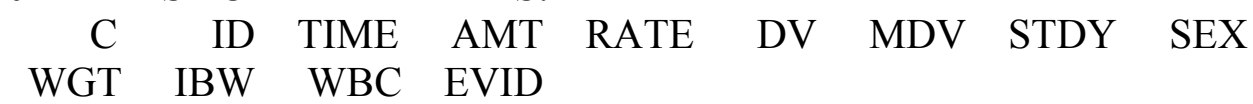
0(NONBLANK) LABELS FOR PRED-DEFINED ITEMS:

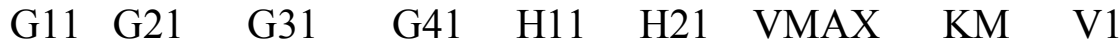

V2 Q IPRE IWRE

0FORMAT FOR DATA:

(E2.0,E4.0,E8.0,2E7.0,E12.0,E2.0,E3.0,E2.0,E6.0,E7.0,E5.0,1F2.0)

TOT. NO. OF OBS RECS: 912

TOT. NO. OF INDIVIDUALS: 143

OLENGTH OF THETA: 8

0OMEGA HAS SIMPLE DIAGONAL FORM WITH DIMENSION: 4 OSIGMA HAS SIMPLE DIAGONAL FORM WITH DIMENSION: 2 OINITIAL ESTIMATE OF THETA:

LOWER BOUND INITIAL EST UPPER BOUND

$\begin{array}{lll}0.1500 \mathrm{E}+01 & 0.4220 \mathrm{E}+01 & 0.7500 \mathrm{E}+01\end{array}$

$0.3500 \mathrm{E}+02 \quad 0.7160 \mathrm{E}+02 \quad 0.1500 \mathrm{E}+03$

$\begin{array}{lll}0.1300 \mathrm{E}+01 & 0.2720 \mathrm{E}+01 & 0.5000 \mathrm{E}+01\end{array}$

$0.1100 \mathrm{E}+01 \quad 0.2430 \mathrm{E}+01 \quad 0.5000 \mathrm{E}+01$

$0.1000 \mathrm{E}-02 \quad 0.9500 \mathrm{E}-01 \quad 0.1500 \mathrm{E}+00$

$0.1000 \mathrm{E}-02 \quad 0.1150 \mathrm{E}-01 \quad 0.5000 \mathrm{E}-01$

$0.1000 \mathrm{E}-02 \quad 0.8000 \mathrm{E}-02 \quad 0.5000 \mathrm{E}-01$

$0.1000 \mathrm{E}-02 \quad 0.2400 \mathrm{E}-01 \quad 0.7500 \mathrm{E}-01$ 


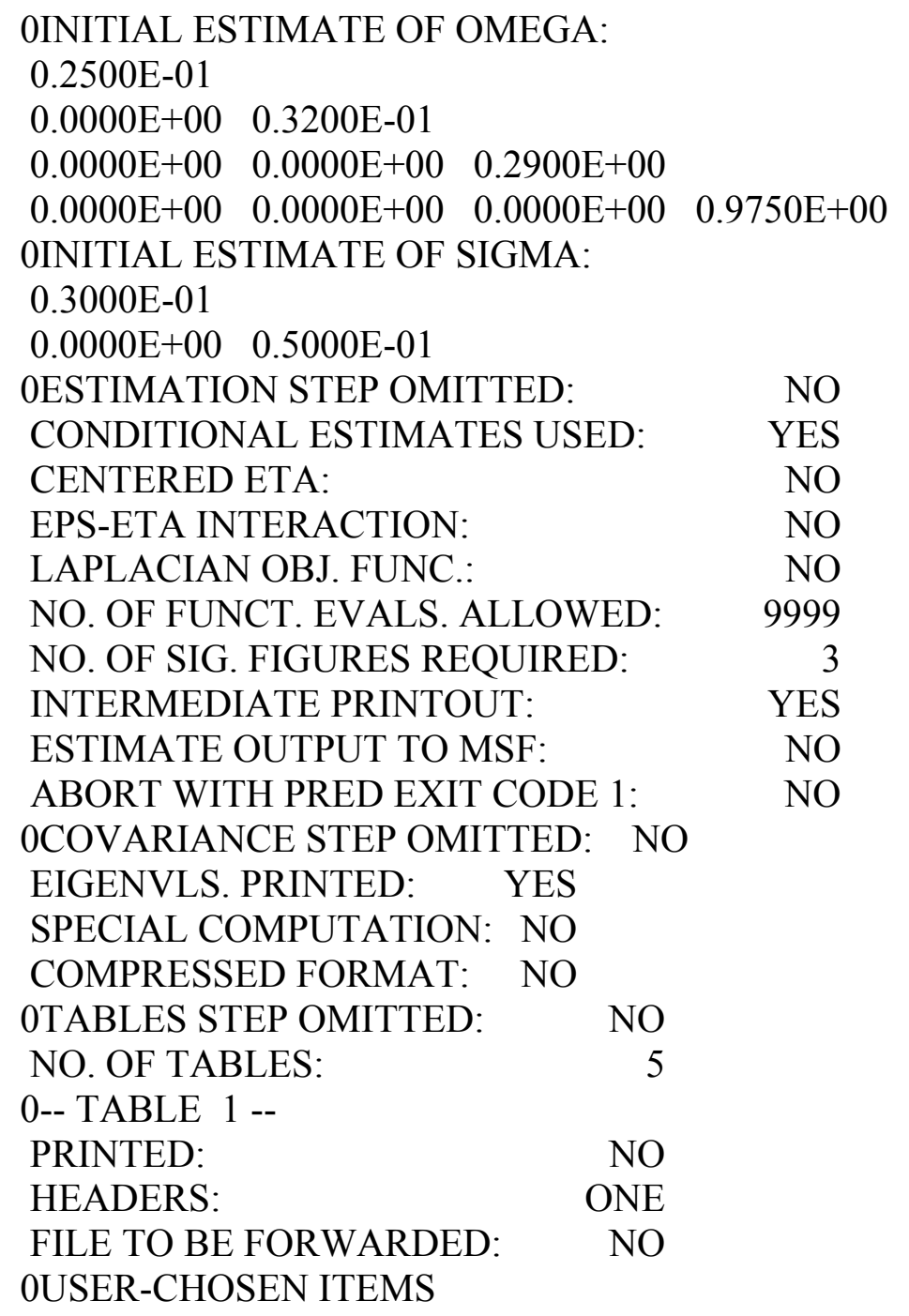

IN THE ORDER THEY WILL APPEAR IN THE TABLE:

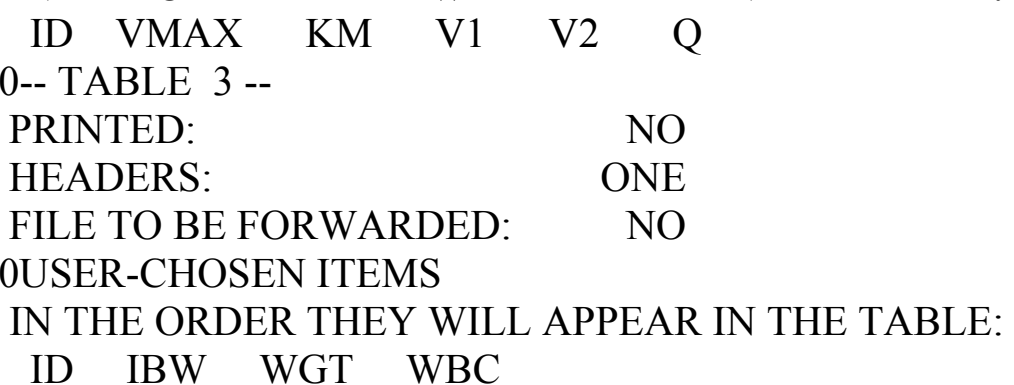


0-- TABLE 4 --

PRINTED: NO

HEADERS: $\quad$ ONE

FILE TO BE FORWARDED: NO

OUSER-CHOSEN ITEMS

IN THE ORDER THEY WILL APPEAR IN THE TABLE:

ID SEX

0-- TABLE 5 --

PRINTED: $\quad$ NO

HEADERS: $\quad$ ONE

FILE TO BE FORWARDED: $\quad$ NO

OUSER-CHOSEN ITEMS

IN THE ORDER THEY WILL APPEAR IN THE TABLE:

ID G11 G21 G31 G41 H11 H21 IPRE MDV

1DOUBLE PRECISION PREDPP VERSION IV LEVEL 1.1

GENERAL NONLINEAR KINETICS MODEL (ADVAN6)

OMODEL SUBROUTINE USER-SUPPLIED - ID NO. 9999

OMAXIMUM NO. OF BASIC PK PARAMETERS: 5

OCOMPARTMENT ATTRIBUTES

COMPT. NO. FUNCTION INITIAL ON/OFF DOSE DEFAULT DEFAULT

STATUS ALLOWED ALLOWED FOR DOSE FOR OBS.

$\begin{array}{lllllll}1 & \text { CENTRAL } & \text { ON } & \text { YES } & \text { YES } & \text { YES } & \text { YES } \\ 2 & \text { PERIPH } & \text { ON } & \text { YES } & \text { YES } & \text { NO } & \text { NO } \\ 3 & \text { OUTPUT } & \text { OFF } & \text { YES } & \text { NO } & \text { NO } & \text { NO }\end{array}$

ONRD VALUE FROM SUBROUTINE TOL: 6

1

ADDITIONAL PK PARAMETERS - ASSIGNMENT OF ROWS IN GG

COMPT. NO.

INDICES

SCALE BIOAVAIL. ZERO-ORDER ZERO-ORDER ABSORB

FRACTION RATE DURATION LAG

$\begin{array}{llllll}1 & 6 & * & * & * & * \\ 2 & * & * & * & * & * \\ 3 & * & - & - & - & -\end{array}$

- PARAMETER IS NOT ALLOWED FOR THIS MODEL

* PARAMETER IS NOT SUPPLIED BY PK SUBROUTINE;

WILL DEFAULT TO ONE IF APPLICABLE

ODATA ITEM INDICES USED BY PRED ARE:

EVENT ID DATA ITEM IS DATA ITEM NO.: 13

TIME DATA ITEM IS DATA ITEM NO.: 3

DOSE AMOUNT DATA ITEM IS DATA ITEM NO.: 4

DOSE RATE DATA ITEM IS DATA ITEM NO.: 5

OPK SUBROUTINE CALLED WITH EVERY EVENT RECORD.

PK SUBROUTINE NOT CALLED AT NONEVENT (ADDITIONAL OR LAGGED) DOSE TIMES. 
ODURING SIMULATION, ERROR SUBROUTINE CALLED WITH EVERY EVENT RECORD.

OTHERWISE, ERROR SUBROUTINE CALLED ONLY WITH OBSERVATION

EVENTS.

ODES SUBROUTINE USES COMPACT STORAGE MODE

1

MONITORING OF SEARCH:

OITERATION NO.: 0 OBJECTIVE VALUE: -0.1720E+04 NO. OF FUNC. EVALS.:12 CUMULATIVE NO. OF FUNC. EVALS.: 12

PARAMETER: $0.1000 \mathrm{E}+00 \quad 0.1000 \mathrm{E}+00 \quad 0.1000 \mathrm{E}+00 \quad 0.1000 \mathrm{E}+00 \quad 0.1000 \mathrm{E}+00$

$\begin{array}{lllllll}0.1000 \mathrm{E}+00 & 0.1000 \mathrm{E}+00 & 0.1000 \mathrm{E}+00 & 0.1000 \mathrm{E}+00 & 0.1000 \mathrm{E}+00\end{array}$

$\begin{array}{llll}0.1000 \mathrm{E}+00 & 0.1000 \mathrm{E}+00 & 0.1000 \mathrm{E}+00 & 0.1000 \mathrm{E}+00\end{array}$

GRADIENT: $-0.1987 \mathrm{E}+04 \quad 0.7020 \mathrm{E}+03-0.1821 \mathrm{E}+04-0.7369 \mathrm{E}+02-0.1583 \mathrm{E}+03$

$\begin{array}{llll}0.1211 \mathrm{E}+03 & -0.3783 \mathrm{E}+02 \quad 0.6401 \mathrm{E}+02 \quad 0.1225 \mathrm{E}+03 \quad 0.6147 \mathrm{E}+02\end{array}$

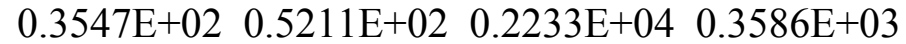

OITERATION NO.: 5 OBJECTIVE VALUE: -0.1734E+04 NO. OF FUNC. EVALS.:16 CUMULATIVE NO. OF FUNC. EVALS.: 95

$\begin{array}{llllll}\text { PARAMETER: } & 0.1022 \mathrm{E}+00 & 0.1018 \mathrm{E}+00 & 0.1111 \mathrm{E}+00 & 0.1004 \mathrm{E}+00 & 0.1054 \mathrm{E}+00\end{array}$

$0.9592 \mathrm{E}-01 \quad 0.1017 \mathrm{E}+00 \quad 0.9341 \mathrm{E}-01 \quad 0.9685 \mathrm{E}-01 \quad 0.1023 \mathrm{E}+00$

$0.9986 \mathrm{E}-01 \quad 0.9828 \mathrm{E}-01 \quad 0.7662 \mathrm{E}-01 \quad 0.9383 \mathrm{E}-01$

GRADIENT: $-0.2774 \mathrm{E}+03 \quad 0.1573 \mathrm{E}+03 \quad 0.2254 \mathrm{E}+04 \quad 0.5423 \mathrm{E}+02 \quad 0.1088 \mathrm{E}+03-$

$0.1481 \mathrm{E}+02-0.5559 \mathrm{E}+01 \quad 0.2991 \mathrm{E}+02-0.5791 \mathrm{E}+02-0.2172 \mathrm{E}+03$

$-0.5601 \mathrm{E}+02-0.2452 \mathrm{E}+02-0.2079 \mathrm{E}+04-0.1212 \mathrm{E}+03$

OITERATION NO.:10 OBJECTIVE VALUE: -0.1734E+04 NO. OF FUNC. EVALS.:28

CUMULATIVE NO. OF FUNC. EVALS.: 193

PARAMETER: $0.1023 \mathrm{E}+00 \quad 0.1018 \mathrm{E}+00 \quad 0.1107 \mathrm{E}+00 \quad 0.1021 \mathrm{E}+00 \quad 0.1135 \mathrm{E}+00$

$0.9619 \mathrm{E}-01 \quad 0.1030 \mathrm{E}+00 \quad 0.9554 \mathrm{E}-01 \quad 0.9668 \mathrm{E}-01 \quad 0.1037 \mathrm{E}+00$

$0.1026 \mathrm{E}+00 \quad 0.9905 \mathrm{E}-01 \quad 0.7667 \mathrm{E}-01 \quad 0.9333 \mathrm{E}-01$

GRADIENT: $-0.3024 \mathrm{E}+03 \quad 0.1401 \mathrm{E}+03 \quad 0.2190 \mathrm{E}+04 \quad 0.6069 \mathrm{E}+02 \quad 0.1494 \mathrm{E}+03-$

$0.2381 \mathrm{E}+01 \quad 0.9320 \mathrm{E}+01 \quad 0.9397 \mathrm{E}+02-0.5406 \mathrm{E}+02-0.1898 \mathrm{E}+03$

$-0.4180 \mathrm{E}+02-0.3083 \mathrm{E}+02-0.2064 \mathrm{E}+04-0.1451 \mathrm{E}+03$

OITERATION NO.: 15 OBJECTIVE VALUE: -0.1735E+04 NO. OF FUNC. EVALS.:24

CUMULATIVE NO. OF FUNC. EVALS.: 329

PARAMETER: $0.1107 \mathrm{E}+00 \quad 0.1230 \mathrm{E}+00 \quad 0.1108 \mathrm{E}+00 \quad 0.9981 \mathrm{E}-01 \quad 0.1108 \mathrm{E}+00$

$0.9788 \mathrm{E}-01 \quad 0.9636 \mathrm{E}-01 \quad 0.9667 \mathrm{E}-01 \quad 0.1018 \mathrm{E}+00 \quad 0.1054 \mathrm{E}+00$

$0.1069 \mathrm{E}+00 \quad 0.1026 \mathrm{E}+00 \quad 0.7697 \mathrm{E}-01 \quad 0.9319 \mathrm{E}-01$

GRADIENT: $-0.3194 \mathrm{E}+03 \quad 0.1718 \mathrm{E}+03 \quad 0.2123 \mathrm{E}+04 \quad 0.4061 \mathrm{E}+02 \quad 0.1482 \mathrm{E}+03$

$0.1371 \mathrm{E}+02-0.6102 \mathrm{E}+02 \quad 0.9505 \mathrm{E}+02-0.3377 \mathrm{E}+02-0.1517 \mathrm{E}+03$

$-0.2690 \mathrm{E}+02-0.1610 \mathrm{E}+02-0.1952 \mathrm{E}+04-0.1347 \mathrm{E}+03$

OITERATION NO.: 20 OBJECTIVE VALUE: -0.1749E+04 NO. OF FUNC. EVALS.:23

CUMULATIVE NO. OF FUNC. EVALS.: 445

PARAMETER: $0.1035 \mathrm{E}+00 \quad 0.1037 \mathrm{E}+00 \quad 0.1044 \mathrm{E}+00 \quad 0.1001 \mathrm{E}+00 \quad 0.1064 \mathrm{E}+00$

$0.9633 \mathrm{E}-01 \quad 0.1022 \mathrm{E}+00 \quad 0.9394 \mathrm{E}-01 \quad 0.9712 \mathrm{E}-01 \quad 0.1039 \mathrm{E}+00$

$0.1045 \mathrm{E}+00 \quad 0.9996 \mathrm{E}-01 \quad 0.8401 \mathrm{E}-01 \quad 0.9484 \mathrm{E}-01$ 
GRADIENT: $-0.5994 \mathrm{E}+01 \quad 0.8684 \mathrm{E}+00-0.1413 \mathrm{E}+02 \quad 0.7832 \mathrm{E}+00-0.1302 \mathrm{E}+01-$

$0.1207 \mathrm{E}+01-0.7490 \mathrm{E}+00-0.9671 \mathrm{E}-01 \quad 0.5682 \mathrm{E}+00-0.2083 \mathrm{E}+01$

$-0.2933 \mathrm{E}+00 \quad 0.1312 \mathrm{E}+02 \quad 0.4625 \mathrm{E}+01-0.1651 \mathrm{E}+01$

OITERATION NO.:25 OBJECTIVE VALUE: -0.1749E+04 NO. OF FUNC. EVALS.:23

CUMULATIVE NO. OF FUNC. EVALS.: 561

PARAMETER: $0.1035 \mathrm{E}+00 \quad 0.1038 \mathrm{E}+00 \quad 0.1045 \mathrm{E}+00 \quad 0.1002 \mathrm{E}+00 \quad 0.1063 \mathrm{E}+00$

$\begin{array}{lllll}0.9638 \mathrm{E}-01 & 0.1023 \mathrm{E}+00 & 0.9393 \mathrm{E}-01 & 0.9711 \mathrm{E}-01 \quad 0.1040 \mathrm{E}+00\end{array}$

$0.1046 \mathrm{E}+00 \quad 0.9842 \mathrm{E}-01 \quad 0.8402 \mathrm{E}-01 \quad 0.9488 \mathrm{E}-01$

GRADIENT: $-0.1407 \mathrm{E}+00 \quad 0.2594 \mathrm{E}+00-0.1476 \mathrm{E}+00 \quad 0.5952 \mathrm{E}-01 \quad 0.1299 \mathrm{E}+00$

$0.9197 \mathrm{E}-01 \quad 0.1468 \mathrm{E}+00-0.4645 \mathrm{E}-01 \quad 0.8486 \mathrm{E}-01-0.3774 \mathrm{E}-01$

$-0.2707 \mathrm{E}-01 \quad 0.9374 \mathrm{E}-01 \quad 0.6367 \mathrm{E}-02-0.1270 \mathrm{E}+00$

OITERATION NO.: 26 OBJECTIVE VALUE: -0.1749E+04 NO. OF FUNC. EVALS.: 0 CUMULATIVE NO. OF FUNC. EVALS.: 561

$\begin{array}{llllll}\text { PARAMETER: } & 0.1035 \mathrm{E}+00 & 0.1038 \mathrm{E}+00 & 0.1045 \mathrm{E}+00 & 0.1002 \mathrm{E}+00 & 0.1063 \mathrm{E}+00\end{array}$

$0.9638 \mathrm{E}-01 \quad 0.1023 \mathrm{E}+00 \quad 0.9393 \mathrm{E}-01 \quad 0.9711 \mathrm{E}-01 \quad 0.1040 \mathrm{E}+00$

$0.1046 \mathrm{E}+00 \quad 0.9842 \mathrm{E}-01 \quad 0.8402 \mathrm{E}-01 \quad 0.9488 \mathrm{E}-01$

GRADIENT: $-0.1407 \mathrm{E}+00 \quad 0.2594 \mathrm{E}+00-0.1476 \mathrm{E}+00 \quad 0.5952 \mathrm{E}-01 \quad 0.1299 \mathrm{E}+00$

$0.9197 \mathrm{E}-01 \quad 0.1468 \mathrm{E}+00-0.4645 \mathrm{E}-01 \quad 0.8486 \mathrm{E}-01-0.3774 \mathrm{E}-01$

$-0.2707 \mathrm{E}-01 \quad 0.9374 \mathrm{E}-01 \quad 0.6367 \mathrm{E}-02-0.1270 \mathrm{E}+00$

OMINIMIZATION SUCCESSFUL

NO. OF FUNCTION EVALUATIONS USED: 561

NO. OF SIG. DIGITS IN FINAL EST.: $\quad 3.4$

ETABAR IS THE ARITHMETIC MEAN OF THE ETA-ESTIMATES, AND THE P-VALUE IS GIVEN FOR THE NULL HYPOTHESIS THAT THE TRUE MEAN IS 0.

ETABAR: $0.13 \mathrm{E}-02 \quad-0.11 \mathrm{E}-01 \quad-0.29 \mathrm{E}-01 \quad-0.95 \mathrm{E}-02$

$\begin{array}{llll}\text { P VAL.: } \quad 0.90 \mathrm{E}+00 & 0.34 \mathrm{E}+00 & 0.22 \mathrm{E}+00 \quad 0.82 \mathrm{E}+00\end{array}$

OR MATRIX ALGORITHMICALLY NON-POSITIVE-SEMIDEFINITE

BUT NONSINGULAR

OCOVARIANCE STEP ABORTED

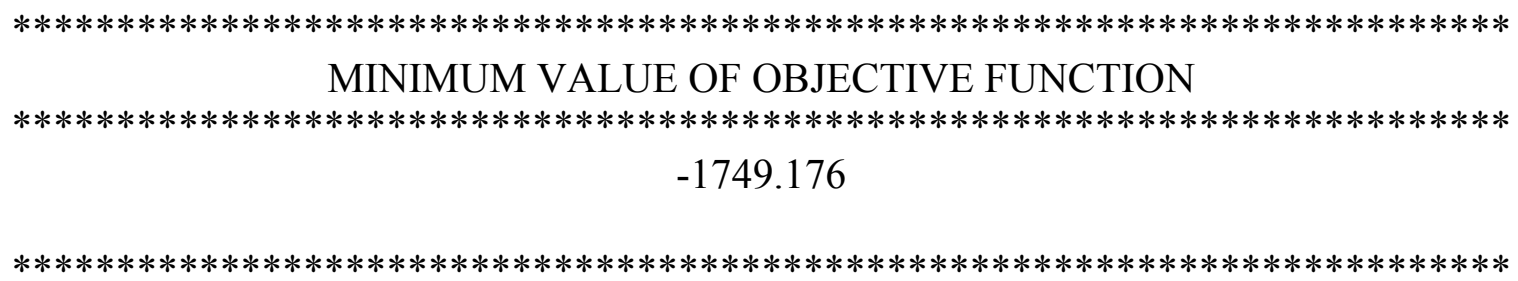




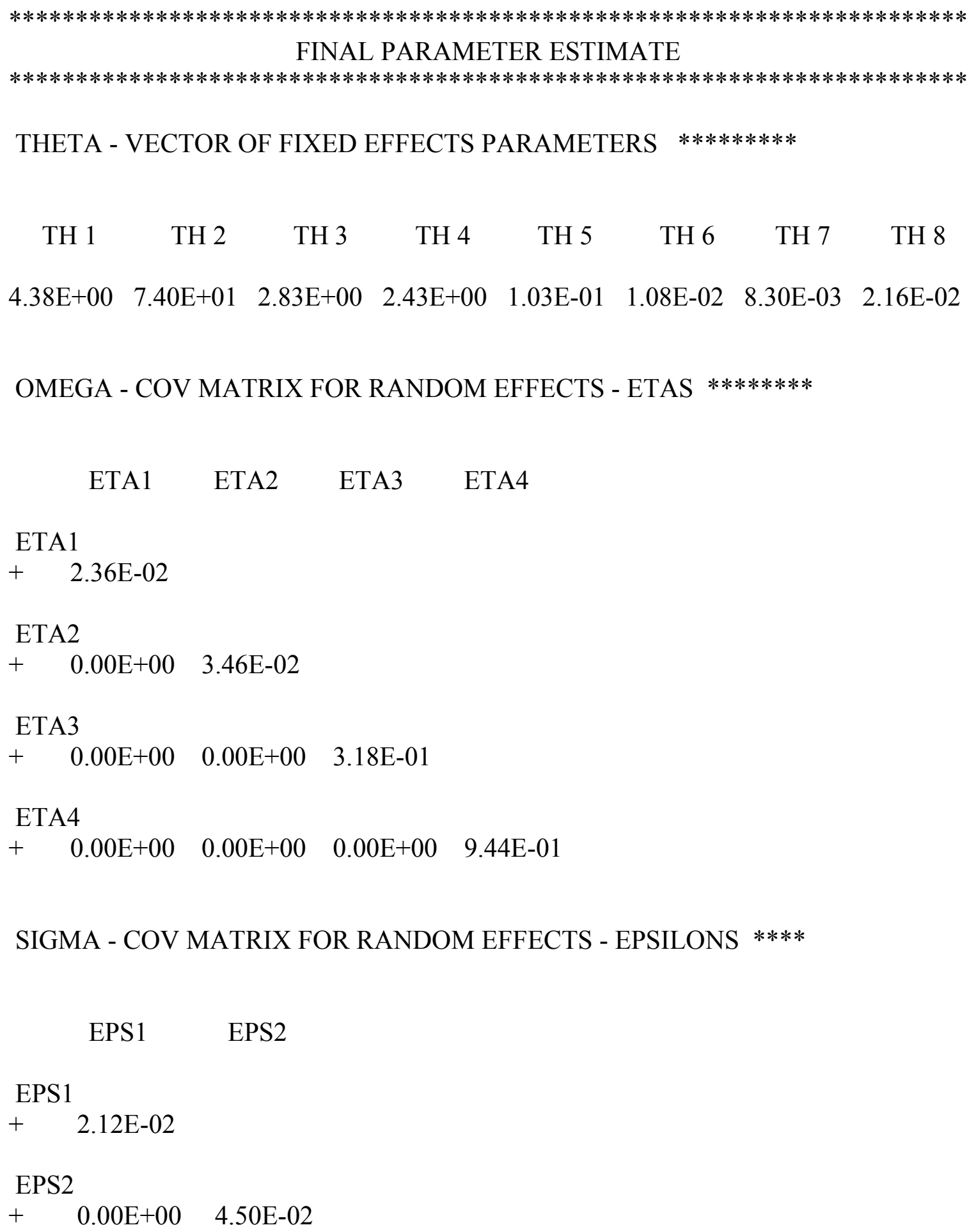


APPENDIX B:

EXAMPLE COMPUTER CODES USED FOR THE SIMULATION AND

ESTIMATION STEPS IN CHAPTER 4 
B-1. R script used to sample parameter values from their specified simulation prior (uncertainty) distributions.

\#Set seed for reproducibility

set.seed(48215)

$\operatorname{rnorm}(5)$

\#[1] $0.5228567-2.2998926 \quad 0.6365216-0.5349108 \quad 0.6051626$

\#Simulate Vmax $(\mathrm{mg} / \mathrm{hr})$ thetas from a lognormal distribution

vmax.theta.vct<-rlnorm $(\mathrm{n}=1000$, mean $\log =\log (0.75), \mathrm{sdlog}=0.1)$

head(vmax.theta.vct)

\#Convert vector of simulated Vmax thetas to dataframe; assign column name

vmax.theta<-data.frame(vmax.theta.vet)

$\operatorname{dim}($ vmax.theta)

names(vmax.theta)<-"VMAX_TH"

head(vmax.theta)

$\# * * * \# * * * \# * * * \# * * * \# * * * \# * * * \# * * * \#$

\#Simulate $\mathrm{Km}(\mathrm{mg} / \mathrm{L})$ thetas from a lognormal distribution

km.theta.vct<-rlnorm(n=1000, meanlog $=\log (5.0), \operatorname{sdlog}=0.1)$

head(km.theta.vct)

\#Convert vector of simulated $\mathrm{Km}$ thetas to dataframe; assign column name

$\mathrm{km}$.theta $<$-data.frame $(\mathrm{km}$.theta.vet)

$\operatorname{dim}(\mathrm{km} . \mathrm{theta})$

names(km.theta)<-"KM_TH"

head(km.theta)

$\# * * * \# * * * \# * * * \# * * * \# * * * \# * * * \# * * * \#$

\#Read in Ln values of CLL, V1, V2, and Q thetas from 4 poppk studies

ln.cll.v1.v2.q.4mabs<-read.table("cll_v1_v2_q_ln theta_4 mabs.csv", header=T, sep=",", as.is $=\mathrm{T}$, skip $=0$ )

ln.cll.v1.v2.q.4mabs

\#Calculate correlation and covariance matrices for CLL, V1, V2, and Q

correlation<-cor(ln.cll.v1.v2.q.4mabs)

correlation

covariance $<-\operatorname{cov}(\ln . c 1 l . v 1 . v 2 . q .4 m a b s)$

covariance 
\#Export correlation and covariance matrices as .csv files

write.table(correlation, file="cll_v1_v2_q_correlation.csv", sep=",", col.names=T, row.names $=\mathrm{F}$ )

write.table(covariance, file="cll_v1_v2_q_covariance.csv", sep=",", col.names=T, row.names $=\mathrm{F}$ )

\#Load MASS package for mvrnorm function

library(MASS)

\#Create vector of means of Ln CLL, V1, V2, and Q thetas from 4 poppk studies \#Order is CLL, V1, V2, Q

$\mathrm{mu}<-\mathrm{c}(-3.926715,1.468283,1.003866,-3.212994)$

$\mathrm{mu}$

\#Simulate CLL (L/hr), V1 (L), V2 (L), and Q (L/hr) (Ln values) from multivariate \#normal distribution

multivariate. $\ln . \mathbf{v c t}<-\operatorname{mvrnorm}(\mathrm{n}=1000, \mathrm{mu}=\mathrm{mu}$, Sigma $=$ covariance $)$

head(multivariate.ln.vet)

\#Convert vector of CLL, V1, V2, and Q (Ln values) thetas to dataframe;

\#and assign column names

multivariate.ln<-data.frame(multivariate.ln.vet)

$\operatorname{dim}($ multivariate. $\ln )$

names(multivariate.ln)<-c("CLL_TH","V1_TH","V2_TH","Q_TH")

head(multivariate.ln)

\#Convert CLL, V1, V2, and Q Ln values to normal scale

multivariate.theta<-exp(multivariate. $\ln )$

head(multivariate.theta)

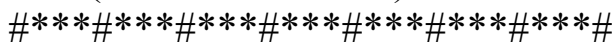

\#Simulate thetas for body weight effect on Vmax, CLL, V1, and V2

\#from uniform distributions; assign column names; and combine into \#one dataframe

wt.vmax.cll.vet $<-$ runif $(\mathrm{n}=1000, \min =0.4, \max =1.0)$

head(wt.vmax.cll.vct)

wt.vmax <-data.frame(wt.vmax.cll.vet) 


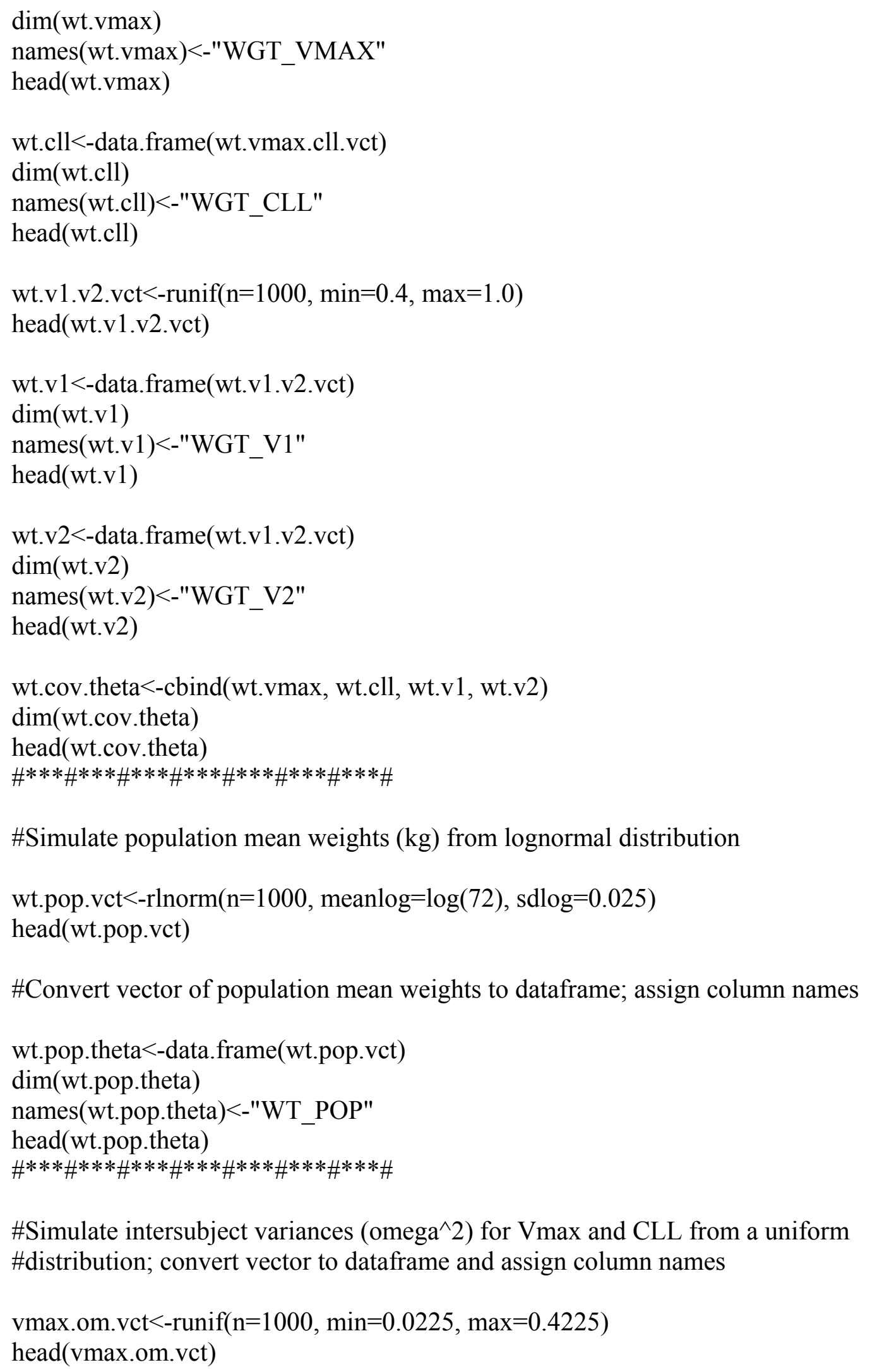

\#Simulate population mean weights $(\mathrm{kg})$ from lognormal distribution

wt.pop.vet $<$-rlnorm $(n=1000$, meanlog $=\log (72), \operatorname{sdlog}=0.025)$

head(wt.pop.vct)

\#Convert vector of population mean weights to dataframe; assign column names

wt.pop.theta $<$-data.frame(wt.pop.vet)

$\operatorname{dim}$ (wt.pop.theta)

names(wt.pop.theta)<-"WT_POP"

head(wt.pop.theta)

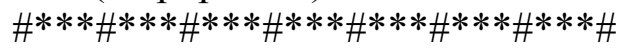

\#Simulate intersubject variances (omega $\left.{ }^{\wedge} 2\right)$ for Vmax and CLL from a uniform \#distribution; convert vector to dataframe and assign column names

vmax.om.vet<-runif $(\mathrm{n}=1000, \min =0.0225, \max =0.4225)$

head(vmax.om.vct) 


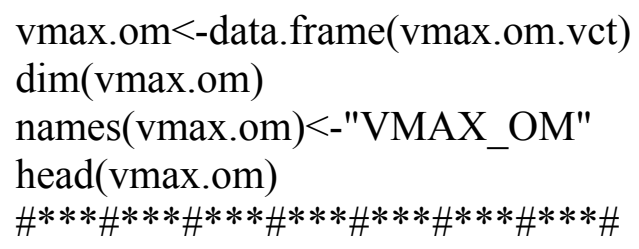

\#Simulate intersubject variances (omega^2) for V1 and V2 from a lognormal \#distribution; convert vector to dataframe and assign column names

v1.om.vct<-rlnorm(n=1000, meanlog $=\log (0.0625)$, sdlog $=0.4)$

head(v1.om.vct)

v1.om<-data.frame(v1.om.vet)

$\operatorname{dim}(\mathrm{v} 1.0 \mathrm{om})$

names(v1.om)<-"V1_OM"

head(v1.om)

$\# * * * \# * * * \# * * * \# * * * \# * * * \# * * * \# * * * \#$

v2.om.vet<-rlnorm(n=1000, meanlog $=\log (0.0625)$, sdlog=0.4)

head(v2.om.vet)

v2.om<-data.frame(v2.om.vct)

$\operatorname{dim}(\mathrm{v} 2 . \mathrm{om})$

names(v2.om)<-"V2_OM"

head(v2.om)

$\# * * * \# * * * \# * * * \# * * * \# * * * \# * * * \# * * * \#$

\#Create vector of 1000 intersubject variances (omega $\left.{ }^{\wedge} 2\right)$ for weight; fix \# at a variance of 0.04 , convert vector to dataframe and assign column name

wtpop.om.vct<-rep $(0.04,1000)$

head(wtpop.om.vct)

wtpop.om<-data.frame(wtpop.om.vet)

$\operatorname{dim}$ (wtpop.om)

names(wtpop.om)<-"WTPOP_OM"

head(wtpop.om) 
$\# * * * \# * * * \# * * * \# * * * \# * * * \# * * * \# * * * \#$

\#Simulate residual error variance $\left(\operatorname{sigma}^{\wedge} 2\right)$ from a lognormal distribution; \#convert vector to dataframe and assign column names

sigma.vct<-rlnorm(n=1000, meanlog $=\log (0.0225)$, sdlog $=0.4)$

head(sigma.vct)

sigma $<$-data.frame(sigma.vct)

$\operatorname{dim}(\operatorname{sigma})$

names( sigma)<-"SIGMA"

head(sigma)

$\# * * * \# * * * \# * * * \# * * * \# * * * \# * * * \# * * * \#$

\#Pull together dataframes into one large dataframe and export to .csv file \#Each row will contain one set of parameters to be used for each simulation \#of the data

$\operatorname{ls}()$

sim<-cbind(wt.pop.theta, vmax.theta, km.theta, multivariate.theta, wt.cov.theta, wtpop.om, vmax.om, cll.om, v1.om, v2.om, sigma) $\operatorname{dim}(\operatorname{sim})$ head $(\operatorname{sim})$

\#Change number of sig digits in the output simparam $<$-signif( $\operatorname{sim}$, digits $=4)$

$\operatorname{dim}($ simparam)

head(simparam)

write.table(simparam, file="simparam.csv", col.names=T, row.names=F, sep=",") 
B-2. Example R script ("trialsimulation.R") used to automate creation of replicate NONMEM control streams for simulation of the replicate data sets.

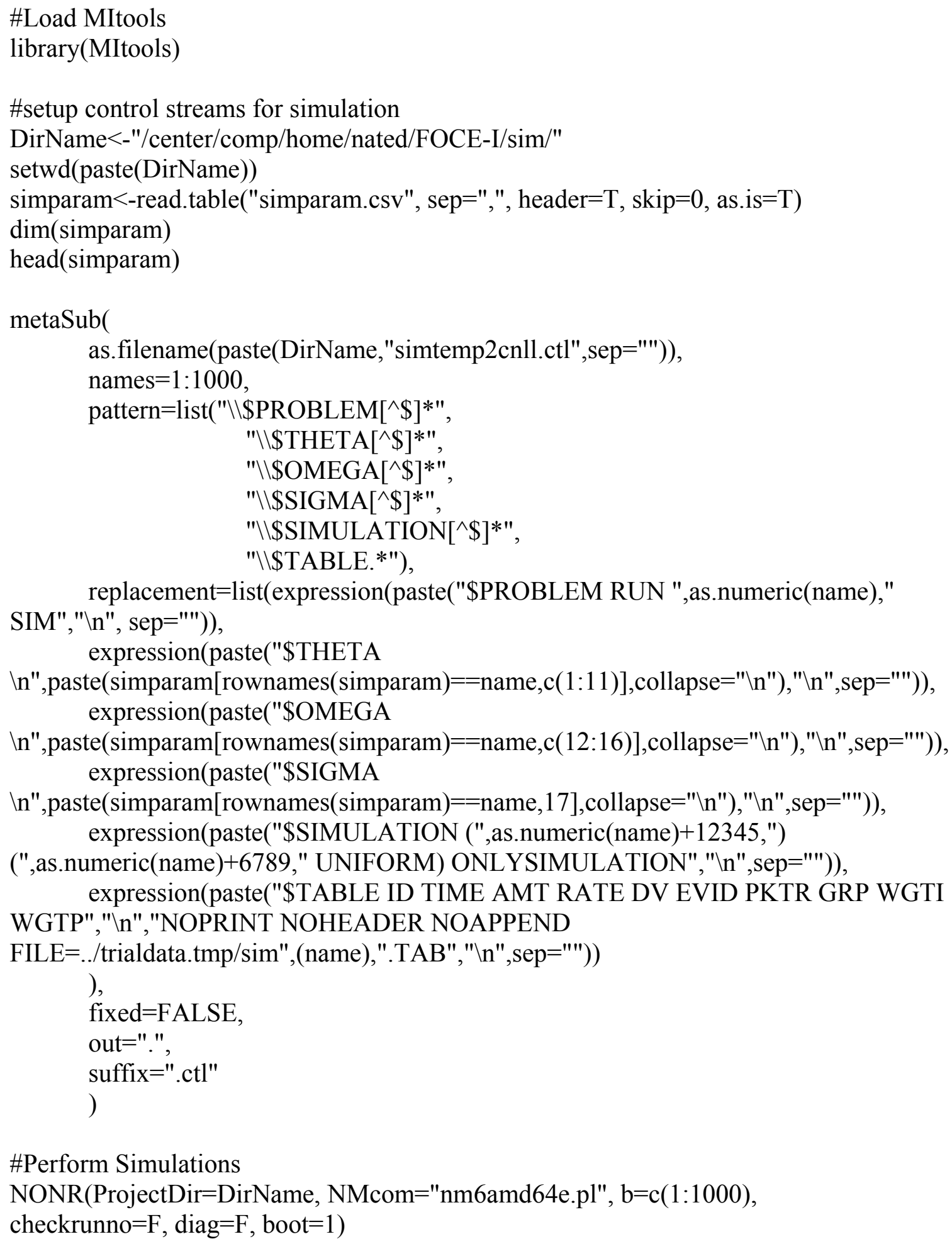

\#Perform Simulations

NONR(ProjectDir=DirName, NMcom="nm6amd64e.pl", b=c(1:1000), checkrunno $=\mathrm{F}$, diag $=\mathrm{F}$, boot $=1$ ) 
B-3. Example NONMEM control stream template ("simtemp2cnll.ctl") used for simulation of the replicate data sets. The R script in B-2 was executed using this control stream.

\$PROBLEM Control stream template for simulation of study replicates ; This will serve as the input for metaSUB, which will replace the ;thetas, omegas, sigmas, etc., with simulated parameter values. ; The substituted control stream will then be used to simulate data ;for one study (nrep=1)

\$INPUT C ID TIME AMT RATE DV EVID PKTR GRP

\$DATA ../studytemplate.csv IGNORE $=\mathrm{C}$ \$SUBROUTINE ADVAN6 TRANS1 TOL $=8$

$\$ M O D E L$ NPAR $=11$ NCOMP $=2$ COMP $=($ CENTRAL DEFOBSERVATION

DEFDOSE) $\mathrm{COMP}=(\mathrm{PERIPH})$

\$PK

WGTP $=$ THETA $(1)$

WGTI $=$ WGTP*EXP(ETA(1))

TVVMAX $=$ THETA $(2) *(\text { WGTI/72) })^{* * T H E T A(8)}$

$\mathrm{VMAX}=\mathrm{TVVMAX} * \operatorname{EXP}(\operatorname{ETA}(2))$

$\mathrm{KM}=\mathrm{THETA}(3)$

TVCLL $=$ THETA $(4) *($ WGTI $/ 72) * * \operatorname{THETA}(9)$

$\mathrm{CLL}=\mathrm{TVCLL} * \mathrm{EXP}(\operatorname{ETA}(3))$

TVV1 $=$ THETA $(5) *($ WGTI/72)**THETA $(10)$

$\mathrm{V} 1=\mathrm{TVV} 1 * \operatorname{EXP}(\operatorname{ETA}(4))$

TVV2 $=$ THETA $(6) *($ WGTI/72) $* * \operatorname{THETA}(11)$

$\mathrm{V} 2=\mathrm{TVV} 2 * \operatorname{EXP}(\operatorname{ETA}(5))$

$\mathrm{Q}=\operatorname{THETA}(7)$

$\mathrm{S} 1=\mathrm{V} 1$

\$DES

$\mathrm{C} 1=\mathrm{A}(1) / \mathrm{V} 1$

$\mathrm{C} 2=\mathrm{A}(2) / \mathrm{V} 2$

$\operatorname{DADT}(1)=-\mathrm{VMAX} * \mathrm{C} 1 /(\mathrm{KM}+\mathrm{C} 1)-(\mathrm{CLL} * \mathrm{C} 1)-(\mathrm{Q} * \mathrm{C} 1)+(\mathrm{Q} * \mathrm{C} 2)$

$\operatorname{DADT}(2)=(\mathrm{Q} * \mathrm{C} 1)-(\mathrm{Q} * \mathrm{C} 2)$

\$ERROR

$\mathrm{Y}=\mathrm{F}^{*} \mathrm{EXP}(\operatorname{EPS}(1))$ 


\section{\$THETA}

72.0 ;WTPOP (KG)

$0.75 ; \mathrm{VMAX}(\mathrm{MG} / \mathrm{HR})$

$5.0 ; \mathrm{KM}(\mathrm{MG} / \mathrm{L})$

$0.02 ; \mathrm{CLL}(\mathrm{L} / \mathrm{HR})$

$4.5 ; \mathrm{V} 1(\mathrm{~L})$

$3.0 ; \mathrm{V} 2(\mathrm{~L})$

$0.05 ; \mathrm{Q}(\mathrm{L} / \mathrm{HR})$

0.7 ;WGT $\sim$ VMAX

0.7 ;WGT $\sim \mathrm{CLL}$

$0.7 ; \mathrm{WGT} \sim \mathrm{V} 1$

$0.7 ; \mathrm{WGT} \sim \mathrm{V} 2$

\$OMEGA

0.04 ;WTPOP OM

0.04 ;VMAX OM

$0.04 ;$ CLL OM

$0.04 ; \mathrm{V} 1 \mathrm{OM}$

$0.04 ; \mathrm{V} 2 \mathrm{OM}$

\$SIGMA

0.04 ;EXPONENTIAL ERROR

\$SIMULATION (12345) (6789 UNIFORM) ONLYSIMULATION

\$TABLE ID TIME AMT RATE DV EVID PKTR GRP WGTI WGTP

NOPRINT NOHEADER NOAPPEND FILE=sim.TAB 
B-4. Example R script ("estctl_FOCEI_boot1.R") for setting up and executing the batch estimation runs with NONMEM.

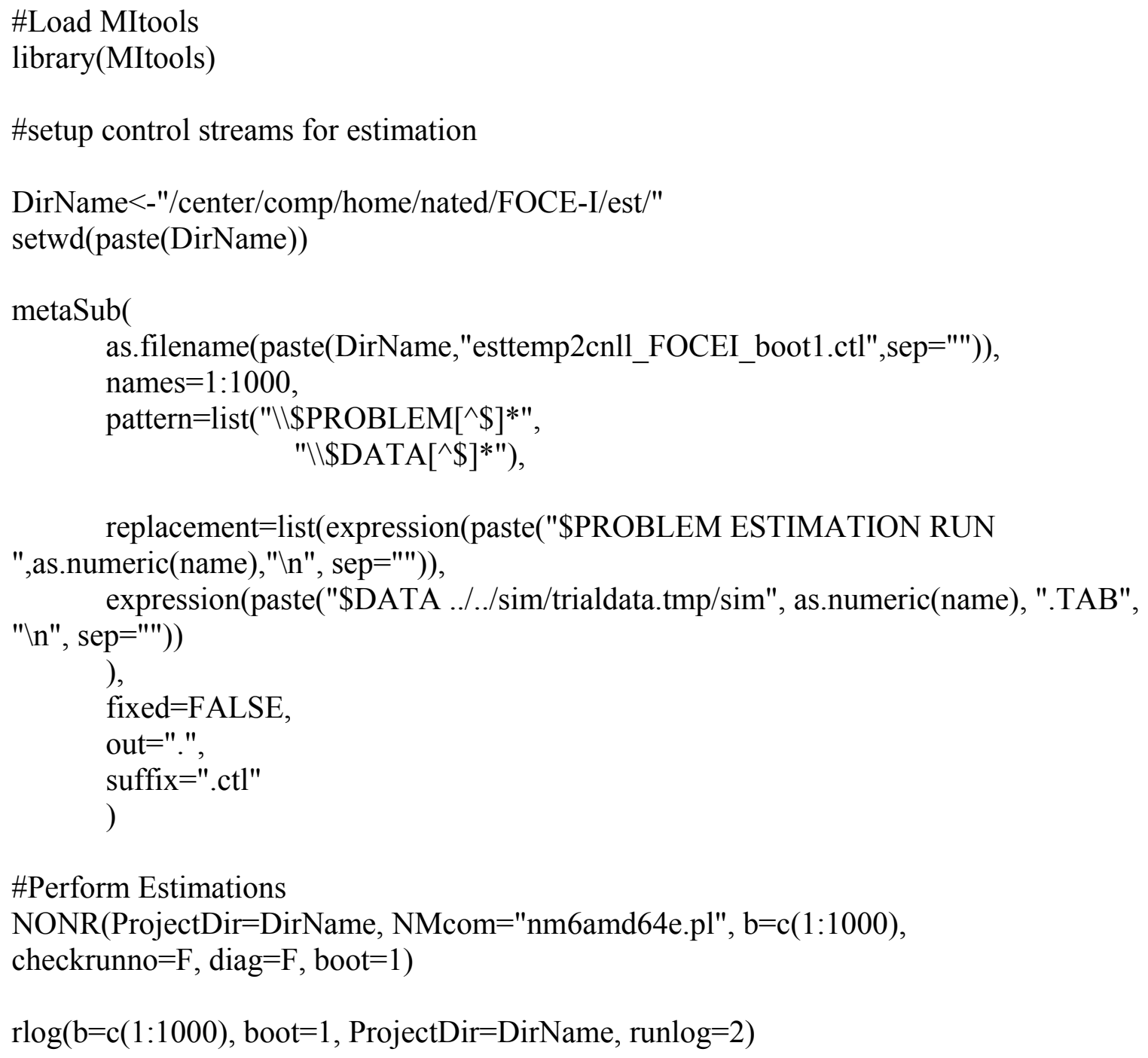


B-5. Example NONMEM control stream estimation template for the batch runs ("esttemp2cnll_FOCEI_boot1.ctl"). The R script in B-4 was executed using this control stream.

\$PROBLEM Control stream template for estimation runs \$INPUT ID TIME AMT RATE DV EVID PKTR GRP WGTI WGTP \$DATA .././sim/trialdata.tmp/sim\#.TAB \$SUBROUTINE ADVAN6 TRANS1 TOL=8 INFN=../MItoolsboot.for $\$$ MODEL NPAR $=10$ NCOMP $=2$ COMP $=($ CENTRAL DEFOBSERVATION DEFDOSE) $\mathrm{COMP}=(\mathrm{PERIPH})$

\$PK

TVVMAX $=$ THETA $(1) *(\text { WGTI/72) })^{*}$ THETA(7)

VMAX $=$ TVVMAX $*$ EXP(ETA(1))

$\mathrm{KM}=\operatorname{THETA}(2)$

TVCLL $=\operatorname{THETA}(3) *(\text { WGTI/72) })^{* * \operatorname{THETA}(8)}$

$\mathrm{CLL}=\mathrm{TVCLL} * \operatorname{EXP}(\operatorname{ETA}(2))$

TVV1 $=$ THETA $(4) *($ WGTI/72)**THETA $(9)$

$\mathrm{V} 1=\mathrm{TVV} 1 * \operatorname{EXP}(\operatorname{ETA}(3))$

TVV2 $=$ THETA $(5) *($ WGTI/72)**THETA(10)

$\mathrm{V} 2=\mathrm{TVV} 2 * \operatorname{EXP}(\operatorname{ETA}(4))$

$\mathrm{Q}=\mathrm{THETA}(6)$

$\mathrm{S} 1=\mathrm{V} 1$

\$DES

$\mathrm{C} 1=\mathrm{A}(1) / \mathrm{V} 1$

$\mathrm{C} 2=\mathrm{A}(2) / \mathrm{V} 2$

DADT $(1)=-\mathrm{VMAX} * \mathrm{C} 1 /(\mathrm{KM}+\mathrm{C} 1)-(\mathrm{CLL} * \mathrm{C} 1)-(\mathrm{Q} * \mathrm{C} 1)+(\mathrm{Q} * \mathrm{C} 2)$

$\operatorname{DADT}(2)=\left(\mathrm{Q}^{*} \mathrm{C} 1\right)-(\mathrm{Q} * \mathrm{C} 2)$

\$ERROR

$\mathrm{Y}=\mathrm{F} * \operatorname{EXP}(\operatorname{EPS}(1))$

\$THETA

$(0,1.2)$; VMAX (MG/HR)

$(0,10) ; \mathrm{KM}(\mathrm{MG} / \mathrm{L})$

$(0,0.03)$; CLL (L/HR)

$(0,3) ; \mathrm{V} 1(\mathrm{~L})$

$(0,2) ; \mathrm{V} 2(\mathrm{~L})$ 
$(0,0.075) ; \mathrm{Q}(\mathrm{L} / \mathrm{HR})$

$0.8 ;$ WGT $\sim$ VMAX

$0.8 ; \mathrm{WGT} \sim \mathrm{CLL}$

$0.8 ; \mathrm{WGT} \sim \mathrm{V} 1$

$0.8 ; \mathrm{WGT} \sim \mathrm{V} 2$

\$OMEGA

$0.16 ; \mathrm{VMAX}$ OM

$0.16 ;$ CLL OM

$0.16 ; \mathrm{V} 1 \mathrm{OM}$

$0.16 ; \mathrm{V} 2 \mathrm{OM}$

\$SIGMA

0.16 ;EXPONENTIAL ERROR

\$ESTIMATION METHOD=1 INTERACTION NOABORT MAXEVAL=9999

$\mathrm{PRINT}=5$

NOTBT NOOBT NOSBT

\$COVARIANCE

;END 
B-6. Section of the BUGSModelLibrary model file ("TwoCptParallelModelRK45.odc") specifying the differential equations for the twocompartment PK model with parallel nonlinear and linear elimination pathways.

MODULE PmetricsTwoCptParallelModelRK45;

IMPORT

PmetricsPKModels,

Math,

MathODE,

MathRungeKutta45,

Vec := LibVectors,

Solve := LibSolve;

TYPE

Equations $=$ POINTER TO RECORD(MathODE.Equations) END;

ODEModel* ${ }^{*}$ POINTER TO RECORD (PmetricsPKModels.PKModel)

END;

SSFun $=$ POINTER TO RECORD (Solve.RouteFns)

m: PmetricsPKModels.PKModel;

p: PmetricsPKModels.ModelParameters;

amt, rate, tau: REAL;

cmt: INTEGER

END;

CONST tol $=1.0 \mathrm{E}-7$

numEq $=2$;

VAR

equations: Equations;

solver: MathODE.Solver;

PROCEDURE UserDerivatives(IN theta, $\mathrm{x}$ : ARRAY OF REAL; numEq: INTEGER; t: REAL; OUT dxdt: ARRAY OF REAL) ;

VAR BEGIN

Vmax, Km, CLL, V1, V2, Q, C1, C2: REAL;

$\operatorname{Vmax}:=$ theta[0];

CLL := theta[1];

$\mathrm{V} 1:=$ theta[2];

$\mathrm{V} 2:=$ theta[3];

$\mathrm{Km}:=$ theta[4];

$\mathrm{Q}:=$ theta[5];

$\operatorname{ASSERT}((\operatorname{Vmax}>0) \&(\mathrm{Km}>0) \&(\mathrm{CLL}>0) \&(\mathrm{~V} 1>0) \&(\mathrm{~V} 2>0) \&$

$(\mathrm{Q}>0), 20)$ 
$\mathrm{C} 1:=\mathrm{x}[0] / \mathrm{V} 1$
$\mathrm{C} 2:=\mathrm{x}[1] / \mathrm{V} 2$

(* Differential equations for the model excluding piecewise constant input rates provided in the data set $*$ )

$$
\begin{aligned}
& \operatorname{dxdt}[0]:=-\mathrm{Vmax} * \mathrm{C} 1 /(\mathrm{Km}+\mathrm{C} 1)-\mathrm{CLL}{ }^{*} \mathrm{C} 1-\mathrm{Q}^{*} \mathrm{C} 1+\mathrm{Q} * \mathrm{C} 2 \\
& \operatorname{dxdt}[1]:=\mathrm{Q}
\end{aligned}
$$

END UserDerivatives;

PROCEDURE (equations: Equations) Derivatives* (IN theta, x: ARRAY OF

\section{REAL;}

numEq: INTEGER; t: REAL; OUT dxdt: ARRAY OF REAL);

VAR

BEGIN

i, iRate: INTEGER;

UserDerivatives (theta, $\mathrm{x}$, numEq, $\mathrm{t}$, dxdt);

iRate $:=\mathrm{LEN}$ (theta) - numEq;

FOR $\mathrm{i}:=0$ TO numEq-1 DO

$$
\mathrm{dxdt}[\mathrm{i}]:=\mathrm{dxdt}[\mathrm{i}]+\text { theta[iRate }+\mathrm{i}]
$$

END;

END Derivatives;

PROCEDURE (equations: Equations) SecondDerivatives* (IN theta, x: ARRAY OF REAL; BEGIN

END SecondDerivatives;

PROCEDURE (equations: Equations) Jacobian* (IN theta, x: ARRAY OF REAL; numEq: INTEGER; t: REAL; OUT jacob: ARRAY OF ARRAY OF REAL);

BEGIN

END Jacobian;

PROCEDURE (m: ODEModel) InitModel*;

BEGIN

m.nParameter $:=10$;

m.F1Index $:=6$;

m.tlag1Index $:=8$;

m.nCmt :=2;

MathRungeKutta45.Install;

solver := MathRungeKutta45.fact.New();

NEW(equations); 
solver.Init(equations, numEq); END InitModel; 
B-7. Example R script (“1OSD_TwoCptParallelModelUP.R”) used in conjunction with the Bayesian model with uninformative priors. This R script was used for data management, launching WinBUGS, and analysis of the MCMC samples.

\# WinBUGS PKPD Model Library: Two compartment PK model with nonlinear/linear elimination pathways and infusion input

if(.Platform\$OS.type == "windows") Sys.setenv(HOME = "c:")

\#subdirectory containing the BUGS model file

\#problemDir = file.path $($ Sys.getenv $(" H O M E ")$,

"metrum/Institute/nateDirks/nate02032009")

problemDir = "C:/BUGSModelLibrary" \#

toolsDir $=$ problemDir

\#toolsDir = file.path(Sys.getenv("HOME"), "bugsTools")

model.name $=$ "TwoCptParallelModelUP_RK45" \# root names of model file

bugsDir = "C:/Program Files (x86)/BlackBox Component Builder 1.5/"

\#bugsDir = file.path(Sys.getenv("HOME"), "Program Files/BlackBoxWinBUGS/")

wineBin = "/usr/local/MacPorts/bin" \# directory containing wine and winepath programs. only relevant on unix or Mac OS X

setwd(problemDir)

\#library(R2WinBUGS)

library(coda)

library(lattice)

source(file.path(toolsDir,"bugs.tools.R"))

source(file.path(toolsDir,"bgillespie.utilities.R"))

source(file.path(toolsDir,"myR2WinBUGS.R"))

set.seed(10271998) \# not required but assures repeatable results

options $($ error $=$ expression(NULL)) \# prevents stopping for errors when running in batch memory.limit(2048)

\# get data files

datname $=" 1 "$

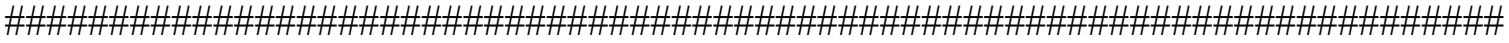

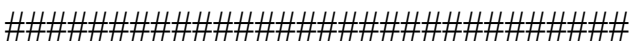

predata $=$ read.table("C:/BUGSModelLibrary/OSD_Replicates/sim1.TAB", header=F,

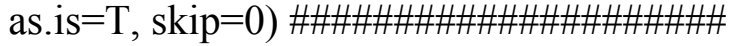

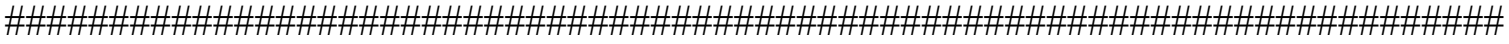

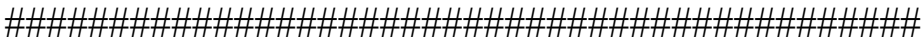

data $=$ predata $[, c(1: 6,9)]$

names(data) = c("subject","time","amt","rate","dv","evid","weight")

data $\$ \mathrm{dv}[$ data $\$$ evid==1] = "."

data $\$ \mathrm{dv}=$ as.numeric $(\mathrm{data} \$ \mathrm{dv})$

data $\$$ addl $=\operatorname{rep}(0, \operatorname{nrow}($ data $))$ 


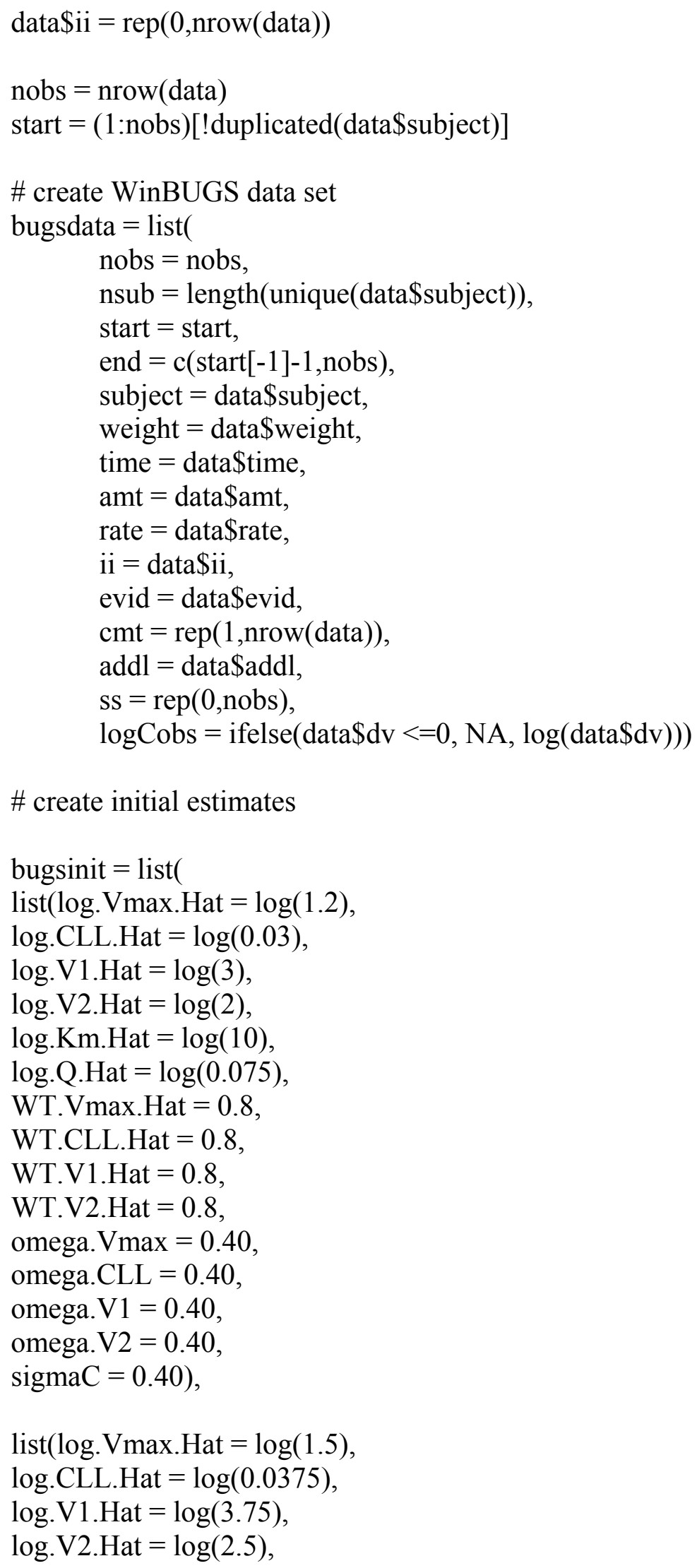




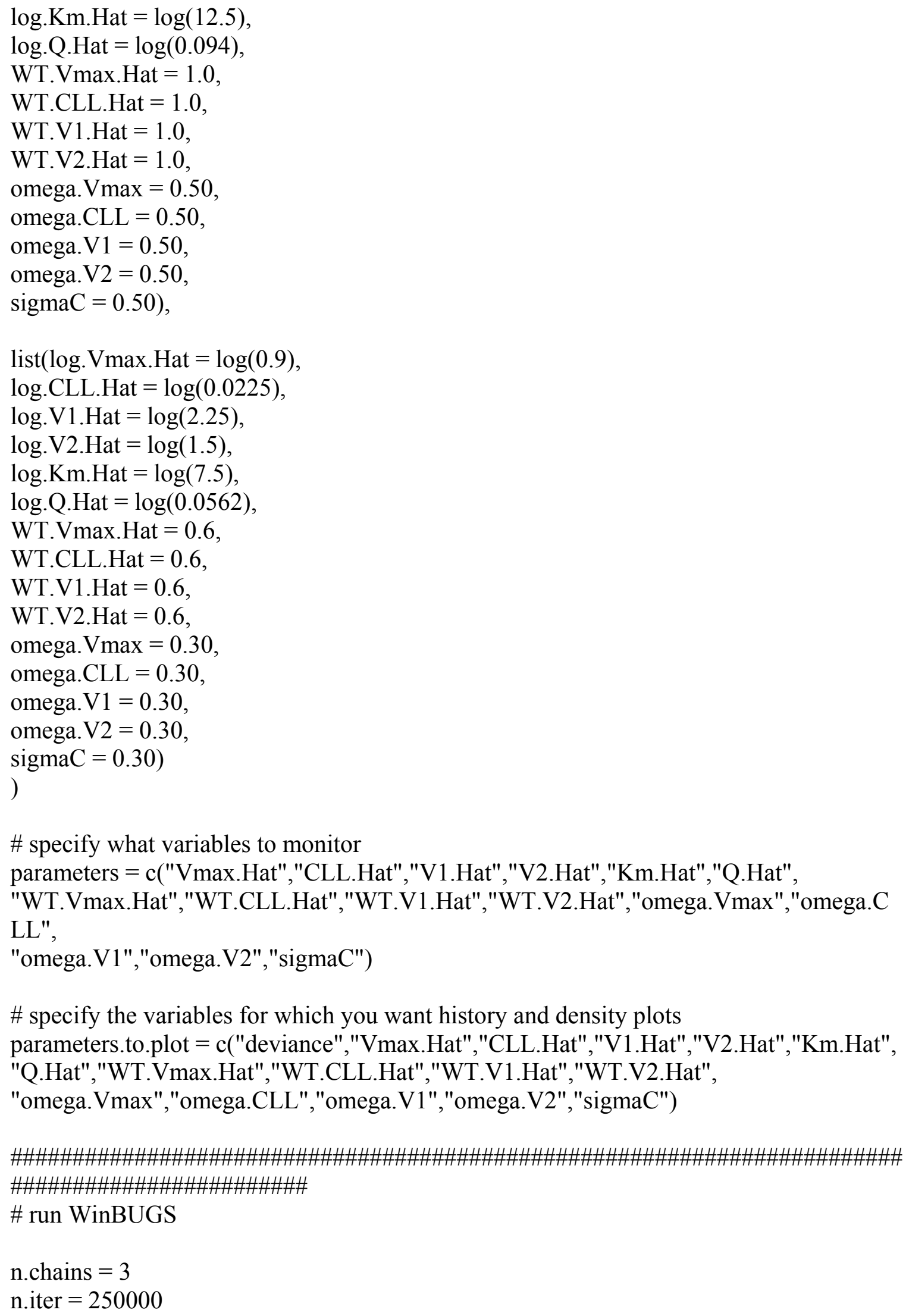


n.burnin $=25000$

n.thin $=25$

bugs.fit $=$ my.bugs $($ data $=$ bugsdata,inits $=$ bugsinit,parameters.to.save= $=$ parameters, model.file=file.path(getwd(),paste(model.name,".txt",sep=""')), n.chains $=$ n.chains, $n$. iter $=$ n.iter,n.burnin $=$ n.burnin, $n$.thin $=$ n.thin, clearWD $=F$, bugs.directory $=$ bugsDir, refresh $=1$, useWINE=(.Platform\$OS.type == "unix"), WINE=file.path(wineBin,"wine"), newWINE $=(. P l a t f o r m \$ O S . t y p e==$ "unix" $)$,

WINEPATH=file.path(wineBin,"winepath"))

\# save scripts, data and results to a directory

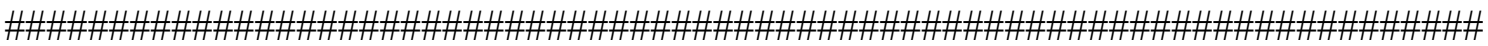
resultsdir $=$ "C:/BUGSModelLibrary/OSD_UninformativePrior_Results"

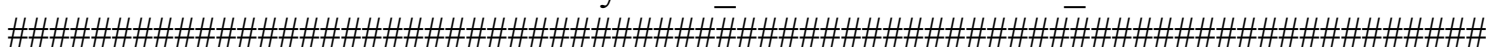

save.model(bugs.fit,paste(datname,"OSD","_",model.name,sep=""'))

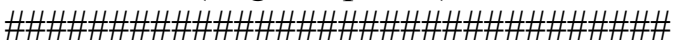

\#load(paste(model.name,"/",model.name,".fit.Rsave",sep="'))

\# rename and reformat MCMC results to facilitate later calculations and plots

sims.array $=$ bugs.fit\$sims.array

posterior $=$

array(as.vector(sims.array), $\operatorname{dim}=\mathrm{c}(\operatorname{prod}(\operatorname{dim}(\operatorname{sims} . \operatorname{array})[1: 2]), \operatorname{dim}(\operatorname{sims} . \operatorname{array})[3])$, dimnames $=$ list(NULL, dimnames(sims.array)[[3]]))

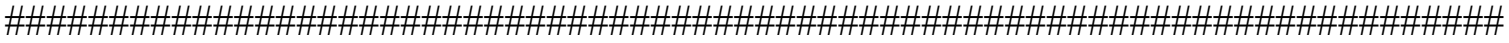

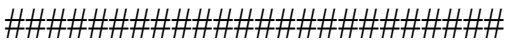

\# posterior distributions of parameters

\# open graphics device

pdf(file = paste(resultsdir,"/",datname,".plots.pdf",sep=""),width=6,height=6)

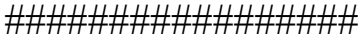

\# subset of sims.array containing selected variables

$\mathrm{x} 1$ = sims.array[,,unlist(sapply(c(paste("^",parameters.to.plot,"\$",sep=""), paste("^",parameters.to.plot,"\\[",sep=""')),grep,x=dimnames(sims.array)[[3]]))]

\# create history, density and Gelman-Rubin-Brooks plots, and a table of summary stats ptable $=$ parameter.plot.table $(\mathrm{x} 1)$

write.csv(signif(ptable,3),paste(resultsdir,"/",datname,".summary.csv",sep="'))

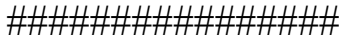




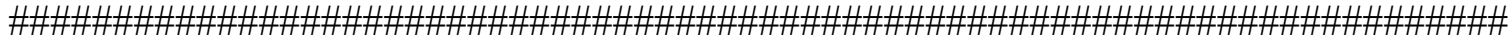

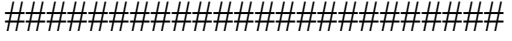

dev.off() 
B-8. BUGS language model for fitting the two-compartment parallel elimination PK model using uninformative priors ("TwoCptParallelModelUP_RK45.txt").

model

\{

for(i in $1:$ nsub $)\{$

logtheta[i, 1] dnorm(logthetaMean[i, 1], omega.inv.Vmax)

\#Vmax eta

logtheta[i, 2] dnorm(logthetaMean[i, 2], omega.inv.CLL)

\#CLL eta

logtheta[i, 3] dnorm(logthetaMean[i, 3], omega.inv.V1)

\#V1 eta

logtheta[i, 4] dnorm(logthetaMean[i, 4], omega.inv.V2)

\#V2 eta

logtheta $[i, 5]<-$ logthetaMean[i, 5]

\#Km fixed

logtheta $[i, 6]<-$ logthetaMean $[i, 6]$

\#Q fixed

logthetaMean $[\mathrm{i}, 1]<-$

log.Vmax.Hat + WT.Vmax.Hat*log(weight[start[i]]/72) \#Vmax TH

logthetaMean $[\mathrm{i}, 2]<-$

log.CLL.Hat + WT.CLL.Hat* $\log ($ weight[start[i]]/72) \#CLL TH

logthetaMean[i, 3]<-

log.V1.Hat + WT.V1.Hat* $\log ($ weight[start[i]]/72) \#V1 TH

logthetaMean $[\mathrm{i}, 4]<-$

log.V2.Hat + WT.V2.Hat*log(weight[start[i]]/72) \#V2 TH

logthetaMean $[\mathrm{i}, 5]<-$

$\log . \mathrm{Km} . \mathrm{Hat}$

\#KM TH

logthetaMean $[i, 6]<-$

log.Q.Hat

\#Q TH 


$$
\begin{aligned}
& \text { theta[i,7]<- } 1 \text { \# F1 } \\
& \text { theta[i,8]<- } 1 \text { \# F2 } \\
& \text { theta[i,9]<- } 0 \text { \# tlag1 } \\
& \text { theta[i,10]<- } 0 \text { \# tlag2 } \\
& \text { for }(j \text { in } 1: 6)\{ \\
& \qquad \log (\operatorname{theta}[i, j])<-\operatorname{logtheta}[i, j] \\
& \text { xhat[start[i]:end[i],1:2]<- }
\end{aligned}
$$

TwoCptParallelModelRK45(time[start[i]:end[i]], amt[start[i]:end[i]], rate[start[i]:end[i]], ii[start[i]:end[i]], evid[start[i]:end[i]], cmt[start[i]:end[i]], addl[start[i]:end[i]], ss[start[i]:end[i]], theta[i,])

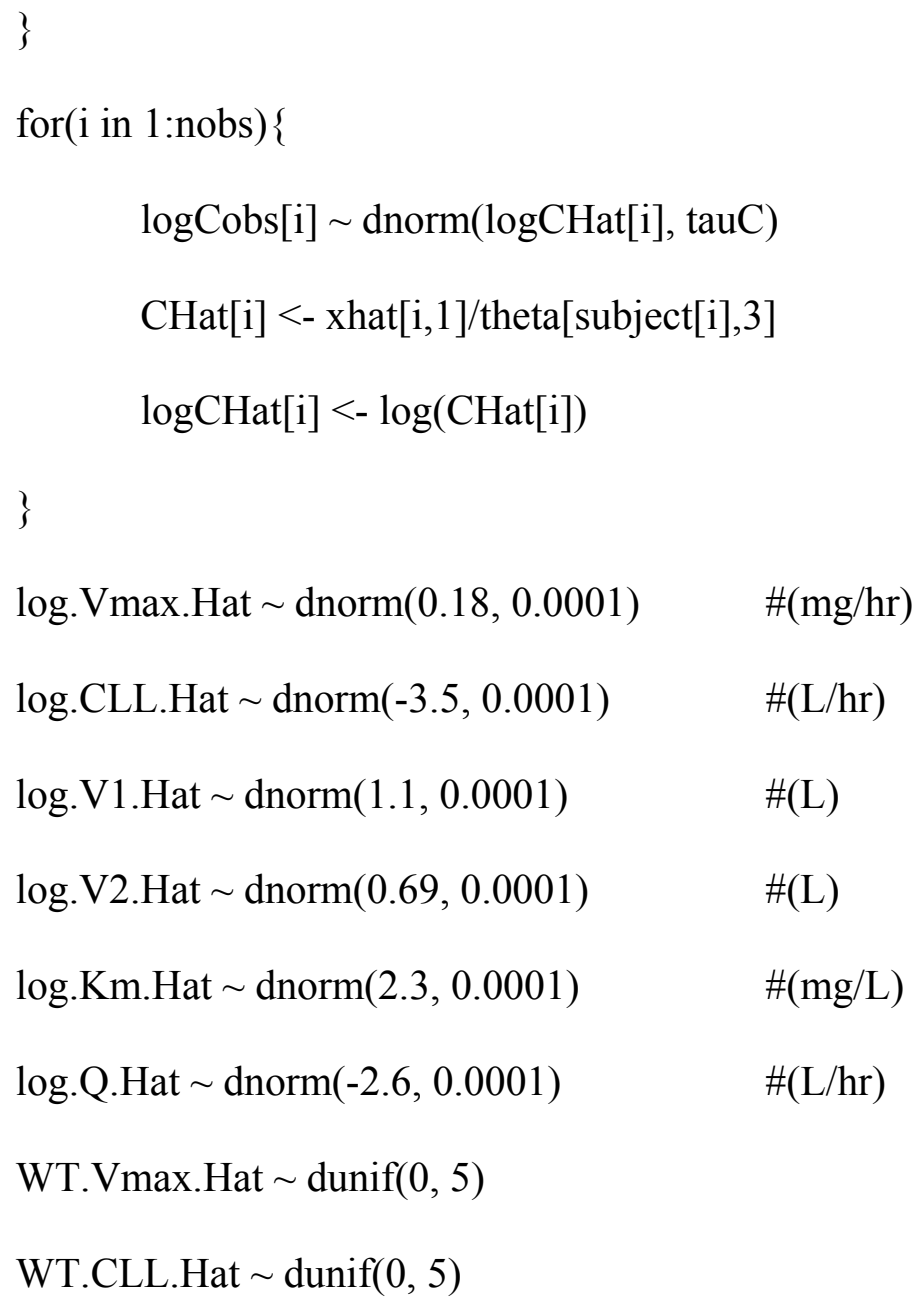




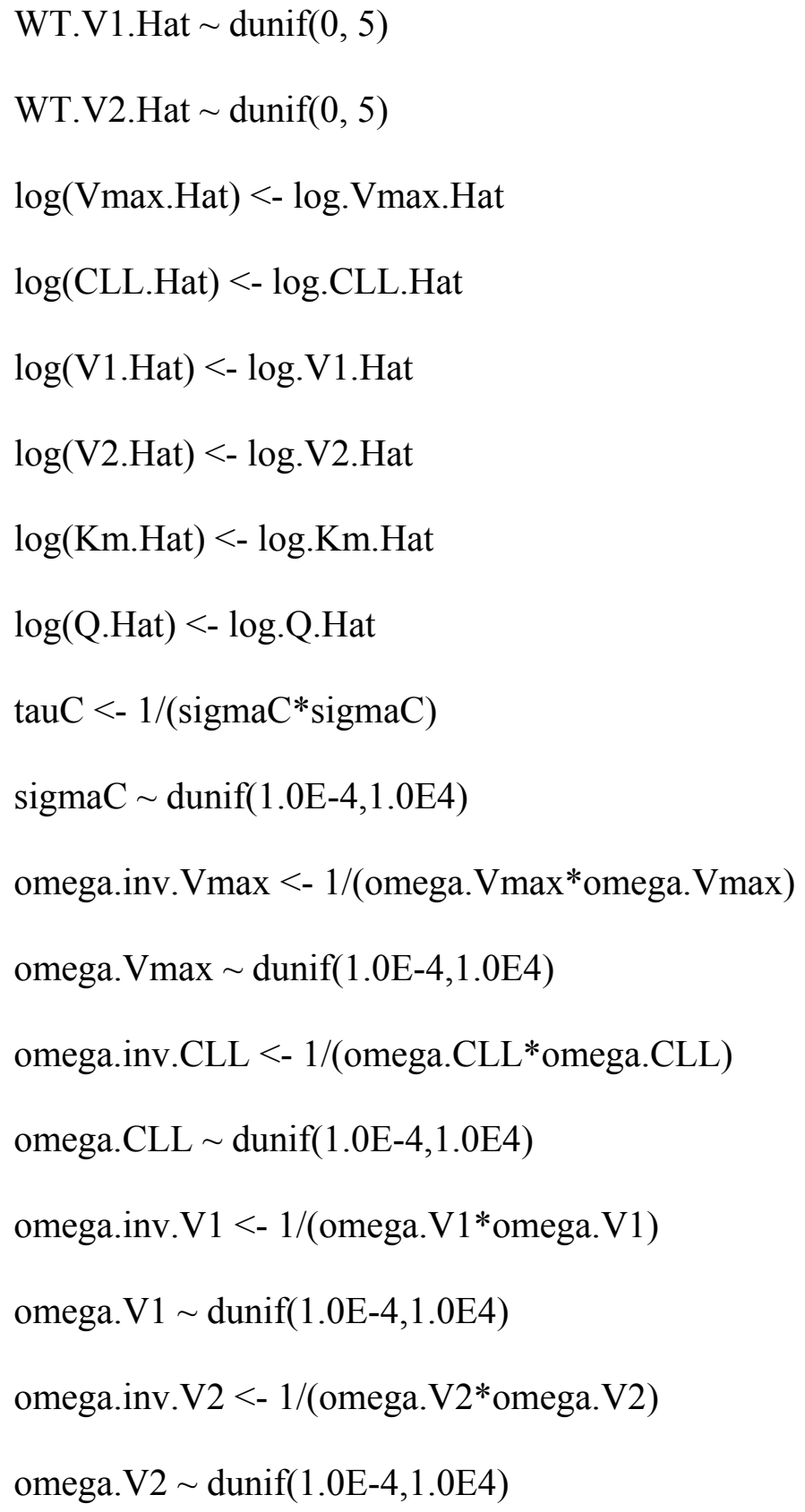


B-9. Example R script ("1OSD_TwoCptParallelModel_IP.R") used in conjunction with the Bayesian model with informative priors. This R script was used for data management, launching WinBUGS, and analysis of the MCMC samples.

\# WinBUGS PKPD Model Library: Two compartment PK model with nonlinear/linear elimination pathways and infusion input

if(.Platform\$OS.type == "windows") Sys.setenv(HOME = "c:")

\#subdirectory containing the BUGS model file

\#problemDir = file.path(Sys.getenv("HOME"),

"metrum/Institute/nateDirks/nate02032009")

problemDir = "C:/BUGSModelLibrary" \#

toolsDir $=$ problemDir

\#toolsDir = file.path(Sys.getenv("HOME"), "bugsTools")

model.name $=$ "TwoCptParallelModel_IP_RK45" \# root names of model file

bugsDir $=$ "C:/Program Files (x86)/BlackBox Component Builder 1.5/"

\#bugsDir = file.path(Sys.getenv("HOME"), "Program Files/BlackBoxWinBUGS/")

wineBin = "/usr/local/MacPorts/bin" \# directory containing wine and winepath programs. only relevant on unix or Mac OS X

setwd(problemDir)

\#library(R2WinBUGS)

library(coda)

library(lattice)

source(file.path(toolsDir,"bugs.tools.R"))

source(file.path(toolsDir,"bgillespie.utilities.R"))

source(file.path(toolsDir,"myR2WinBUGS.R"))

set.seed(10271998) \# not required but assures repeatable results

options $($ error $=$ expression(NULL)) \# prevents stopping for errors when running in batch memory.limit(2048)

\# get data files

datname $=" 1 "$

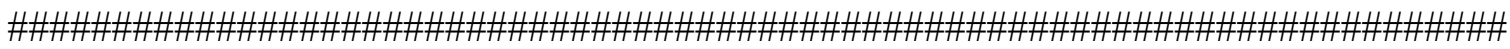

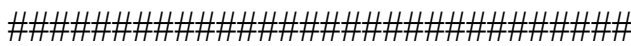

predata $=$ read.table("C:/BUGSModelLibrary/OSD_Replicates/sim1.TAB", header=F,

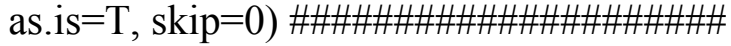

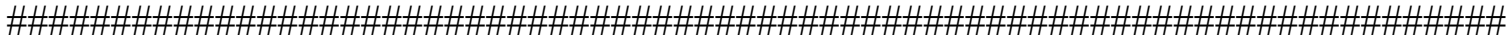

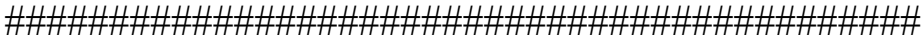

data $=\operatorname{predata}[, \mathrm{c}(1: 6,9)]$

names(data) = c("subject","time","amt","rate","dv","evid","weight")

data $\$ \mathrm{dv}[$ data $\$$ evid $==1]=" . "$

data $\$ \mathrm{dv}=$ as.numeric $(\mathrm{data} \$ \mathrm{dv})$

data $\$$ addl $=\operatorname{rep}(0, \operatorname{nrow}($ data $))$ 


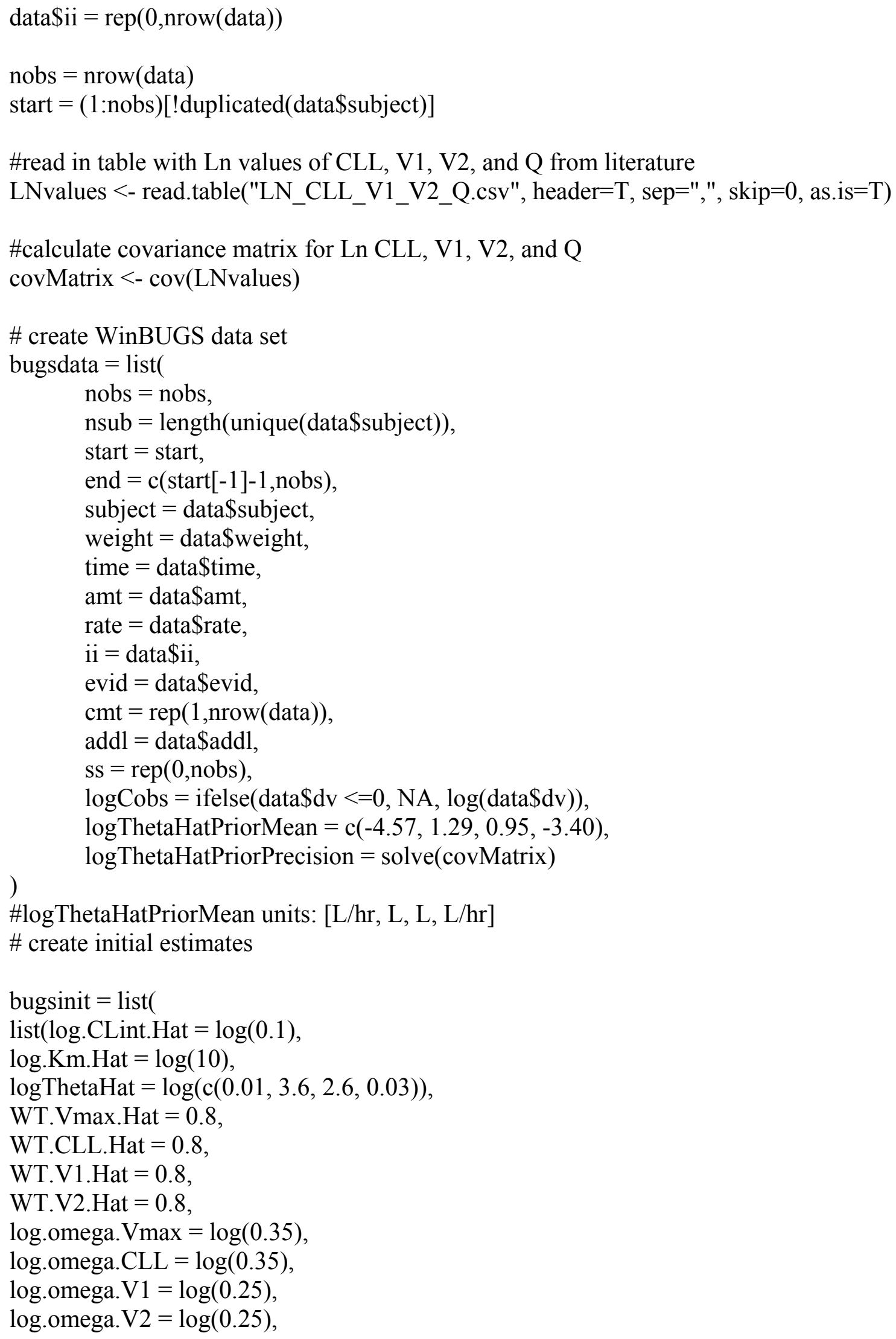




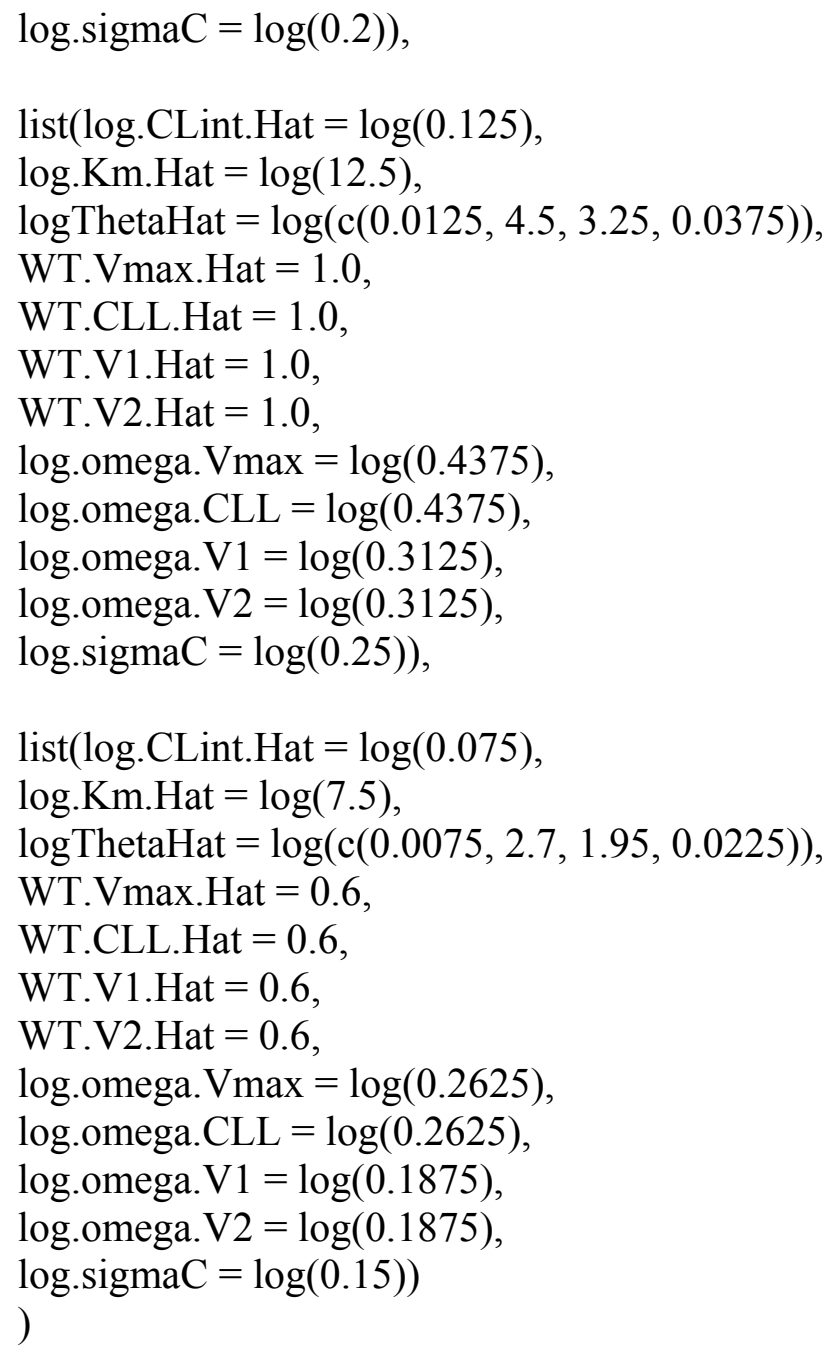


n.chains $=3$

n.iter $=250000$

n.burnin $=25000$

n.thin $=25$

bugs.fit $=$ my.bugs $($ data $=$ bugsdata,inits $=$ bugsinit,parameters. to.. save $=$ parameters, model.file=file.path(getwd(),paste(model.name,".txt",sep="")), n.chains $=$ n.chains,n.iter $=$ n.iter,n.burnin $=$ n.burnin, $n$. thin $=$ n.thin, clearWD $=F$, bugs.directory $=$ bugsDir, refresh $=1$, useWINE=(.Platform\$OS.type == "unix"), WINE=file.path(wineBin,"wine"), newWINE $=(. P l a t f o r m \$ O S . t y p e==$ "unix"),

WINEPATH=file.path(wineBin,"winepath"))

\# save scripts, data and results to a directory

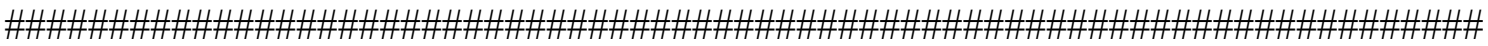
resultsdir $=$ "C:/BUGSModelLibrary/OSD_InformativePrior_Results"

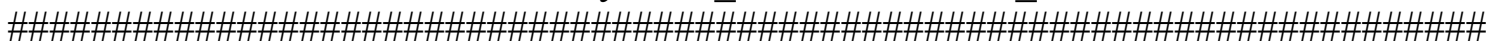

save.model(bugs.fit,paste(datname,"OSD","_",model.name,sep=""'))

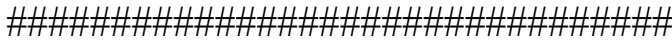

\#load(paste(model.name,"/",model.name,".fit.Rsave",sep=""))

\# rename and reformat MCMC results to facilitate later calculations and plots

sims.array $=$ bugs.fit\$sims.array

posterior $=$

$\operatorname{array}($ as.vector(sims.array), $\operatorname{dim}=\mathrm{c}(\operatorname{prod}(\operatorname{dim}(\operatorname{sims}$.array) $[1: 2]), \operatorname{dim}(\operatorname{sims} . \operatorname{array})[3])$, dimnames $=$ list(NULL, dimnames(sims.array)[[3]]))

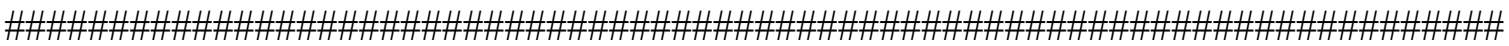

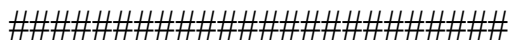

\# posterior distributions of parameters

\# open graphics device

pdf(file = paste(resultsdir,"/",datname,".plots.pdf",sep=""),width=6,height=6)

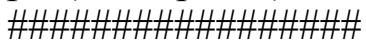

\# subset of sims.array containing selected variables

x1 = sims.array[,,unlist(sapply(c(paste("^",parameters.to.plot,"\$",sep=""), paste("^",parameters.to.plot,"\\[",sep="" )),grep,x=dimnames(sims.array)[[3]]))]

\# create history, density and Gelman-Rubin-Brooks plots, and a table of summary stats ptable $=$ parameter.plot.table $(\mathrm{x} 1)$ 
write.csv(signif(ptable,3),paste(resultsdir,"/",datname,".summary.csv",sep=""))

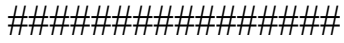

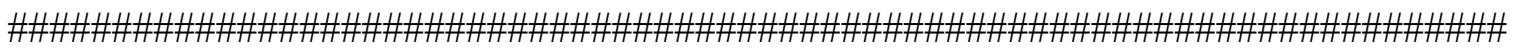

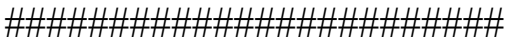

dev.off() 
B-10. BUGS language model for fitting the two-compartment parallel elimination PK model using informative priors (“TwoCptParallelModel_IP_RK45.txt”).

model

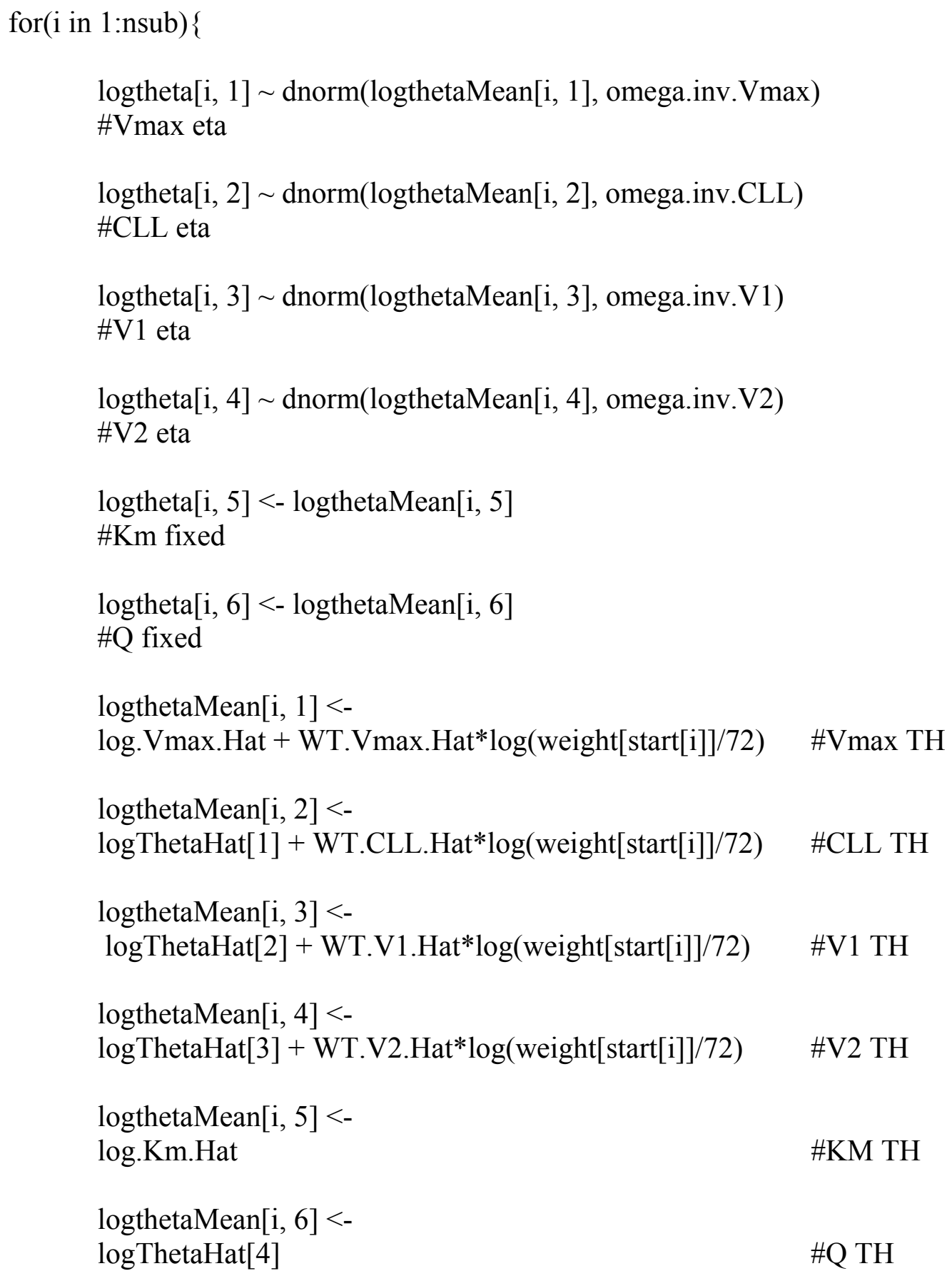




$$
\begin{aligned}
& \text { theta[i,7]<- } 1 \text { \# F1 } \\
& \text { theta[i,8]<- } 1 \text { \# F2 } \\
& \text { theta[i,9]<- } 0 \text { \# tlag1 } \\
& \text { theta[i,10]<- } 0 \text { \# tlag2 } \\
& \text { for }(j \text { in } 1: 6)\{ \\
& \qquad \log (\operatorname{theta}[i, j])<-\operatorname{logtheta}[i, j] \\
& \text { xhat }[\operatorname{start}[i]: \text { end }[i], 1: 2]<-
\end{aligned}
$$

TwoCptParallelModelRK45(time[start[i]:end[i]], amt[start[i]:end[i]], $\operatorname{rate[start[i]:end[i]],~}$ ii[start[i]:end[i]], evid[start[i]:end[i]], $\operatorname{cmt}[\operatorname{start}[i]: \operatorname{end}[i]]$, addl[start[i]:end[i]], ss[start[i]:end[i]], theta[i,])

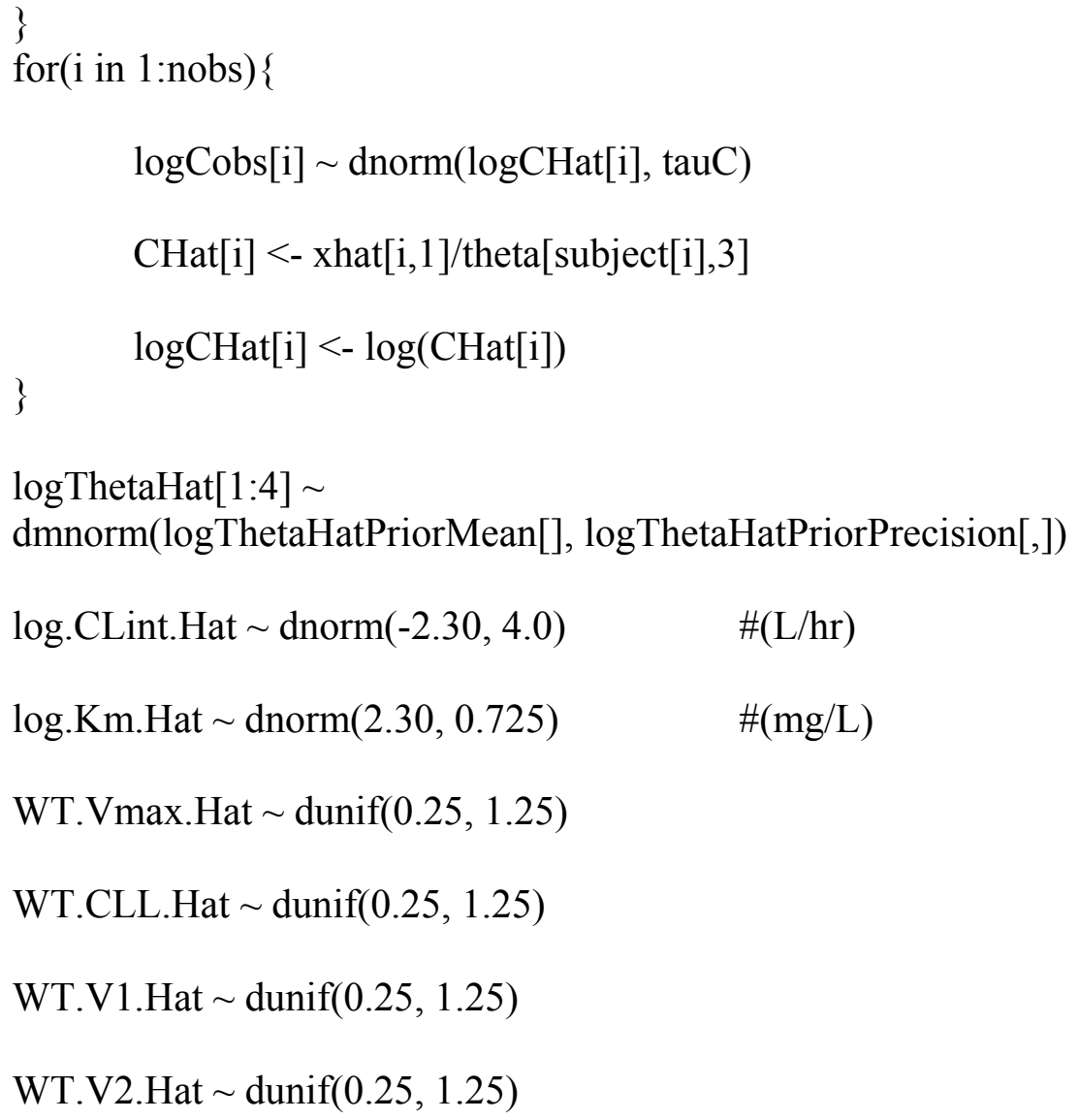




$$
\begin{aligned}
& \log (\text { CLint.Hat })<-\log \text {.CLint.Hat } \\
& \log (\text { CLL.Hat })<-\log \text { ThetaHat[1] } \\
& \log (\text { V1.Hat })<-\log \text { ThetaHat[2] } \\
& \log (\text { V2.Hat })<-\log \text { ThetaHat[3] } \\
& \log (\text { Km.Hat })<-\log . \text { Km.Hat } \\
& \log (\text { Q.Hat })<-\log \text { ThetaHat[4] } \\
& \text { Vmax.Hat }<- \text { Km.Hat*CLint.Hat } \\
& \log \text {.Vmax.Hat }<-\log (\text { Vmax.Hat }) \\
& \text { tauC }<-1 /(\operatorname{sigmaC} * \text { sigmaC }) \\
& \log (\operatorname{sigmaC})<-\log \cdot \operatorname{sigmaC} \\
& \log . \operatorname{sigmaC} \sim \operatorname{dnorm}(-1.61,6.25) \\
& \text { omega.inv.Vmax }<-1 /(\text { omega.Vmax*omega.Vmax) } \\
& \log (\text { omega.Vmax })<- \text { log.omega.Vmax } \\
& \text { log.omega.Vmax } \sim \operatorname{dnorm}(-1.05,6.25) \\
& \text { omega.inv.CLL <- 1/(omega.CLL*omega.CLL) } \\
& \log (\text { omega.CLL })<- \text { log.omega.CLL } \\
& \text { log.omega.CLL } \sim \text { dnorm(-1.05, 6.25) } \\
& \text { omega.inv.V1 <- 1/(omega.V1*omega.V1) } \\
& \log (\text { omega.V1) }<- \text { log.omega.V1 } \\
& \text { log.omega.V1 dnorm(-1.39, 6.25) } \\
& \text { omega.inv.V2 <- 1/(omega.V2*omega.V2) } \\
& \log (\text { omega.V2) }<- \text { log.omega.V2 } \\
& \text { log.omega.V2 } \sim \text { dnorm(-1.39, 6.25) }
\end{aligned}
$$


APPENDIX C:

CHAPTER 4 SUPPLEMENTAL FIGURES AND TABLES 
Table C-1. Bias (median percent estimation error) in the population pharmacokinetic model parameters under the uninformative 200, 500, and $800 \mathrm{mg}$ dose level study designs.

\begin{tabular}{|c|c|c|c|c|c|c|c|c|c|}
\hline \multirow[b]{2}{*}{ Parameter } & \multicolumn{3}{|c|}{$200 \mathrm{mg}$ dose level } & \multicolumn{3}{|c|}{$500 \mathrm{mg}$ dose level } & \multicolumn{3}{|c|}{$800 \mathrm{mg}$ dose level } \\
\hline & FO & FOCE-I & LAP-I & FO & FOCE-I & LAP-I & FO & FOCE-I & LAP-I \\
\hline $\mathrm{V}_{\max }$ & 10 & 7.4 & 5.5 & 59 & 32 & 12 & 121 & 67 & -2.8 \\
\hline $\mathrm{K}_{\mathrm{m}}$ & 48 & 5.0 & 6.5 & 268 & 63 & 86 & 1028 & 340 & 317 \\
\hline $\mathrm{CL}_{\mathrm{L}}$ & -16 & -3.0 & -4.3 & -20 & -9.8 & -6.4 & -23 & -12 & -4.8 \\
\hline $\mathrm{V}_{1}$ & -3.2 & -1.1 & -1.6 & -3.8 & -1.3 & -1.5 & -4.1 & -1.5 & -1.4 \\
\hline $\mathrm{V}_{2}$ & 31 & -1.3 & -4.1 & 25 & 0.33 & -2.3 & 24 & -1.3 & -1.9 \\
\hline Q & 21 & -0.10 & 1.3 & 20 & 1.9 & 2.5 & 21 & 2.7 & 2.2 \\
\hline $\mathrm{WGT} \sim \mathrm{V}_{\max }$ & -26 & -4.8 & 4.2 & -21 & 2.9 & 3.7 & -13 & 8.8 & -6.7 \\
\hline $\mathrm{WGT} \sim \mathrm{CL}_{\mathrm{L}}$ & 11 & -6.1 & -9.7 & -13 & -17 & -17 & -17 & -18 & -4.2 \\
\hline $\mathrm{WGT} \sim \mathrm{V}_{1}$ & -2.6 & -0.70 & -0.57 & -2.0 & -0.33 & -0.71 & -2.1 & -0.25 & -1.0 \\
\hline $\mathrm{WGT} \sim \mathrm{V}_{2}$ & 42 & 5.5 & 5.5 & 47 & 16 & 18 & 36 & 16 & 18 \\
\hline $\mathrm{V}_{\max } \operatorname{BSV}\left(\omega^{2}\right)$ & -57 & -13 & -14 & -74 & -29 & -7.7 & -89 & -61 & 61 \\
\hline $\mathrm{CL}_{\mathrm{L}} \mathrm{BSV}\left(\omega^{2}\right)$ & 33 & -7.6 & 1.8 & 2.4 & -1.4 & -2.3 & -2.8 & 1.4 & -6.9 \\
\hline $\mathrm{V}_{1} \operatorname{BSV}\left(\omega^{2}\right)$ & -4.2 & -6.6 & -5.0 & -5.5 & -6.9 & -6.2 & -6.4 & -7.2 & -5.9 \\
\hline $\mathrm{V}_{2} \operatorname{BSV}\left(\omega^{2}\right)$ & 240 & -98 & -83 & 94 & -100 & -94 & 16 & -100 & 2.17 \\
\hline Residual variance $\left(\sigma^{2}\right)$ & 11 & -2.6 & -2.3 & 7.3 & -2.5 & -2.5 & 6.6 & -2.7 & -2.3 \\
\hline
\end{tabular}

BSV $=$ between-subject variance; WGT $=$ weight. 
Table C-2. Precision (median absolute percent estimation error) in the population pharmacokinetic model parameters under the uninformative 200, 500, and $800 \mathrm{mg}$ dose level study designs.

\begin{tabular}{|c|c|c|c|c|c|c|c|c|c|}
\hline \multirow[b]{2}{*}{ Parameter } & \multicolumn{3}{|c|}{$200 \mathrm{mg}$ dose level } & \multicolumn{3}{|c|}{$500 \mathrm{mg}$ dose level } & \multicolumn{3}{|c|}{$800 \mathrm{mg}$ dose level } \\
\hline & FO & FOCE-I & LAP-I & FO & FOCE-I & LAP-I & FO & FOCE-I & LAP-I \\
\hline $\mathrm{V}_{\max }$ & 44 & 19 & 20 & 87 & 42 & 39 & 121 & 78 & 58 \\
\hline $\mathrm{K}_{\mathrm{m}}$ & 77 & 27 & 30 & 268 & 97 & 100 & 1028 & 340 & 317 \\
\hline $\mathrm{CL}_{\mathrm{L}}$ & 37 & 19 & 21 & 26 & 16 & 15 & 25 & 17 & 14 \\
\hline $\mathrm{V}_{1}$ & 4.1 & 3.0 & 3.3 & 4.4 & 3.2 & 3.3 & 4.6 & 3.2 & 3.2 \\
\hline $\mathrm{V}_{2}$ & 33 & 10 & 12 & 30 & 15 & 17 & 29 & 17 & 19 \\
\hline Q & 26 & 13 & 14 & 23 & 15 & 17 & 25 & 17 & 17 \\
\hline $\mathrm{WGT} \sim \mathrm{V}_{\max }$ & 94 & 60 & 61 & 107 & 89 & 90 & 125 & 121 & 108 \\
\hline $\mathrm{WGT} \sim \mathrm{CL}_{\mathrm{L}}$ & 136 & 77 & 82 & 102 & 79 & 73 & 105 & 81 & 70 \\
\hline $\mathrm{WGT} \sim \mathrm{V}_{1}$ & 27 & 22 & 23 & 27 & 24 & 24 & 28 & 23 & 24 \\
\hline $\mathrm{WGT} \sim \mathrm{V}_{2}$ & 136 & 68 & 73 & 126 & 98 & 101 & 115 & 105 & 113 \\
\hline $\mathrm{V}_{\max } \operatorname{BSV}\left(\omega^{2}\right)$ & 73 & 33 & 33 & 100 & 68 & 64 & 100 & 100 & 100 \\
\hline $\mathrm{CL}_{\mathrm{L}} \operatorname{BSV}\left(\omega^{2}\right)$ & 100 & 41 & 39 & 61 & 32 & 30 & 61 & 31 & 27 \\
\hline $\mathrm{V}_{1} \operatorname{BSV}\left(\omega^{2}\right)$ & 21 & 18 & 19 & 21 & 19 & 20 & 22 & 20 & 20 \\
\hline $\mathrm{V}_{2} \operatorname{BSV}\left(\omega^{2}\right)$ & 240 & 100 & 100 & 100 & 100 & 100 & 100 & 100 & 100 \\
\hline Residual variance $\left(\sigma^{2}\right)$ & 12 & 5.3 & 5.7 & 8.6 & 5.2 & 5.6 & 8.1 & 5.3 & 5.5 \\
\hline
\end{tabular}

BSV $=$ between-subject variance; WGT $=$ weight. 
Figure C-1. Markov Chain Monte Carlo chain histories (first set of plots), probability densities of the posterior distributions (second set of plots), and Gelman-Rubin-Brooks plots (third set of plots) for a representative estimation run using Bayesian informative priors under the informative study design. 


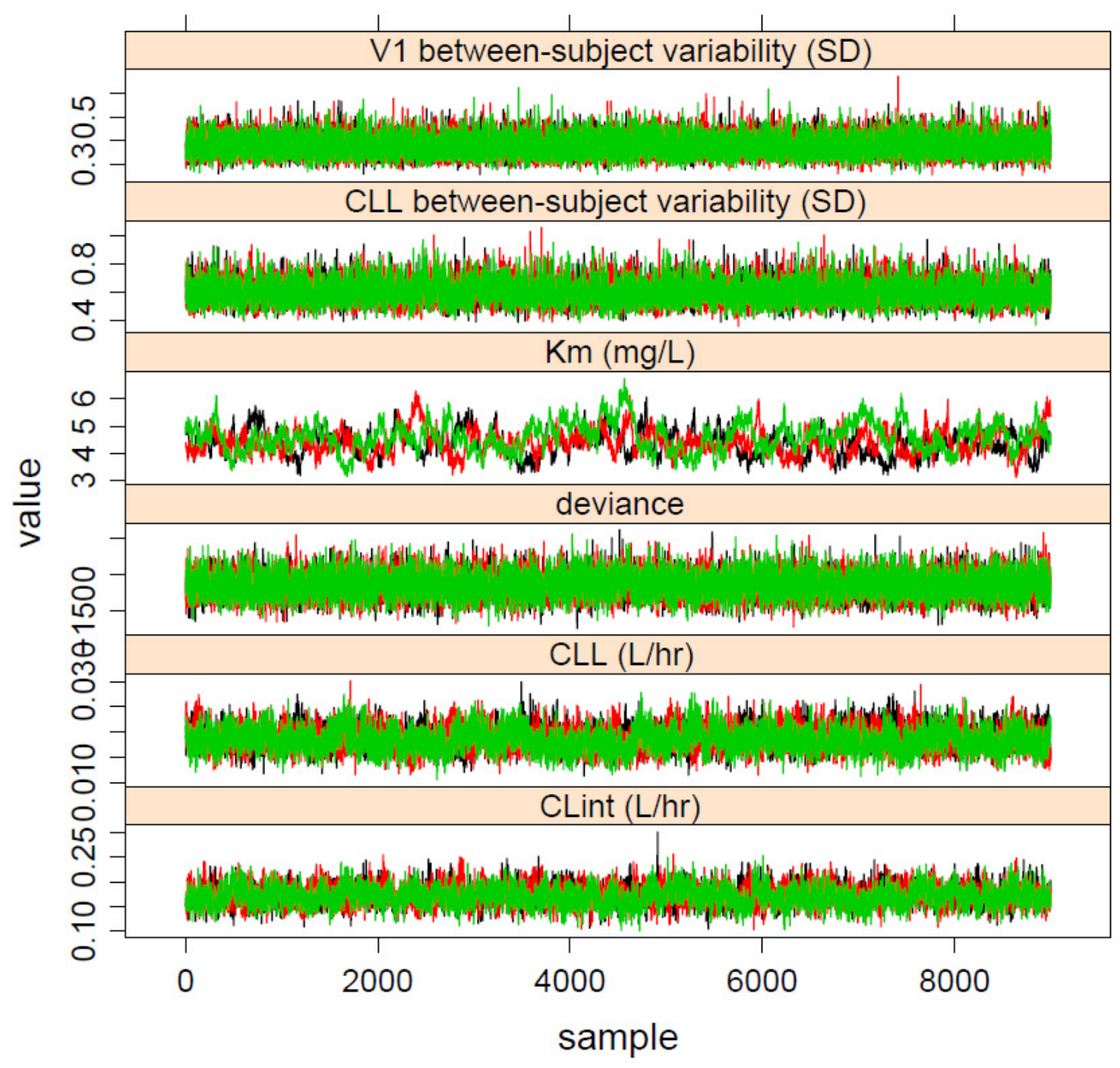




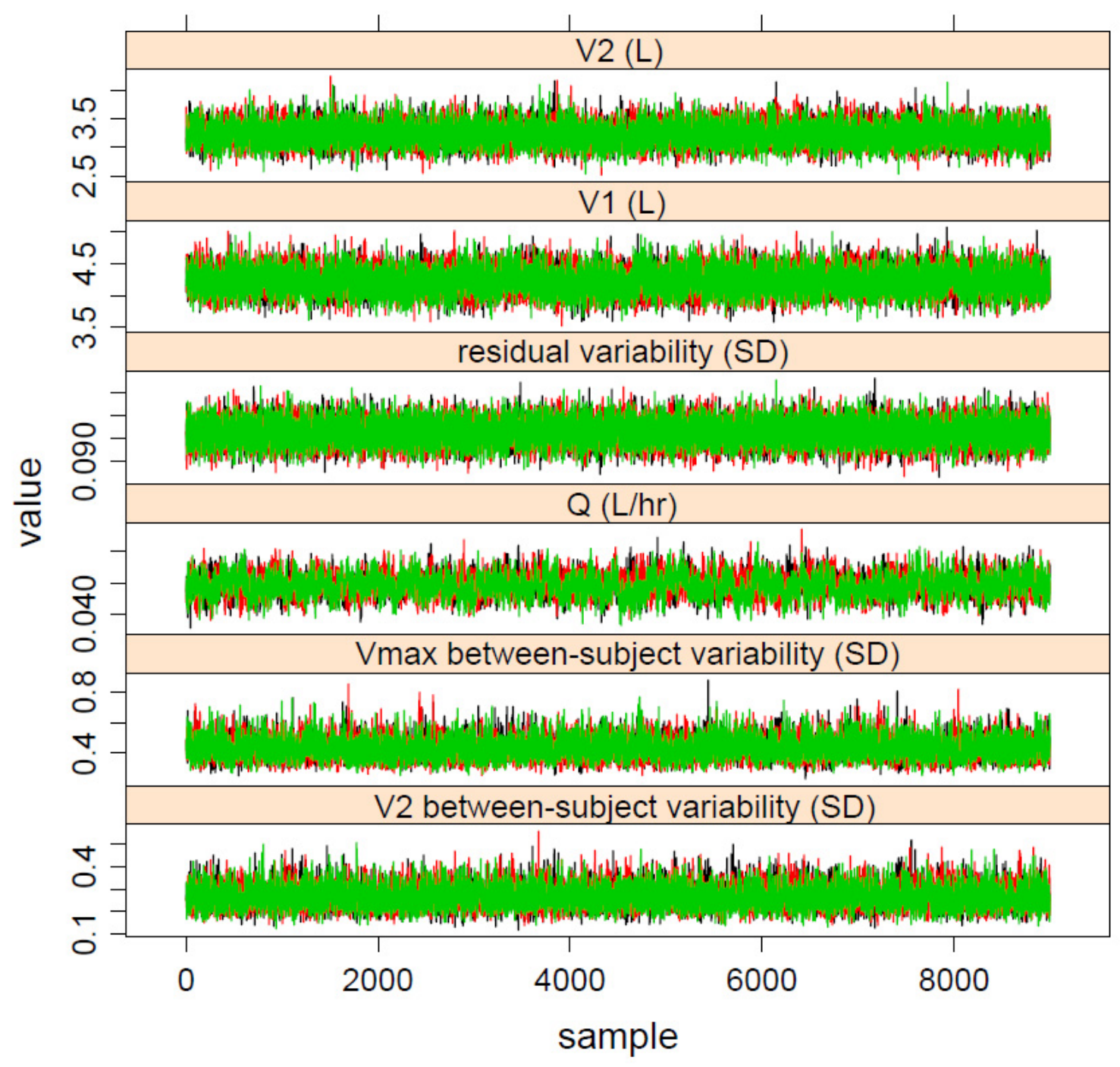

Figure C-1 continued. 


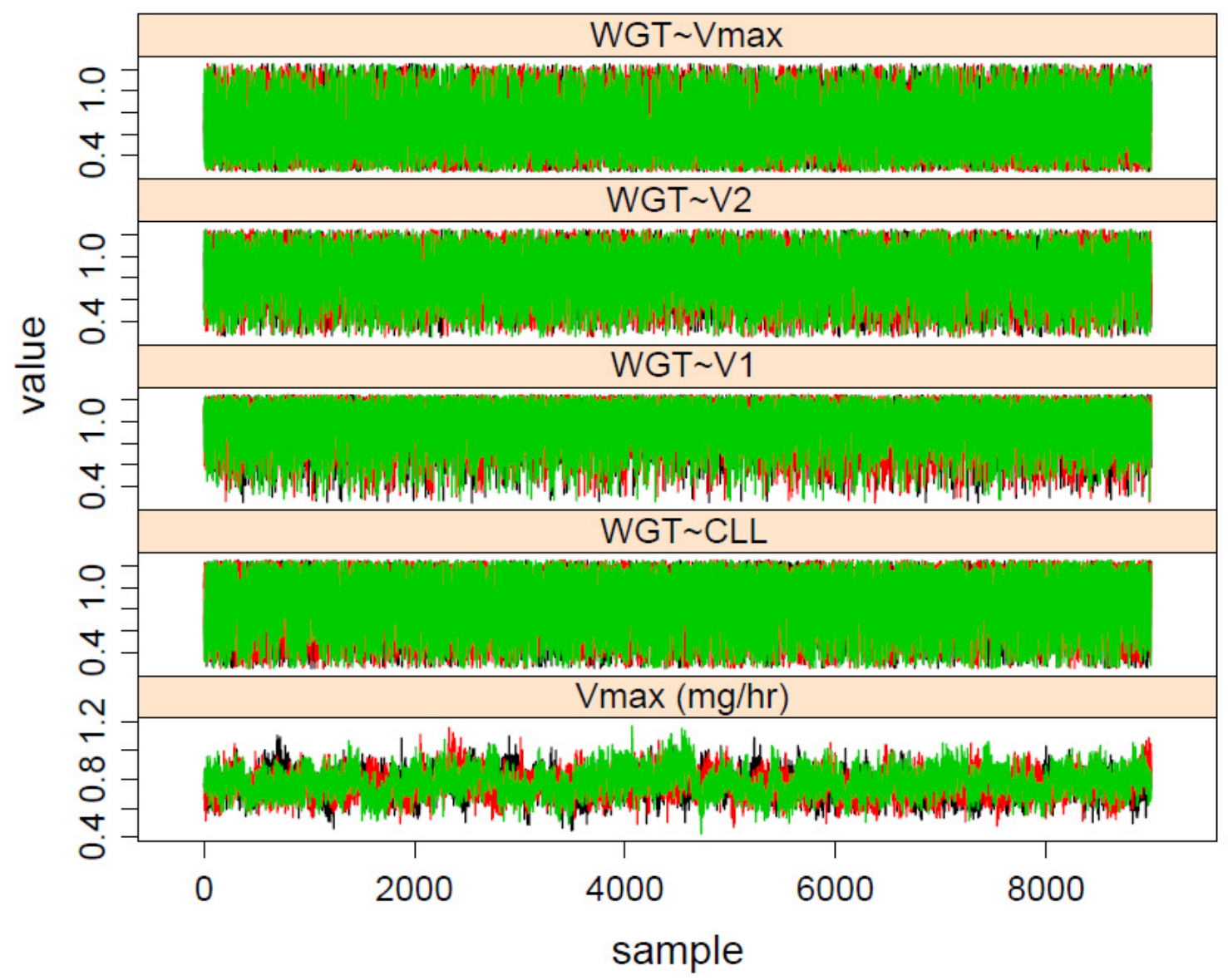

Figure C-1 continued. 


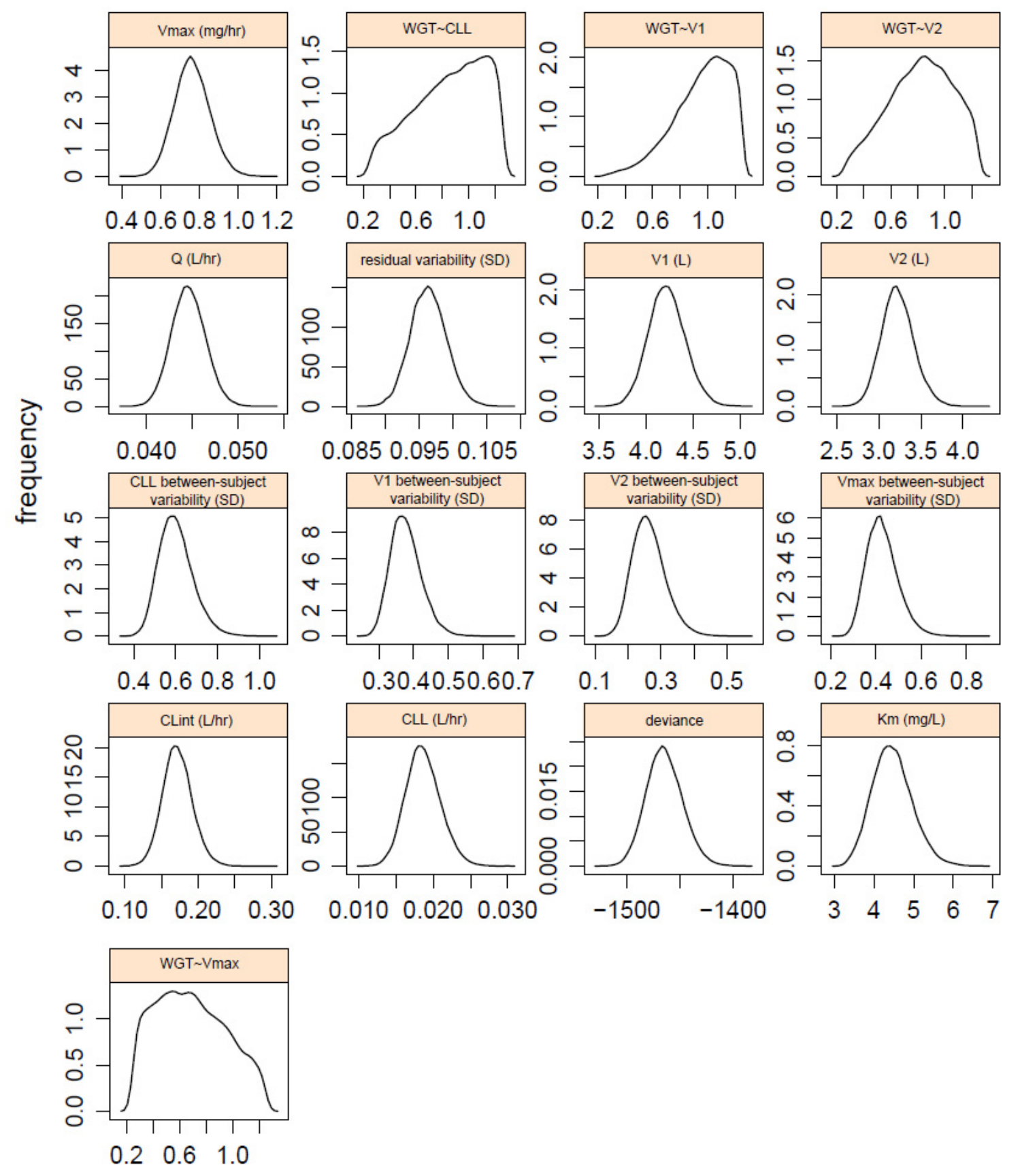

value

Figure C-1 continued. 


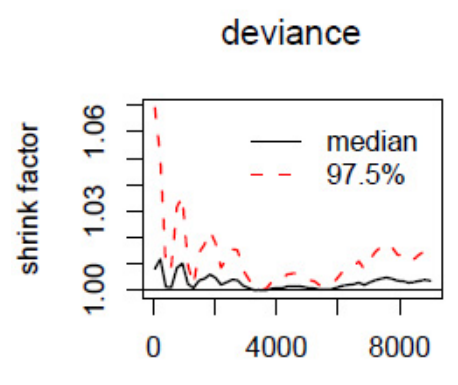

last iteration in chain

CLint (L/hr)

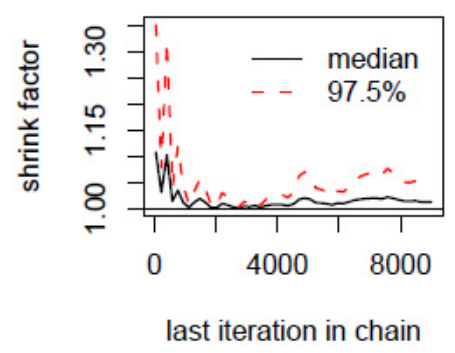

$\mathrm{Km}(\mathrm{mg} / \mathrm{L})$

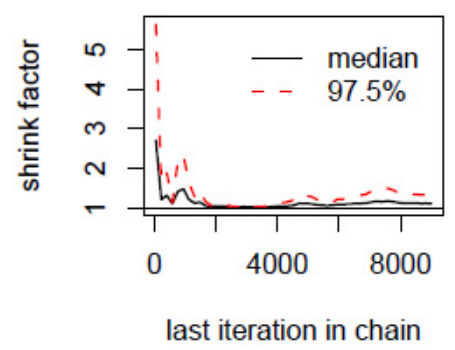

$\operatorname{Vmax}(\mathrm{mg} / \mathrm{hr})$

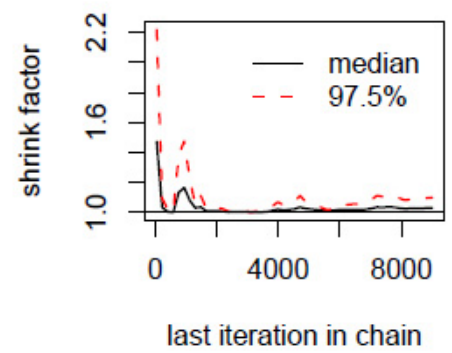

V1 (L)

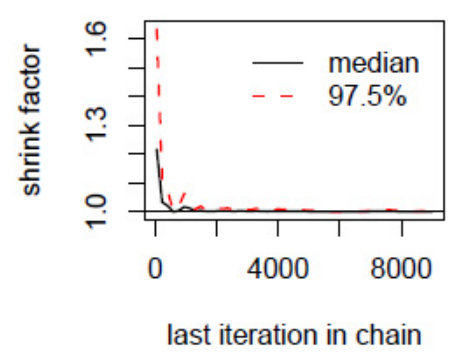

$Q(L / h r)$

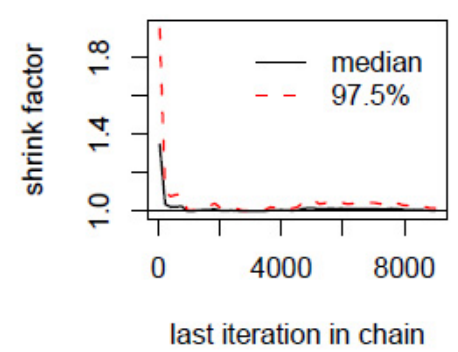

CLL (L/hr)

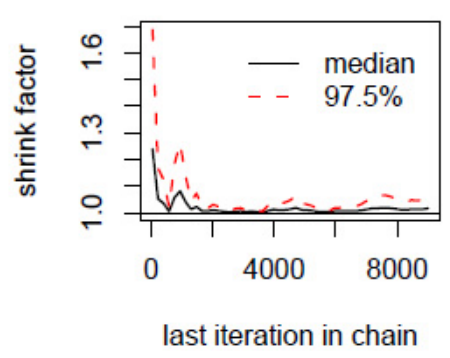

V2 (L)

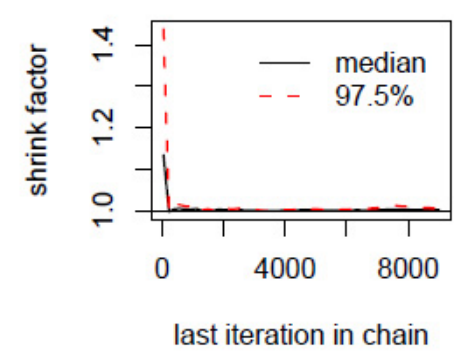

WGT Vmax

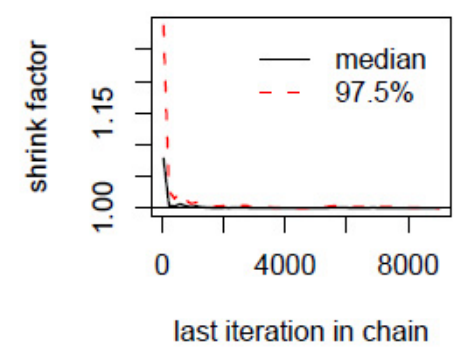

Figure C-1 continued. 
WGT CLL

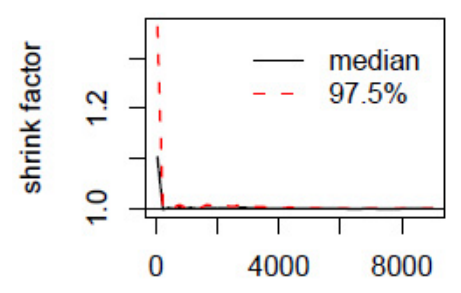

last iteration in chain

Vmax between-subject variability $(\mathrm{SD})$

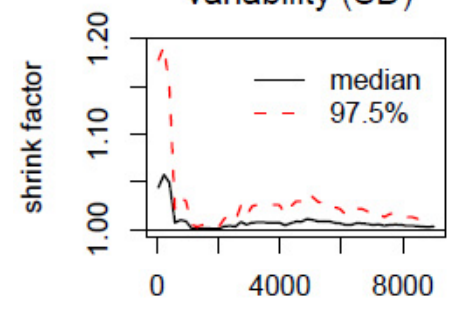

last iteration in chain

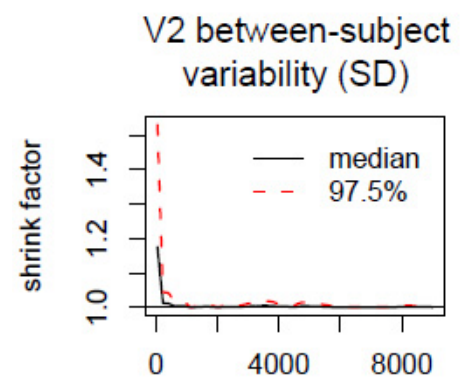

last iteration in chain
WGT V1

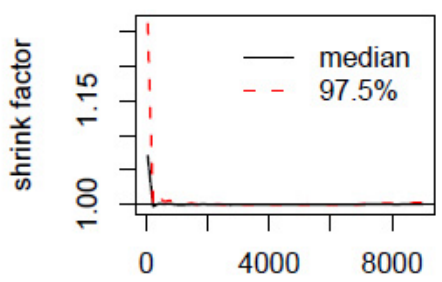

last iteration in chain

CLL between-subject variability (SD)

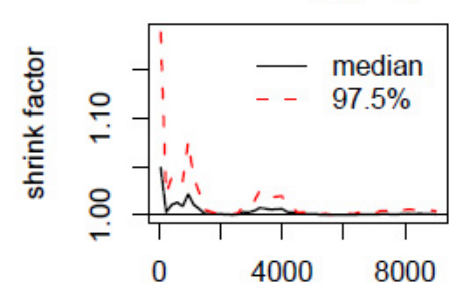

last iteration in chain

residual variability (SD)

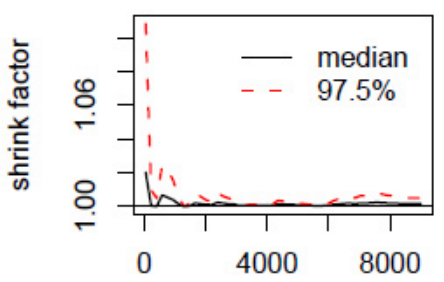

last iteration in chain
WGT V2

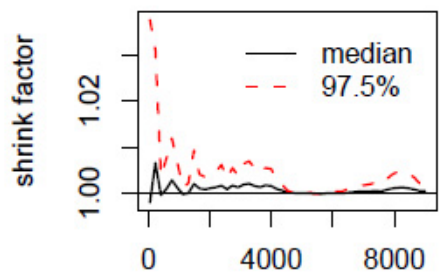

last iteration in chain

V1 between-subject variability (SD)

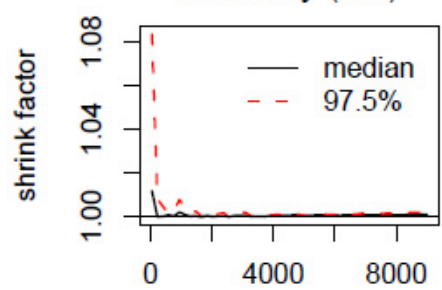

last iteration in chain

Figure C-1 continued. 
Figure C-2. Markov Chain Monte Carlo chain histories (first set of plots), probability densities of the posterior distributions (second set of plots), and Gelman-Rubin-Brooks plots (third set of plots) for a representative estimation run using Bayesian uninformative priors under the informative study design. 


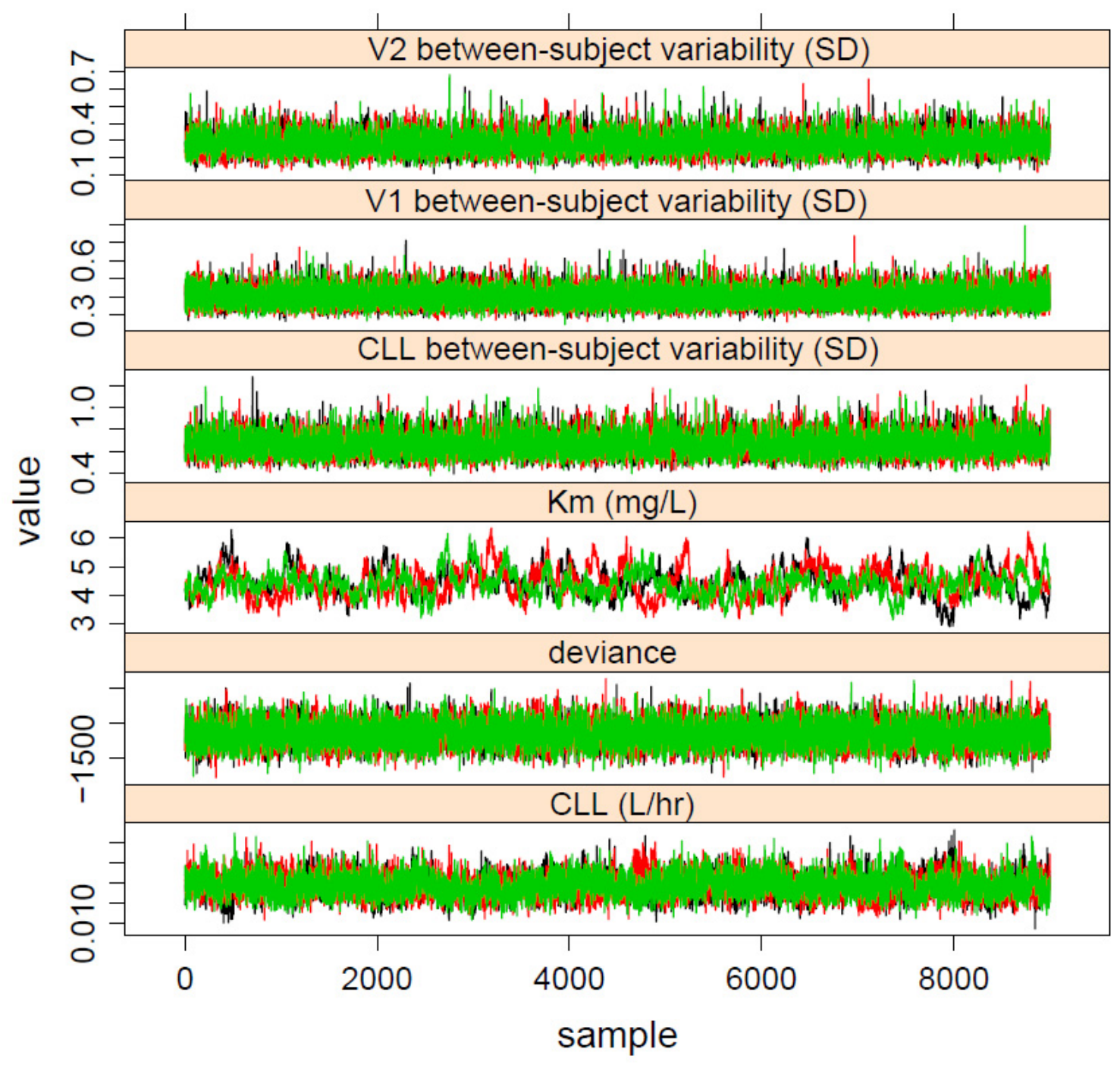




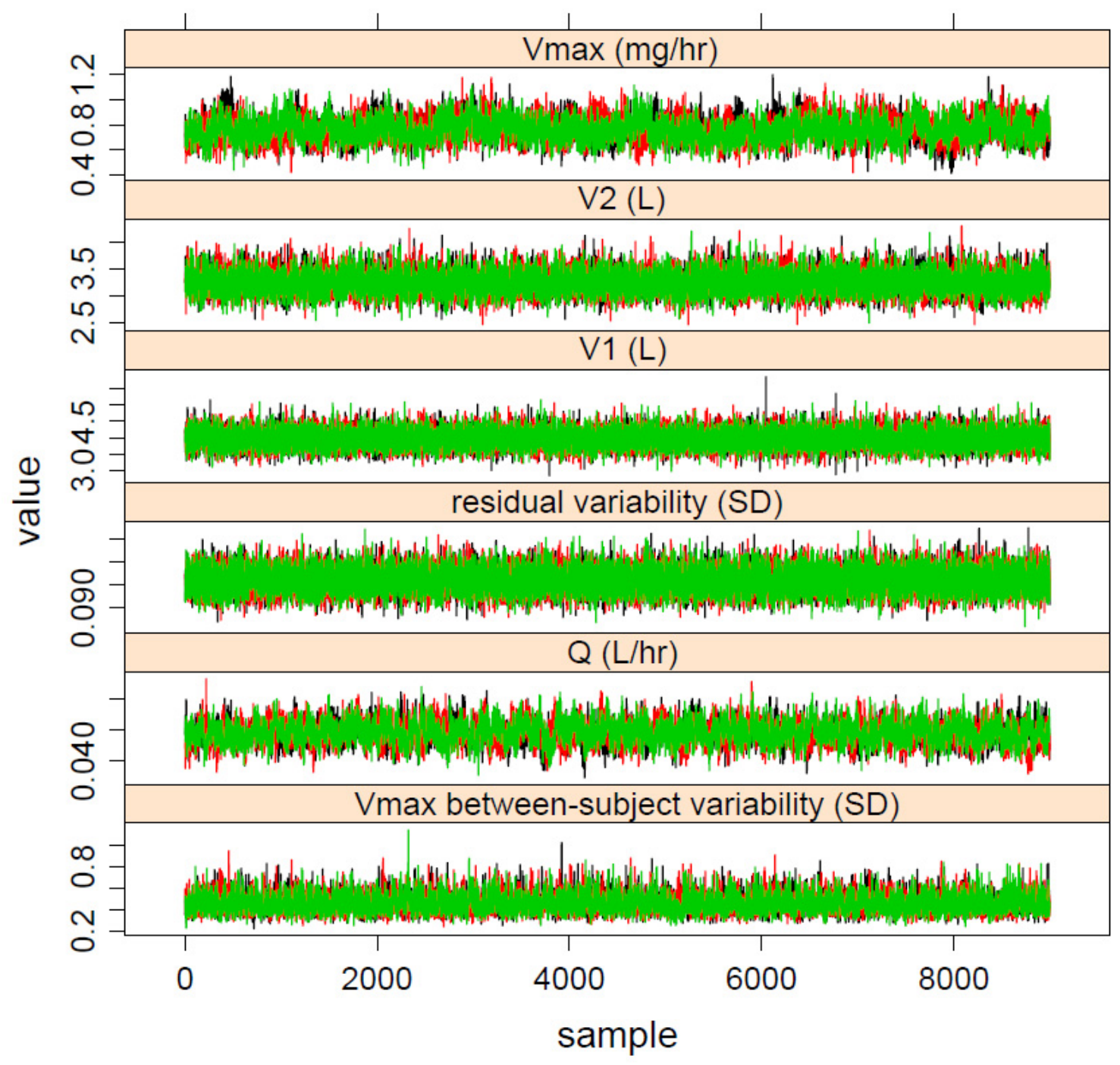

Figure C-2 continued. 


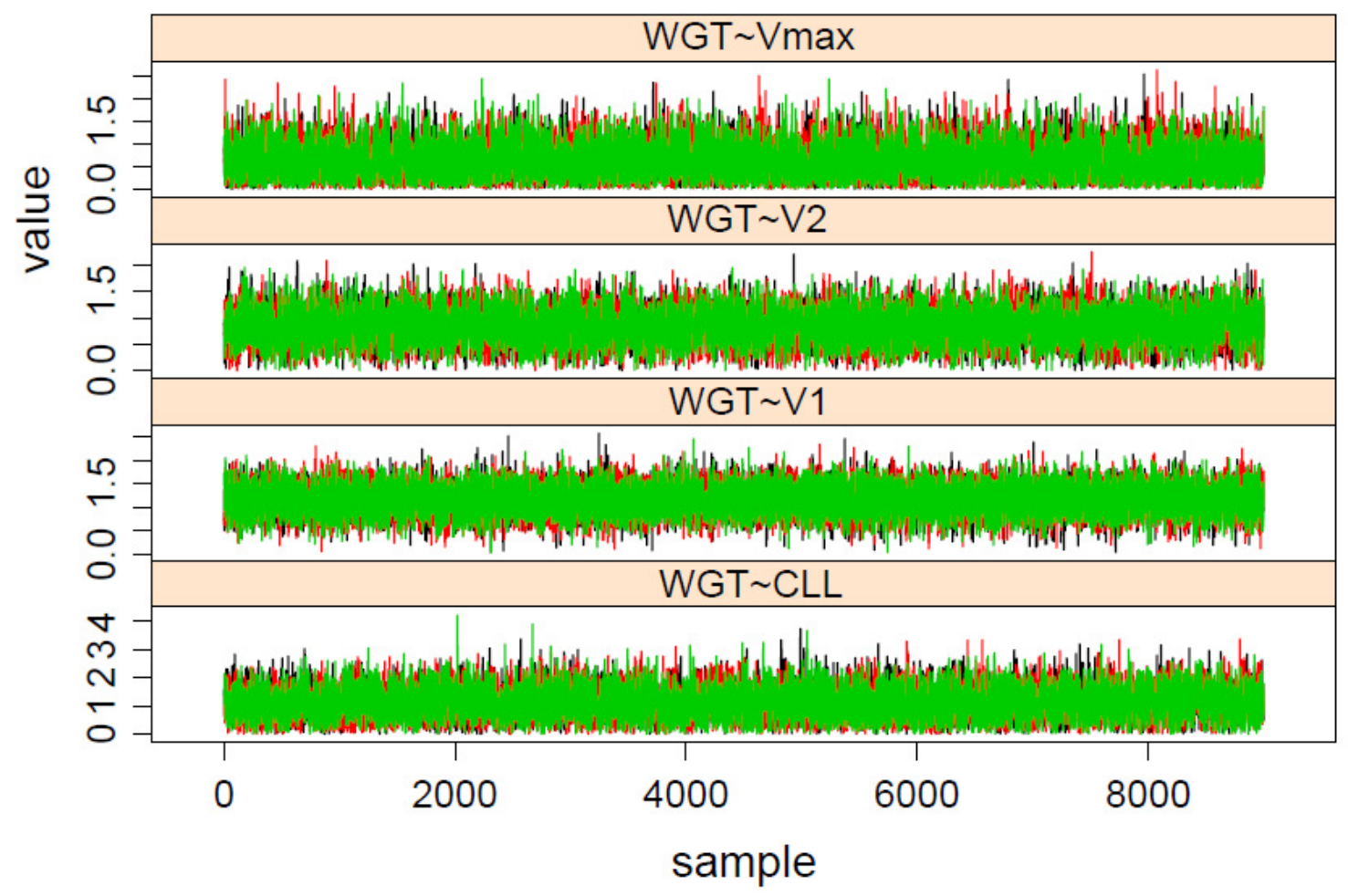

Figure C-2 continued. 

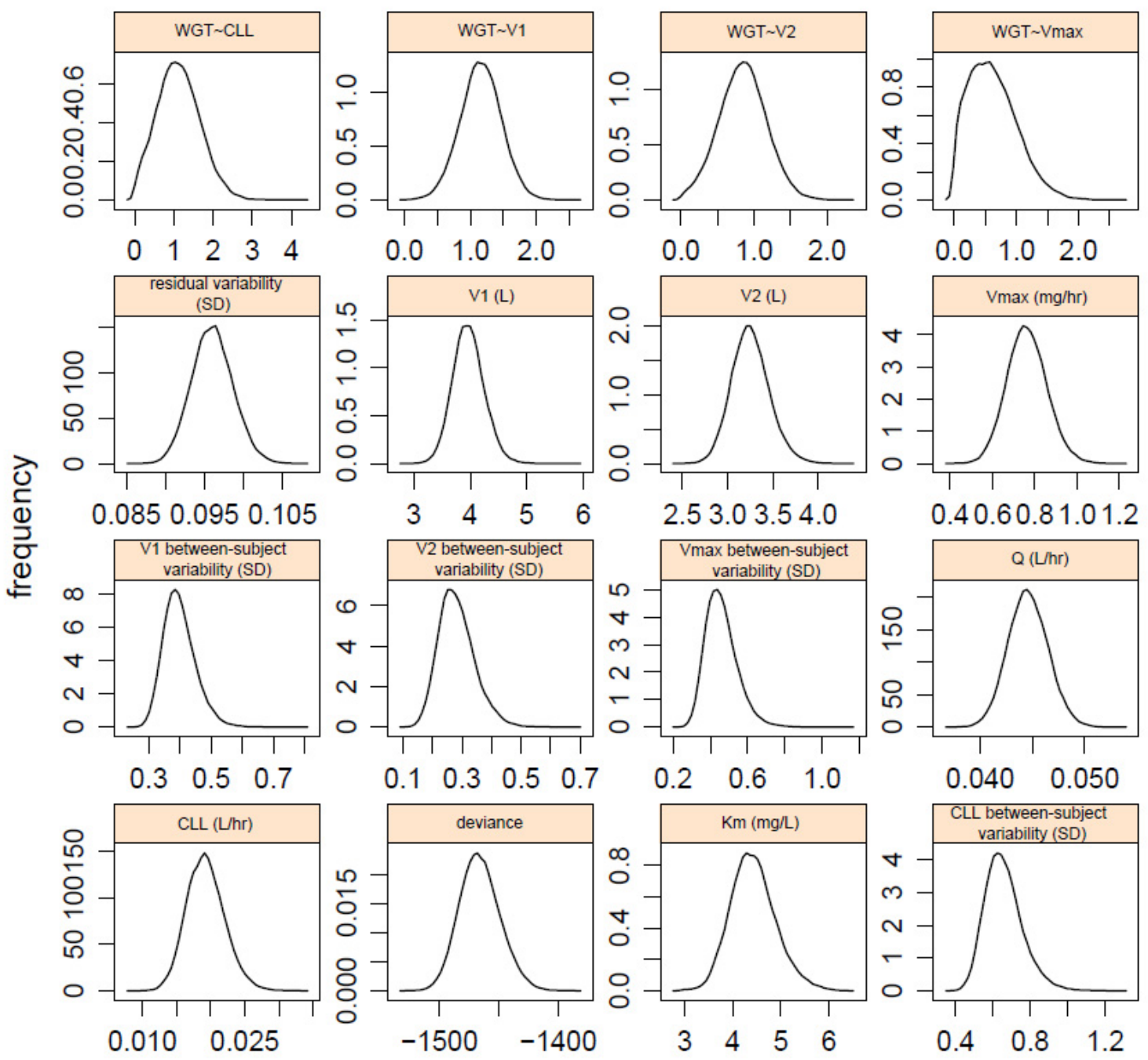

value

Figure C-2 continued. 


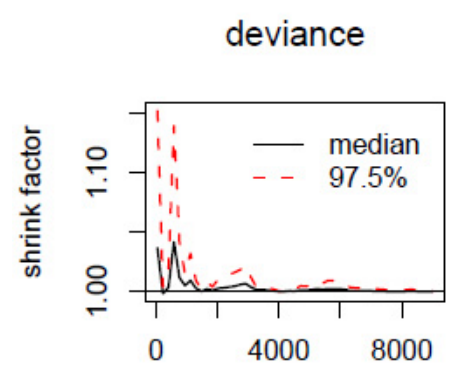

last iteration in chain

V1 (L)

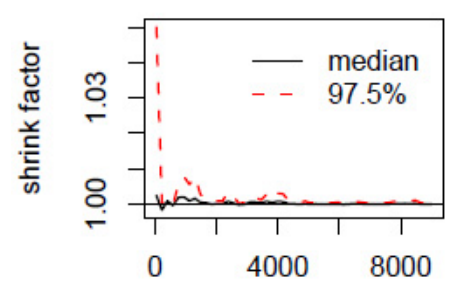

last iteration in chain

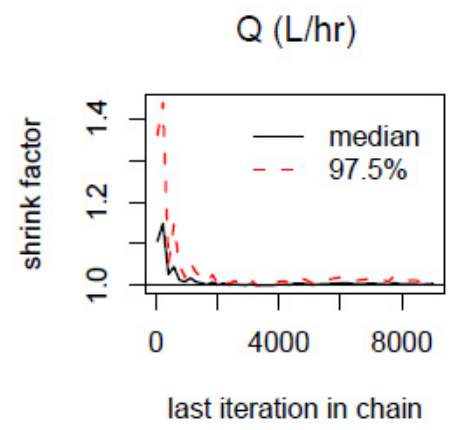

$\operatorname{Vmax}(\mathrm{mg} / \mathrm{hr})$

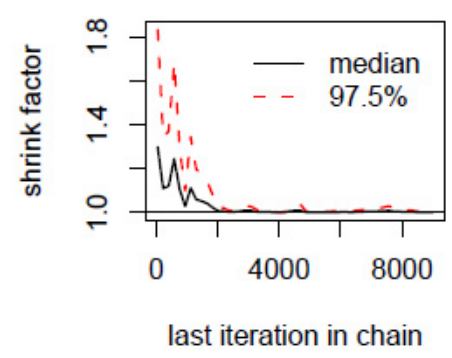

V2 (L)
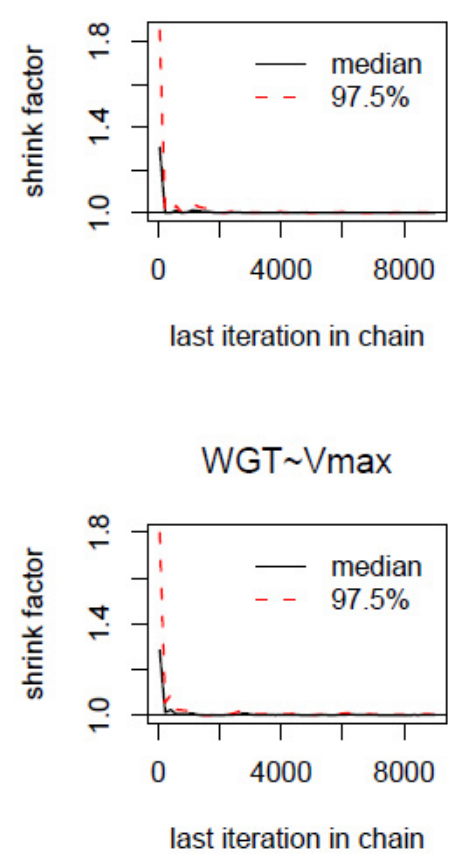

CLL (L/hr)

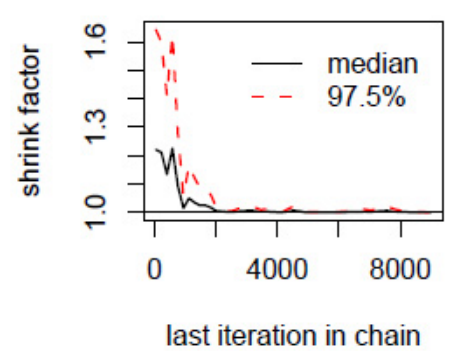

$\mathrm{Km}$ (mg/L)
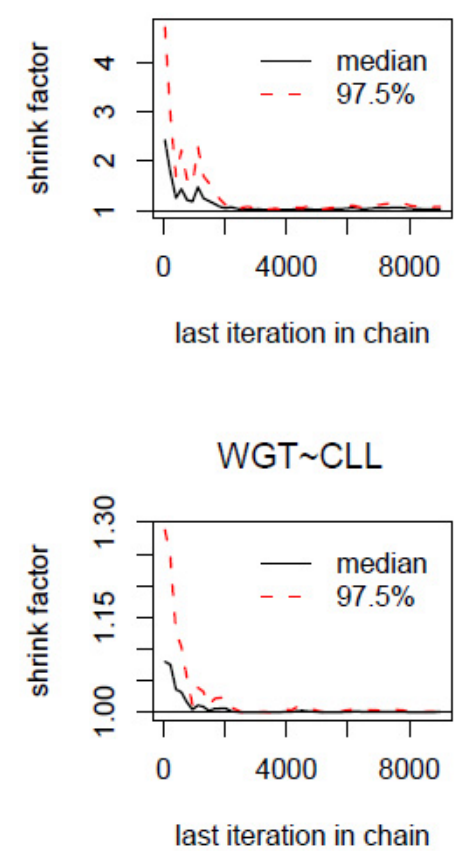

Figure C-2 continued. 
WGT V1

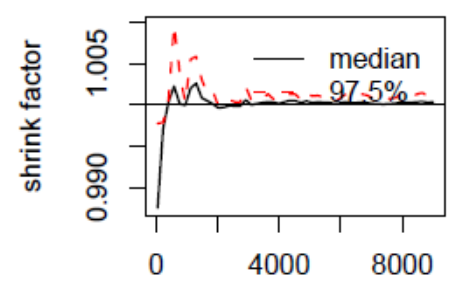

last iteration in chain

\section{CLL between-subject} variability $(\mathrm{SD})$

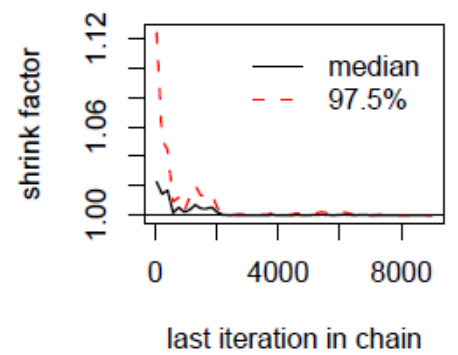

residual variability (SD)

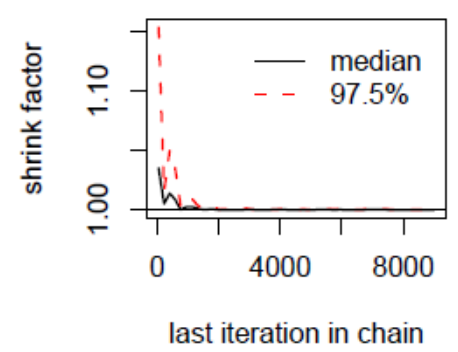

WGT V2

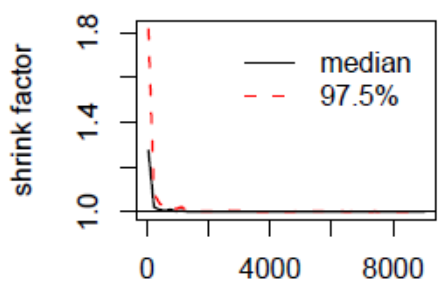

last iteration in chain

V1 between-subject variability $(S D)$

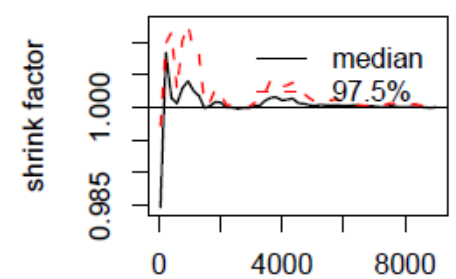

last iteration in chain
Vmax between-subject variability (SD)

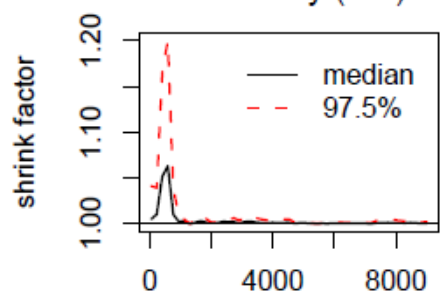

last iteration in chain

\section{V2 between-subject} variability (SD)

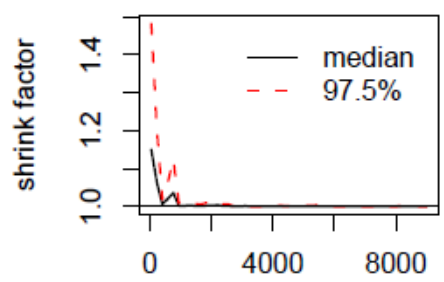

last iteration in chain

Figure C-2 continued. 
Figure C-3. Markov Chain Monte Carlo chain histories (first set of plots), probability densities of the posterior distributions (second set of plots), and Gelman-Rubin-Brooks plots (third set of plots) for a representative estimation run using Bayesian informative priors under the uninformative $600 \mathrm{mg}$ study design. 


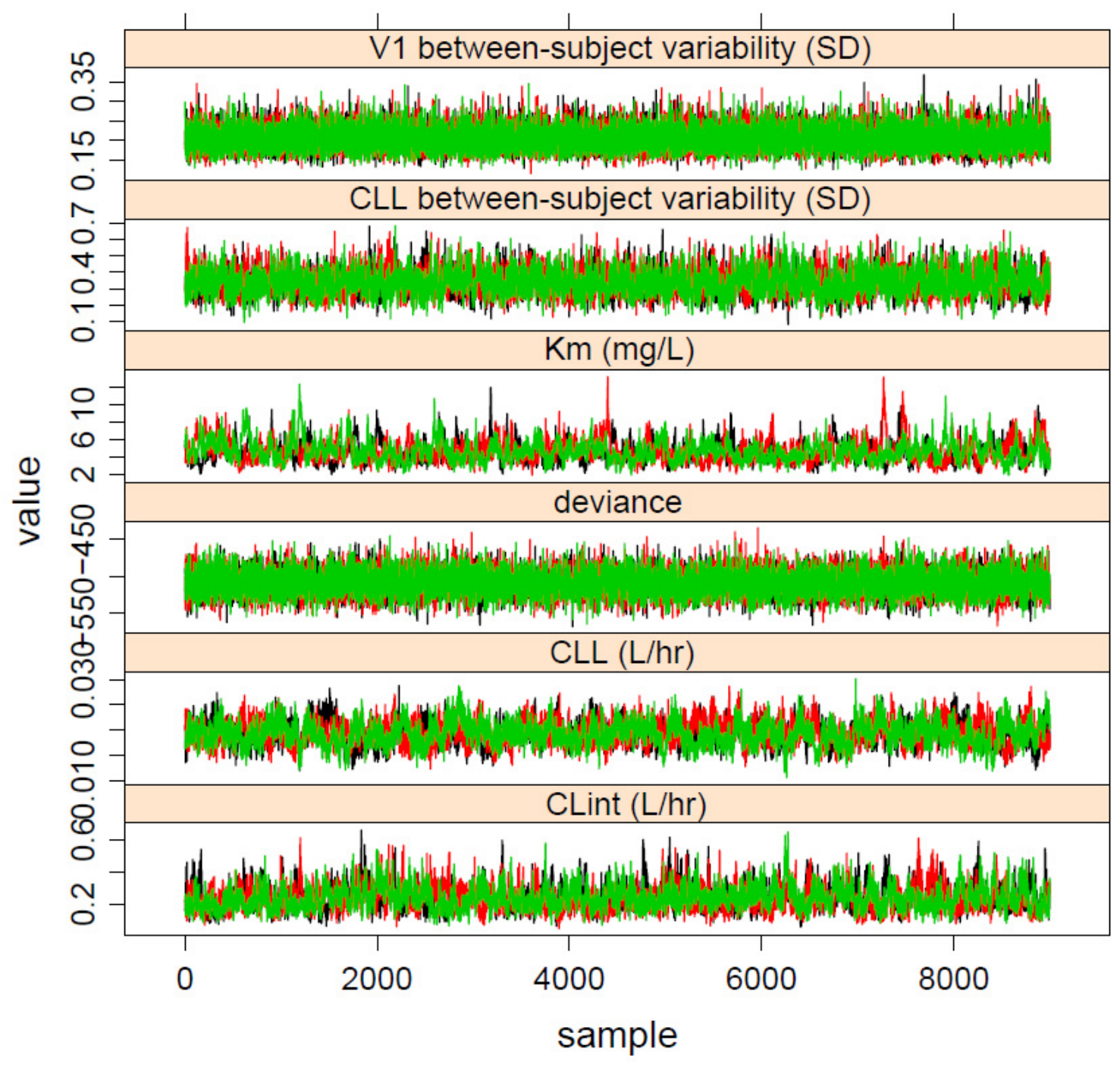




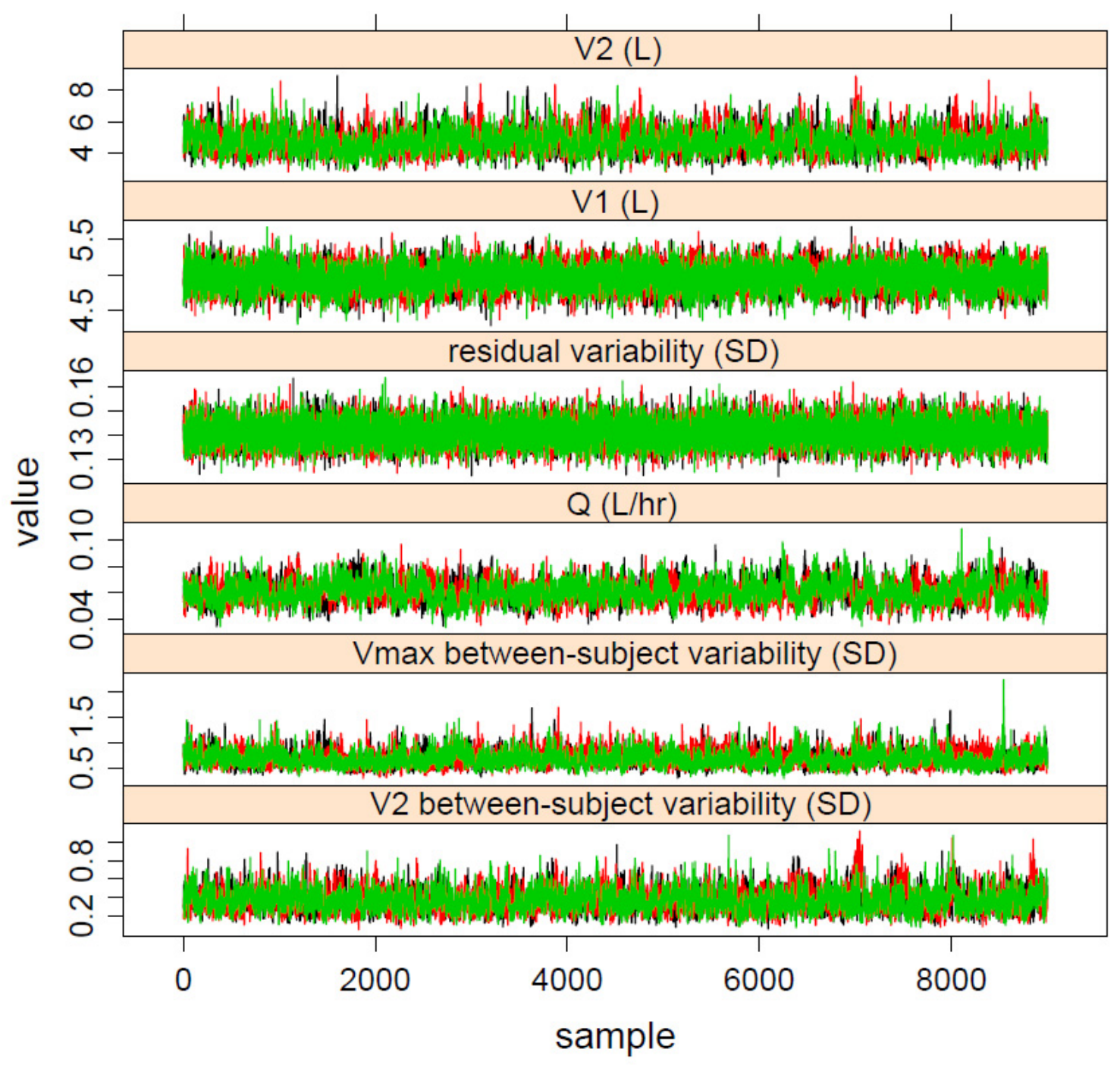

Figure C-3 continued. 


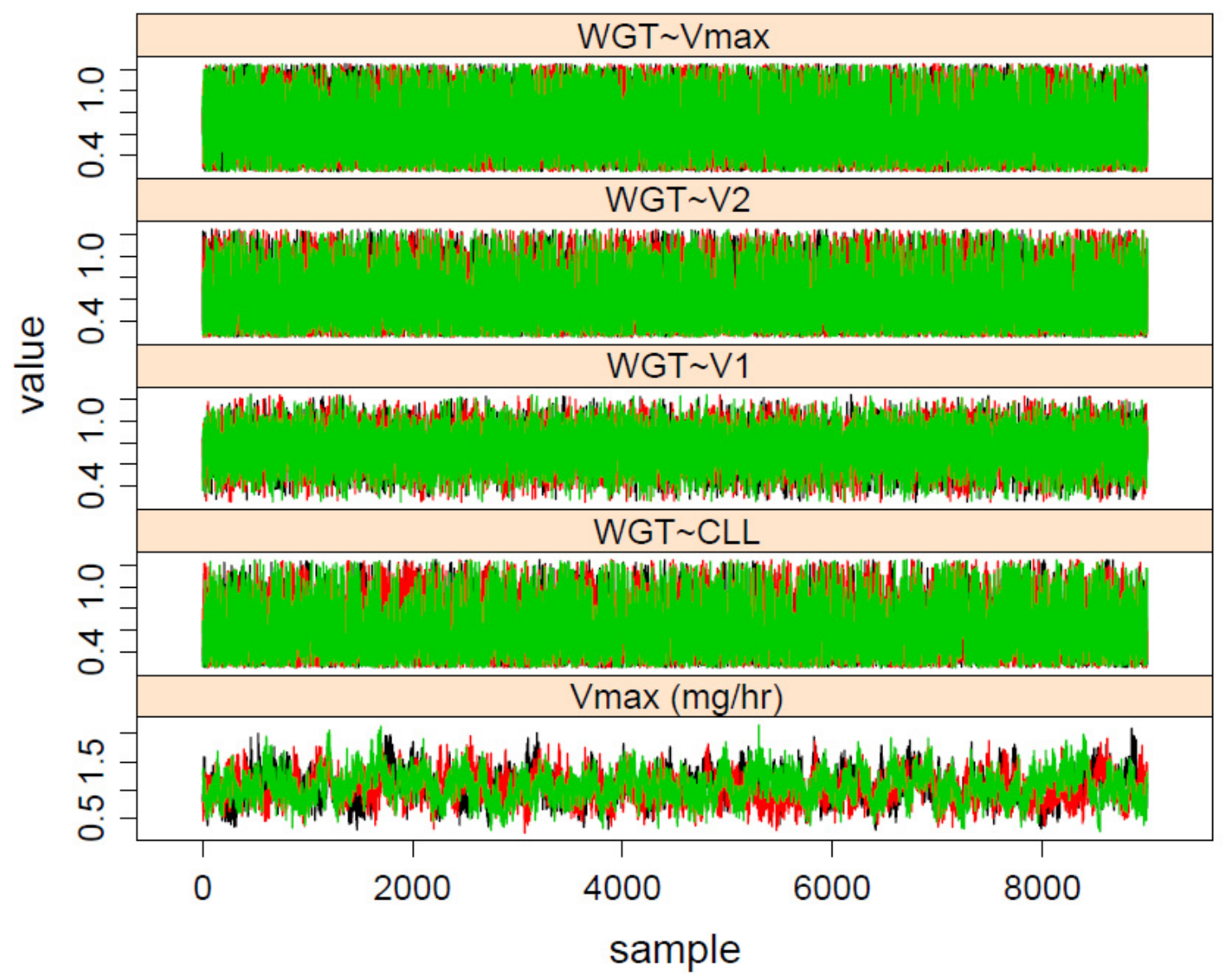

Figure C-3 continued. 


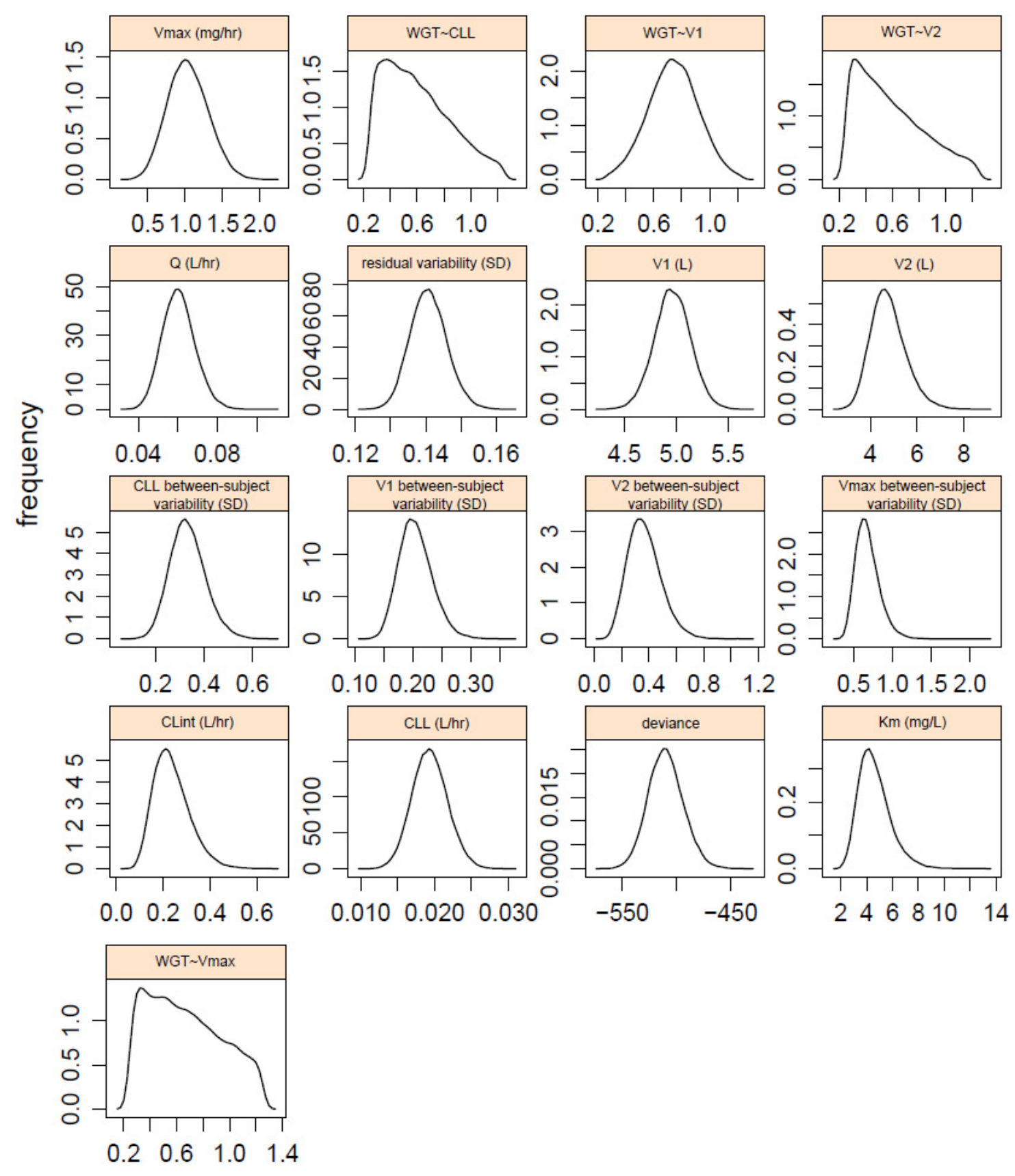

value

Figure C-3 continued. 


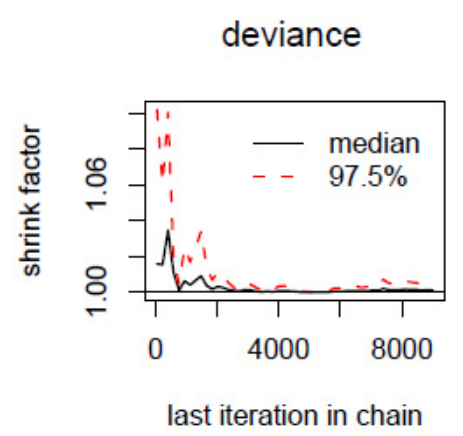

CLint (L/hr)

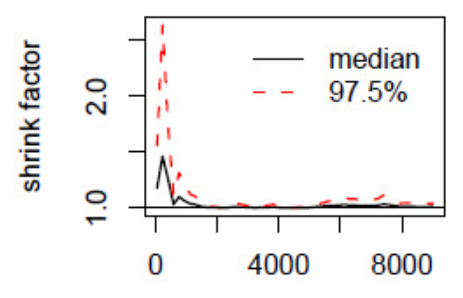

last iteration in chain

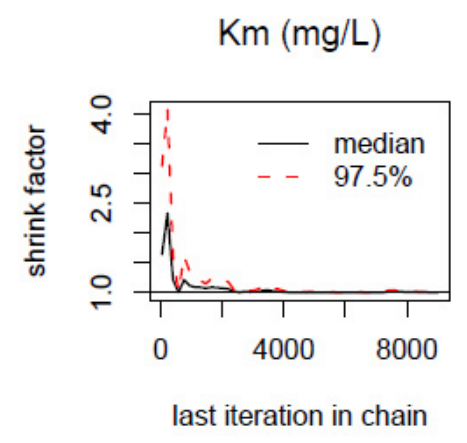

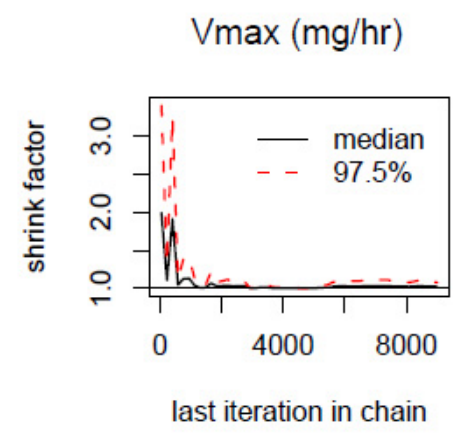

V1 (L)
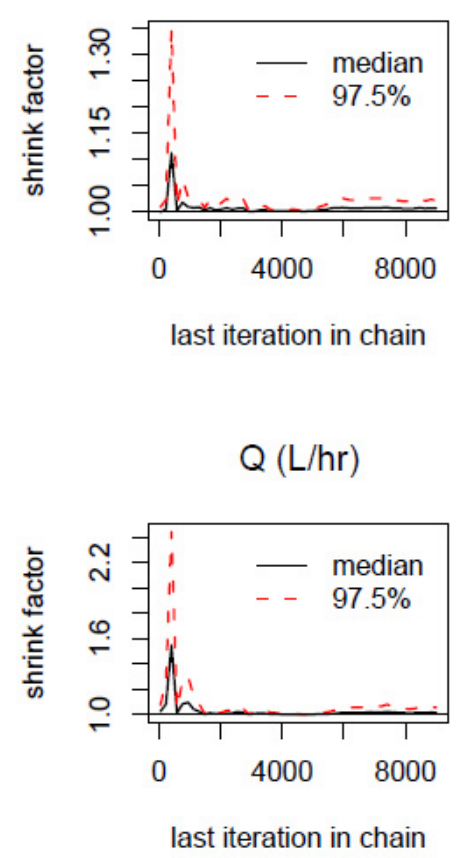

CLL (L/hr)

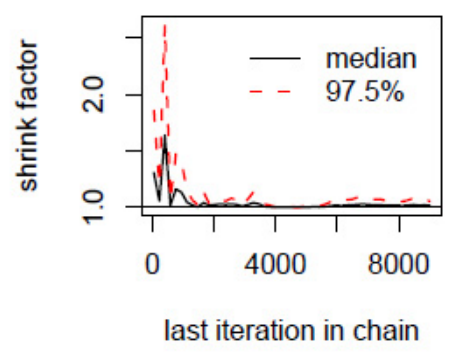

V2 (L)
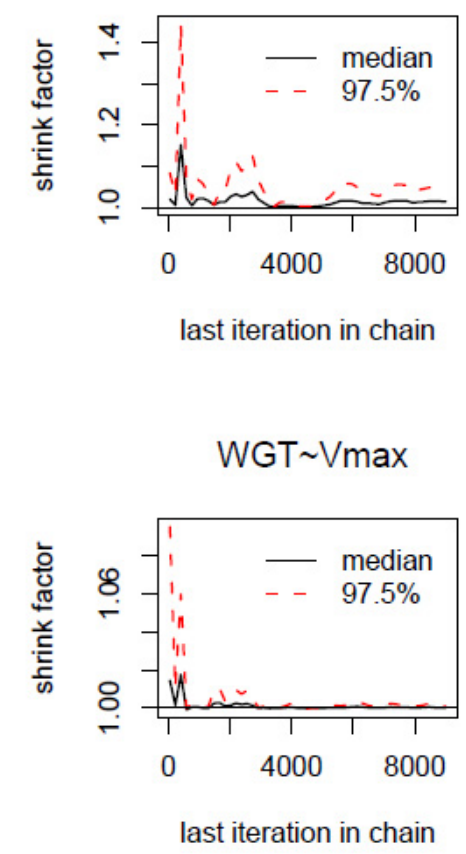

Figure C-3 continued. 


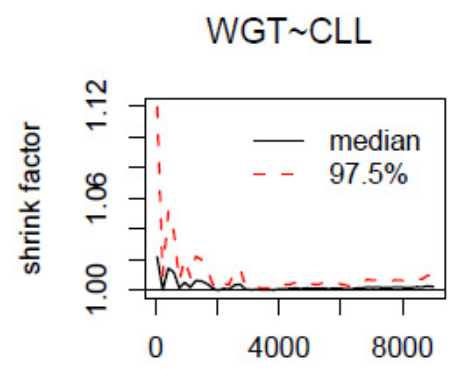

last iteration in chain

Vmax between-subject variability (SD)

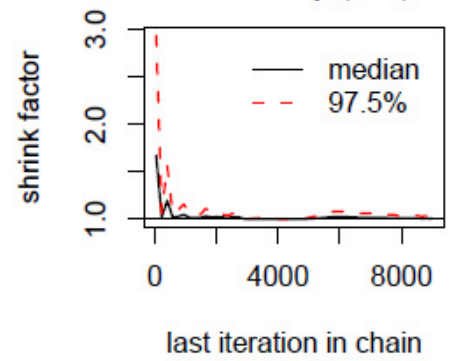

V2 between-subject
variability $(S D)$

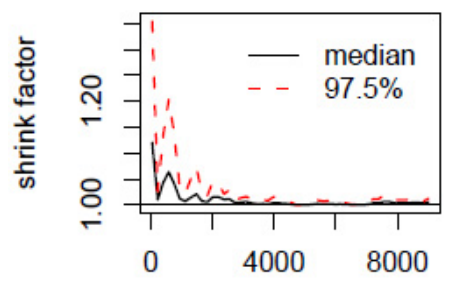

last iteration in chain
WGT V1

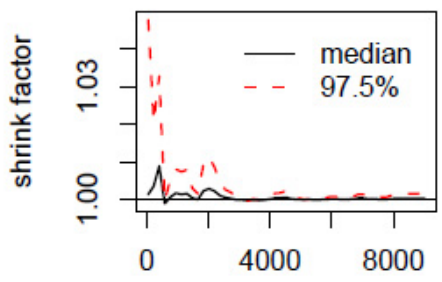

last iteration in chain

\section{CLL between-subject} variability (SD)

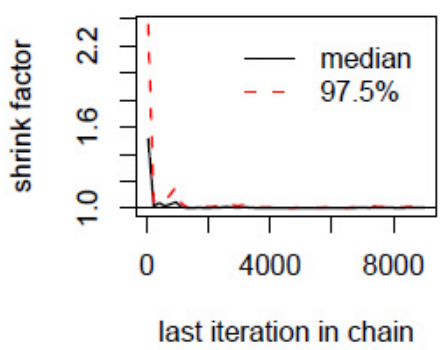

residual variability (SD)

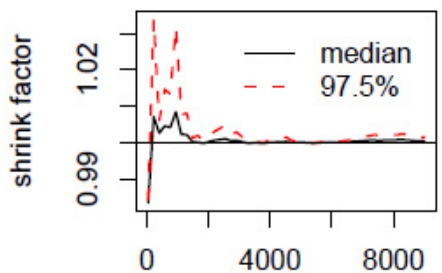

last iteration in chain

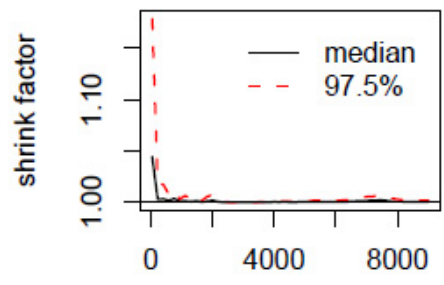

last iteration in chain

V1 between-subject variability (SD)

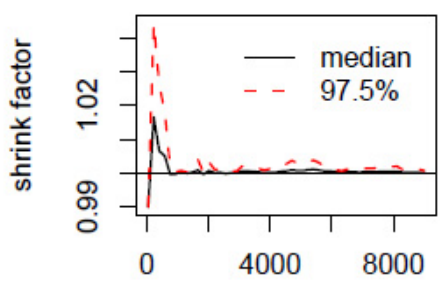

last iteration in chain

Figure C-3 continued. 


\section{VITA}

Nathanael Le Dirks was born in Fort Dodge, Iowa, to Randy and Rebecca Dirks on March 30, 1980. He enrolled at the University of Northern Iowa (Cedar Falls, Iowa) in 1997 and graduated in 2001 with a Bachelor of Arts in Biology: Biomedical and with Summa Cum Laude honors (cumulative GPA 4.0). While at UNI, he received a number of other accolades including the following: Purple and Old Gold Award for meritorious scholarship in biology, Merchant Scholarship, Clifford McCollum Scholarship, C.W. Lantz Scholarship, Outstanding New Biology Major Award, and was named to the Dean's List each semester. In 2001, he enrolled in the joint Doctor of Pharmacy and Doctor of Philosophy (Pharm.D./Ph.D.) program at the University of Tennessee Health Science Center in Memphis. In 2005, he received his Pharm.D., and graduated with Highest Honors (cumulative GPA 4.0) and was Valedictorian of his pharmacy class. During pharmacy school he received a number of other accolades including the following: Lilly Achievement Award, Medicinal Chemistry Award, Seldon D. Feurt Scholar (Pharm.D./Ph.D. scholarship), Seldon D. Feurt Memorial Scholarship, Plough Scholarship, and was inducted into the Rho Chi Pharmacy Honor Society and the UTHSC Imhotep Society. Following pharmacy school, he continued pursuing his Ph.D. under the direction of Dr. Bernd Meibohm. During graduate school he published five peer-reviewed articles, presented at a number of scientific meetings, maintained a cumulative GPA of 4.0, and received two American Foundation for Pharmaceutical Education (AFPE) Pre-Doctoral Fellowships in the Pharmaceutical Sciences. He received his Ph.D. with a major in Pharmaceutical Sciences in 2010, and following graduation he joined Metrum Research Group (Tariffville, Connecticut) as a research scientist. 